Supporting Information for

\title{
Silylpalladium Cations Enable the Cleavage of Nitrile C-CN Bonds
}

Andreas L. Wierschen, ${ }^{\dagger}$ Jared Lowe, ${ }^{\dagger, \$}$ Neyen Romano, ${ }^{\dagger,}$ Stephen J. Lee, ${ }^{\ddagger}$ and Michel R. Gagnét,

${ }^{\dagger}$ Caudill Laboratories, Department of Chemistry, The University of North Carolina at Chapel Hill, Chapel Hill, North Carolina 27599-3290, United States

‡U.S. Army Research Office, P.O. Box 12211, Research Triangle Park, North Carolina 27709, United States

\section{Table of Contents}

GENERAL METHODS

REACTION PROCEDURES AND COMPLEX SYNTHESES

FIGURE S1:

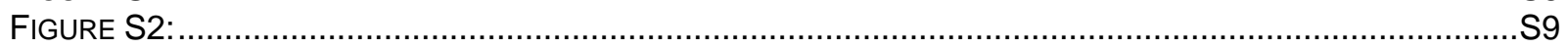

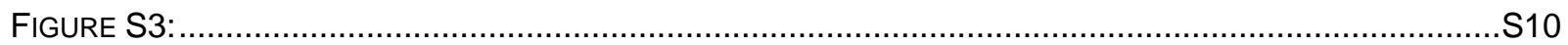

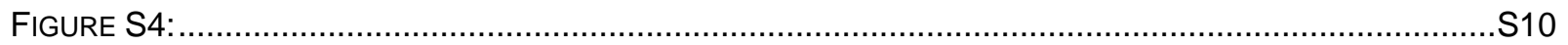

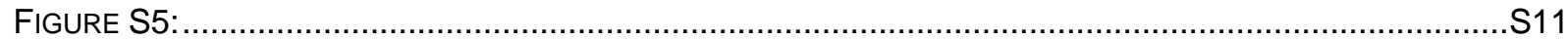

FIGURE S6:

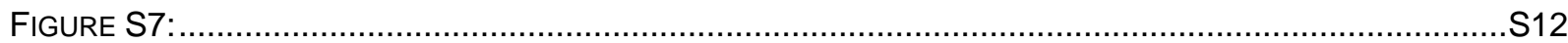

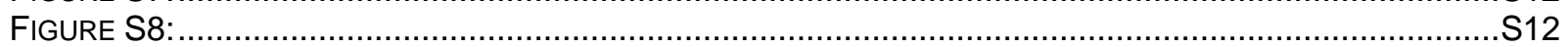

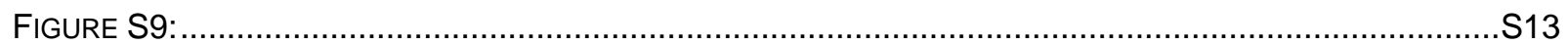

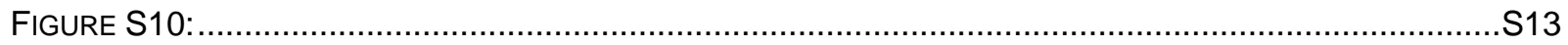

FIGURE S11:

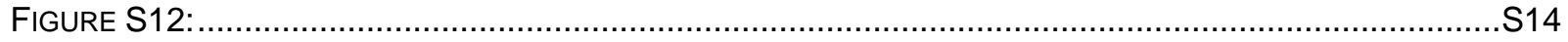

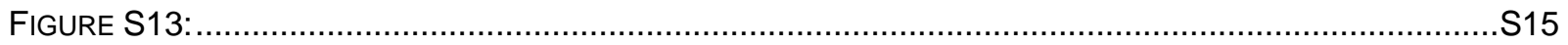

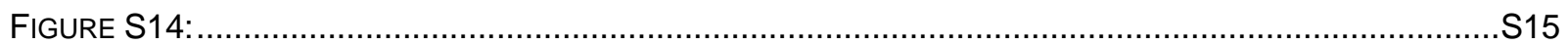

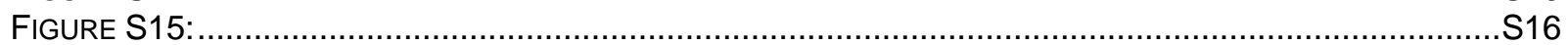

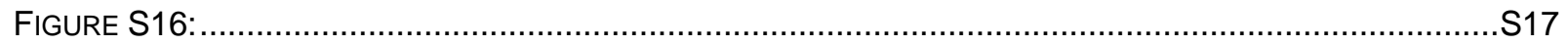

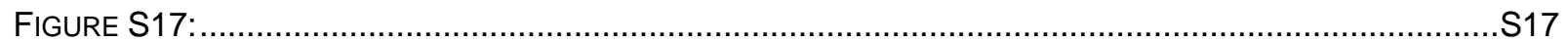

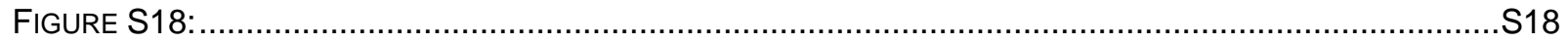

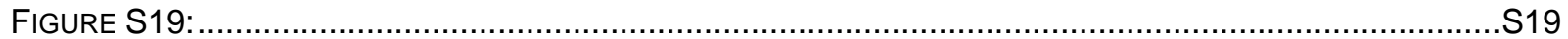

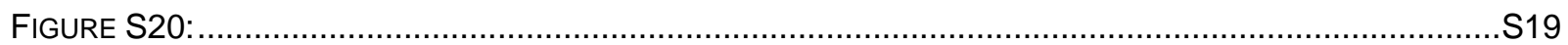

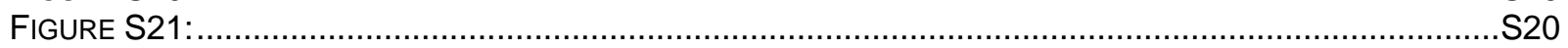

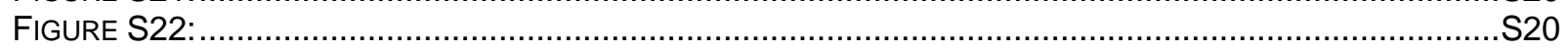

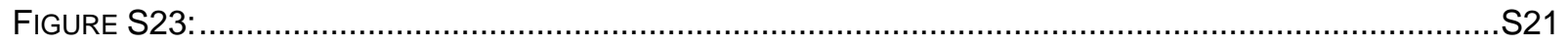

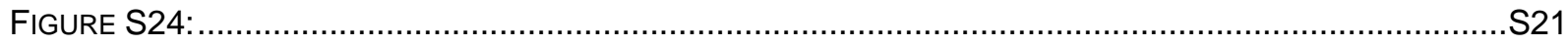

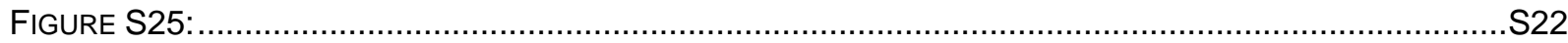

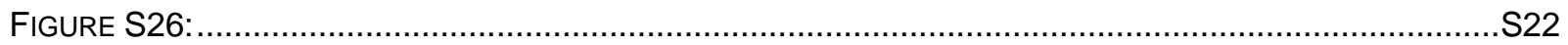

FIGURE S27:

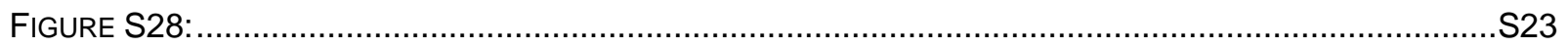

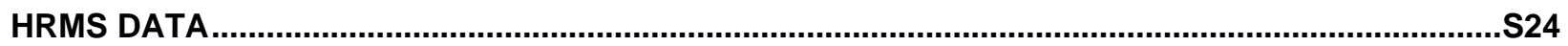

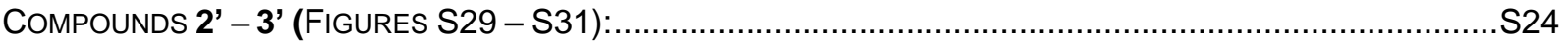

COMPUTATIONAL DATA

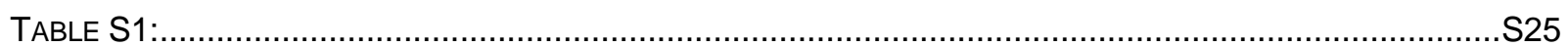

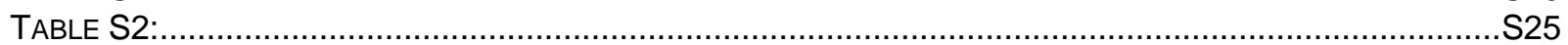

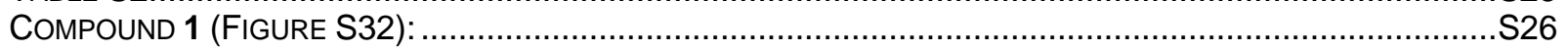




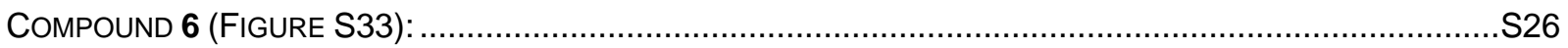

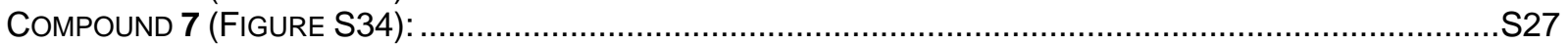

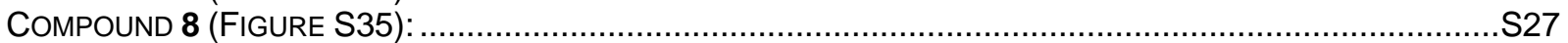

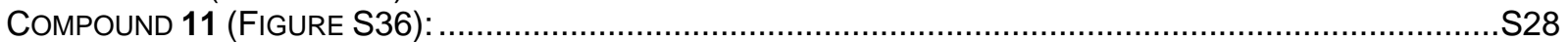

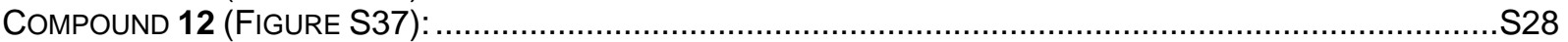

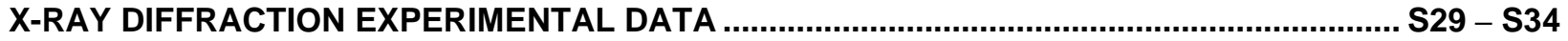

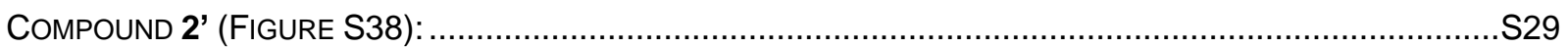

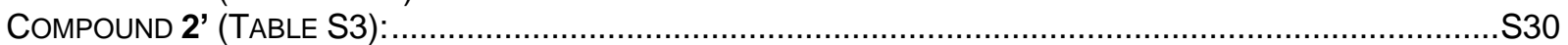

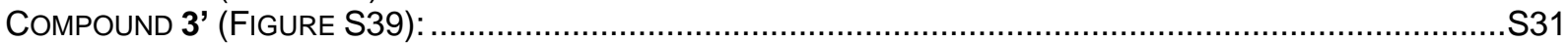

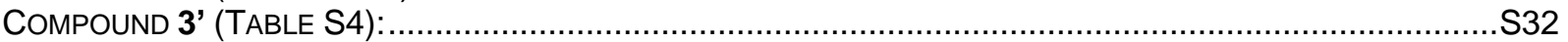

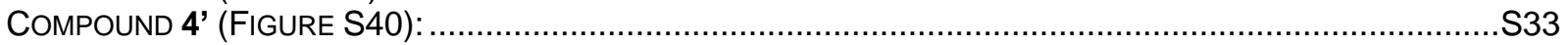

COMPOUND 4' (TABLE S5):

NMR SPECTRA FOR REPORTED COMPOUNDS. ............................................................. S35 - S71

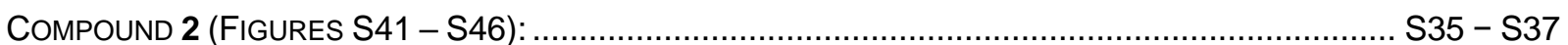

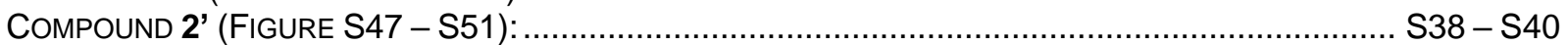

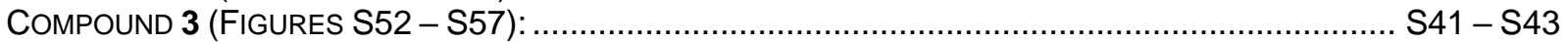

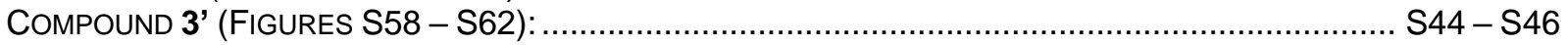

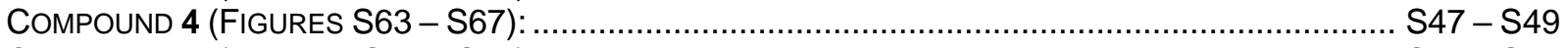

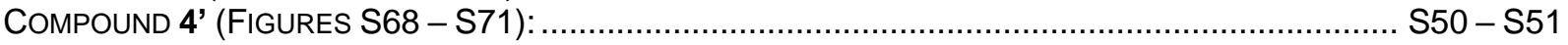

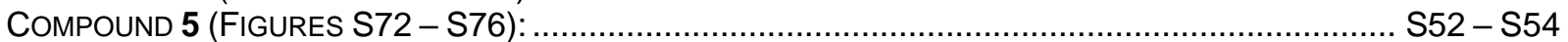

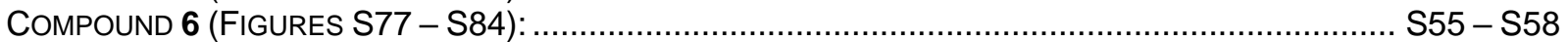

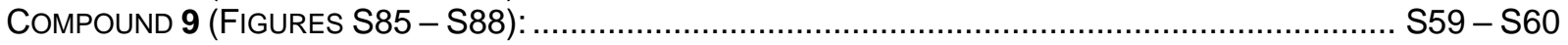

COMPOUND 15 (FIGURES S89 - S90):

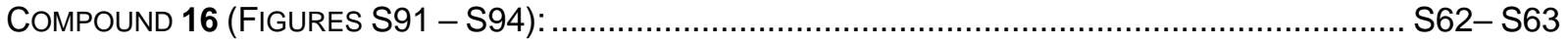

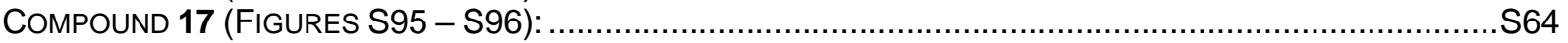

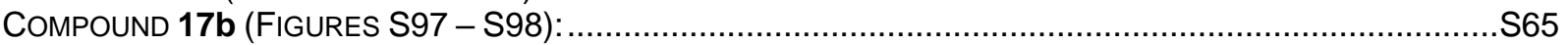

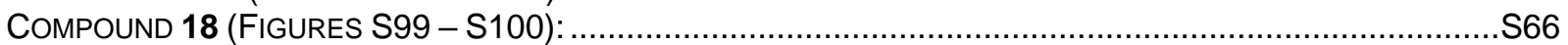

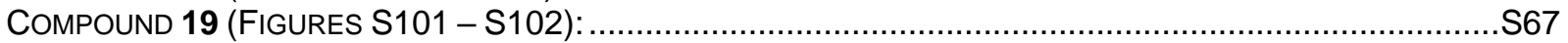

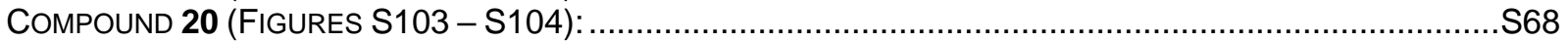

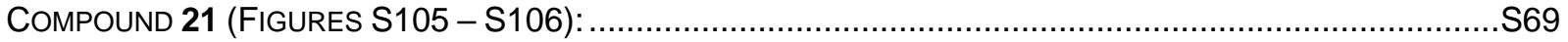

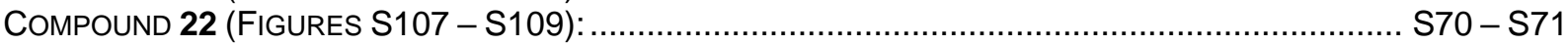

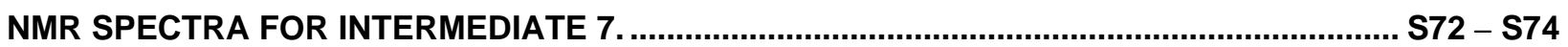

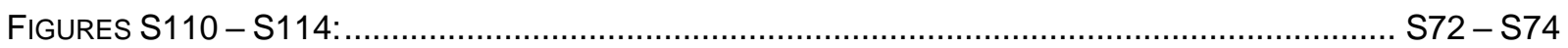

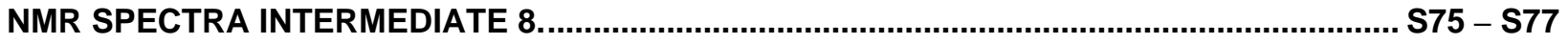

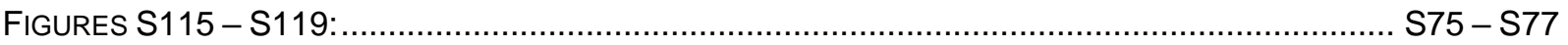

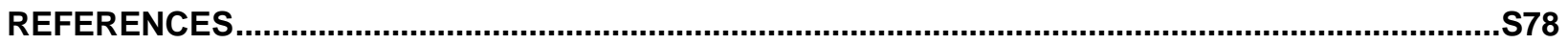




\section{General Methods}

All reactions were carried out in a nitrogen filled glove box under ambient temperatures and pressures unless otherwise specified. All liquid reagents and solvents were deoxygenated with three freeze-pump-thaw cycles before storing over $4 \AA$ molecular sieves. Molecular sieves were pre-activated by drying at $\sim 180^{\circ} \mathrm{C}$ under high vacuum for a minimum of 12 hours. orthoDifluorobenzene (DFB), diphenyl ether, hexyl methyl ether, and all nitriles used were dried by distillation over calcium hydride. Deuterated solvents and silanes were not dried prior to deoxygenation. Thin layer chromatography was conducted using SiliCyle Silica Gel 60 F254 plates and visualized with $254 \mathrm{~nm}$ UV light. Flash column chromatography was carried out using SilaFlash P60 40-63 um (230-400 mesh). Dry ice-acetone baths were used to cool reactions to $78{ }^{\circ} \mathrm{C}$. A liquid nitrogen cooled cold well, charged with copper beads, was used to freeze solutions in the glovebox.

Bis(tricyclohexylphosphine)palladium(0) was synthesized according to literature procedures ${ }^{1}$ or purchased from Strem Chemicals. The compounds $\left[\left(\mathrm{PCy}_{3}\right)_{2} \mathrm{PdSiMe} \mathrm{E}_{2} \mathrm{Et}\right]\left[\mathrm{B}\left(\mathrm{C}_{6} \mathrm{~F}_{5}\right)_{4}\right]^{2}$ (1) and $\mathrm{Na}\left[\mathrm{B}\left(\mathrm{C}_{6} \mathrm{~F}_{5}\right)_{4}\right]^{3}$ were prepared following established literature procedures. All other solvents and reagents were purchased from commercial sources: MilliporeSigma, TCI Chemicals, Strem Chemicals, Oakwood Chemicals, ACROS Organics, and Fisher Scientific.

Selected samples were analyzed with a Q Exactive HF-X (ThermoFisher, Bremen, Germany) mass spectrometer. Samples were introduced via a heated electrospray source (HESI) at a flow rate of $20 \mu \mathrm{L} / \mathrm{min}$. 100 time domain transients were averaged in the mass spectrum. ESI source conditions were set as: vaporizer temperature $35^{\circ} \mathrm{C}$, sheath gas (nitrogen) 8 arb, auxiliary gas (nitrogen) 0 arb, sweep gas (nitrogen) 0 arb, capillary temperature $320^{\circ} \mathrm{C}$, capillary voltage $320 \mathrm{~V}$ and funnel RF level $35 \mathrm{~V}$. The mass range was set to $150-2000 \mathrm{~m} / \mathrm{z}$. All measurements were recorded at a resolution setting of 120,000 . Solutions were analyzed at $0.1 \mathrm{mg} / \mathrm{mL}$ or less based on responsiveness to the ESI mechanism. Xcalibur (ThermoFisher, Breman, Germany) was used to analyze the data. Molecular formula assignments were determined with Molecular Formula Calculator ( $v$ 1.2.3). The mass-to-charge ratio of the cation of the reported organometallic salts are reported as $\mathrm{M}^{+}$. All observed species were singly charged, as verified by unit $\mathrm{m} / \mathrm{z}$ separation between mass spectral peaks corresponding to the ${ }^{12} \mathrm{C}$ and ${ }^{13} \mathrm{C}{ }^{12} \mathrm{C}_{\mathrm{c}-1}$ isotope for each elemental composition.

All NMR spectra were recorded on either Bruker Avance $600 \mathrm{MHz}, 500 \mathrm{MHz}$, or $400 \mathrm{MHz}$ spectrometers, or on Bruker Neo $600 \mathrm{MHz}$ or $400 \mathrm{MHz}$ spectrometers. Chemical shifts were recorded as $\delta$ values in ppm. The ${ }^{1} \mathrm{H},{ }^{29} \mathrm{Si}-\mathrm{HMBC}$ experiment was used to obtain ${ }^{29} \mathrm{Si}$ chemical shifts. Residual deuterated solvent proton or carbon resonances were used as internal references for NMR spectroscopy. ${ }^{4}$ The ${ }^{19} \mathrm{~F}\left\{{ }^{1} \mathrm{H}\right\}$ NMR spectra were referenced to either the solvent DFB fluorine resonances at $-138.6 \mathrm{ppm}$ or to the internally added fluorobenzene resonance at -113.0 ppm. Tetramethylsilane in $\mathrm{CD}_{2} \mathrm{Cl}_{2}$ or 1:5 DFB:toluene- $d_{8}$ was used as an external reference for the ${ }^{1} \mathrm{H},{ }^{29} \mathrm{Si}-\mathrm{HMBC}$ experiment. Due to overlap with solvent signals and their low intensities resulting from $J_{\mathrm{CF}}$, the carbon resonances of $\mathrm{B}\left(\mathrm{C}_{6} \mathrm{~F}_{5}\right)^{-}$are not reported. When applicable, NMR yields were calculated by integration with respect to the triphenylmethane signal ( $\delta 5.35 \mathrm{ppm})$, which was a by-product of the in situ generation of $\mathbf{1}$ and the silylnitrilium salts. Multiplicity is defined as follows: $s=$ singlet, $d=$ doublet, $t=$ triplet, $q=$ quartet, $p=$ pentet, hept $=$ heptet, $\mathrm{m}=$ multiplet, and $\mathrm{vt}=$ virtual triplet.

Elemental analysis was performed by Robertson Microlit Laboratories in Ledgewood N.J. When elemental analysis of isolated products was unsuccessful, high resolution NMR spectra to prove purity were provided (3', 4', and 9).

A Bruker SMART Apex II diffractometer was used to acquire X-ray crystallography data. 


\section{Reaction Procedures and Complex Syntheses}

Generation of $\left[\left(\mathrm{PCy}_{3}\right)_{2} \mathbf{P d}-\mathrm{SiMe}_{2} \mathrm{Et}\right]\left[\mathrm{B}\left(\mathrm{C}_{6} \mathrm{~F}_{5}\right)_{4}\right](1)$. Ethyldimethylsilane $(3.8 \mu \mathrm{L}, 0.0286 \mathrm{mmol})$ was added to a room temperature solution of $\left[\mathrm{Ph}_{3} \mathrm{C}\right]\left[\mathrm{B}\left(\mathrm{C}_{6} \mathrm{~F}_{5}\right)_{4}\right](24.0 \mathrm{mg}, 0.0260 \mathrm{mmol})$ and diphenylether $(4.1 \mu \mathrm{L}, 0.026 \mathrm{mmol})$ in $100 \mu \mathrm{L}$ DFB and $200 \mu \mathrm{L}$ toluene- $d_{8}$. The orange solution quickly turned nearly colorless, indicative of silyloxonium ion generation. The reaction mixture was transferred to a J. Young NMR tube and frozen in a liquid nitrogen cooled cold well before adding a solution of $\mathrm{Pd}\left(\mathrm{PCy}_{3}\right)_{2}(17.3 \mathrm{mg}, 0.026 \mathrm{mmol})$ in $300 \mu \mathrm{L}$ toluene- $d_{8}$. The reaction was then mixed while warming to room temperature and allowed to rest at ambient temperatures for $\sim 5$ minutes before using in subsequent experiments. The NMR spectroscopic data for 1 is reported elsewhere. ${ }^{2}$

General Procedure for Method a. A solution of in situ generated $1(0.0260 \mathrm{mmol})$, in $100 \mu \mathrm{L}$ DFB and $500 \mu \mathrm{L}$ toluene- $d_{8}$ was added to a J. Young NMR tube and frozen in a liquid nitrogen cooled cold well before nitrile $(0.026 \mathrm{mmol})$ was added. The reaction mixture was warmed to and mixed at $-78{ }^{\circ} \mathrm{C}$ before placing into the probe of a spectrometer pre-cooled to $-30^{\circ} \mathrm{C}$. After lowtemperature NMR spectroscopic analysis, the reaction was left to react at room temperature for 1 day. For reactions with aryl nitriles, the palladium-aryl products (2'-4') were isolated by silica gel flash column chromatography (10\% ethyl acetate in hexanes). X-ray quality crystals were obtained by slow evaporation from either ethyl acetate or dichloromethane.

[trans- $\left.\left(\mathrm{Me}_{2} \mathrm{EtSiNC}\right)\left(\mathrm{PCy}_{3}\right)_{2} \mathrm{Pd}\left(3,5-\left(\mathrm{CF}_{3}\right)_{2}-\mathrm{C}_{6} \mathrm{H}_{3}\right)\right]\left[\mathrm{B}\left(\mathrm{C}_{6} \mathrm{~F}_{5}\right)_{4}\right]$ (2). For the generation of 2, $4.4 \mu \mathrm{L}$ of 3,5- $\left(\mathrm{CF}_{3}\right)_{2}-\mathrm{C}_{6} \mathrm{H}_{3} \mathrm{CN}$ was used. ${ }^{1} \mathrm{H}$ NMR $\left(600 \mathrm{MHz}, 1: 5 \mathrm{DFB}:\right.$ Toluene- $\left.d_{8}\right) \delta 7.89$ (s, ortho ArH, $\left.2 \mathrm{H}\right)$, 7.55 (s, para ArH, 1H), $1.69(\mathrm{~m}, \mathrm{Cy}, 30 \mathrm{H}), 1.58(\mathrm{~d}, J=13.4 \mathrm{~Hz}, \mathrm{Cy}, 6 \mathrm{H}), 1.35$ (q, J = $12.7 \mathrm{~Hz}, \mathrm{Cy}$, $12 \mathrm{H}$ ), 1.05 (qt, $J=13.0,3.3 \mathrm{~Hz}, \mathrm{Cy}, 6 \mathrm{H}), 0.93-0.82\left(\mathrm{~m}, \mathrm{Cy}+\mathrm{SiCH}_{2} \mathrm{CH}_{3}, 15 \mathrm{H}\right), 0.65\left(\mathrm{q}, J_{\mathrm{HH}}=7.9\right.$ $\left.\mathrm{Hz}, \mathrm{SiCH}_{2} \mathrm{CH}_{3}, 2 \mathrm{H}\right), 0.26\left(\mathrm{~s}, \mathrm{SiMe}_{2}, 6 \mathrm{H}\right) .{ }^{13} \mathrm{C}\left\{{ }^{1} \mathrm{H}\right\} \mathrm{NMR}\left(151 \mathrm{MHz}, 1: 5 \mathrm{DFB}:\right.$ Toluene- $\left.d_{8}\right) \delta 162.2(\mathrm{t}$, $\left.J_{\mathrm{CP}}=15.0 \mathrm{~Hz}, \mathrm{CNSi}\right), 159.1$ (t, $J_{\mathrm{CP}}=5.1 \mathrm{~Hz}$, ipso $\mathrm{Ar}$ ), other aromatic resonances obscured, 35.7 (vt, $J_{\mathrm{CP}}=10.3 \mathrm{~Hz}, \mathrm{Cy}$ ), 30.6 (Cy), 27.8 (vt, $\left.J_{\mathrm{CP}}=5.5 \mathrm{~Hz}, \mathrm{Cy}\right), 26.4$ (Cy), $7.2\left(\mathrm{SiCH}_{2} \mathrm{CH}_{3}\right), 6.0$ $\left(\mathrm{SiCH}_{2} \mathrm{CH}_{3}\right),-3.1\left(\mathrm{SiMe}_{2}\right) .{ }^{31} \mathrm{P}\left\{{ }^{1} \mathrm{H}\right\} \mathrm{NMR}\left(202 \mathrm{MHz}, 1: 5 \mathrm{DFB}:\right.$ Toluene- $\left.d_{8}\right) \delta 28.4 .{ }^{19} \mathrm{~F}\left\{{ }^{1} \mathrm{H}\right\} \mathrm{NMR}(565$ $\mathrm{MHz}, 1: 5$ DFB:Toluene- $\left.d_{8}\right) \delta-63.2\left(\mathrm{~s}, \mathrm{CF}_{3}, 6 \mathrm{~F}\right),-131.8\left(\mathrm{~m}, \mathrm{~B}\left(\mathrm{C}_{6} \mathrm{~F}_{5}\right)_{4}, 8 \mathrm{~F}\right),-163.3\left(\mathrm{t}, \mathrm{J}_{\mathrm{FF}}=20.6 \mathrm{~Hz}\right.$, $\left.\mathrm{B}\left(\mathrm{C}_{6} \mathrm{~F}_{5}\right)_{4}, 4 \mathrm{~F}\right),-167.0\left(\mathrm{t}, \mathrm{J}_{\mathrm{FF}}=19.6 \mathrm{~Hz}, \mathrm{~B}\left(\mathrm{C}_{6} \mathrm{~F}_{5}\right)_{4}, 8 \mathrm{~F}\right) .{ }^{29} \mathrm{Si}$ from H,Si-HMBC $(100 \mathrm{MHz}, 500 \mathrm{MHz}$, 1:5 DFB:Toluene- $\left.d_{8}\right) \delta 19.1$

trans-(NC)(PCy $)_{2} \mathbf{P d}\left(3,5-\left(\mathbf{C F}_{3}\right)_{2}-\mathbf{C}_{6} \mathbf{H}_{3}\right)\left(\mathbf{2}^{\prime}\right) . \mathrm{Rf}=0.24$ (10\% ethyl acetate in hexanes), (17.9 $\mathrm{mg}$, $0.020 \mathrm{mmol}, 76 \%),{ }^{1} \mathrm{H}$ NMR $\left(400 \mathrm{MHz}, \mathrm{CD}_{2} \mathrm{Cl}_{2}\right) \delta 7.89$ (s, ortho $\left.\mathrm{ArH}, 2 \mathrm{H}\right), 7.39$ (s, para ArH, $\left.1 \mathrm{H}\right)$, $1.89(\mathrm{~m}, \mathrm{Cy}, 18 \mathrm{H}), 1.75$ (d, $J=13.0 \mathrm{~Hz}, \mathrm{Cy}, 12 \mathrm{H}), 1.66(\mathrm{~d}, J=12.9 \mathrm{~Hz}, \mathrm{Cy}, 6 \mathrm{H}), 1.54$ (q, $J=12.3$ $\mathrm{Hz}, \mathrm{Cy}, 12 \mathrm{H}$ ), 1.21 (qt, $J=12.3,3.4 \mathrm{~Hz}, \mathrm{Cy}, 6 \mathrm{H}$ ), 1.02 (q, $J=11.7 \mathrm{~Hz}, \mathrm{Cy}, 12 \mathrm{H}) .{ }^{13} \mathrm{C}\left\{{ }^{1} \mathrm{H}\right\} \mathrm{NMR}$ $\left(151 \mathrm{MHz}, \mathrm{CD}_{2} \mathrm{Cl}_{2}\right) \delta 165.6\left(\mathrm{t}, J_{\mathrm{CP}}=7.2 \mathrm{~Hz}\right.$, ipso Ar), $138.7(\mathrm{Ar}), 137.5$ (t, $J_{\mathrm{CP}}=16.9 \mathrm{~Hz}, \mathrm{CN}$ ), 128.2 (q, $\left.J_{\mathrm{CF}}=31.3 \mathrm{~Hz}, \mathrm{Ar}\right), 124.7\left(\mathrm{q}, J_{\mathrm{CF}}=272.9 \mathrm{~Hz}, \mathrm{CF}_{3}\right), 116.0(\mathrm{~m}, \mathrm{Ar}), 35.2\left(\mathrm{vt}, \mathrm{J}_{\mathrm{CP}}=10.5 \mathrm{~Hz}\right.$, Cy), 30.3 (Cy), 27.9 (vt, $\left.J_{\mathrm{CP}}=5.4 \mathrm{~Hz}, \mathrm{Cy}\right), 26.8$ (Cy). ${ }^{31} \mathrm{P}\left\{{ }^{1} \mathrm{H}\right\} \mathrm{NMR}\left(162 \mathrm{MHz}, \mathrm{CD}_{2} \mathrm{Cl}_{2}\right) \delta 27.9$, ${ }^{19} \mathrm{~F}\left\{{ }^{1} \mathrm{H}\right\}$ NMR $\left(565 \mathrm{MHz}, \mathrm{CD}_{2} \mathrm{Cl}_{2}\right) \delta-62.2\left(\mathrm{~s}, \mathrm{CF}_{3}, 6 \mathrm{~F}\right)$. Anal. Calcd for $\mathrm{C}_{44} \mathrm{H}_{69} \mathrm{NF}_{6} \mathrm{P}_{2} \mathrm{Pd}: \mathrm{C}, 59.63$; $\mathrm{H}, 7.67 ; \mathrm{N}, 1.55$. Found: $\mathrm{C}, 59.44 ; \mathrm{H}, 7.78 ; \mathrm{N}, 1.37$. HRMS-ESI $(\mathrm{m} / \mathrm{z}):[\mathrm{M}-\mathrm{CN}]^{+}$calcd for $\mathrm{C}_{44} \mathrm{H}_{69} \mathrm{~F}_{6} \mathrm{P}_{2} \mathrm{Pd}$, 879.374479; found, 879.37817.

[trans- $\left.\left(\mathrm{Me}_{2} \mathrm{EtSiNC}\right)\left(\mathrm{PCy}_{3}\right)_{2} \mathrm{Pd}\left(3-\mathrm{F}-\mathrm{C}_{6} \mathrm{H}_{4}\right)\right]\left[\mathrm{B}\left(\mathrm{C}_{6} \mathrm{~F}_{5}\right)_{4}\right]$ (3). For the generation of 3, $2.8 \mu \mathrm{L}$ of 3-F$\mathrm{C}_{6} \mathrm{H}_{4} \mathrm{CN}$ was used. ${ }^{1} \mathrm{H}$ NMR $\left(600 \mathrm{MHz}, 1: 5 \mathrm{DFB}: T o l u e n e-d_{8}\right) \delta$ Aromatic resonances obscured, 1.69 (m, Cy, 30H), 1.61 (d, J=13.4 Hz, Cy, 6H), 1.35 (m, Cy, 12H), 1.09 (qt, J = 13.0, 3.4 Hz, Cy, 6H), $0.94(\mathrm{~m}$, Cy $12 \mathrm{H}), 0.89\left(\mathrm{t}, \mathrm{J}_{\mathrm{HH}}=7.9 \mathrm{~Hz}, \mathrm{SiCH}_{2} \mathrm{CH}_{3}, 3 \mathrm{H}\right), 0.64\left(\mathrm{q}, \mathrm{J}_{\mathrm{HH}}=8.0 \mathrm{~Hz}, \mathrm{SiCH}_{2} \mathrm{CH}_{3}\right.$, $2 \mathrm{H}), 0.24\left(\mathrm{~s}, \mathrm{SiMe}_{2}, 6 \mathrm{H}\right) .{ }^{13} \mathrm{C}\left\{{ }^{1} \mathrm{H}\right\}$ NMR (151 MHz, 1:5 DFB:Toluene-d $\left.d_{8}\right) \delta 164.2\left(\mathrm{t}, \mathrm{J}_{\mathrm{CP}}=15.3 \mathrm{~Hz}\right.$, CNSi), 162.8 (d, $\left.J_{\mathrm{CF}}=250.9 \mathrm{~Hz}, \mathrm{Ar}-\mathrm{F}\right), 157.2$ (q, $J_{\mathrm{CP}}=4.5 \mathrm{~Hz}$, ipso Ar), 110.7 (d, $J_{\mathrm{CF}}=20.5 \mathrm{~Hz}$, ArF), other aromatic resonances obscured, 35.5 (vt, $\left.J_{\mathrm{CP}}=10.4 \mathrm{~Hz}, \mathrm{Cy}\right), 30.7$ (Cy), 27.8 (q, J $J_{\mathrm{CP}}=$ $5.1 \mathrm{~Hz}, \mathrm{Cy}), 26.52(\mathrm{Cy}), 7.3\left(\mathrm{SiCH}_{2} \mathrm{CH}_{3}\right), 6.0\left(\mathrm{SiCH}_{2} \mathrm{CH}_{3}\right),-3.0\left(\mathrm{SiMe}_{2}\right) .{ }^{31} \mathrm{P}\left\{{ }^{1} \mathrm{H}\right\} \mathrm{NMR}(202 \mathrm{MHz}$, 1:5 DFB:Toluene- $\left.d_{8}\right) \delta$ 27.2. ${ }^{19} \mathrm{~F}\left\{{ }^{1} \mathrm{H}\right\}$ NMR (565 MHz, 1:5 DFB:Toluene- $\left.d_{8}\right) \delta-113.0(\mathrm{~s}, \mathrm{ArF}, 1 \mathrm{~F})$, $-131.8\left(\mathrm{~m}, \mathrm{~B}\left(\mathrm{C}_{6} \mathrm{~F}_{5}\right)_{4}, 8 \mathrm{~F}\right),-163.3\left(\mathrm{t}, \mathrm{J}_{\mathrm{FF}}=20.6 \mathrm{~Hz}, \mathrm{~B}\left(\mathrm{C}_{6} \mathrm{~F}_{5}\right)_{4}, 4 \mathrm{~F}\right),-167.0\left(\mathrm{t}, J_{\mathrm{FF}}=19.5 \mathrm{~Hz}, \mathrm{~B}\left(\mathrm{C}_{6} \mathrm{~F}_{5}\right)_{4}\right.$, $8 \mathrm{~F}) .{ }^{29} \mathrm{Si}$ from H,Si-HMBC (100 MHz, $500 \mathrm{MHz}, 1: 5 \mathrm{DFB}$ :Toluene- $\left.d_{8}\right) \delta$ 17.9. 
trans-(NC)(PCy $)_{2} \mathbf{P d}\left(3-F-\mathbf{C}_{6} \mathbf{H}_{4}\right)\left(\mathbf{3}^{\prime}\right) . \mathrm{Rf}=0.24$ (10\% ethyl acetate in hexanes), (19.2 $\mathrm{mg}, 0.024$ mmol, 94\%). ${ }^{1} \mathrm{H}$ NMR (400 MHz, $\left.\mathrm{CD}_{2} \mathrm{Cl}_{2}\right) \delta 7.13(\mathrm{~d}, J=7.4 \mathrm{~Hz}, \mathrm{ArH}, 1 \mathrm{H}), 7.06$ (dd, $J=10.0,2.7$ $\mathrm{Hz}, \mathrm{ArH}, 1 \mathrm{H}), 6.92(\mathrm{q}, J=7.3 \mathrm{~Hz}, \mathrm{ArH}, 1 \mathrm{H}), 6.52$ (td, $J=8.8,2.6 \mathrm{~Hz}, \mathrm{ArH}, 1 \mathrm{H}), 1.92(\mathrm{~m}, \mathrm{Cy}, 18 \mathrm{H})$, 1.75 (m, Cy, 12H), 1.65 (m, Cy, 6H), 1.55 (q, $J=12.1 \mathrm{~Hz}$, Cy, 12H), 1.21 (qt, $J=11.7,2.9 \mathrm{~Hz}$, Cy, 6H), 1.07 (q, J=12.4 Hz, Cy, $12 \mathrm{H}) .{ }^{13} \mathrm{C}\left\{{ }^{1} \mathrm{H}\right\} \mathrm{NMR}\left(101 \mathrm{MHz}, \mathrm{CD}_{2} \mathrm{Cl}_{2}\right) \delta 164.1$ (td, $J=6.5,1.9$ $\mathrm{Hz}$, ipso Ar), 162.0 (d, $\left.J_{\mathrm{CF}}=246.9 \mathrm{~Hz}, \mathrm{Ar}-\mathrm{F}\right), 139.5$ (t, $\left.J_{\mathrm{CP}}=16.6 \mathrm{~Hz}, \mathrm{CN}\right), 134.7$ (d, J $J_{\mathrm{CF}}=2.7 \mathrm{~Hz}$, Ar), 127.3 (d, J $\left.J_{C F}=6.7 \mathrm{~Hz}, A r\right), 124.6$ (dt, $\left.J=15.5,2.7 \mathrm{~Hz}, \mathrm{Ar}\right), 108.3\left(\mathrm{~d}, J_{\mathrm{CF}}=20.7 \mathrm{~Hz}, \mathrm{Ar}\right.$ ), 35.1 (vt, $J_{\mathrm{CP}}=10.4 \mathrm{~Hz}, \mathrm{Cy}$ ), 30.4 (Cy), 28.0 (td, $\left.J=6.3,3.1 \mathrm{~Hz}, \mathrm{Cy}\right), 26.9$ (Cy). ${ }^{31} \mathrm{P}\left\{{ }^{1} \mathrm{H}\right\} \mathrm{NMR}(162 \mathrm{MHz}$, $\left.\mathrm{CD}_{2} \mathrm{Cl}_{2}\right) \delta$ 27.0. ${ }^{19} \mathrm{~F}\left\{{ }^{1} \mathrm{H}\right\}$ NMR (565 MHz, $\left.\mathrm{CD}_{2} \mathrm{Cl}_{2}\right) \delta-116.56$ (s, Ar-F, 1F). HRMS-ESI (m/z): [M $\mathrm{CN}]^{+}$calcd for $\mathrm{C}_{42} \mathrm{H}_{70} \mathrm{FP}_{2} \mathrm{Pd}, 761.396607$; found, 761.39314 .

[trans-(Me $\left.\left.{ }_{2} \mathrm{EtSiNC}\right)\left(\mathrm{PCy}_{3}\right)_{2} \mathrm{Pd}(\mathrm{Ph})\right]\left[\mathrm{B}\left(\mathrm{C}_{6} \mathrm{~F}_{5}\right)_{4}\right](4)$. For the generation of $4,2.7 \mu \mathrm{L}$ of benzonitrile was used. ${ }^{1} \mathrm{H}$ NMR $\left(600 \mathrm{MHz}, 1: 5\right.$ DFB:Toluene- $\left.d_{8}\right) \delta 7.13\left(\mathrm{~d}, \mathrm{~J}_{\mathrm{HH}}=7.5 \mathrm{~Hz}, \mathrm{ArH}, 2 \mathrm{H}\right)$, other aromatic resonances obscured, $1.71(\mathrm{~m}, \mathrm{Cy}, 30 \mathrm{H}), 1.61(\mathrm{~d}, J=13.5 \mathrm{~Hz}, \mathrm{Cy}, 6 \mathrm{H}), 1.37(\mathrm{q}, J=12.6$ $\mathrm{Hz}, \mathrm{Cy}, 12 \mathrm{H}$ ), 1.10 (qt, $J=13.1,3.6 \mathrm{~Hz}, \mathrm{Cy}, 6 \mathrm{H}), 0.97-0.88\left(\mathrm{~m}, \mathrm{Cy}+\mathrm{SiCH}_{2} \mathrm{CH}_{3}, 12 \mathrm{H}\right.$ ), 0.64 (q, $\left.J_{\mathrm{HH}}=7.9 \mathrm{~Hz}, \mathrm{SiCH}_{2} \mathrm{CH}_{3}, 2 \mathrm{H}\right), 0.24\left(\mathrm{~s}, \mathrm{SiMe}_{2}, 6 \mathrm{H}\right) \cdot{ }^{13} \mathrm{C}\left\{{ }^{1} \mathrm{H}\right\} \mathrm{NMR}\left(151 \mathrm{MHz}, 1: 5 \mathrm{DFB}:\right.$ Toluene- $\left.d_{8}\right)$ $\delta 165.3\left(\mathrm{t}, J_{\mathrm{CP}}=15.0 \mathrm{~Hz}, \mathrm{CNSi}\right), 154.3\left(\mathrm{t}, J_{\mathrm{CP}}=3.9 \mathrm{~Hz}\right.$, ipso Ar), other aromatic resonances obscured, 35.5 (vt, $J_{C P}=10.4 \mathrm{~Hz}, \mathrm{Cy}$ ), 30.7 (Cy), 27.8 (vt, $J_{\mathrm{CP}}=5.5 \mathrm{~Hz}, \mathrm{Cy}$ ), 26.6 (Cy), 7.3 $\left(\mathrm{SiCH}_{2} \mathrm{CH}_{3}\right), 6.1\left(\mathrm{SiCH}_{2} \mathrm{CH}_{3}\right),-3.0\left(\mathrm{SiMe}_{2}\right) .{ }^{31} \mathrm{P}\left\{{ }^{1} \mathrm{H}\right\} \mathrm{NMR}\left(202 \mathrm{MHz}, 1: 5 \mathrm{DFB}: T o l u e n e-d_{8}\right) \delta$ 26.7. ${ }^{29} \mathrm{Si}$ from H,Si-HMBC (100 MHz, $500 \mathrm{MHz}, 1: 5 \mathrm{DFB}$ :Toluene- $\left.d_{8}\right) \delta 17.4$.

trans-(NC)(PCy $)_{2} \mathbf{P d}\left(\mathbf{C}_{6} \mathbf{H}_{5}\right)\left(\mathbf{4}^{\prime}\right) . \mathrm{Rf}=0.22$ (10\% ethyl acetate in hexanes), (15.3 $\mathrm{mg}, 0.020 \mathrm{mmol}$, $76 \%),{ }^{1} \mathrm{H}$ NMR $\left(400 \mathrm{MHz}, \mathrm{CD}_{2} \mathrm{Cl}_{2}\right) \delta 7.31\left(\mathrm{~d}, J_{\mathrm{HH}}=7.3 \mathrm{~Hz}, \mathrm{ArH}, 2 \mathrm{H}\right), 6.91\left(\mathrm{t}, J_{\mathrm{HH}}=7.3 \mathrm{~Hz}, \mathrm{ArH}\right.$, 2H), $6.79\left(\mathrm{t}, \mathrm{J}_{\mathrm{HH}}=7.3 \mathrm{~Hz}, \mathrm{ArH}, 1 \mathrm{H}\right), 1.98-1.88(\mathrm{~m}, \mathrm{Cy}, 18 \mathrm{H}), 1.73(\mathrm{~d}, J=12.8 \mathrm{~Hz}, \mathrm{Cy}, 12 \mathrm{H}), 1.64$ (m, Cy, 6H), 1.54 (q, J=12.2 Hz, Cy, 12H), 1.20 (qt, $J=12.2,3.6, C y, 6 \mathrm{H}$ ), 1.06 (q, J = 12.5, Cy, $12 \mathrm{H}) .{ }^{13} \mathrm{C}\left\{{ }^{1} \mathrm{H}\right\}$ NMR $\left(101 \mathrm{MHz}, \mathrm{CD}_{2} \mathrm{Cl}_{2}\right) \delta 160.1$ (t, $J_{\mathrm{CP}}=5.8 \mathrm{~Hz}$, ipso $\left.\mathrm{Ar}\right), 140.7$ (t, $J_{\mathrm{CP}}=17.2 \mathrm{~Hz}$, $\mathrm{CN}$ ), 139.0 (t, $J_{\mathrm{CP}}=2.8 \mathrm{~Hz}, \mathrm{Ar}$ ), $126.9(\mathrm{Ar}), 121.8(\mathrm{Ar}), 35.0$ (vt, $J_{\mathrm{CP}}=10.3 \mathrm{~Hz}, \mathrm{Cy}$ ), $30.4(\mathrm{Cy}), 28.0$ (vt, $\left.J_{\mathrm{CP}}=5.4 \mathrm{~Hz}, \mathrm{Cy}\right), 26.9(\mathrm{Cy}) .{ }^{31} \mathrm{P}\left\{{ }^{1} \mathrm{H}\right\}$ NMR (162 MHz, $\left.\mathrm{CD}_{2} \mathrm{Cl}_{2}\right) \delta$ 26.6. HRMS-ESI $(\mathrm{m} / \mathrm{z}):[\mathrm{M}-$ $\mathrm{CN}]^{+}$calcd for $\mathrm{C}_{42} \mathrm{H}_{71} \mathrm{P}_{2} \mathrm{Pd}$, 743.406032; found, 743.40308.

[trans-(NC)(PCy $\left.)_{2} \mathbf{P d}(\mathbf{H})\right]\left[B\left(\mathrm{C}_{6} \mathrm{~F}_{5}\right)_{4}\right](5)$. For the generation of $5,2.3 \mu \mathrm{L}$ of isobutyronitrile was used. ${ }^{1} \mathrm{H}$ NMR $\left(600 \mathrm{MHz}, 1: 5 \mathrm{DFB}: T o l u e n e-d_{8}\right) \delta 1.85(\mathrm{t}, J=12.2 \mathrm{~Hz}, \mathrm{Cy}, 6 \mathrm{H}), 1.78(\mathrm{~d}, J=12.7$ $\mathrm{Hz}, \mathrm{Cy}, 12 \mathrm{H}), 1.71$ (d, J = 12.6 Hz, Cy, 12H), $1.64(\mathrm{~d}, J=12.2 \mathrm{~Hz}, \mathrm{Cy}, 6 \mathrm{H}), 1.29$ (qd, $J=13.9$, 13.0, 7.5 Hz, Cy, 12H), $1.13(\mathrm{~m}, \mathrm{Cy}, 18 \mathrm{H}), 0.88\left(\mathrm{t}, \mathrm{J}_{\mathrm{HH}}=7.9 \mathrm{~Hz}, \mathrm{SiCH}_{2} \mathrm{CH}_{3}, 3 \mathrm{H}\right), 0.58\left(\mathrm{q}, J_{\mathrm{HH}}=7.9\right.$ $\left.\mathrm{Hz}, \mathrm{SiCH} \mathrm{CH}_{3}, 2 \mathrm{H}\right), 0.18\left(\mathrm{~s}, \mathrm{SiMe}_{3}, 6 \mathrm{H}\right),-9.17\left(\mathrm{t}, \mathrm{J}_{\mathrm{HP}}=8.8 \mathrm{~Hz}, \mathrm{Pd}-\mathrm{H}, 1 \mathrm{H}\right) .{ }^{13} \mathrm{C}\left\{{ }^{1} \mathrm{H}\right\} \mathrm{NMR}(151$ $\mathrm{MHz}, 1: 5$ DFB:Toluene- $\left.d_{8}\right) \delta 167.8$ (t, $\left.J_{\mathrm{CP}}=11.1 \mathrm{~Hz}, \mathrm{CNSi}\right), 36.2\left(\mathrm{vt}, J_{\mathrm{CP}}=11.2 \mathrm{~Hz}, \mathrm{Cy}\right), 30.9$ (Cy), 27.5 (vt, $\left.J_{\mathrm{CP}}=5.7 \mathrm{~Hz}, \mathrm{Cy}\right), 26.5(\mathrm{Cy}), 7.4\left(\mathrm{SiCH} \mathrm{CH}_{3}\right), 6.0\left(\mathrm{SiCH}_{2} \mathrm{CH}_{3}\right),-3.0\left(\mathrm{SiMe}_{2}\right) .{ }^{31} \mathrm{P}\left\{{ }^{1} \mathrm{H}\right\}$ NMR (202 MHz, 1:5 DFB:Toluene- $\left.d_{8}\right) \delta$ 49.1. ${ }^{29} \mathrm{Si}$ from H,Si-HMBC (100 MHz, $500 \mathrm{MHz}, 1: 5$ DFB:Toluene- $\left.d_{8}\right) \delta 16.8$.

$\left[\left(\mathrm{PCy}_{3}\right)_{2} \mathrm{Pd}\left\{\mathrm{C}=\left(\mathrm{NSiMe}_{2} \mathrm{Et}\right) \mathrm{CH}_{3}\right\}\right]\left[\mathrm{B}\left(\mathrm{C}_{6} \mathrm{~F}_{5}\right)_{4}\right]$ (6). For the generation of $6,1.4 \mu \mathrm{L}$ of acetonitrile was used. ${ }^{1} \mathrm{H}$ NMR $\left(600 \mathrm{MHz}, 1: 5 \mathrm{DFB}: T o l u e n e-d_{8}\right) \delta 2.31\left(\mathrm{t}, \mathrm{J}_{\mathrm{HP}}=2.3 \mathrm{~Hz}, \mathrm{C}=(\mathrm{NSi}) \mathrm{CH}_{3}, 3 \mathrm{H}\right), 1.84$ (t, $J=11.6 \mathrm{~Hz}, \mathrm{Cy}, 6 \mathrm{H}), 1.78(\mathrm{~d}, J=11.8 \mathrm{~Hz}, \mathrm{Cy}, 12 \mathrm{H}), 1.72(\mathrm{~d}, J=11.8 \mathrm{~Hz}, \mathrm{Cy}, 12 \mathrm{H}), 1.64(\mathrm{~d}, J=$ $12.2 \mathrm{~Hz}, \mathrm{Cy}, 6 \mathrm{H}), 1.34(\mathrm{q}, J=12.4 \mathrm{~Hz}, \mathrm{Cy}, 12 \mathrm{H}), 1.13(\mathrm{~m}, \mathrm{Cy}, 18 \mathrm{H}), 0.80$ (t, JHH $=7.9 \mathrm{~Hz}$, $\mathrm{SiCH}_{2} \mathrm{CH}_{3}, 3 \mathrm{H}$ ), 0.60 (d, $\left.J_{\mathrm{HH}}=7.9 \mathrm{~Hz}, \mathrm{SiCH}_{2} \mathrm{CH}_{3}, 2 \mathrm{H}\right), 0.18$ (s, SiMe, $\left.6 \mathrm{H}\right) .{ }^{13} \mathrm{C}\left\{{ }^{1} \mathrm{H}\right\},{ }^{13} \mathrm{C}-\mathrm{DEPT} 135$, $\mathrm{H}, \mathrm{C}-\mathrm{HMBC}$ NMR (101 MHz, 1:5 DFB:Toluene- $\left.d_{8}, 0^{\circ} \mathrm{C}\right) \delta 176.7(C=\mathrm{N}), 35.0\left(\mathrm{~d}, J_{\mathrm{HP}}=15.3 \mathrm{~Hz}\right.$, Cy), 30.9 (Cy), 27.7 (d, J JP = 10.0 Hz, Cy), 26.4 (Cy), 20.7 (t, J JP = 16.9 Hz, C=(N)CH $\left.\mathrm{N}_{3}\right), 7.9$ $\left(\mathrm{SiCH}_{2} \mathrm{CH}_{3}\right), 6.3\left(\mathrm{SiCH}_{2} \mathrm{CH}_{3}\right)$, -2.3 $\left(\mathrm{SiMe}_{2}\right) .{ }^{31} \mathrm{P}\left\{{ }^{1} \mathrm{H}\right\}$ NMR $\left(202 \mathrm{MHz}, 1: 5 \mathrm{DFB}: T o l u e n e-d_{8}\right) \delta 37.4$. ${ }^{29} \mathrm{Si}$ from H,Si-HMBC (100 MHz, $\left.500 \mathrm{MHz}, 1: 5 \mathrm{DFB}: T o l u e n e-d_{8}\right) \delta 13.2$.

Procedure for the Generation of SilyInitrilium Salts. Triethylsilane ( $4.6 \mu \mathrm{L}, 0.029 \mathrm{mmol})$ or ethyldimethylsilane $(3.8 \mu \mathrm{L}, 0.029 \mathrm{mmol})$ was added to a room temperature solution of $\left[\mathrm{Ph}_{3} \mathrm{C}\right]\left[\mathrm{B}\left(\mathrm{C}_{6} \mathrm{~F}_{5}\right)_{4}\right](24 \mathrm{mg}, 0.026 \mathrm{mmol})$ and diphenyl ether $(4.1 \mu \mathrm{L}, 0.026 \mathrm{mmol})$ in $100 \mu \mathrm{L}$ of DFB and $500 \mu \mathrm{L}$ of toluene- $d_{8}$. The orange solution quickly turned nearly colorless, indicative of 
silyloxonium ion generation. Nitrile $(0.026 \mathrm{mmol})$ was then added to the in situ generated silyloxonium ion to produce the silylnitrilium ion.

$\left[\left(\left(\mathrm{CH}_{3}\right)_{2} \mathbf{C H}\right)_{3} \mathrm{SiNCCH}_{3}\right]\left[\mathrm{B}\left(\mathbf{C}_{6} \mathbf{F}_{5}\right)_{4}\right]$ (15). ${ }^{1} \mathrm{H}$ NMR $\left(500 \mathrm{MHz}, 1: 5 \mathrm{DFB}:\right.$ Toluene- $\left.d_{8}\right) \delta 1.51$ (s, NCMe, $3 \mathrm{H}), 0.87\left(\mathrm{~m}, \mathrm{CH}\left(\mathrm{CH}_{3}\right)_{2}, 3 \mathrm{H}\right), 0.76\left(\mathrm{~d}, \mathrm{~J}_{\mathrm{HH}}=7.2 \mathrm{~Hz}, \mathrm{CH}\left(\mathrm{CH}_{3}\right)_{2}, 18 \mathrm{H}\right) .{ }^{29} \mathrm{Si}$ from H,Si-HMBC (80 $\mathrm{MHz}, 400 \mathrm{MHz}, 1: 5 \mathrm{DFB}:$ Toluene- $\left.d_{8}\right) \delta 38.8$.

[EtMe $\left.{ }_{2} \operatorname{SiNC}\left(\mathbf{3}, \mathbf{5}-\left(\mathbf{C F}_{3}\right)_{2}-\mathbf{C}_{6} \mathbf{H}_{3}\right)\right]\left[B\left(\mathbf{C}_{6} \mathbf{F}_{5}\right)_{4}\right]$ (17). ${ }^{1} \mathrm{H}$ NMR $\left(400 \mathrm{MHz}, 1: 5 \mathrm{DFB}: T o l u e n e-d_{8}\right) \delta 7.95$ (s, $\mathrm{ArH}, 1 \mathrm{H}), 7.81(\mathrm{~s}, \mathrm{ArH}, 2 \mathrm{H})$, other aromatic resonances obscured, 0.83 (t, $\mathrm{JHH}_{\mathrm{HH}}=7.8 \mathrm{~Hz}, \mathrm{SiCH}_{2} \mathrm{CH}_{3}$, $3 \mathrm{H}), 0.66$ (qd, $\left.J=7.3,1.4 \mathrm{~Hz}, \mathrm{SiCH}_{2} \mathrm{CH}_{3}, 2 \mathrm{H}\right), 0.28\left(\mathrm{~s}, \mathrm{SiMe}_{2}, 6 \mathrm{H}\right) .{ }^{29} \mathrm{Si}$ from H,Si-HMBC $(80 \mathrm{MHz}$, $400 \mathrm{MHz}, 1: 5 \mathrm{DFB}:$ Toluene- $\left.d_{8}\right) \delta 44.4$.

$\left[\mathrm{Et}_{3} \operatorname{SiNC}\left(\mathbf{3}, \mathbf{5}-\left(\mathrm{CF}_{3}\right)_{2}-\mathrm{C}_{6} \mathbf{H}_{3}\right)\right]\left[\mathrm{B}\left(\mathbf{C}_{6} \mathbf{F}_{5}\right)_{4}\right]$ (17b). ${ }^{1} \mathrm{H}$ NMR $\left(400 \mathrm{MHz}, 1: 5 \mathrm{DFB}: T o l u e n e-d_{8}\right) \delta 7.97$ (s, $\mathrm{ArH}, 1 \mathrm{H}), 7.89(\mathrm{~s}, \mathrm{ArH}, 2 \mathrm{H}), 0.85\left(\mathrm{t}, \mathrm{J}_{\mathrm{HH}}=7.7 \mathrm{~Hz}, \mathrm{SiCH}_{2} \mathrm{CH}_{3}, 9 \mathrm{H}\right), 0.70$ (qd, J = 7.3, $1.6 \mathrm{~Hz}$, $\left.\mathrm{SiCH}_{2} \mathrm{CH}_{3}, 6 \mathrm{H}\right) .{ }^{29} \mathrm{Si}$ from H,Si-HMBC $\left(80 \mathrm{MHz}, 400 \mathrm{MHz}, 1: 5 \mathrm{DFB}\right.$ :Toluene-d $\left.d_{8}\right) \delta 45.8$.

[EtMe $\left.{ }_{2} \mathbf{S i N C}\left(3-\mathbf{F}_{3}-\mathbf{C}_{6} \mathbf{H}_{4}\right)\right]\left[B\left(\mathbf{C}_{6} \mathbf{F}_{5}\right)_{4}\right]$ (18). ${ }^{1} \mathrm{H}$ NMR (400 MHz, 1:5 DFB:Toluene- $\left.d_{8}\right) \delta$ Aromatic resonances obscured, $0.78\left(\mathrm{t}, \mathrm{J}_{\mathrm{HH}}=7.9 \mathrm{~Hz}, \mathrm{SiCH}_{2} \mathrm{CH}_{3}, 3 \mathrm{H}\right.$ ), $0.57\left(\mathrm{q}, \mathrm{J}_{\mathrm{HH}}=7.8 \mathrm{~Hz}, \mathrm{SiCH}_{2} \mathrm{CH}_{3}\right.$, 2H), 0.19 (s, SiMe $2,6 \mathrm{H}) .{ }^{29} \mathrm{Si}$ from H,Si-HMBC $\left(80 \mathrm{MHz}, 400 \mathrm{MHz}, 1: 5 \mathrm{DFB}:\right.$ Toluene- $\left.d_{8}\right) \delta 40.1$.

[EtMe $\left.{ }_{2} \operatorname{SiNC}\left(\mathbf{C}_{6} \mathrm{H}_{5}\right)\right]\left[\mathrm{B}\left(\mathrm{C}_{6} \mathbf{F}_{5}\right)_{4}\right]$ (19). ${ }^{1} \mathrm{H}$ NMR $\left(400 \mathrm{MHz}, 1: 5\right.$ DFB:Toluene- $\left.d_{8}\right) \delta$ Aromatic resonances obscured, $0.77\left(\mathrm{t}, \mathrm{J}_{\mathrm{HH}}=7.9 \mathrm{~Hz}, \mathrm{SiCH}_{2} \mathrm{CH}_{3}, 3 \mathrm{H}\right), 0.54\left(\mathrm{q}, \mathrm{J}_{\mathrm{H}}=8.1 \mathrm{~Hz}, \mathrm{SiCH}_{2} \mathrm{CH}_{3}\right.$, 2H), 0.16 (s, $\left.\mathrm{SiMe}_{2} 6 \mathrm{H}\right) .{ }^{29} \mathrm{Si}$ from H,Si-HMBC $\left(80 \mathrm{MHz}, 400 \mathrm{MHz}, 1: 5 \mathrm{DFB}: T o l u e n e-d_{8}\right) \delta 37.9$.

[EtMe $\left.{ }_{2} \mathrm{SiNCCH}\left(\mathrm{CH}_{3}\right)_{2}\right]\left[\mathrm{B}\left(\mathrm{C}_{6} \mathrm{~F}_{5}\right)_{4}\right](20) .{ }^{1} \mathrm{H}$ NMR $\left(400 \mathrm{MHz}, 1: 5 \mathrm{DFB}\right.$ :Toluene-d $\left.d_{8}\right) 2.29$ (hept, $\mathrm{J}_{\mathrm{HH}}$ $\left.=7.1 \mathrm{~Hz} \mathrm{CH}\left(\mathrm{CH}_{3}\right)_{2}, 1 \mathrm{H}\right), 0.80\left(\mathrm{~d}, \mathrm{~J}_{\mathrm{HH}}=7.1 \mathrm{~Hz}, \mathrm{CH}\left(\mathrm{CH}_{3}\right)_{2}, 6 \mathrm{H}\right), 0.67\left(\mathrm{t}, \mathrm{J}_{\mathrm{HH}}=7.9 \mathrm{~Hz}, \mathrm{SiCH}_{2} \mathrm{CH}_{3}\right.$, $3 \mathrm{H}), 0.42\left(\mathrm{q}, \mathrm{J}_{\mathrm{HH}}=7.8 \mathrm{~Hz}, \mathrm{SiCH}_{2} \mathrm{CH}_{3}, 2 \mathrm{H}\right), 0.04$ (s, $\left.\mathrm{SiMe}_{2}, 6 \mathrm{H}\right) .{ }^{29} \mathrm{Si}$ from H,Si-HMBC $(80 \mathrm{MHz}$, $400 \mathrm{MHz}, 1: 5 \mathrm{DFB}:$ Toluene- $\left.d_{8}\right) \delta 37.9$.

[EtMe $\left.{ }_{2} \mathrm{SiNCCH}_{3}\right]\left[\mathrm{B}\left(\mathbf{C}_{6} \mathbf{F}_{5}\right)_{4}\right](\mathbf{2 1}) .{ }^{1} \mathrm{H}$ NMR $\left(400 \mathrm{MHz}, 1: 5 \mathrm{DFB}:\right.$ Toluene- $\left.d_{8}\right) \delta 1.36$ (s, NCMe, 3H), $0.67\left(\mathrm{t}, \mathrm{J}_{\mathrm{HH}}=7.9 \mathrm{~Hz}, \mathrm{SiCH}_{2} \mathrm{CH}_{3}, 3 \mathrm{H}\right.$ ), 0.41 (q, $\left.\mathrm{J}_{\mathrm{HH}}=8.0 \mathrm{~Hz}, \mathrm{SiCH}_{2} \mathrm{CH}_{3}, 2 \mathrm{H}\right), 0.03\left(\mathrm{~s}, \mathrm{SiMe}_{2}, 6 \mathrm{H}\right)$.

${ }^{29} \mathrm{Si}$ from H,Si-HMBC (80 MHz, $400 \mathrm{MHz}, 1: 5 \mathrm{DFB}:$ Toluene-d $) \delta 39.7$.

General Procedure for Method $\boldsymbol{b}$. A solution of in situ prepared ethyldimethylsilylnitrilium ion (0.026 mmol) in $100 \mu \mathrm{L}$ DFB and $200 \mu \mathrm{L}$ toluene- $d_{8}$ was added to a J. Young NMR tube and frozen in a liquid nitrogen cooled cold well before adding a solution of $\mathrm{Pd}(\mathrm{PCy})_{2}(17.3 \mathrm{mg}, 0.0260$ $\mathrm{mmol}$ ) in $300 \mu \mathrm{L}$ toluene- $d_{8}$. The reaction mixture was warmed to and mixed at $-78{ }^{\circ} \mathrm{C}$ before placing into the probe of a spectrometer pre-cooled to $-30^{\circ} \mathrm{C}$.

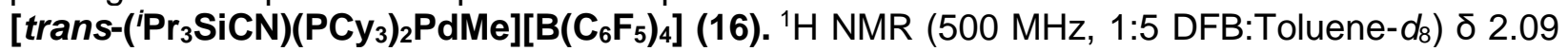
(m, partially obscured by solvent, Cy, $6 \mathrm{H}), 1.78(\mathrm{~m}, \mathrm{Cy}, 12 \mathrm{H}), 1.72(\mathrm{~d}, J=7.8 \mathrm{~Hz}, \mathrm{Cy} 12 \mathrm{H}), 1.66$ $(\mathrm{m}, 6 \mathrm{H}), 1.40(\mathrm{q}, J=11.0 \mathrm{~Hz}, 12 \mathrm{H}), 1.18-1.07\left(\mathrm{~m}, \mathrm{Cy}+\mathrm{CH}\left(\mathrm{CH}_{3}\right)_{3}, 21 \mathrm{H}\right), 1.00\left(\mathrm{~d}, \mathrm{~J}_{\mathrm{HH}}=7.2 \mathrm{~Hz}\right.$, $\left.\mathrm{CH}\left(\mathrm{CH}_{3}\right)_{2} 18 \mathrm{H}\right), 0.30$ (t, J $\left.\mathrm{HP}_{2}=5.8 \mathrm{~Hz}, \mathrm{Pd}-\mathrm{Me}, 3 \mathrm{H}\right) \cdot{ }^{13} \mathrm{C}\left\{{ }^{1} \mathrm{H}\right\} \mathrm{NMR}\left(151 \mathrm{MHz}, 1: 5 \mathrm{DFB}: T o l u e n e-d_{8}\right)$ $\delta 166.8$ (t, $\left.J_{\mathrm{CP}}=13.6 \mathrm{~Hz}, \mathrm{CNSi}\right), 34.5$ (t, $\left.J_{\mathrm{CP}}=9.0 \mathrm{~Hz}, \mathrm{Cy}\right), 30.8(\mathrm{Cy}), 27.7$ (t, $\left.J_{\mathrm{CP}}=5.4 \mathrm{~Hz}, \mathrm{Cy}\right)$, $26.6(\mathrm{Cy}), 17.4$ ('Pr), 12.48 ('Pr), -1.0 (t, $\left.\mathrm{J}_{\mathrm{CP}}=3.3 \mathrm{~Hz}, \mathrm{Pd}-\mathrm{Me}\right) .{ }^{31} \mathrm{P}\left\{{ }^{1} \mathrm{H}\right\} \mathrm{NMR}(202 \mathrm{MHz}$, Toluene$\left.d_{8}\right) \delta 32.5$.

trans-[(PhCN)(PCy $\left.)_{2} \mathrm{PdMe}\right]\left[\mathrm{B}\left(\mathrm{C}_{6} \mathrm{~F}_{5}\right)_{4}\right](9)$. In a glovebox, $\mathrm{PCy}_{3}(16.8 \mathrm{mg}, 0.06 \mathrm{mmol})$ and (COD)PdMeCl $(8.0 \mathrm{mg}, 0.03 \mathrm{mmol}$ ) were combined in $600 \mu \mathrm{L}$ of dichloromethane. To this reaction solution, benzonitrile $(3.1 \mu \mathrm{L}, 0.03 \mathrm{mmol})$ was added. The reaction mixture was added to solid $\mathrm{Na}\left[\mathrm{B}\left(\mathrm{C}_{6} \mathrm{~F}_{5}\right)_{4}\right](21.1 \mathrm{mg}, 0.03 \mathrm{mmol})$. After mixing, the mixture was filtered through a $0.2 \mu \mathrm{m}$ PTFE syringe filter and the solvent was evacuated. The resulting residue was washed with hexanes (3 $x 1 \mathrm{~mL}$ ). The product was isolated as a beige film by drying under high vacuum. (42.3 $\mathrm{mg}, 0.029$, 96\%). ${ }^{1} \mathrm{H}$ NMR (400 MHz, $\mathrm{CD}_{2} \mathrm{Cl}_{2}$ ) $\delta 7.82(\mathrm{tt}, J=7.6,1.4 \mathrm{~Hz}, \mathrm{ArH}, 1 \mathrm{H}), 7.67(\mathrm{~m}, \mathrm{ArH}, 4 \mathrm{H}), 2.15$ (tt, $J=12.4,3.1 \mathrm{~Hz}, \mathrm{Cy}, 6 \mathrm{H}), 1.89(\mathrm{~m}, \mathrm{Cy}, 24 \mathrm{H}), 1.74(\mathrm{~m}, \mathrm{Cy}, 6 \mathrm{H}), 1.60(\mathrm{q}, J=12.1 \mathrm{~Hz}, \mathrm{Cy}, 12 \mathrm{H})$, $1.23(\mathrm{~m}, \mathrm{Cy}, 18 \mathrm{H}), 0.40(\mathrm{t}, J=5.6 \mathrm{~Hz}, \mathrm{Pd}-\mathrm{Me}, 3 \mathrm{H}) .{ }^{13} \mathrm{C}\left\{{ }^{1} \mathrm{H}\right\}$ NMR $\left(101 \mathrm{MHz}, \mathrm{CD}_{2} \mathrm{Cl}_{2}\right) \delta 136.0$, 132.5, 130.7, 123.3, 109.3, 32.9 (vt, $J=9.7 \mathrm{~Hz}, \mathrm{Cy}$ ), 30.4 (Cy), 28.1 (vt, $J=5.4 \mathrm{~Hz}, \mathrm{Cy}$ ), 26.7 (Cy), -4.96 (t, $J=3.5 \mathrm{~Hz}, \mathrm{Pd}-\mathrm{Me}) .{ }^{31} \mathrm{P}\left\{{ }^{1} \mathrm{H}\right\} \mathrm{NMR}\left(162 \mathrm{MHz}, \mathrm{CD}_{2} \mathrm{Cl}_{2}\right) \delta 28.1$. 
General Procedure for Silylium Transfer Between Hexyl Methyl Ether and Nitrile. To a solution of in situ generated and room temperature ethyldimethylsilylnitrilium $(0.026 \mathrm{mmol})$, was added hexyl methyl ether $(3.9 \mu \mathrm{L}, 0.026 \mathrm{mmol})$.

[(Hexyl)(methyl)(ethyldimethylsilyloxonium)][B( $\left.\left.\mathbf{C}_{6} \mathbf{F}_{5}\right)_{4}\right] \quad(22)$. Ethyldimethylsilane $(4.1 \mu \mathrm{L}$, $0.031 \mathrm{mmol})$ was added to a room temperature solution of $\left[\mathrm{Ph}_{3} \mathrm{C}\right]\left[\mathrm{B}\left(\mathrm{C}_{6} \mathrm{~F}_{5}\right)_{4}\right](24 \mathrm{mg}, 0.026 \mathrm{mmol})$ and diphenyl ether $(4.1 \mu \mathrm{L}, 0.026 \mathrm{mmol})$ in $100 \mu \mathrm{L}$ DFB and $500 \mu \mathrm{L}$ toluene- $d_{8}$. The solution changed color from bright orange to colorless, indicative of silyloxonium ion generation. Hexyl methyl ether $(3.9 \mu \mathrm{L}, 0.026 \mathrm{mmol})$ was added to the solution of in situ generated diphenylsilyloxonium ion to produce the product. ${ }^{1} \mathrm{H}$ NMR $\left(600 \mathrm{MHz}, 1: 5 \mathrm{DFB}\right.$ :Toluene- $\left.d_{8}\right) \delta 3.52$ $(\mathrm{m}, 2 \mathrm{H}), 3.08(\mathrm{~s}, 3 \mathrm{H}), 1.23\left(\mathrm{p}, \mathrm{J}_{\mathrm{HH}}=7.7 \mathrm{~Hz}, 2 \mathrm{H}\right), 1.16(\mathrm{~m}, 2 \mathrm{H}), 1.03(\mathrm{~m}, 2 \mathrm{H}), 0.85(\mathrm{~m}, 5 \mathrm{H}), 0.60$ $\left(\mathrm{t}, J_{\mathrm{HH}}=8.0 \mathrm{~Hz}, \mathrm{SiCH}_{2} \mathrm{CH}_{3}, 3 \mathrm{H}\right), 0.32\left(\mathrm{q}, \mathrm{J}_{\mathrm{HH}}=8.0 \mathrm{~Hz}, \mathrm{SiCH}_{2} \mathrm{CH}_{3}, 2 \mathrm{H}\right),-0.06\left(\mathrm{~s}, \mathrm{SiMe}_{2}, 6 \mathrm{H}\right) .{ }^{13} \mathrm{C}\left\{{ }^{1} \mathrm{H}\right\}$ NMR (151 MHz, 1:5 DFB:Toluene-d $\left.d_{8}\right) \delta 84.1\left(\mathrm{OCH}_{2}\right), 63.5(\mathrm{OMe}), 31.3,26.6,24.1,22.7,13.8$, $6.3\left(\mathrm{SiCH}_{2} \mathrm{CH}_{3}\right), 5.0\left(\mathrm{SiCH}_{2} \mathrm{CH}_{3}\right),-4.3 \quad\left(\mathrm{SiMe}_{2}\right) .{ }^{29} \mathrm{Si}, \mathrm{H}-\mathrm{HMBC}(100 \mathrm{MHz}, 500 \mathrm{MHz}, 1: 5$ DFB:Toluene- $\left.d_{8}\right) \delta 72.2$. 


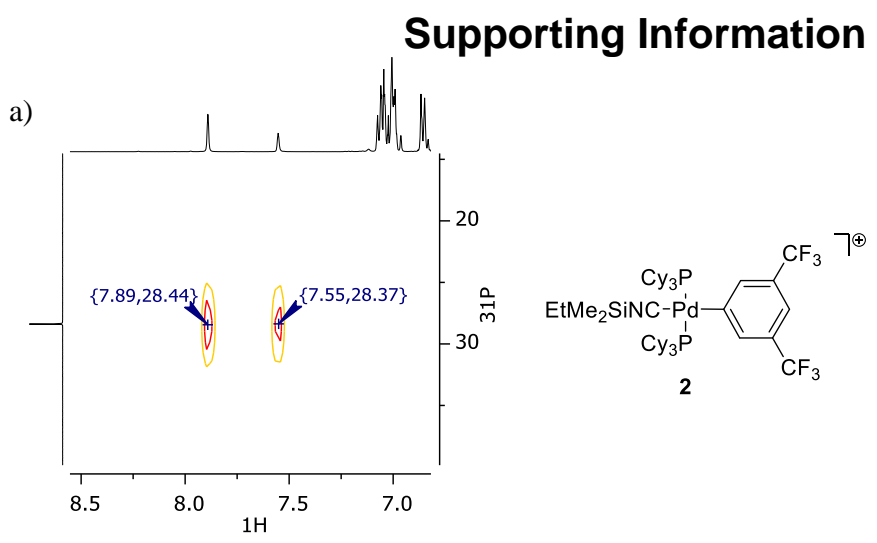

b)

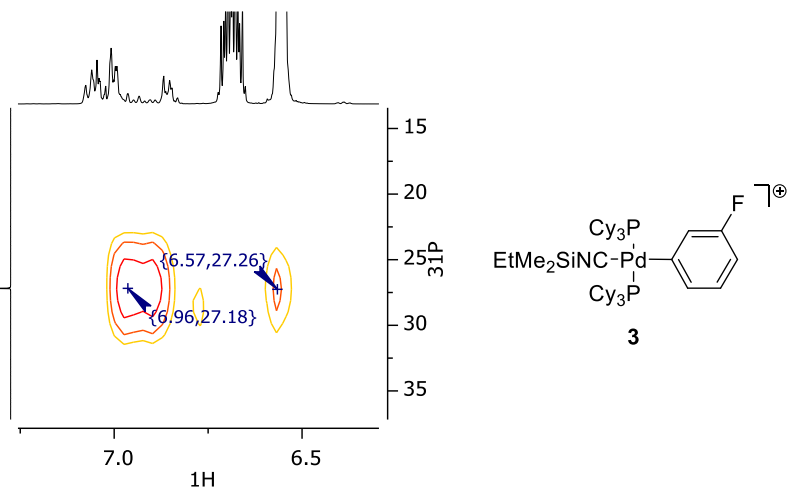

c)

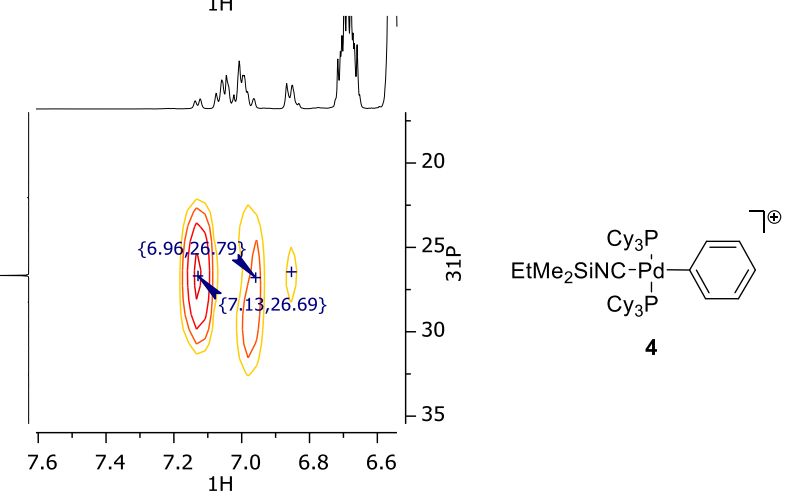

Figure S1: Selected room temperature ${ }^{1} \mathrm{H},{ }^{31} \mathrm{P}-\mathrm{HMBC}(500 \mathrm{MHz}, 202 \mathrm{MHz})$ spectra for a) 2, b) 3, and c) 4, highlighting the coupling between the aromatic proton and phosphorus resonances. Note that some aromatic proton resonances are obscured by solvent. Solvent: 1:5 DFB:Tol- $d_{8}$. 
a)

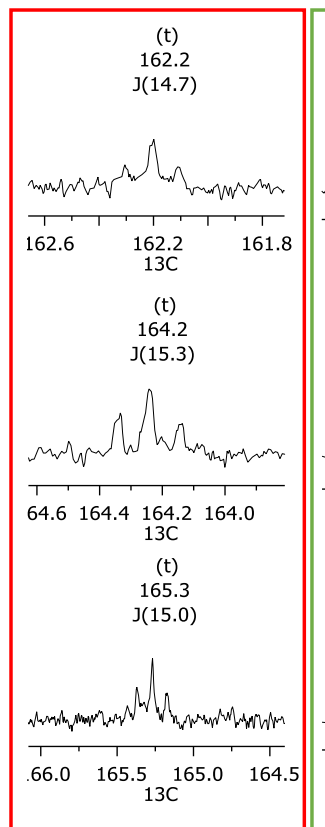

b)

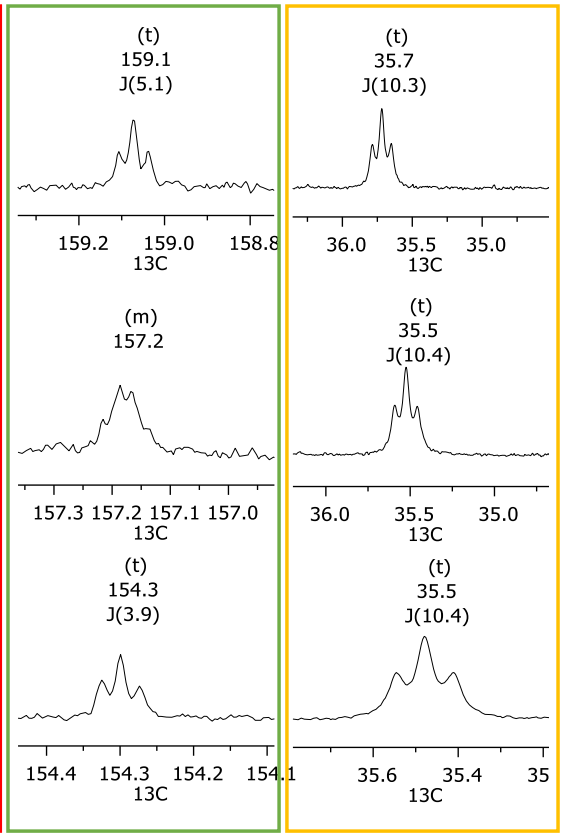

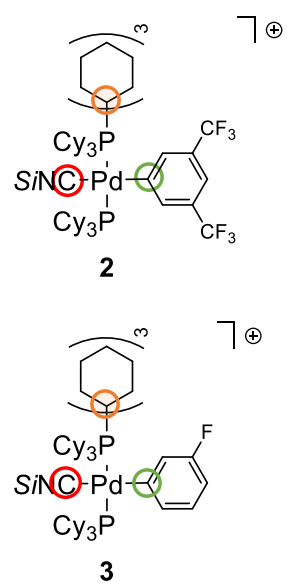

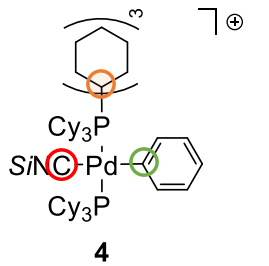

Figure S2: Selected room temperature $151 \mathrm{MHz}{ }^{13} \mathrm{C}\left\{{ }^{1} \mathrm{H}\right\}$ NMR spectra for products 2-4, highlighting the multiplicity of the (a) isocyano carbon, (b) ipso aryl carbon, and (c) the $\mathrm{PCy}_{3}$ methyne carbon resonances. The splitting pattern of the ipso aryl carbon atom of $\mathbf{3}$ is complicated by additional coupling to fluorine. Solvent: 1:5 DFB:Tol- $d_{8}$. Si $=\mathrm{SiMe}_{2} \mathrm{Et}$. 


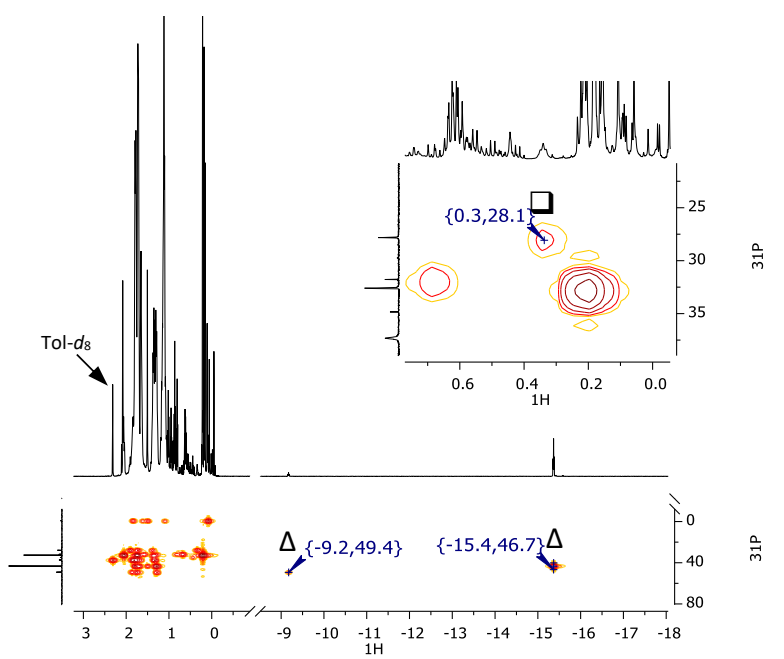

Figure S3: ${ }^{1} \mathrm{H}, \mathrm{P}-\mathrm{HMBC}(500 \mathrm{MHz}, 202 \mathrm{MHz})$ spectra of a solution of in situ generated 6 after 1 day at room temperatures, highlighting decomposition to palladium-hydrides $(\Delta)$ and an apparent Pd-Me complex ( $\square)$. Solvent: 1:5 DFB-Tol- $d_{8}$.

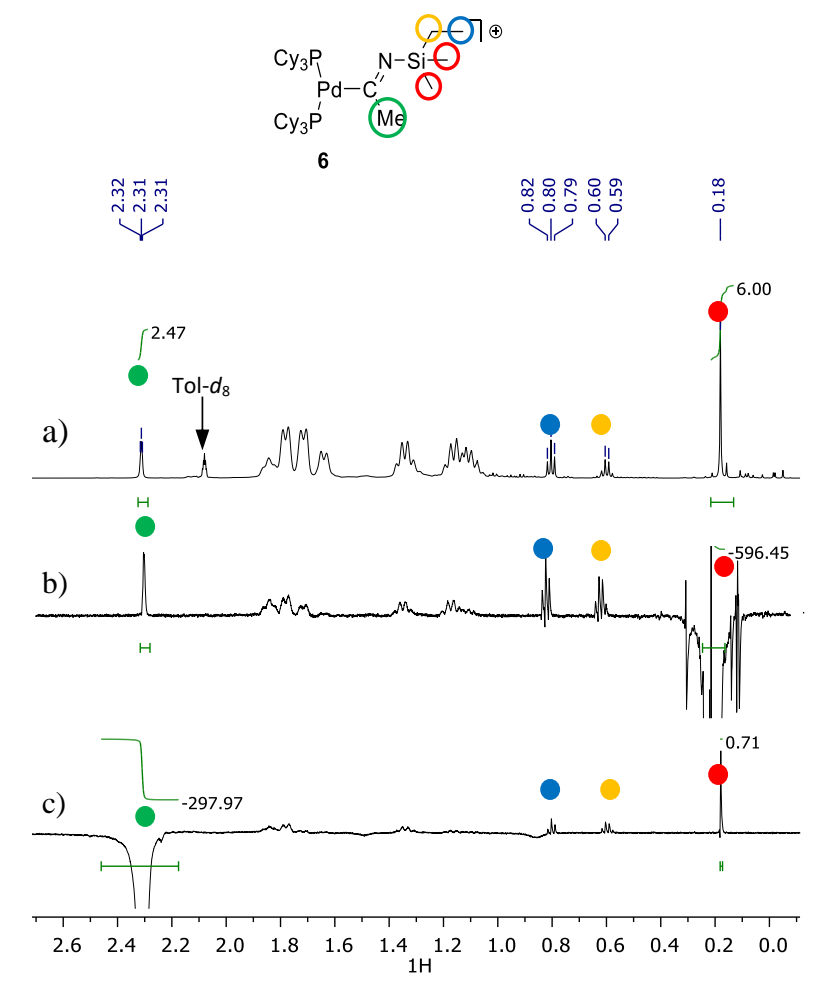

Figure S4: 1-D NOESY experiments applied to a room temperature solution of in situ generated $6\left(t_{\text {mix }}=300 \mathrm{~ms}\right)$, highlighting the proximity of the silyl moiety to the iminoacyl methyl group. a) Normal $500 \mathrm{MHz}{ }^{1} \mathrm{H}$ NMR spectrum. b) With selective excitation of the $-\mathrm{SiMe}_{2} \mathrm{Et}$ resonances at 0.23 ppm. c) With selective excitation of the $-\mathrm{C}=(\mathrm{NSi}) \mathrm{CH}_{3}$ resonances at $2.36 \mathrm{ppm}$. Solvent: 1:5 DFB:Tol-d. $S i=$ SiMe $_{2}$ Et. 


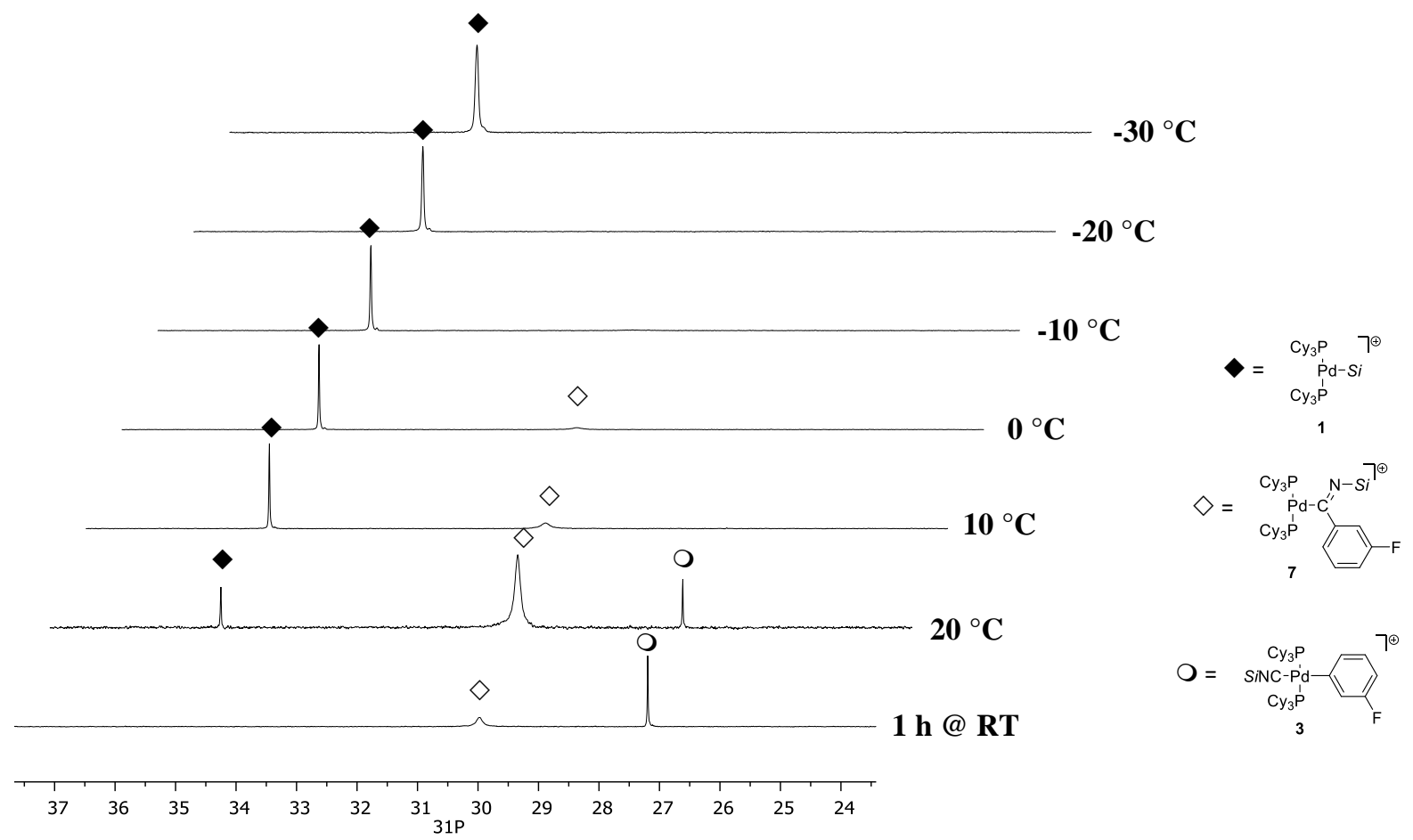

Figure S5: Selected $202 \mathrm{MHz}$ variable-temperature ${ }^{31} \mathrm{P}\left\{{ }^{1} \mathrm{H}\right\}$ NMR spectra for the reaction which generated $\mathbf{7}$ by method $a$, emphasizing the conversion of $\mathbf{1} \rightarrow \mathbf{7}$ and $\mathbf{7} \rightarrow \mathbf{3}$ upon warming. Solvent: 1:5 DFB:Tol- $d_{8} . \diamond=\mathbf{1}, \diamond=\mathbf{7}, \bigcirc=\mathbf{3}$.

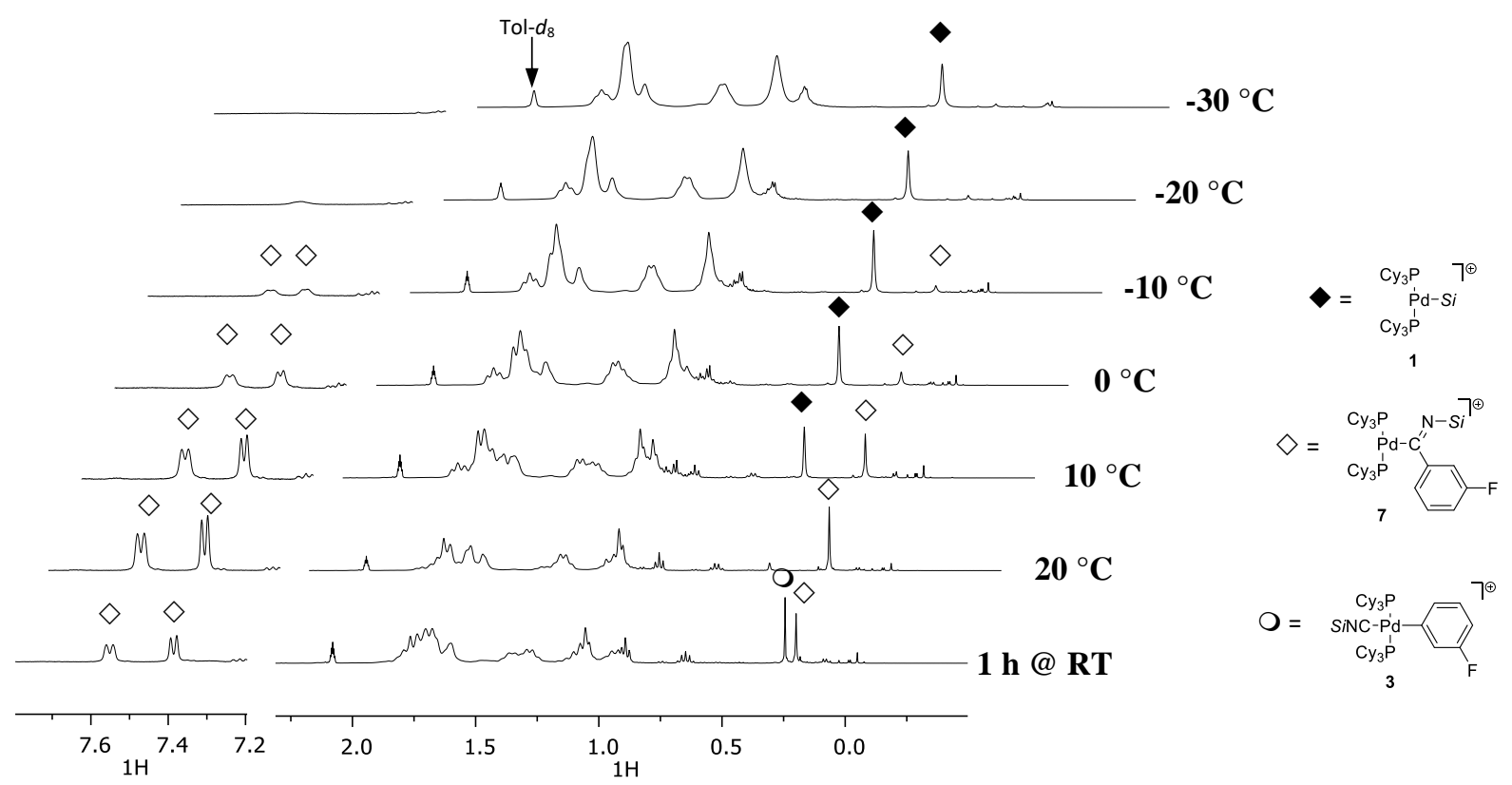

Figure S6: Selected $400 \mathrm{MHz}$ variable temperature ${ }^{1} \mathrm{H}$ NMR spectra for the reaction which generated $\mathbf{7}$ by method $a$, emphasizing the conversion of $\mathbf{1} \rightarrow \mathbf{7}$ and $\mathbf{7} \rightarrow \mathbf{3}$ upon warming. The aromatic and $-\mathrm{SiMe}_{2} \mathrm{Et}$ protons are noted. The aromatic proton resonances for $\mathbf{3}$ are upfield those of 7 and are obscured by solvent. Solvent: 1:5 DFB:Tol- $d_{8} . \diamond=\mathbf{1}, \diamond=\mathbf{7}, \mathrm{O}=\mathbf{3}$. 

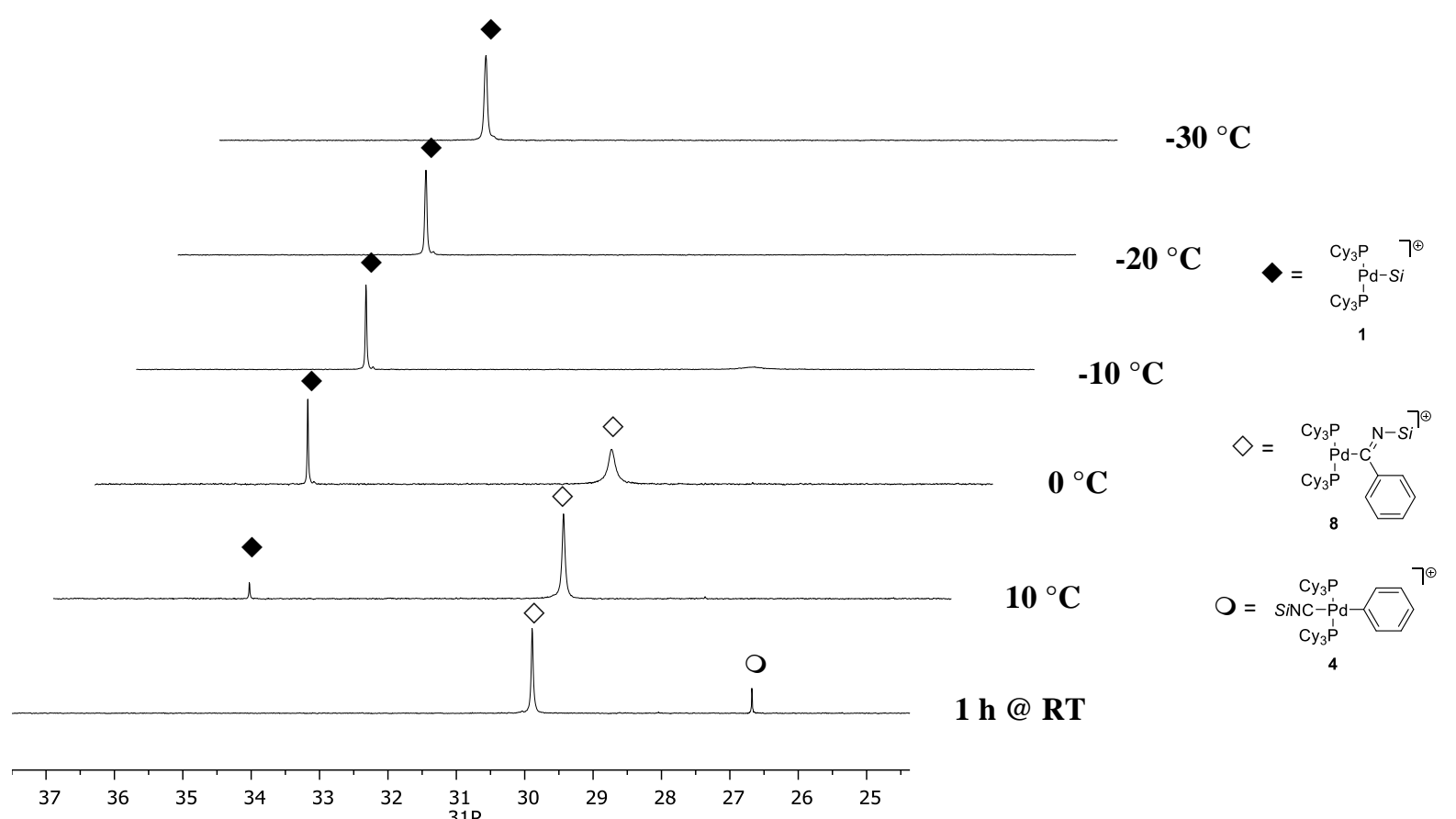

Figure S7: Selected $202 \mathrm{MHz}$ variable-temperature ${ }^{31} \mathrm{P}\left\{{ }^{1} \mathrm{H}\right\}$ NMR spectra for the reaction which generated $\mathbf{8}$ by method $a$, emphasizing the conversion of $\mathbf{1} \rightarrow \mathbf{8}$ and $\mathbf{8} \rightarrow \mathbf{4}$ upon warming. Solvent: 1:5 DFB:Tol-d $8 . \diamond=\mathbf{1}, \diamond=\mathbf{8}, \bigcirc=\mathbf{4}$.

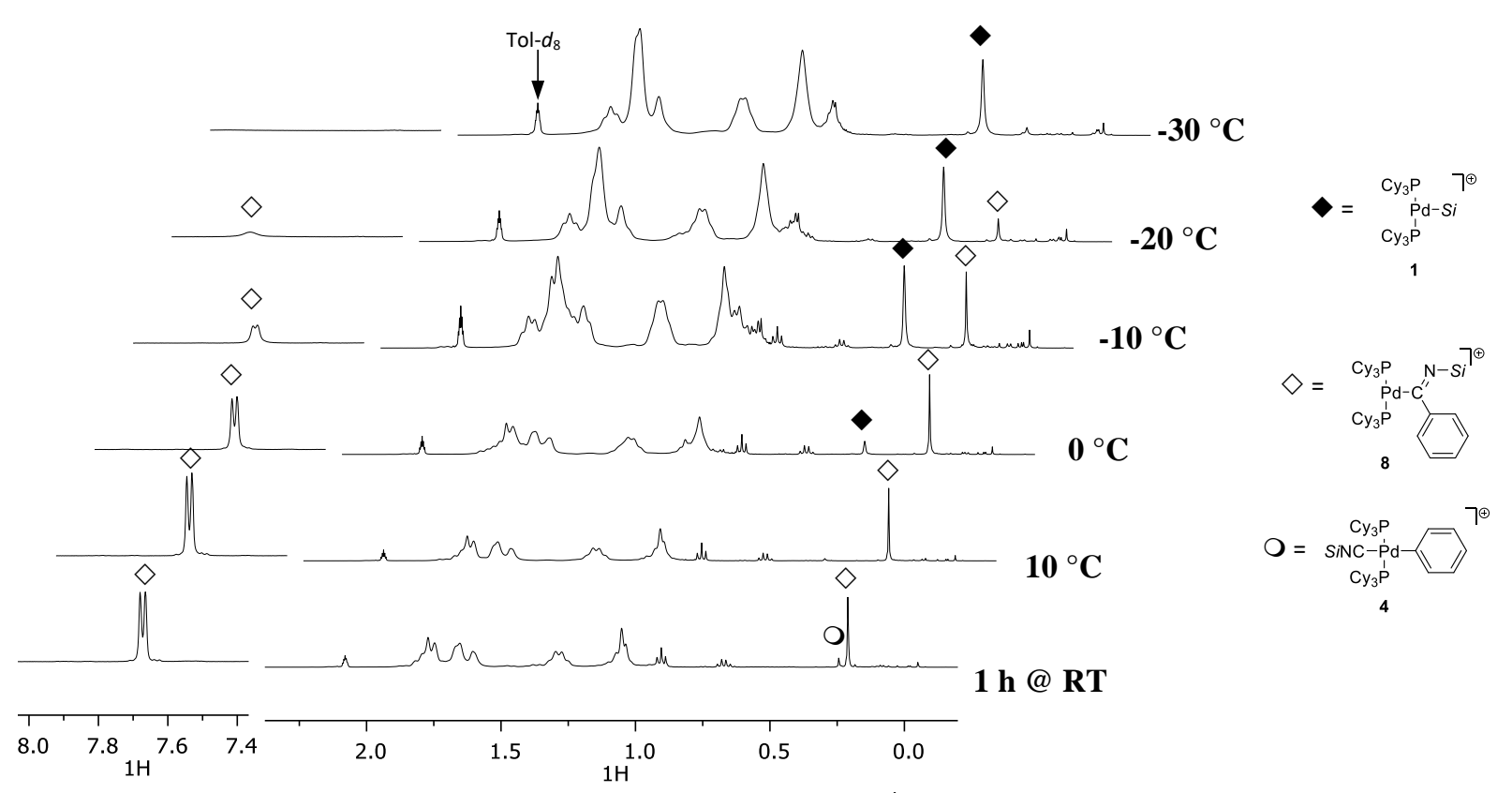

Figure S8: Selected $400 \mathrm{MHz}$ variable temperature ${ }^{1} \mathrm{H}$ NMR spectra for the reaction which generated $\mathbf{8}$ following method $a$, emphasizing the conversion of $\mathbf{1} \rightarrow \mathbf{8}$ and $\mathbf{8} \rightarrow \mathbf{4}$ upon warming. The aromatic and $-\mathrm{SiMe}_{2} \mathrm{Et}$ protons are noted. The aromatic proton resonances for $\mathbf{4}$ are weak, due to its low concentration in this spectrum, and obscured. Solvent: 1:5 DFB:Tol- $d_{8} . \diamond=\mathbf{1}, \diamond=$ 9, $\mathrm{O}=4$. 


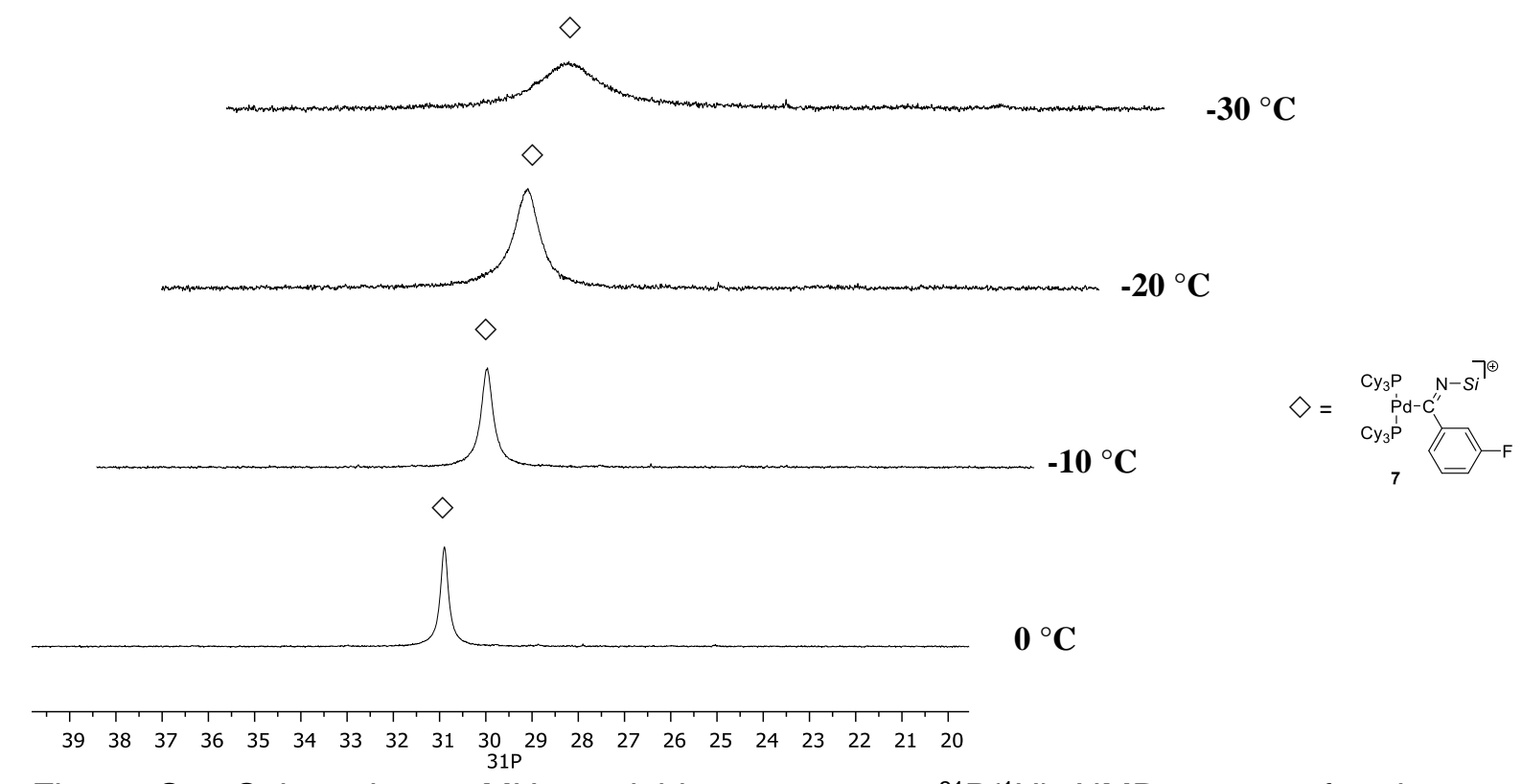

Figure S9: Selected $202 \mathrm{MHz}$ variable-temperature ${ }^{31} \mathrm{P}\left\{{ }^{1} \mathrm{H}\right\}$ NMR spectra for the reaction generating 7 by method $b$, emphasizing 7 as the major product at $-30{ }^{\circ} \mathrm{C}$. Solvent: 1:5 DFB:Tol$d_{8} \diamond=7$.

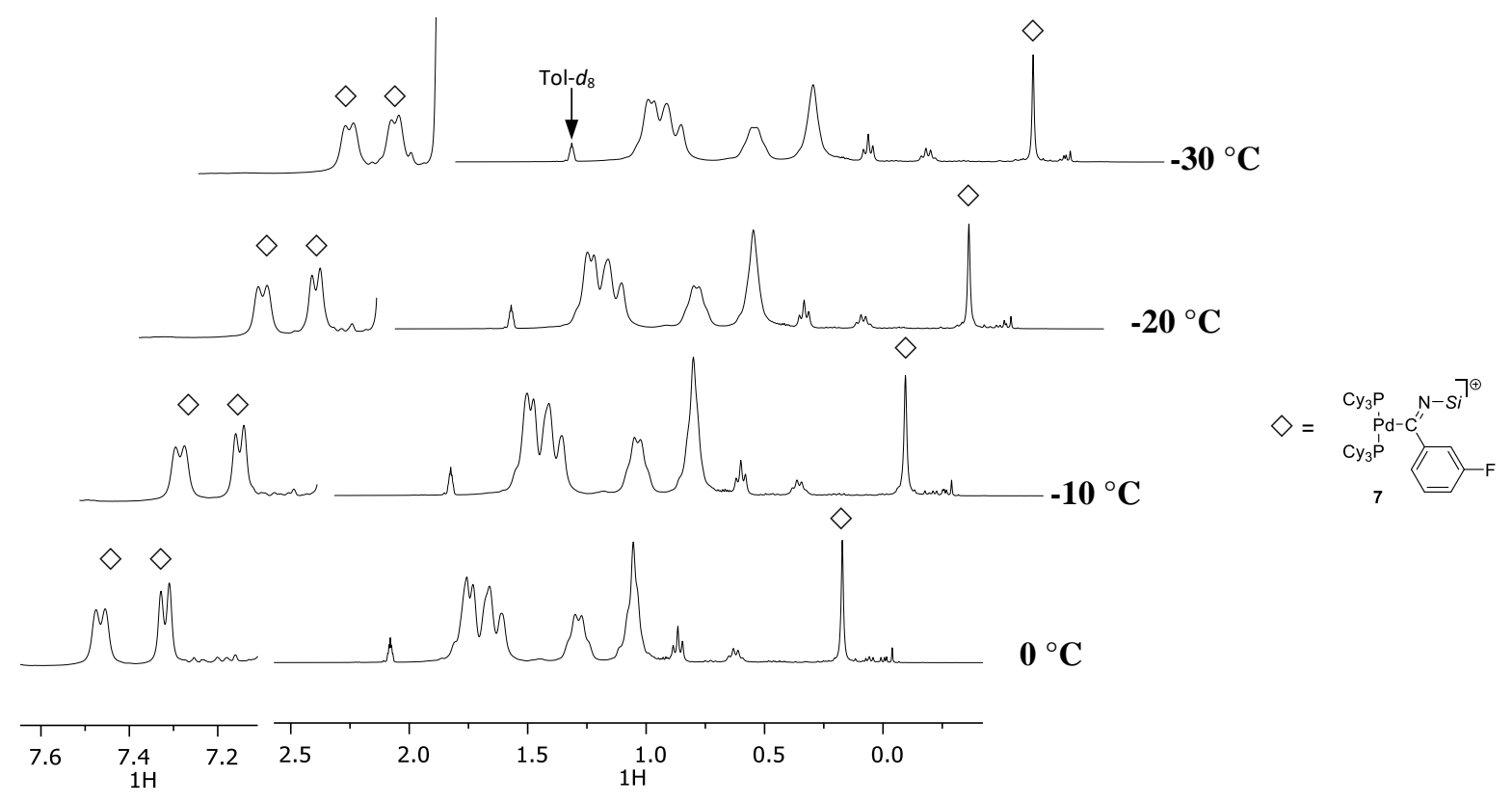

Figure S10: Selected $400 \mathrm{MHz}$ variable temperature ${ }^{1} \mathrm{H}$ NMR spectra for the reaction generating 7 by method $b$, emphasizing 7 as the major product at $-30^{\circ} \mathrm{C}$. The aromatic and $-\mathrm{SiMe}$ Et protons are noted. Solvent: 1:5 DFB:Tol- $d_{8} . \diamond=7$. 


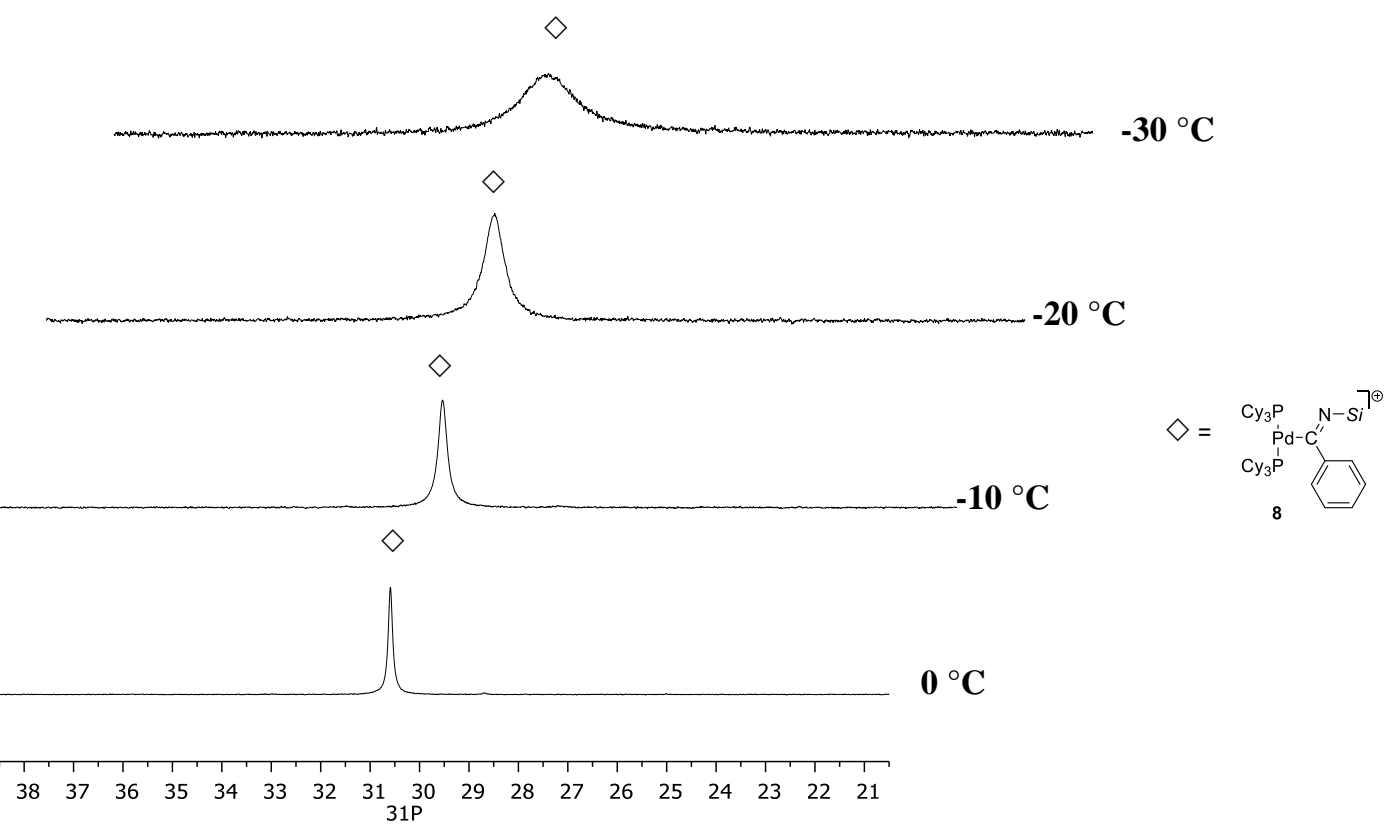

Figure S11: Selected $202 \mathrm{MHz}$ variable-temperature ${ }^{31} \mathrm{P}\left\{{ }^{1} \mathrm{H}\right\}$ NMR spectra for the reaction which generated 8 by method $b$, emphasizing 8 as the major product at $-30^{\circ} \mathrm{C}$. Solvent: 1:5 DFB:Tol$d_{8 .} \diamond=8$.

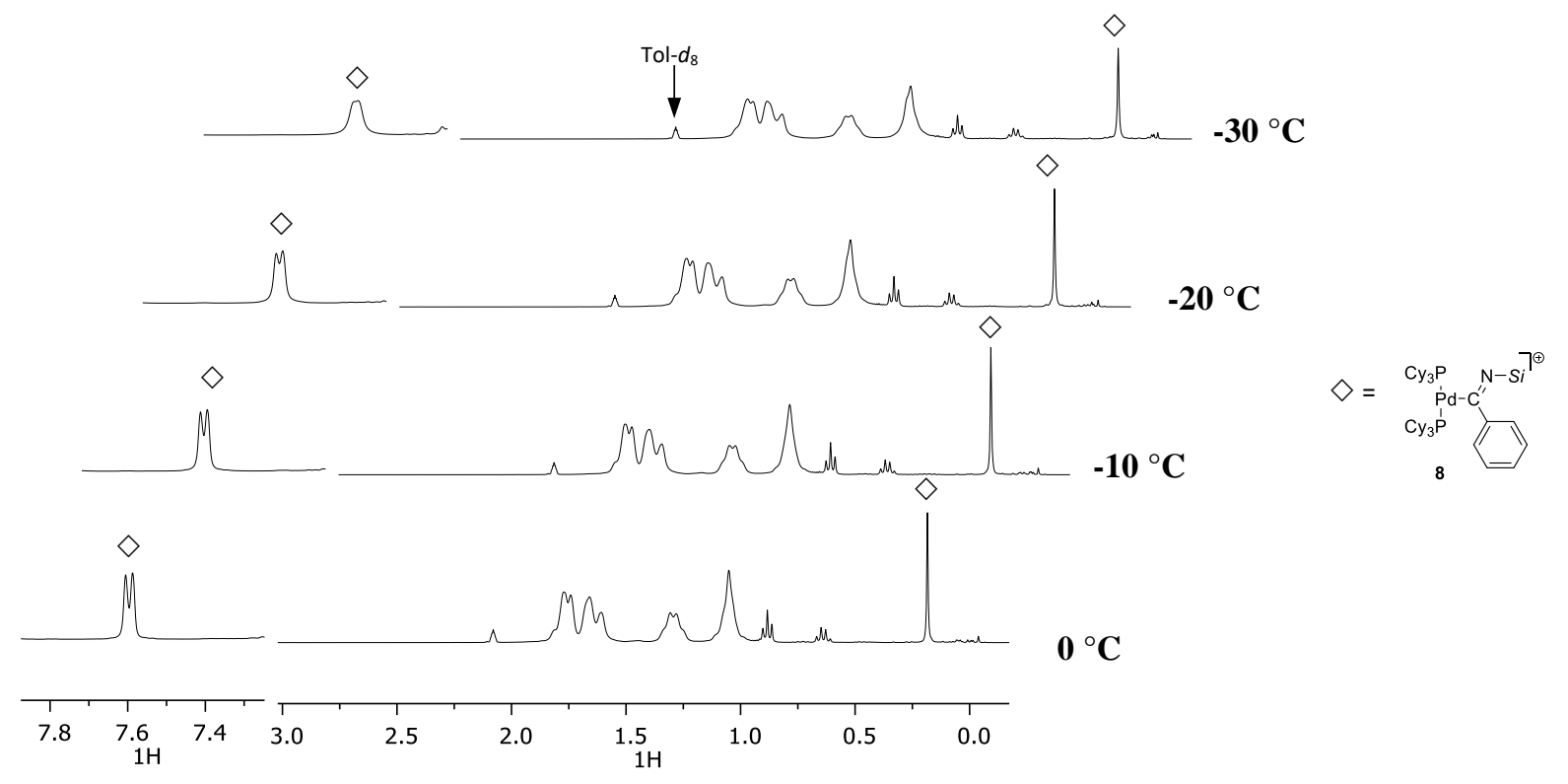

Figure S12: Selected $400 \mathrm{MHz}$ variable temperature ${ }^{1} \mathrm{H}$ NMR spectra for the reaction which generated 8 following by $b$, emphasizing 8 as the major product at $-30^{\circ} \mathrm{C}$. The aromatic and $\mathrm{SiMe}_{2}$ Et protons are noted. Solvent: 1:5 DFB:Tol- $d_{8} . \diamond=\mathbf{8}$. 


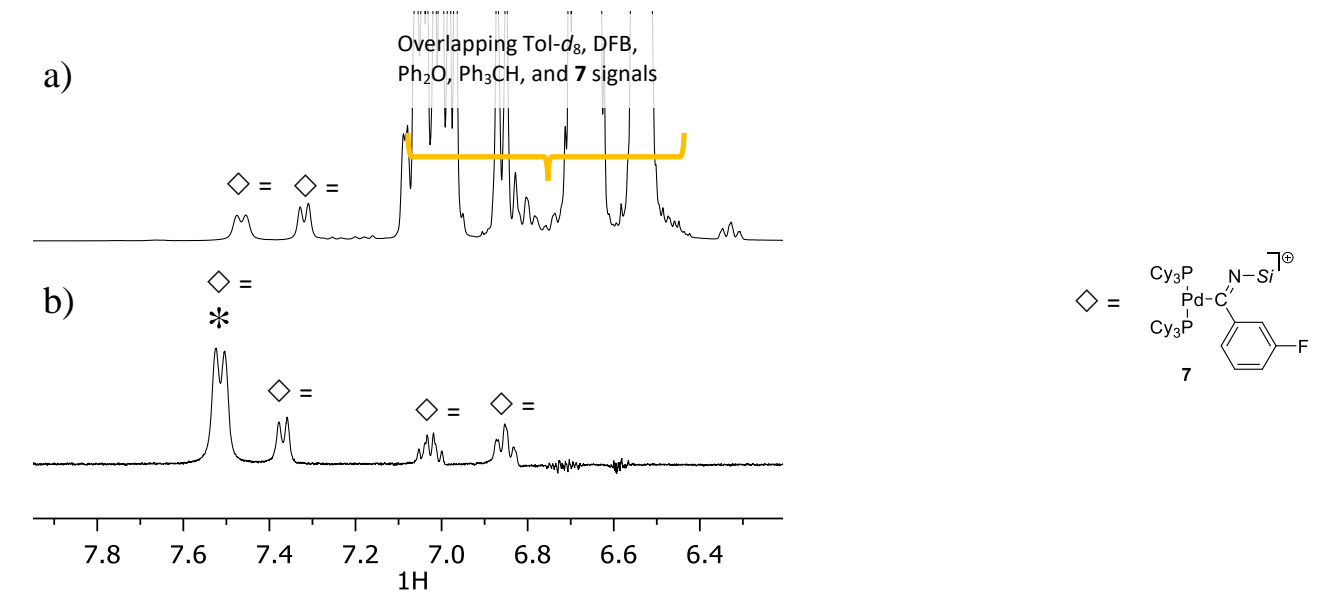

Figure S13: Selective 1-D TOCSY experiment applied to a solution containing 7 at $0{ }^{\circ} \mathrm{C}$, highlighting the obscured aromatic proton resonances. a) Normal $400 \mathrm{MHz}{ }^{1} \mathrm{H}$ NMR spectrum. b) With selective excitation at $7.52 \mathrm{ppm}^{*}\left(\mathrm{t}_{\text {mix }}=80 \mathrm{~ms}\right)$. Solvent: in 1:5 DFB:Tol- $d_{8}$. Temperature: 0 ${ }^{\circ} \mathrm{C}$.

a) Overlapping Tol- $d_{8}$, DFB,

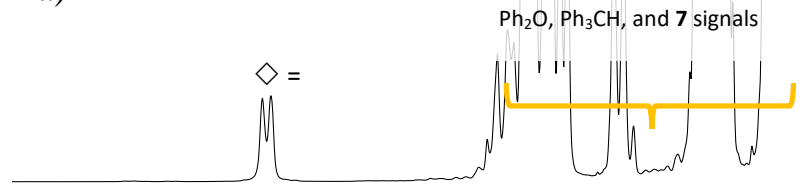

b)
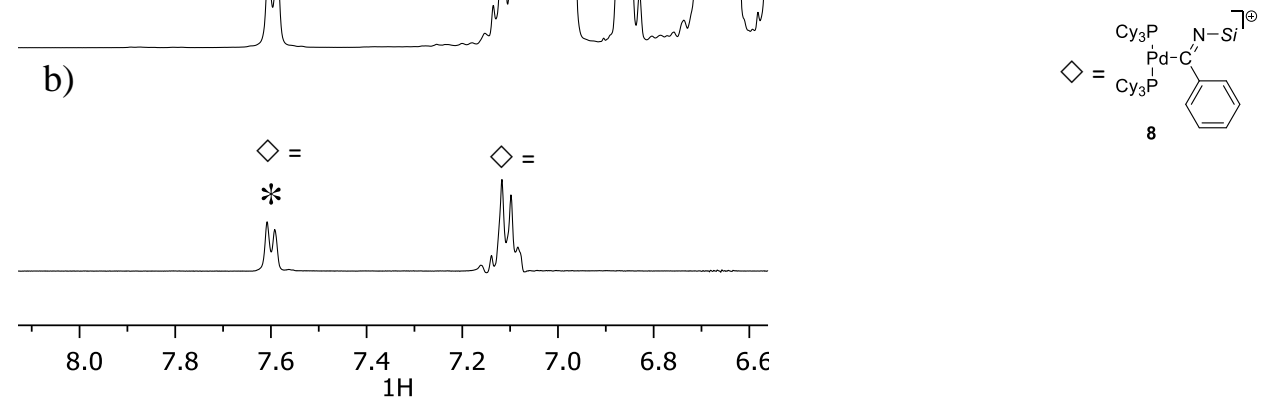

Figure S14: 1-D TOCSY experiments applied to a solution containing 8 at $0{ }^{\circ} \mathrm{C}$, highlighting the obscured aromatic proton resonances. a) Normal $400 \mathrm{MHz}{ }^{1} \mathrm{H}$ NMR spectrum. b) With selective excitation at $7.60 \mathrm{ppm}^{*}\left(\mathrm{t}_{\mathrm{mix}}=80 \mathrm{~ms}\right)$. Solvent: 1:5 DFB:Tol- $d_{8}$. Temperature: $0{ }^{\circ} \mathrm{C}$. 


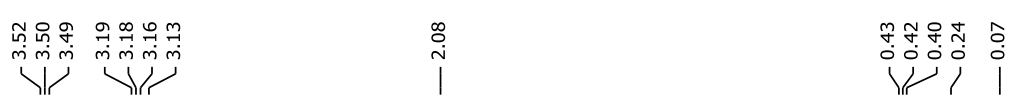

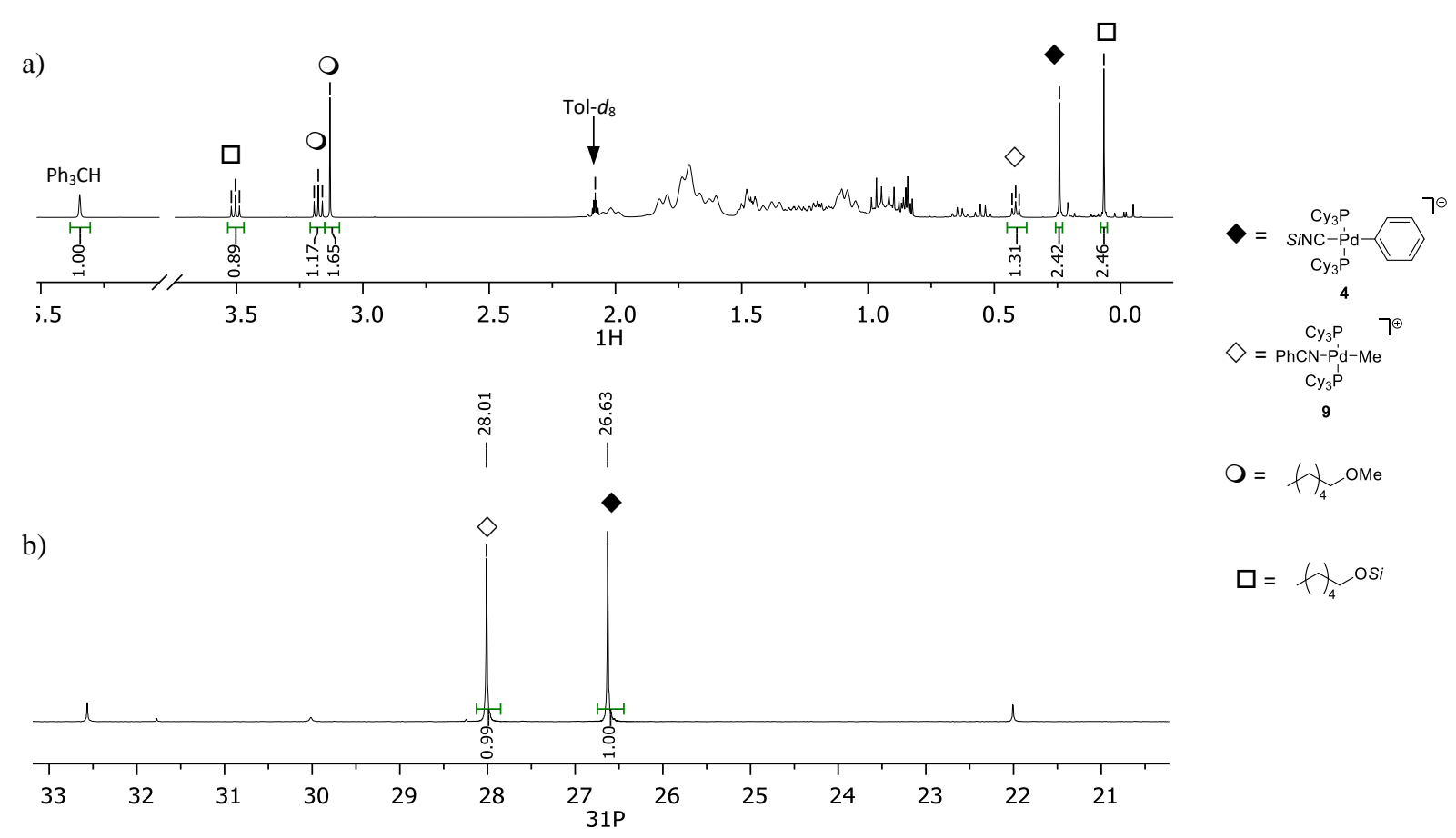

Figure S15: Selected NMR spectra for the demethylation of hexyl methyl ether by $\mathbf{8}$, after 1 day at ambient temperatures. a) $400 \mathrm{MHz}{ }^{1} \mathrm{H}$ NMR spectrum. b) $162 \mathrm{MHz}{ }^{31} \mathrm{P}\left\{{ }^{1} \mathrm{H}\right\}$ NMR spectrum. Solvent: 1:5 DFB:Tol- $d_{8} . \diamond=\mathbf{4}, \diamond=\mathbf{9}, \mathrm{O}=$ hexyl methyl ether, $\square=\mathrm{CH}_{3}\left(\mathrm{CH}_{2}\right)_{4} \mathrm{CH}_{2} \mathrm{OSiMe}{ }_{2} \mathrm{Et}$. 


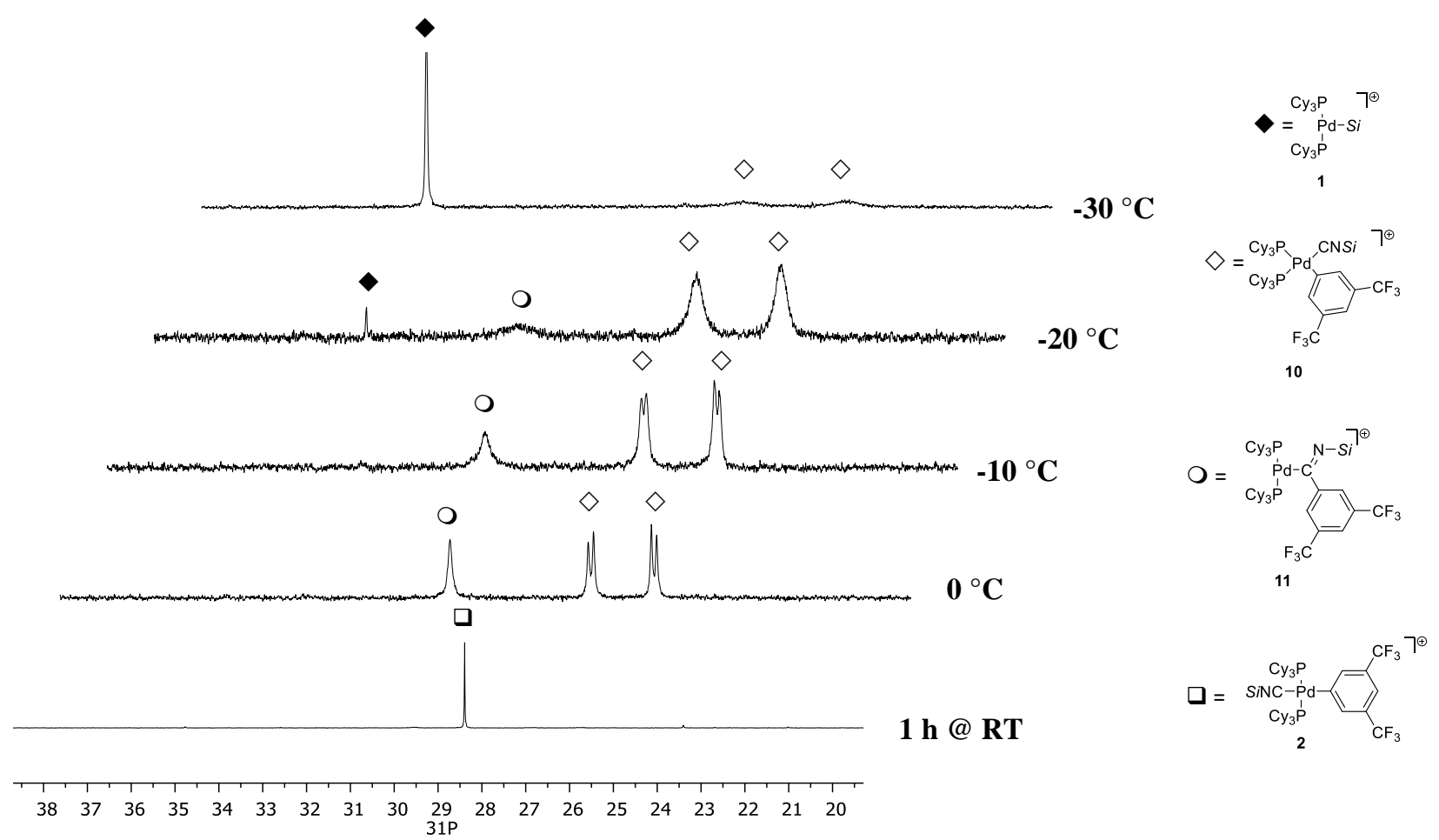

Figure S16: Selected $202 \mathrm{MHz}$ variable-temperature ${ }^{31} \mathrm{P}\left\{{ }^{1} \mathrm{H}\right\}$ NMR spectra for the reaction which generated 10 and 11 by method a, emphasizing the conversion of $1 \rightarrow 10 \& 11$ and $10 \& 11 \rightarrow 2$ upon warming. Solvent: 1:5 DFB:Tol- $d_{8} . \bullet=\mathbf{1}, \diamond=\mathbf{1 0}, O=\mathbf{1 1}, \square=\mathbf{2}$.

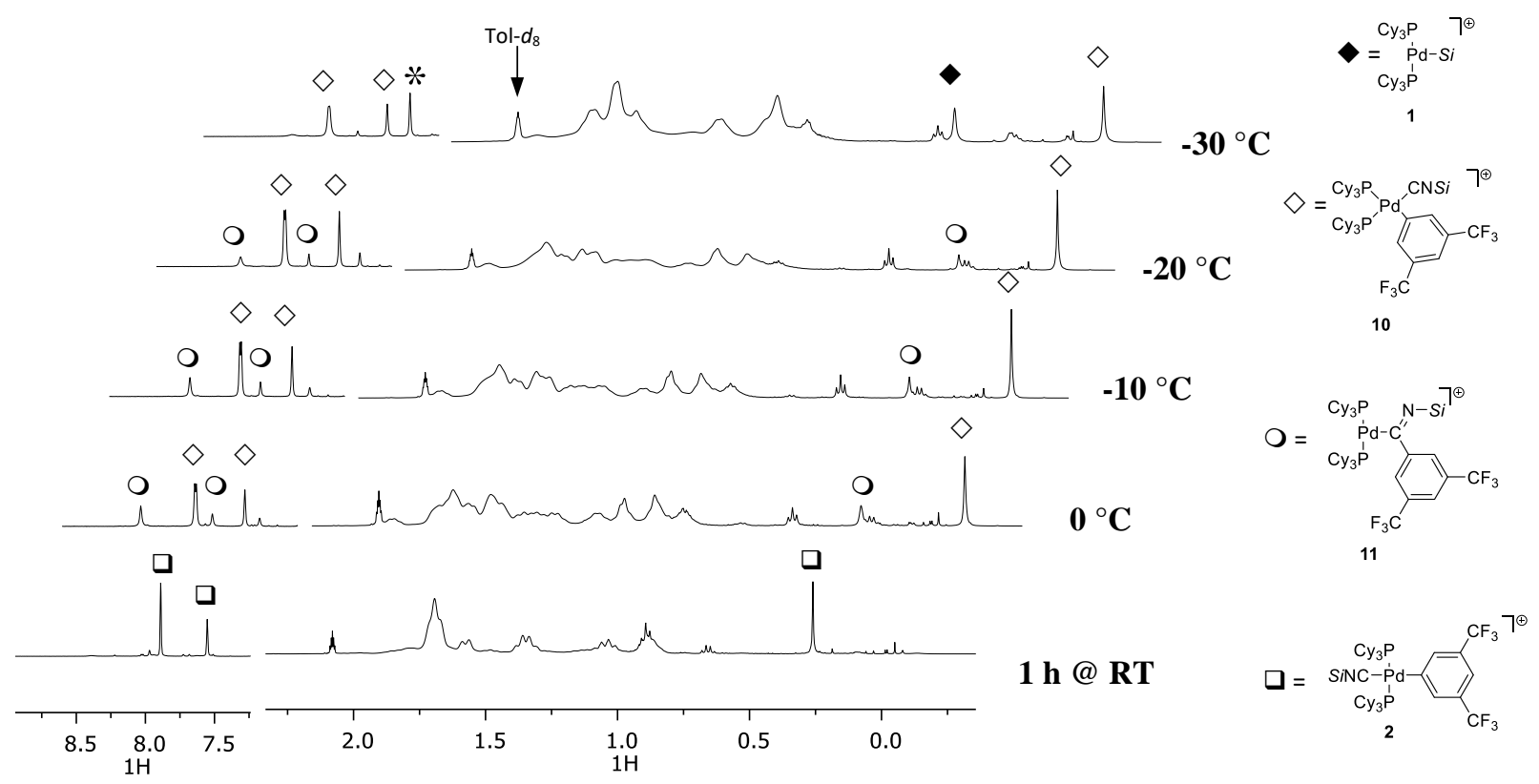

Figure S17: Selected $400 \mathrm{MHz}$ variable temperature ${ }^{1} \mathrm{H}$ NMR spectra for the reaction which generated 10 and 11 by method a, emphasizing the conversion of $1 \rightarrow 10 \& 11$ and $10 \& 11 \rightarrow 2$ upon warming. The aromatic and $-\mathrm{SiMe}_{2}$ Et protons are noted. Solvent: 1:5 DFB:Tol- $d_{8} . \diamond=\mathbf{1}, \diamond$ $=10, \mathrm{O}=11, \square=2,{ }^{*}=\mathrm{NCArF}_{6}$. 


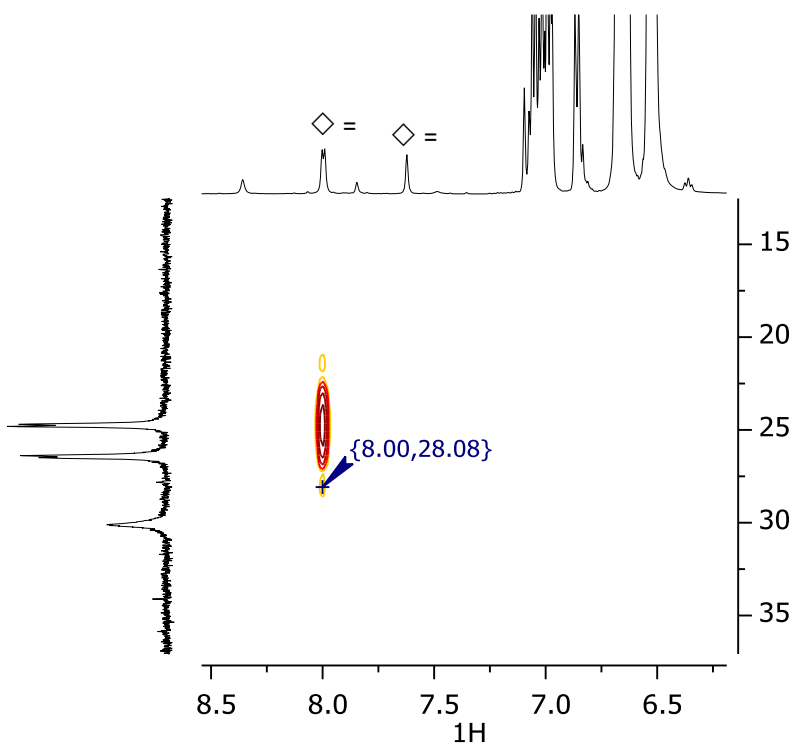

$\stackrel{\circ}{m}$

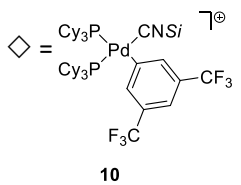

Figure S18: H,P-HMBC (500 MHz, $202 \mathrm{MHz}$ ) spectra of a solution of in situ generated 10 at -10 ${ }^{\circ} \mathrm{C}$, highlighting the coupling between an ortho $\mathrm{ArF}_{6}$ proton and the trans phosphorus atom. Solvent: 1:5 DFB-Tol- $d_{8}$. 


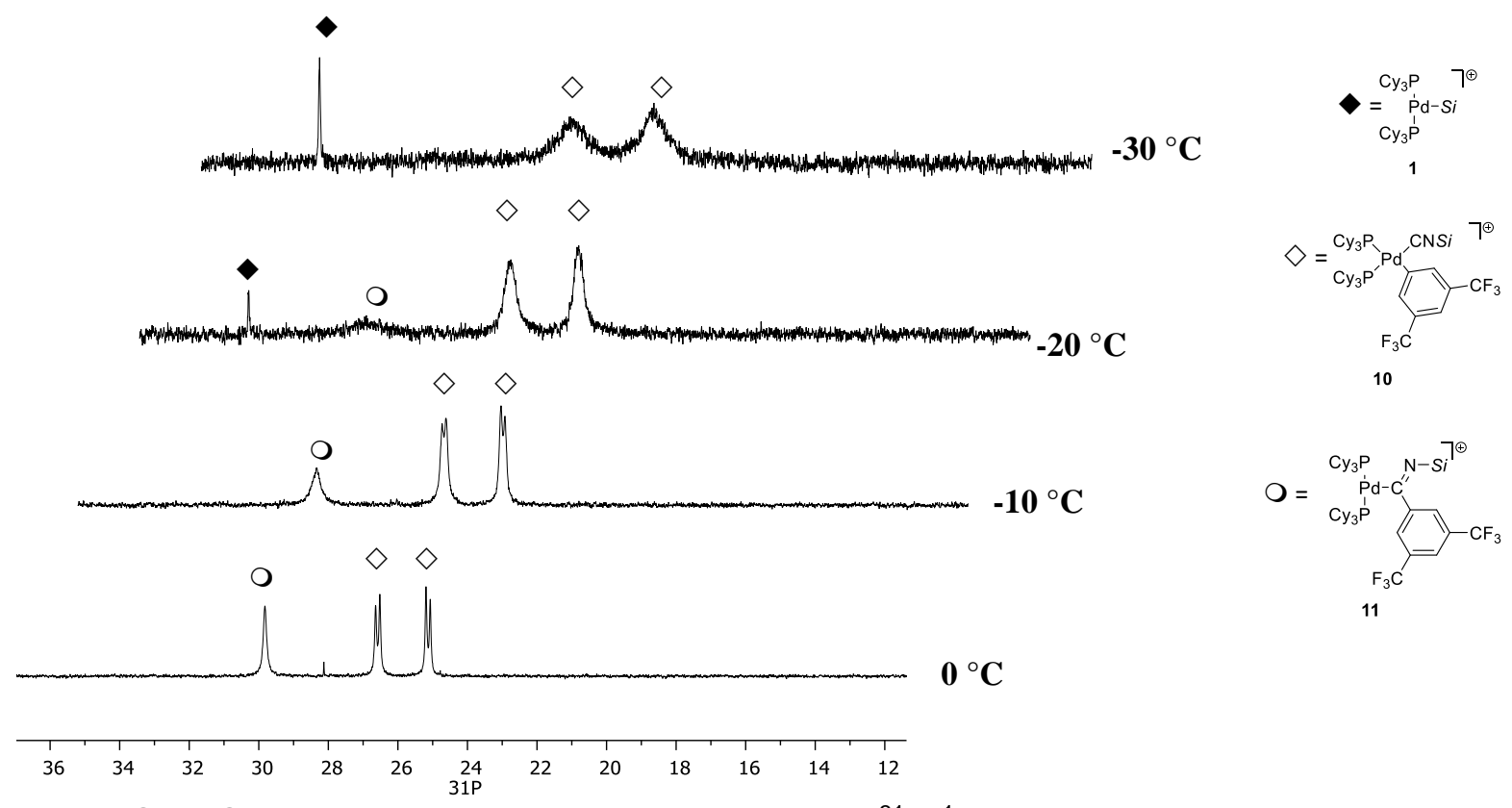

Figure S19: Selected $202 \mathrm{MHz}$ variable-temperature ${ }^{31} \mathrm{P}\left\{{ }^{1} \mathrm{H}\right\}$ NMR spectra for the reaction which generated 10 and 11 by method $b$, emphasizing $10 \& 11$ as the major products at $-30^{\circ} \mathrm{C}$. Solvent: 1:5 DFB:Tol- $d_{8} . \diamond=\mathbf{1}, \diamond=\mathbf{1 0}, O=11$.

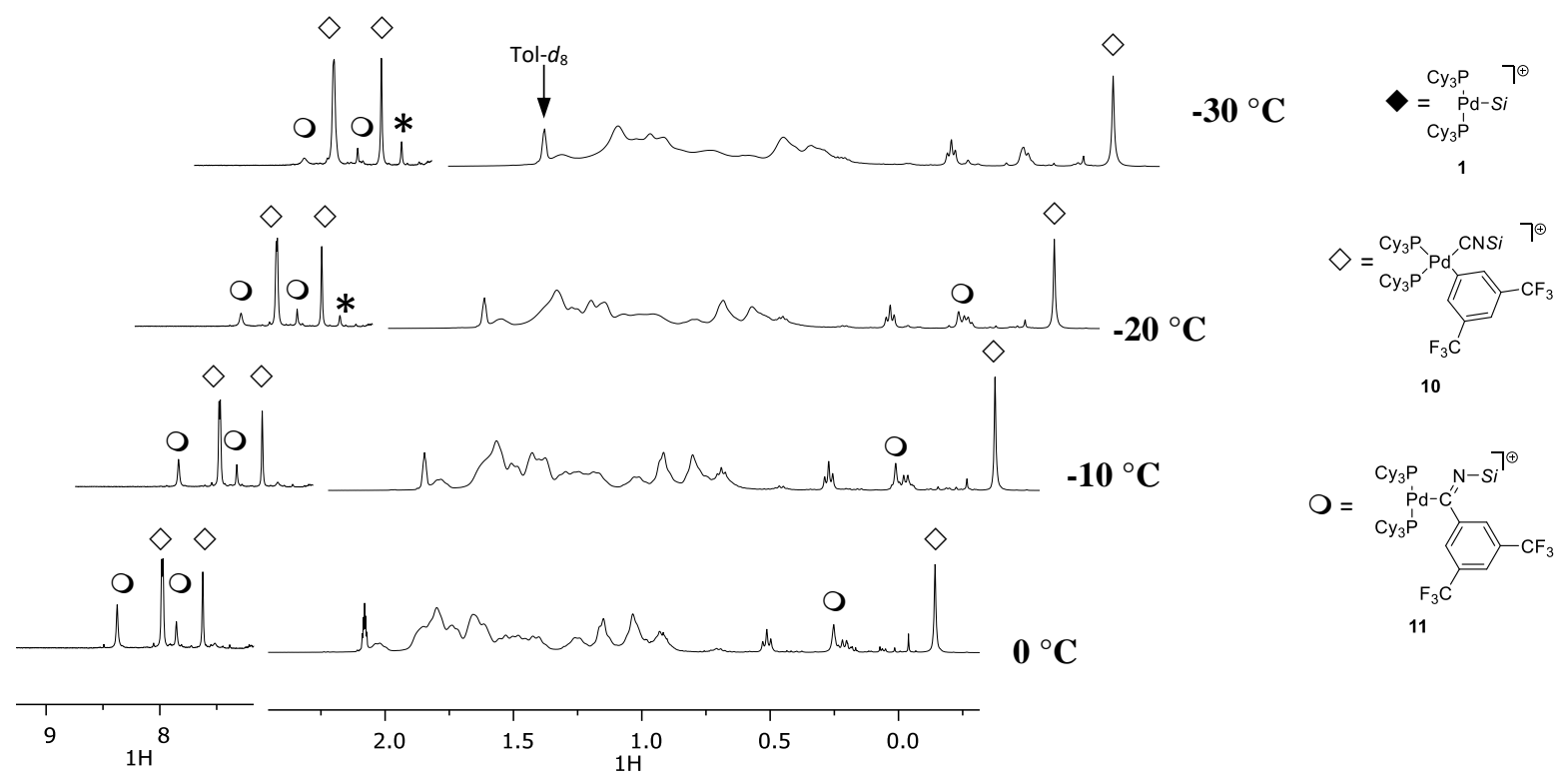

Figure S20: Selected $400 \mathrm{MHz}$ variable temperature ${ }^{1} \mathrm{H}$ NMR spectra for the reaction which generated 10 and 11 by method $b$, emphasizing $10 \& 11$ as the major products at $-30{ }^{\circ} \mathrm{C}$. The aromatic and $-\mathrm{SiMe}_{2} \mathrm{Et}$ protons are noted. Solvent: 1:5 DFB:Tol- $d_{8} . \diamond=10, O=11,{ }^{*}=\mathrm{NCArF}_{6}$. 


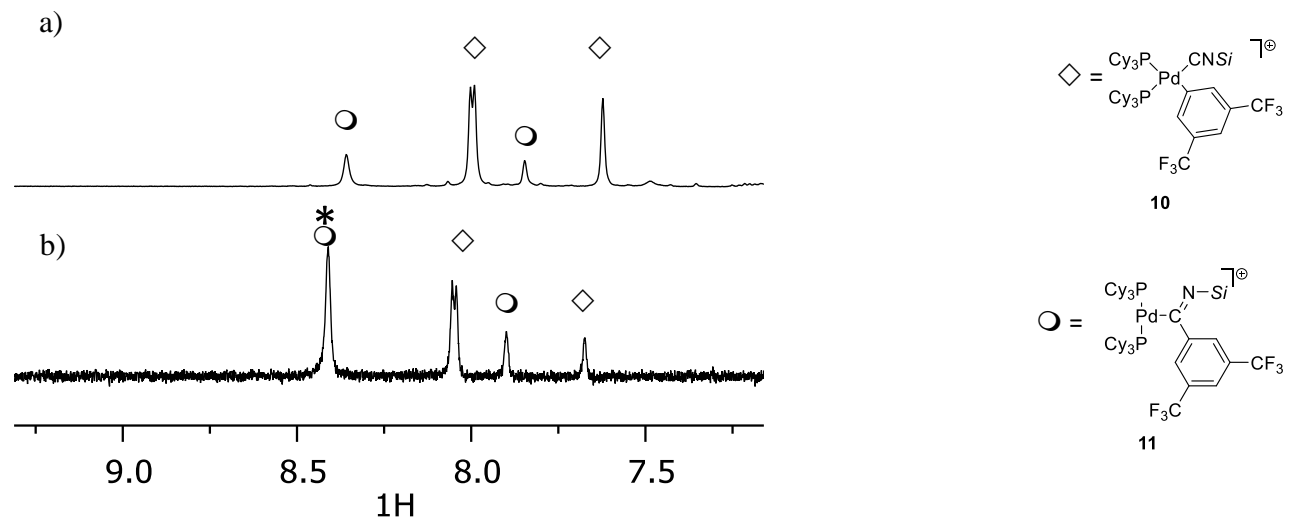

Figure S21: 1-D TOCSY experiments applied to a solution containing $\mathbf{1 0}$ and 11 at $-10{ }^{\circ} \mathrm{C}$, highlighting the presentation of aromatic resonances for both 10 and 11 ( $t_{\text {mix }}=80 \mathrm{~ms}$ ). a) Normal $500 \mathrm{MHz}{ }^{1} \mathrm{H}$ NMR spectrum. b) With selective excitation at 8.41 ppm*. Solvent: 1:5 DFB:Tol- $d_{8}$. Temperature: $-10^{\circ} \mathrm{C} . \diamond=10, \bigcirc=11$

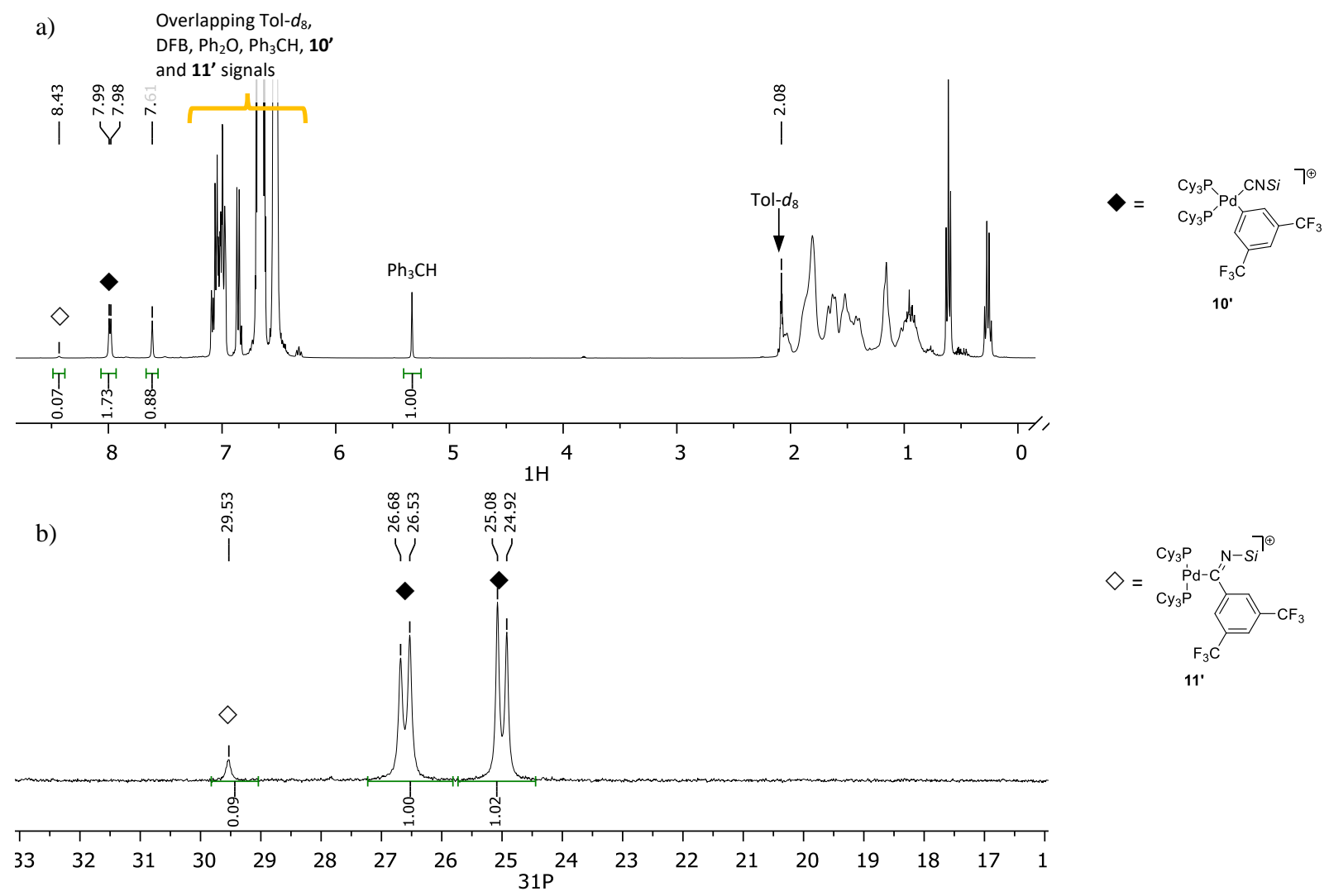

Figure S22: Selected NMR spectra at $-10^{\circ} \mathrm{C}$ for the in situ generation of 10' and 11' following method $b$, highlighting the ratio of 10' to 11'. a) $400 \mathrm{MHz}{ }^{1} \mathrm{H}$ NMR spectrum. b) $162 \mathrm{MHz}{ }^{31} \mathrm{P}\left\{{ }^{1} \mathrm{H}\right\}$ NMR spectrum. Solvent: 1:5 DFB:Tol- $d_{8} . \diamond=10^{\prime}, \diamond=11$ '. 


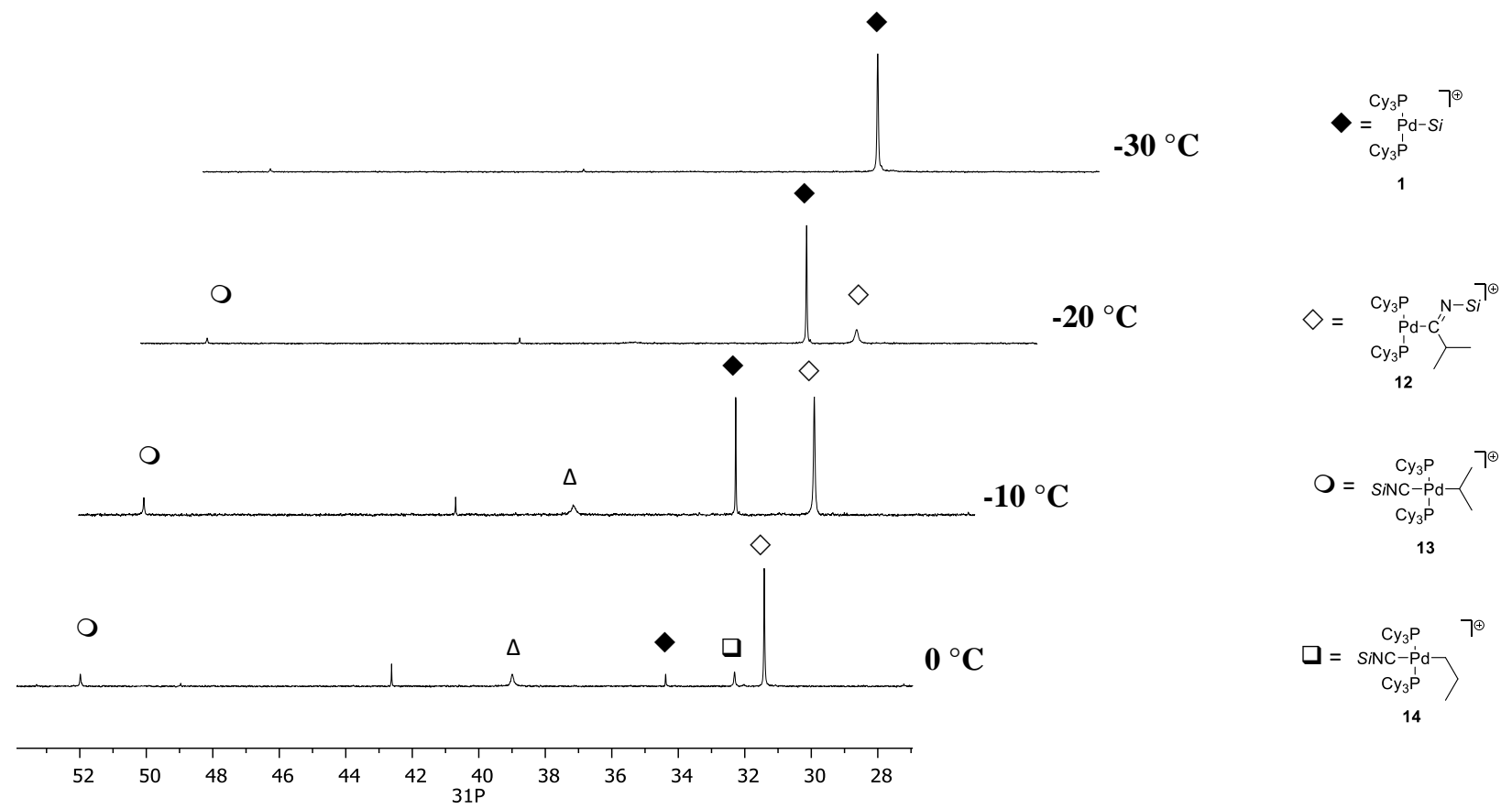

Figure S23: Selected $202 \mathrm{MHz}$ variable-temperature ${ }^{31} \mathrm{P}\left\{{ }^{1} \mathrm{H}\right\}$ NMR spectra for the reaction which generated 12, 13, 14, and $\mathrm{Pd}\left(\mathrm{PC}_{3}\right)_{2}$ from 1 by method $a$. This reaction highlights the conversion of 1 to the aforementioned species upon warming from $-30^{\circ} \mathrm{C}$. Solvent: 1:5 DFB:Tol- $d_{8} . \downarrow=1$, $\diamond=12, \bigcirc=13, \square=14, \Delta=\operatorname{Pd}\left(\mathrm{PCy}_{3}\right)_{2}$.

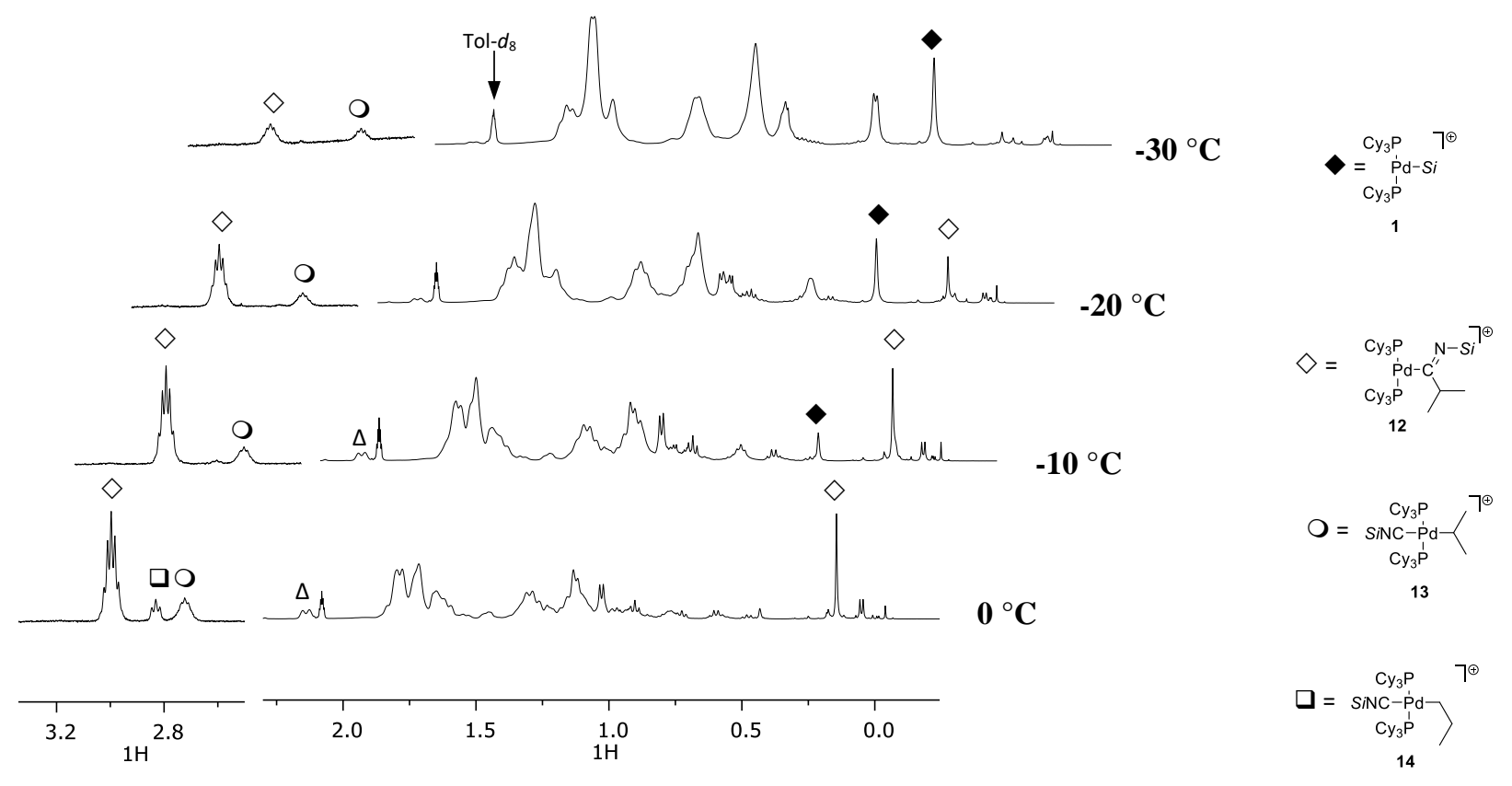

Figure S24: Selected $400 \mathrm{MHz}$ variable temperature ${ }^{1} \mathrm{H}$ NMR spectra for the reaction which generated 12, 13, 14, and $\mathrm{Pd}\left(\mathrm{PCy}_{3}\right)_{2}$ by method $a$. This reaction highlights the conversion of 1 to the aforementioned species upon warming from $-30{ }^{\circ} \mathrm{C}$. The- $\mathrm{CHMe},-\mathrm{CH}_{2} \mathrm{CH}_{2} \mathrm{CH}_{3}$, and $\mathrm{SiMe}_{2}$ Et protons are noted. Solvent: 1:5 DFB:Tol- $d_{8} . \diamond=1, \diamond=12, O=13, \square=14, \Delta=\operatorname{Pd}(\operatorname{PCy})_{2}$. 


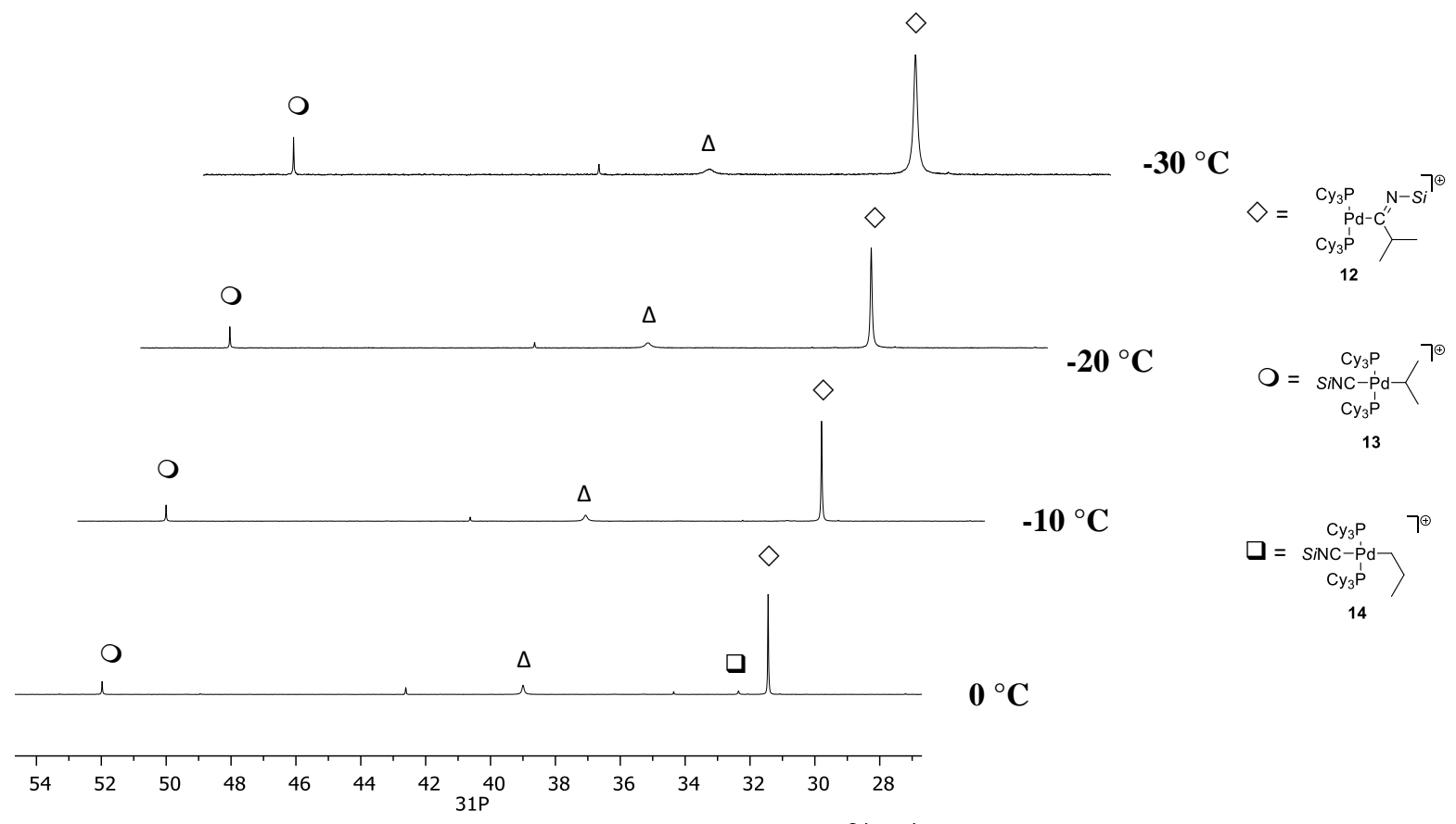

Figure S25: Selected $202 \mathrm{MHz}$ variable-temperature ${ }^{31} \mathrm{P}\left\{{ }^{1} \mathrm{H}\right\}$ NMR spectra for the reaction which generated 12, 13, 14, $\mathrm{Pd}\left(\mathrm{PCy}_{3}\right)_{2}$, by method $b$, highlighting the complete consumption of 1 at -30 ${ }^{\circ} \mathrm{C}$. Solvent: 1:5 DFB:Tol- $d_{8} . \diamond=12, \mathrm{O}=13, \square=14, \Delta=\mathrm{Pd}\left(\mathrm{PCy}_{3}\right)_{2}$.

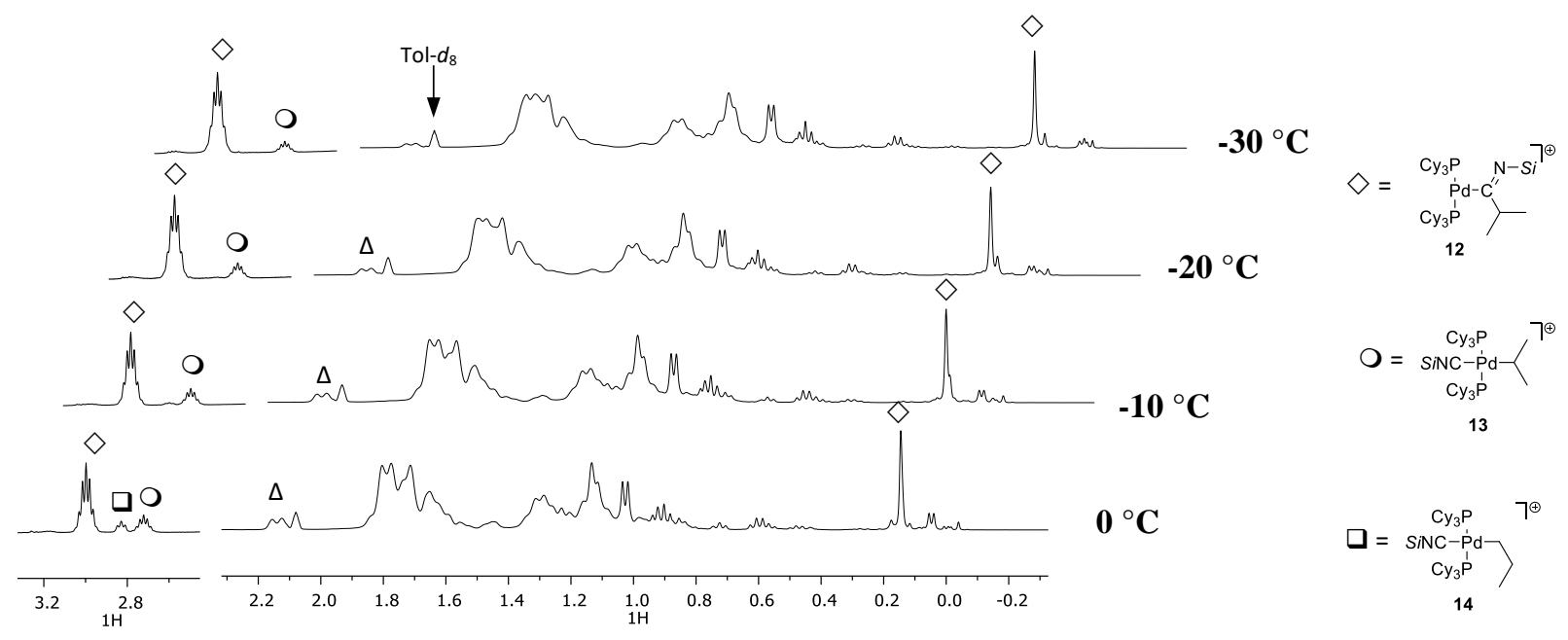

Figure S26: Selected $400 \mathrm{MHz}$ variable temperature ${ }^{1} \mathrm{H}$ NMR spectra for the reaction which generated 12, 13, 14, and $\mathrm{Pd}\left(\mathrm{PCy}_{3}\right)_{2}$, by method $b$, highlighting the complete consumption of 1 at $-30^{\circ} \mathrm{C}$. The isopropyl and $-\mathrm{SiMe} 2 \mathrm{Et}$ protons are noted. Solvent: 1:5 DFB:Tol- $d_{8} . \diamond=\mathbf{1 2}, \mathrm{O}=\mathbf{1 3}$, $\square=14, \Delta=\operatorname{Pd}\left(\mathrm{PCy}_{3}\right)_{2}$. 


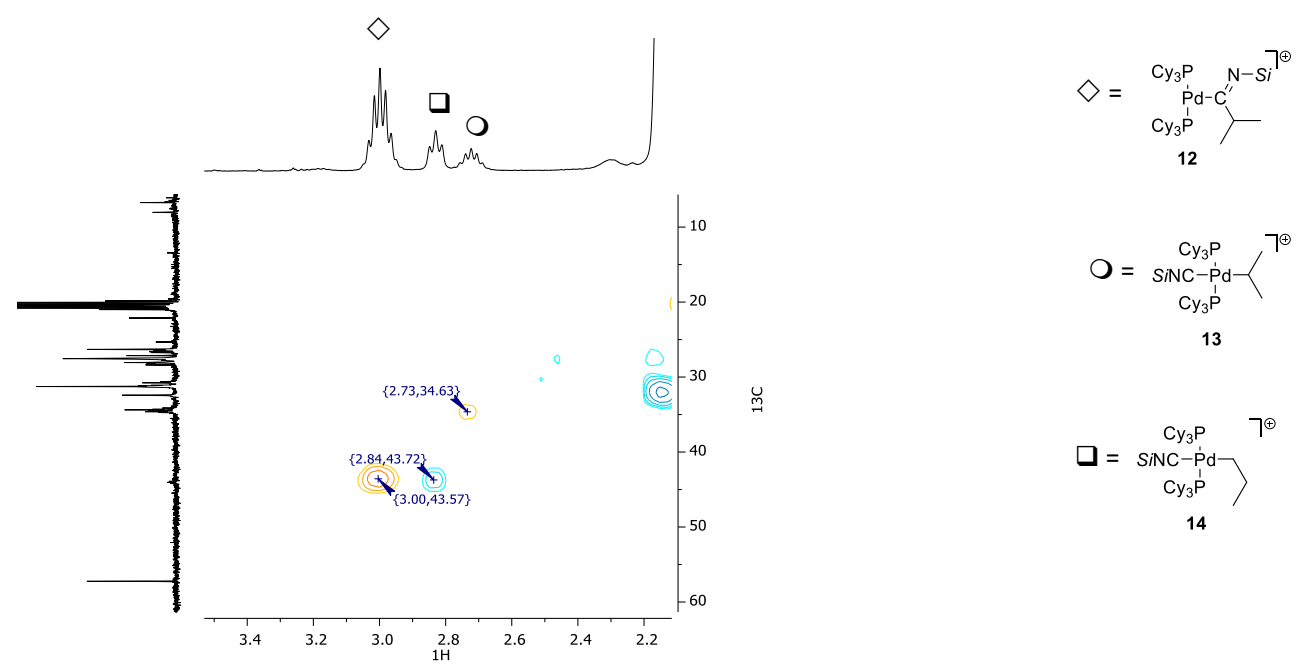

Figure S27: A phased H,C-HSQC $(400 \mathrm{MHz}, 100 \mathrm{MHz})$ of a solution containing 12, 13, and 14 at $0{ }^{\circ} \mathrm{C}$, highlighting the location and phase of selected carbon resonances. Solvent: 1:5 DFB:Tol$d_{8} . \diamond=12, \bigcirc=13, \square=14$.

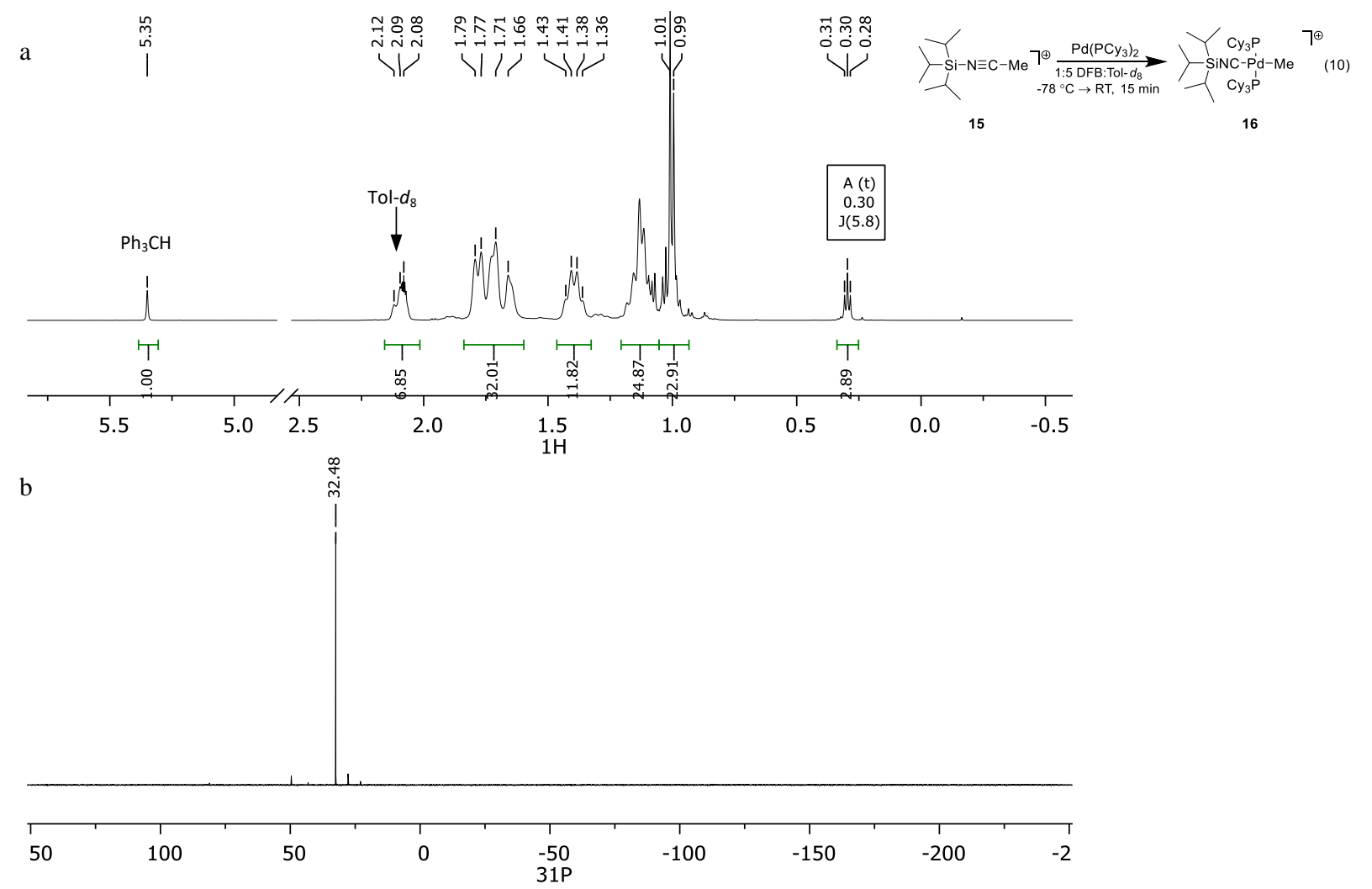

Figure S28: Selected room temperature NMR spectra acquired within minutes after the addition

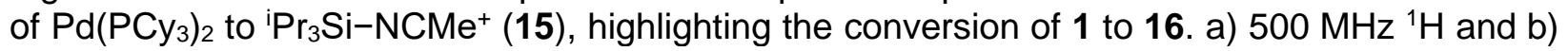
$202 \mathrm{MHz}{ }^{31} \mathrm{P}\left\{{ }^{1} \mathrm{H}\right\}$ NMR spectra. Solvent: 1:5 DFB:Tol- $d_{8}$. 


\section{HRMS Data}

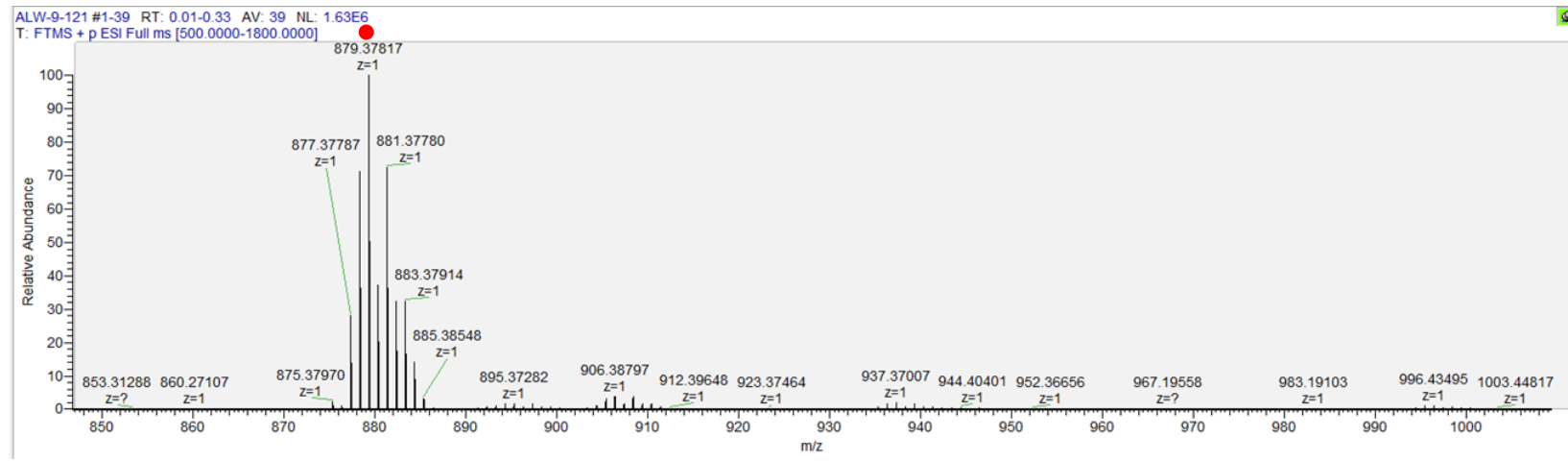

Figure S29: HESI HRMS for 2', highlighting the [2'-CN] ${ }^{+}$ion.

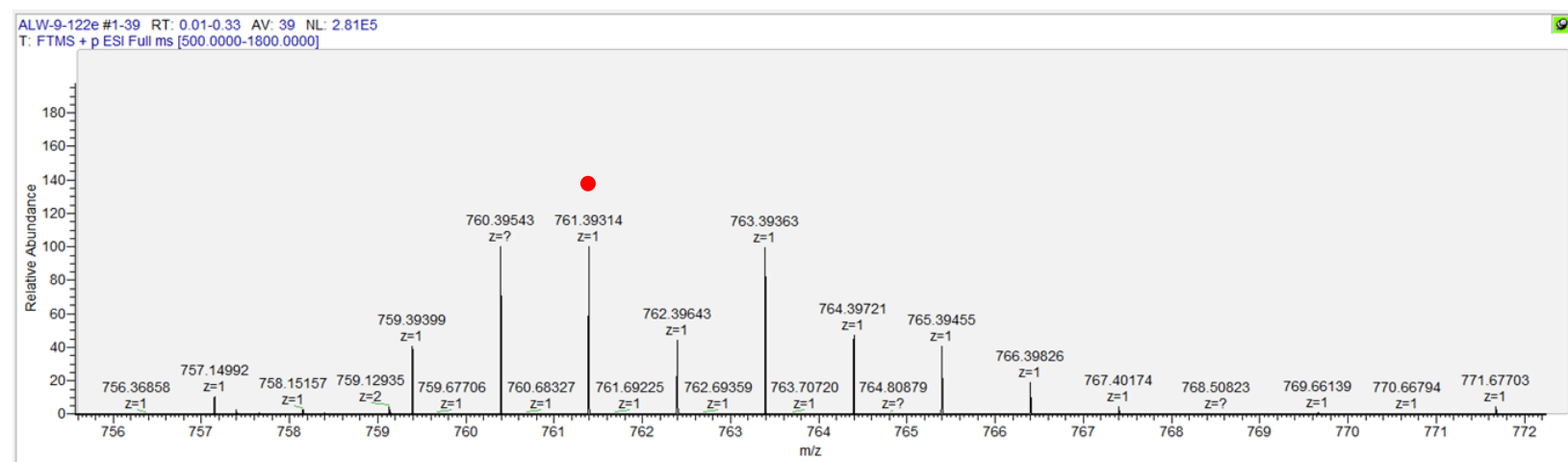

Figure S30: HESI HRMS for 3', highlighting the [3'-CN] ${ }^{+}$ion.

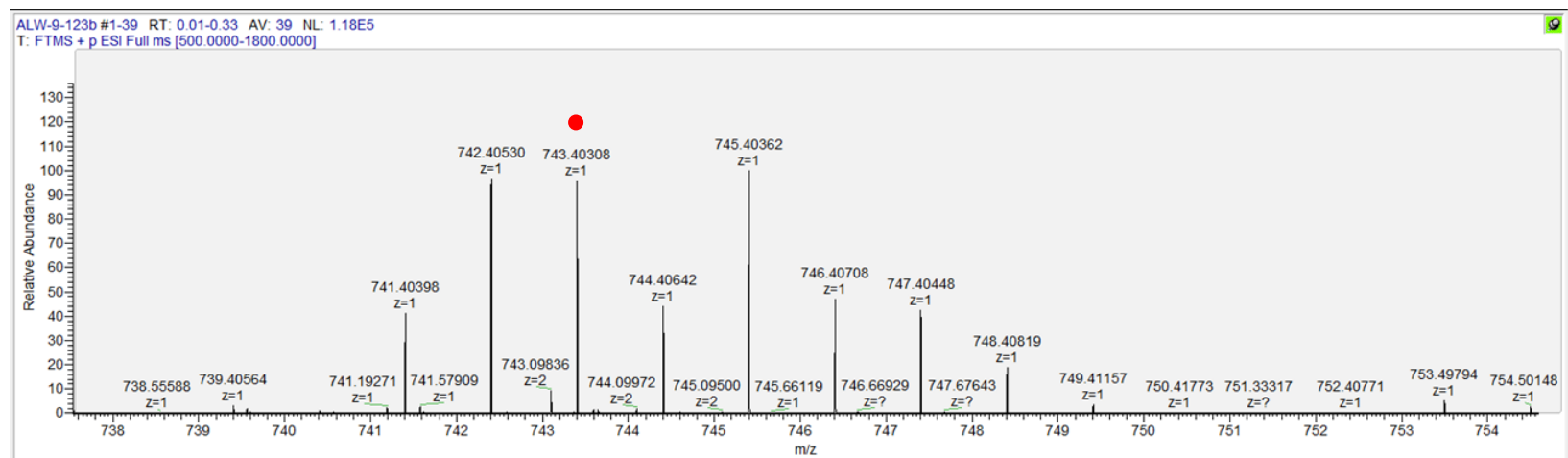

Figure S31: HESI HRMS for 4', highlighting the [4'-CN]+ ion. 


\section{Computational Method}

\section{Computational Data}

All DFT calculations were carried out in the gas phase with the B3LYP functional using the Gaussian 09 program in the absence of the $\mathrm{B}\left(\mathrm{C}_{6} \mathrm{~F}_{5}\right)_{4}{ }^{-}$counteranion. ${ }^{5}$ Ground state geometry optimizations were performed with the LANL2DZ basis set for the Pd atom and $6-31 \mathrm{G}(\mathrm{d}, \mathrm{p})$ for the other atoms. Vibrational frequencies were calculated analytically at the same level of theory to confirm the nature of structures and to calculate the Gibbs free $(\Delta G)$ energy corrections. Interaction energies were calculated using the same level of theory, with the two counterpoise fragments assigned as the singly charged silylium moiety and the corresponding neutral palladium complex. The relative intrinsic silylicity $(\Pi)$ is defined similarly to that reported by Djukic and is the ratio of the interfragment interaction energy of triflate with the silylium group, to the interaction energy of the neutral metal complex with the same silylium moiety. ${ }^{6}$

The supplemental file [DFT GS Coord] contains the computed Cartesian coordinates of all of the molecules reported in this study. The file may be opened as a text file to read the coordinates, or opened directly by a molecular modeling program such as Mercury (version 3.3 or later, http://www.ccdc.cam.ac.uk/pages/Home.aspx) for visualization and analysis.

Table S1: Calculated silylicity (ח) and metrical parameters for the ground state DFT optimized geometries for selected compounds.

\begin{tabular}{|c|c|c|c|c|c|c|c|c|}
\hline Compound & $\begin{array}{c}\text { Silylicity } \\
\text { (П) }\end{array}$ & $\begin{array}{l}\text { P-Pd-P } \\
\text { (deg) }\end{array}$ & $\begin{array}{c}\text { Pd-C-N } \\
\text { (deg) }\end{array}$ & $\begin{array}{l}\text { Pd-P1 } \\
(\AA ̊)\end{array}$ & $\begin{array}{c}\mathrm{Pd}-\mathrm{P2} \\
(\AA \AA)\end{array}$ & $\begin{array}{l}\mathrm{Pd}-\mathrm{C} \\
(\AA \AA)\end{array}$ & $\begin{array}{l}\mathrm{Pd}-\mathrm{N} \\
(\AA ̊)\end{array}$ & $\phi$ (deg) \\
\hline 1 & 1.76 & 156.03 & NA & 2.4637 & 2.4172 & NA & NA & NA \\
\hline 6 & 1.6 & 112.15 & 85.56 & 2.392 & 2.608 & 2.004 & 2.276 & 11.16 \\
\hline 7 & 1.49 & 159.45 & 118.55 & 2.4507 & 2.4514 & 2.0461 & 2.8432 & 85.9 \\
\hline 8 & 1.47 & 159.16 & 118.05 & 2.4512 & 2.4502 & 2.0503 & 2.8403 & 85.81 \\
\hline 11 & 1.57 & 159.61 & 120.05 & 2.4561 & 2.4417 & 2.0262 & 2.8469 & 76.33 \\
\hline 12 & 1.49 & 152.83 & 120.39 & 2.4691 & 2.4559 & 2.0472 & 2.8633 & 77.31 \\
\hline
\end{tabular}

Table S2: Calculated single point energy, virtual frequencies, and interaction energies for the ground state DFT optimized geometries for selected compounds.

\begin{tabular}{|c|c|c|c|}
\hline Compound & $\Delta G$ (a.u.) & $\begin{array}{c}\# \\
\text { imag } \\
\text { freq }\end{array}$ & $\begin{array}{c}\text { Interaction } \\
\text { energy } \\
\text { (corrected) } \\
\text { (kcal/mol) }\end{array}$ \\
\hline 1 & $2668 . \overline{6} 60386$ & 0 & -97.69 \\
\hline 6 & 2801.33816 & 0 & -107.15 \\
\hline 7 & 3092.27988 & 0 & -115.53 \\
\hline 8 & 2993.04202 & 0 & -117.18 \\
\hline 11 & 3667.10043 & 0 & -109.35 \\
\hline 12 & 2879.92 & 0 & -115.7 \\
\hline $\begin{array}{c}\text { TfO- } \\
\text { SiMe }_{2} \mathrm{Et}\end{array}$ & $\begin{array}{c}- \\
448.657913\end{array}$ & 0 & -171.84 \\
\hline
\end{tabular}




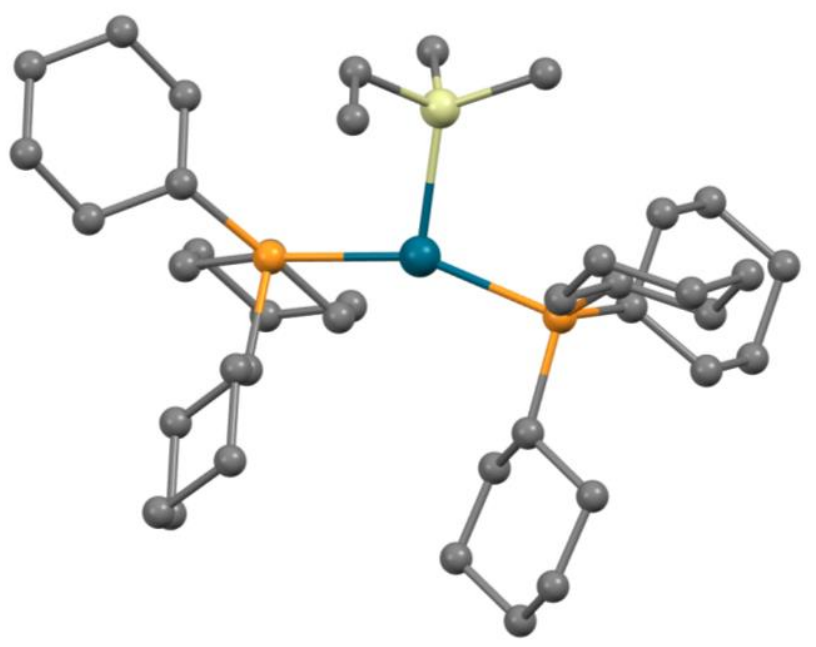

Figure S32: The ground state optimized geometry in the gas phase for 1 at the B3LYP level using split triple-zeta basis sets.

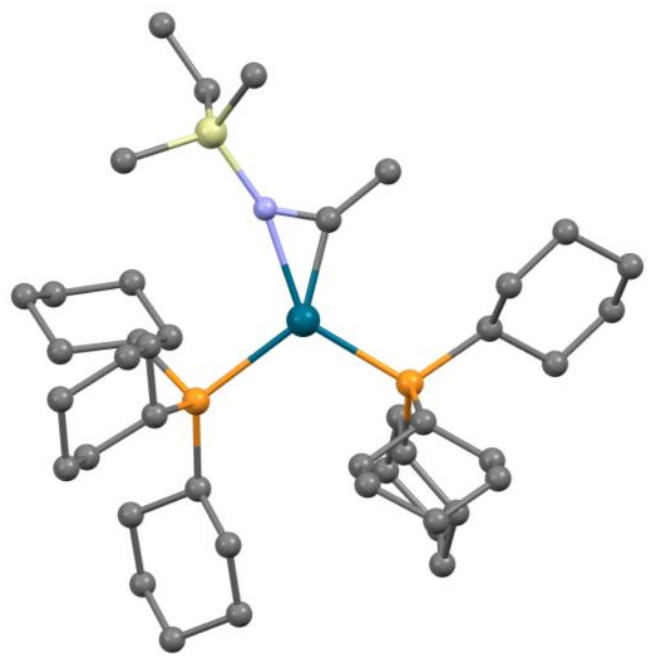

Figure S33: The ground state optimized geometry in the gas phase for 6 at the B3LYP level using split triple-zeta basis sets. 


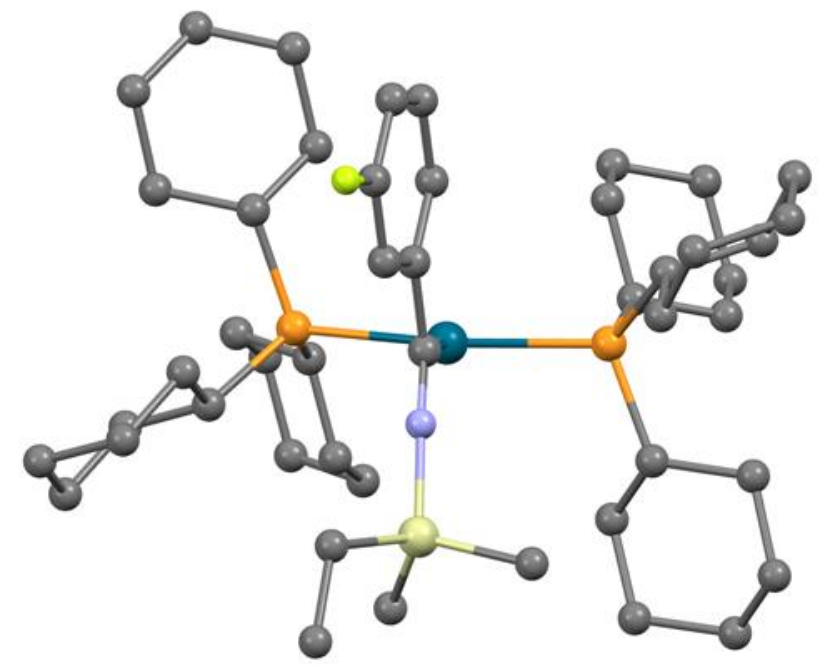

Figure S34: The ground state optimized geometry in the gas phase for 7 at the B3LYP level using split triple-zeta basis sets.

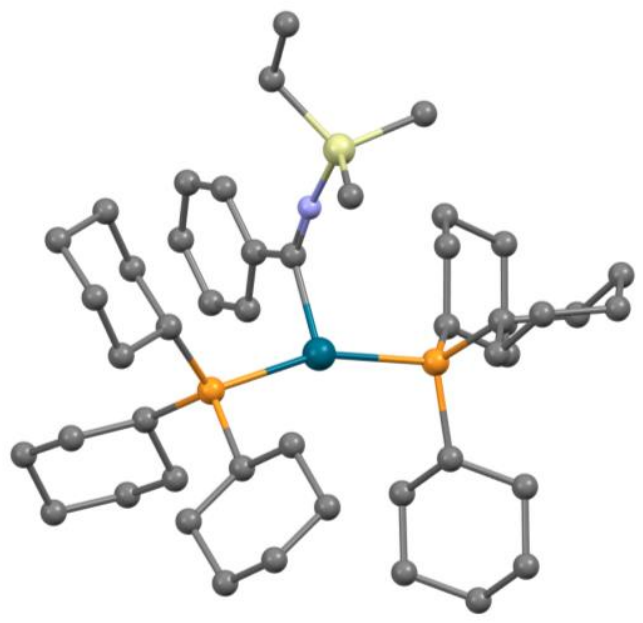

Figure S35: The ground state optimized geometry in the gas phase for $\mathbf{8}$ at the B3LYP level using split triple-zeta basis sets. 


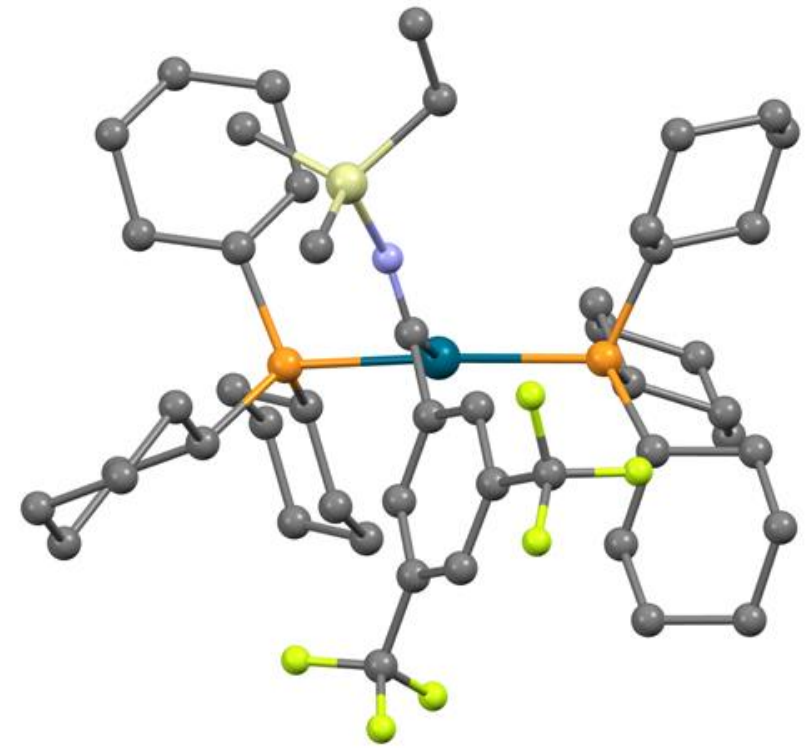

Figure S36: The ground state optimized geometry in the gas phase for $\mathbf{1 1}$ at the B3LYP level using split triple-zeta basis sets.

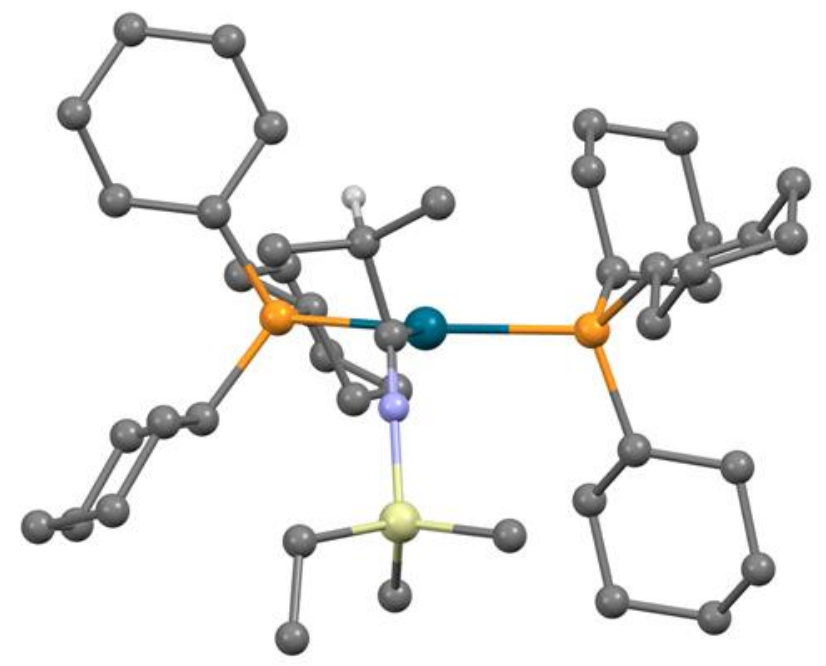

Figure S37: The ground state optimized geometry in the gas phase for $\mathbf{1 2}$ at the B3LYP level using split triple-zeta basis sets. 


\section{X-ray Diffraction Experimental Data}

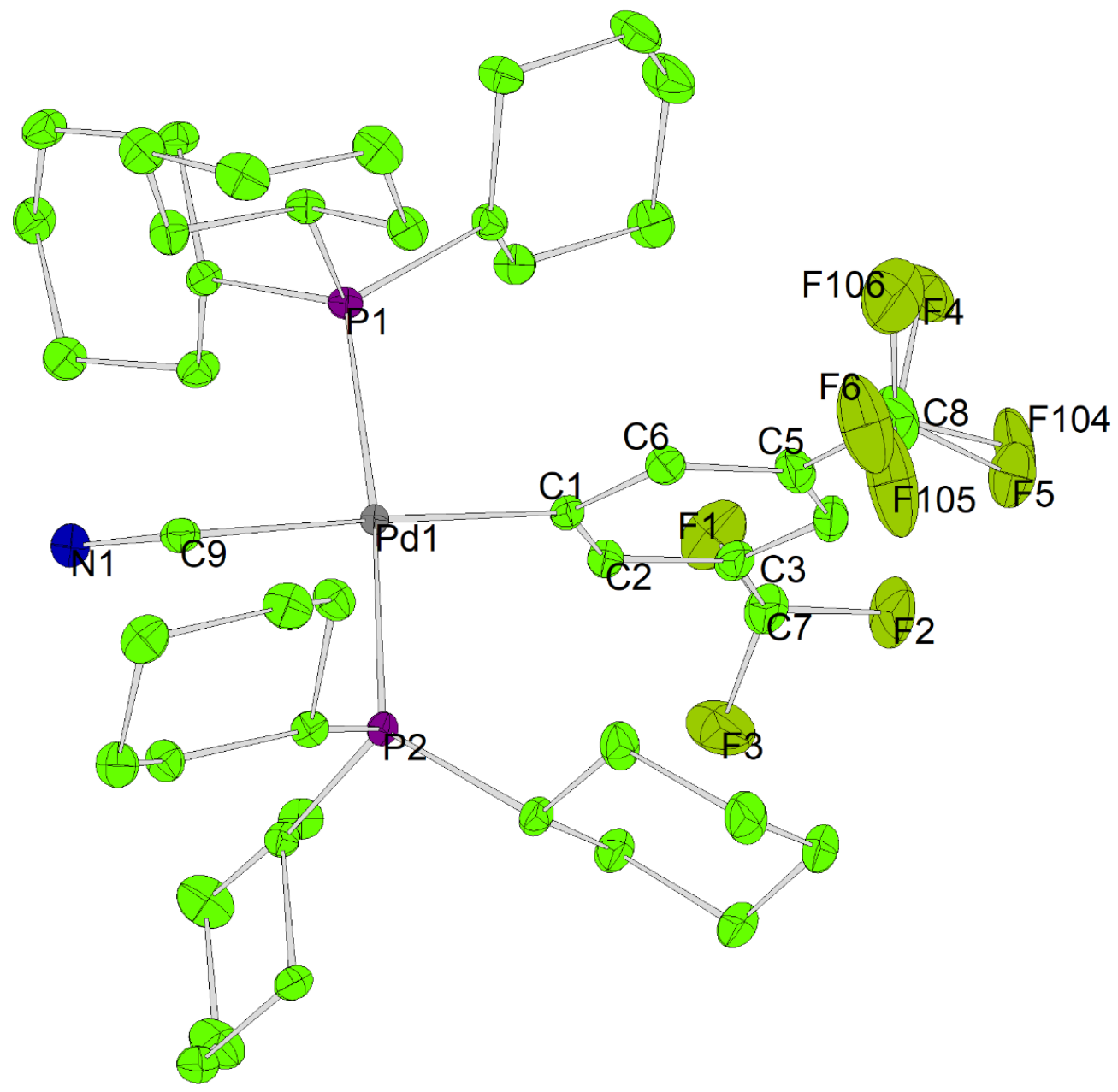

Figure S38: Graphics with numbering for key atoms for 2'. The carbons on the cyclohexyl phosphine ligands are numbered $\mathrm{C} 10-\mathrm{C} 27$ for $\mathrm{P} 1$, and $\mathrm{C} 28-45$ for $\mathrm{P} 2$. The graphic omits the second palladium complex also found in the unit cell, as well as hydrogen atoms. 
Table S3: Structure Solution and Refinement Details for 2'.

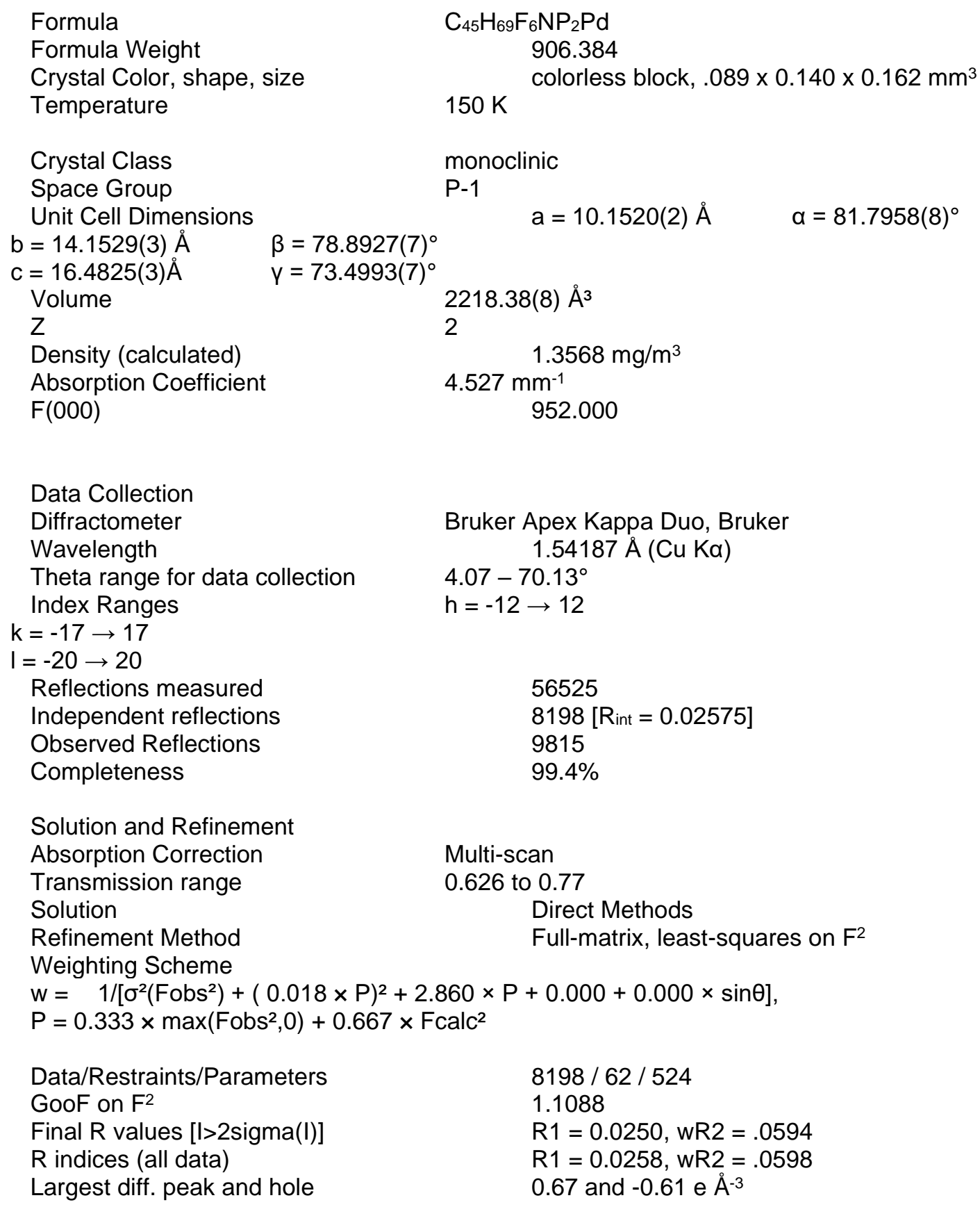

Formula

Formula Weight

Crystal Color, shape, size

Temperature

Crystal Class

Space Group

Unit Cell Dimensions

$\mathrm{b}=14.1529$ (3) $\AA$

$c=16.4825(3) \AA$

Volume

Z

Density (calculated)

Absorption Coefficient

$\mathrm{F}(000)$

Data Collection

Diffractometer

Wavelength

Theta range for data collection

Index Ranges

$\mathrm{k}=-17 \rightarrow 17$

$\mathrm{I}=-20 \rightarrow 20$

Reflections measured

Independent reflections

Observed Reflections

Completeness

Solution and Refinement

Absorption Correction

Transmission range

Solution

Refinement Method

Weighting Scheme

$\beta=78.8927(7)^{\circ}$

$Y=73.4993(7)^{\circ}$

$\mathrm{C}_{45} \mathrm{H}_{69} \mathrm{~F}_{6} \mathrm{NP}_{2} \mathrm{Pd}$

906.384

colorless block, $.089 \times 0.140 \times 0.162 \mathrm{~mm}^{3}$

$150 \mathrm{~K}$

monoclinic

P-1

$$
a=10.1520(2) \AA \quad \alpha=81.7958(8)^{\circ}
$$

2218.38(8) $\AA^{3}$

2

$4.527 \mathrm{~mm}^{-1}$

$1.3568 \mathrm{mg} / \mathrm{m}^{3}$

952.000

Bruker Apex Kappa Duo, Bruker

$4.07-70.13^{\circ}$ $1.54187 \AA$ ( Cu Ka)

$\mathrm{h}=-12 \rightarrow 12$

56525

$8198\left[R_{\text {int }}=0.02575\right]$

9815

$99.4 \%$

Multi-scan

0.626 to 0.77

Direct Methods

Full-matrix, least-squares on $\mathrm{F}^{2}$

$\mathrm{W}=1 /\left[\sigma^{2}\left(\right.\right.$ Fobs $\left.\left.^{2}\right)+(0.018 \times \mathrm{P})^{2}+2.860 \times \mathrm{P}+0.000+0.000 \times \sin \theta\right]$,

$\mathrm{P}=0.333 \times \max \left(\right.$ Fobs $\left.^{2}, 0\right)+0.667 \times$ Fcalc $^{2}$

Data/Restraints/Parameters

8198 / 62 / 524

GooF on $\mathrm{F}^{2}$

1.1088

Final $R$ values [l>2sigma(I)]

$\mathrm{R} 1=0.0250, \mathrm{wR} 2=.0594$

$\mathrm{R}$ indices (all data)

Largest diff. peak and hole

$\mathrm{R} 1=0.0258, \mathrm{wR} 2=.0598$

0.67 and -0.61 e $\AA^{-3}$ 


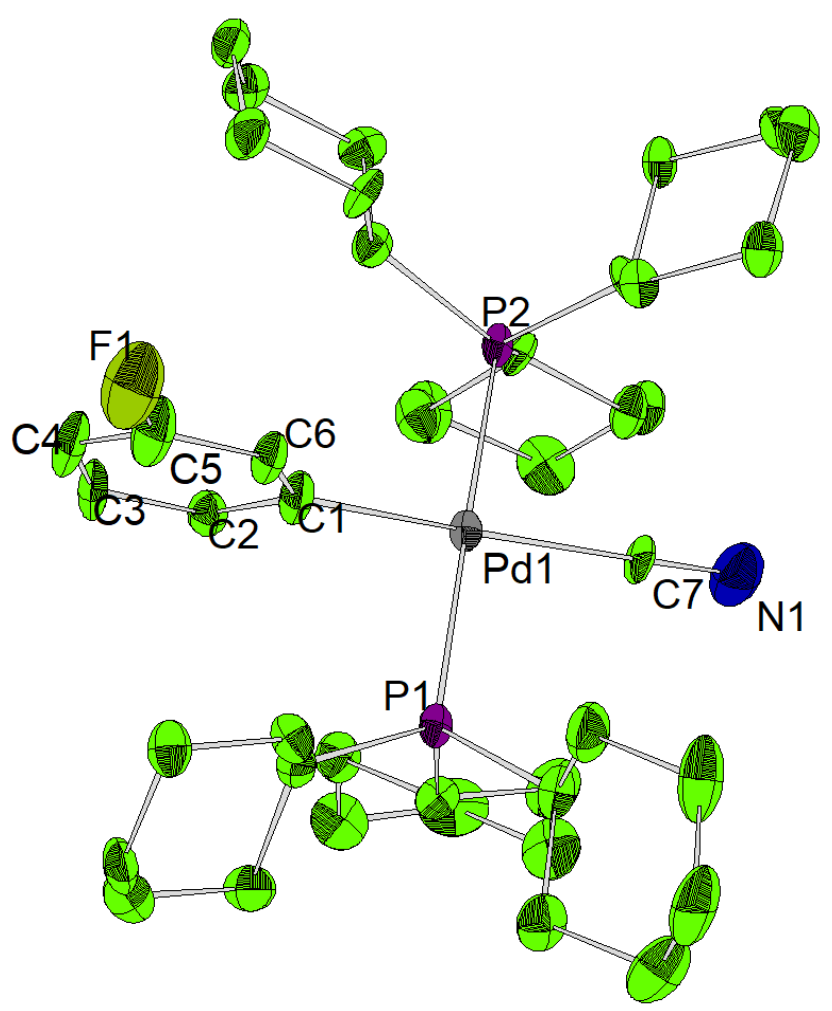

Figure S39: Graphics with numbering for key atoms for 3'. The carbons on the cyclohexyl phosphine ligands are numbered $\mathrm{C} 8-\mathrm{C} 25$ for $\mathrm{P} 1$, and $\mathrm{C} 26-43$ for $\mathrm{P} 2$. The graphic omits the second palladium complex and the solvent molecules also found in the unit cell, as well as hydrogen atoms. 
Table S4: Structure Solution and Refinement Details for 3'.

Formula

Formula Weight

Crystal Color, shape, size

Temperature

Crystal Class

Space Group

Unit Cell Dimensions

$\mathrm{b}=9.6481(3) \AA$

$\mathrm{C}=16.7362(5) \AA \quad \mathrm{A}=90^{\circ}$

Volume

Z

Density (calculated)

Absorption Coefficient

$\mathrm{F}(000)$
$\mathrm{C}_{44} \mathrm{H}_{72} \mathrm{Cl}_{2} \mathrm{FNP}_{2} \mathrm{Pd}$

873.312

colorless block fragment, . $114 \times 0.116 \times 0.260 \mathrm{~mm}^{3}$

$150 \mathrm{~K}$

monoclinic

P2 1

$$
a=13.8879(4) \AA \quad \alpha=90^{\circ}
$$

\section{Data Collection}

Diffractometer

Wavelength

Theta range for data collection

Index Ranges

$\mathrm{k}=-11 \rightarrow 11$

$\mathrm{I}=-18 \rightarrow 20$

Reflections measured

Independent reflections

Observed Reflections

Completeness to theta

Solution and Refinement

Absorption Correction

Transmission range

Solution

Refinement Method

Weighting Scheme

$\mathrm{W}=1 /\left[\sigma^{2}\left(\right.\right.$ Fobs $\left.\left.^{2}\right)+(0.080 \times \mathrm{P})^{2}+34.435 \times \mathrm{P}+0.000+0.000 \times \sin \theta\right]$,

$\mathrm{P}=0.333 \times \max \left(\right.$ Fobs $\left.^{2}, 0\right)+0.667 \times$ Fcalc $^{2}$

Data/Restraints/Parameters

GooF on $\mathrm{F}^{2}$

Final $R$ values [l>2sigma(I)]

$R$ indices (all data)

Largest diff. peak and hole
Bruker Apex Kappa Duo, Bruker

$1.54187 \AA$ (Cu Ka)

$3.1824-72.3234^{\circ}$

$\mathrm{h}=-15 \rightarrow 17$

16540

$7476\left[\mathrm{R}_{\mathrm{int}}=0.0346\right]$

9871

$98.9 \%$

Multi-scan

0.5369 to 0.54268

Charge Flipping

Full-matrix, least-squares on $\mathrm{F}^{2}$ 


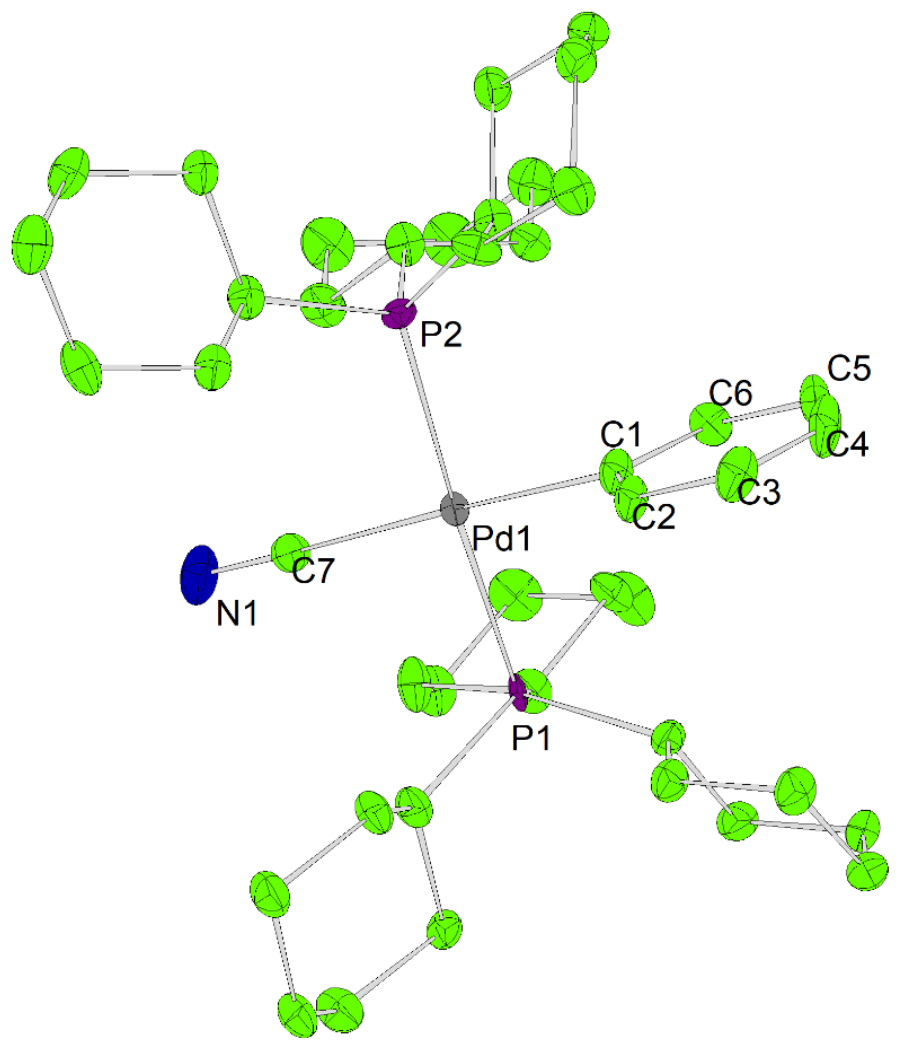

Figure S40: Graphics with numbering for key atoms for the 4'. The carbons on the cyclohexyl phosphine ligands are numbered $\mathrm{C} 8-\mathrm{C} 25$ for $\mathrm{P} 1$, and $\mathrm{C} 26-43$ for $\mathrm{P} 2$. The graphic omits the second palladium complex and the solvent molecules also found in the unit cell, as well as hydrogen atoms. 
Table S5: Structure Solution and Refinement Details for 4'.

Formula

Formula Weight

Crystal Color, shape, size

Temperature

Crystal Class

Space Group

Unit Cell Dimensions

$\mathrm{b}=9.6668(2) \AA$
$\mathrm{c}=16.5824(3) \AA \quad \mathrm{Y}$

Volume

Z

Density (calculated)

Absorption Coefficient

$\mathrm{F}(000)$

$\mathrm{C}_{44} \mathrm{H}_{73} \mathrm{Cl}_{2} \mathrm{NP}_{2} \mathrm{Pd}$

855.322

colorless block fragment, $.174 \times 0.238 \times 0.308 \mathrm{~mm}^{3}$

$150 \mathrm{~K}$

monoclinic

P2 1

$y=90^{\circ} \beta=90.0336(9)^{\circ}$

$$
a=13.8186(3) \AA \quad \alpha=90^{\circ}
$$

2

$1.282 \mathrm{mg} / \mathrm{m}^{3}$

$5.383 \mathrm{~mm}^{-1}$

908.000

\section{Data Collection}

Diffractometer

Wavelength

Theta range for data collection Index Ranges

$\mathrm{k}=-11 \rightarrow 11$

$\mathrm{I}=-20 \rightarrow 18$

Reflections measured

Independent reflections

Observed Reflections

Completeness

Bruker Apex Kappa Duo, Bruker

$1.54187 \AA$ (Cu Ka)

$4.1633-72.3981^{\circ}$

$\mathrm{h}=-17 \rightarrow 17$

18599

$7177\left[\mathrm{R}_{\text {int }}=0.0222\right]$

9914

$97.1 \%$

Solution and Refinement

Absorption Correction

Multi-scan

Transmission range

Solution

0.27773 to 0.39196

Refinement Method

Direct Methods

Weighting Scheme

Full-matrix, least-squares on $\mathrm{F}^{2}$

$w=1 /\left[\sigma^{2}\left(\right.\right.$ Fobs $\left.\left.^{2}\right)+(0.039 \times P)^{2}+24.920 \times P+0.000+0.000 \times \sin \theta\right]$,

$\mathrm{P}=0.333 \times \max \left(\right.$ Fobs $\left.^{2}, 0\right)+0.667 \times$ Fcalc $^{2}$

Data/Restraints/Parameters

GooF on $\mathrm{F}^{2}$

Final $R$ values [l>2sigma(I)]

$R$ indices (all data)

Largest diff. peak and hole
$7154 / 398 / 451$

1.0937

$\mathrm{R} 1=0.0683, \mathrm{wR} 2=.1702$

$R 1=0.0685, w R 2=.1703$

2.92 and $-2.07{\text { e } \AA^{-3}}^{-3}$ 
NMR Spectra for Reported Compounds.

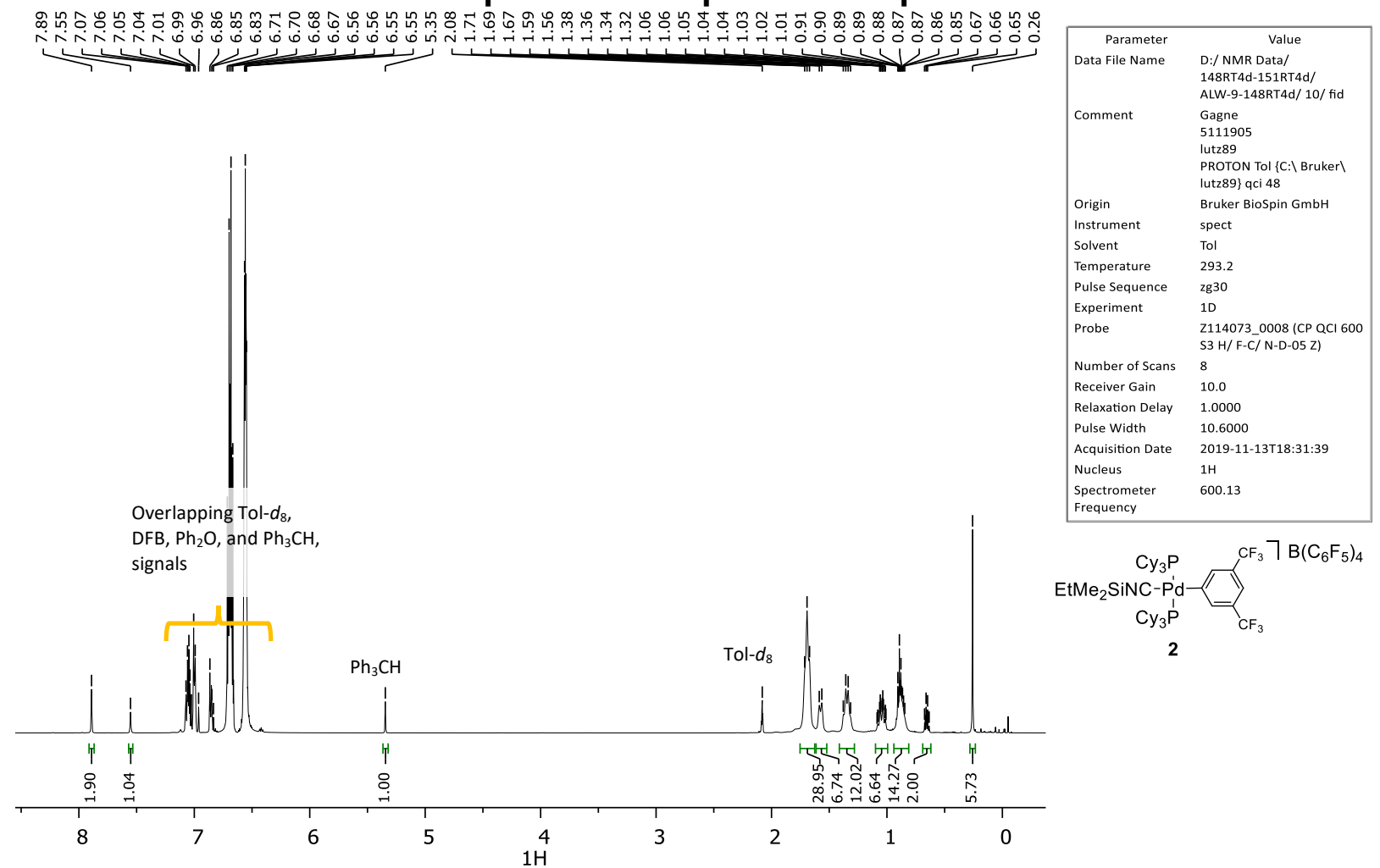

Figure S41: $600 \mathrm{MHz}{ }^{1} \mathrm{H}$ NMR spectrum of 2 at room temperature. Solvent: 1:5 DFB:Tol- $d_{8}$.

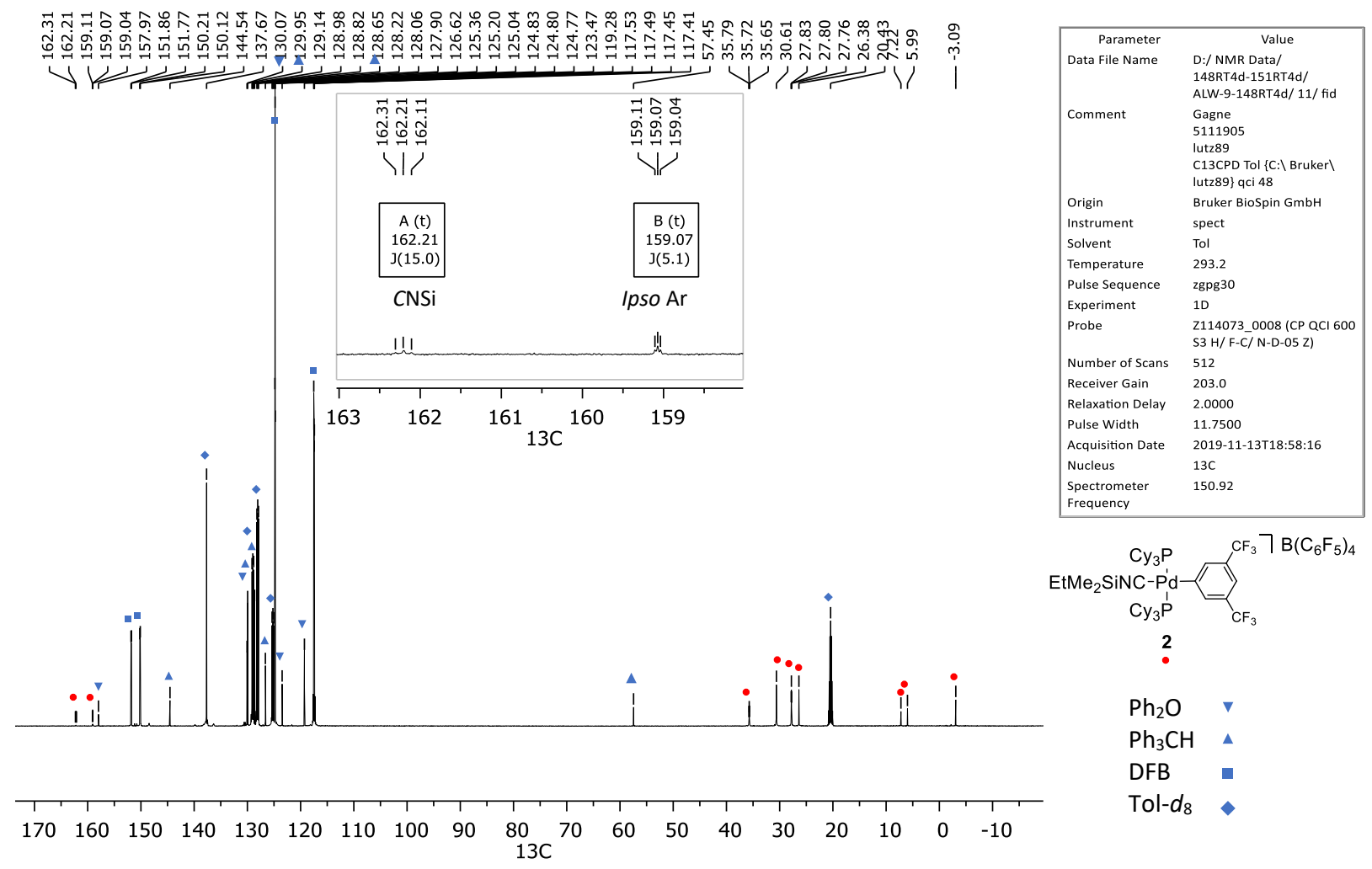

Figure S42: $151 \mathrm{MHz}{ }^{13} \mathrm{C}\left\{{ }^{1} \mathrm{H}\right\}$ NMR spectrum of 2 at room temperature. Solvent: 1:5 DFB:Tol- $d_{8}$. 


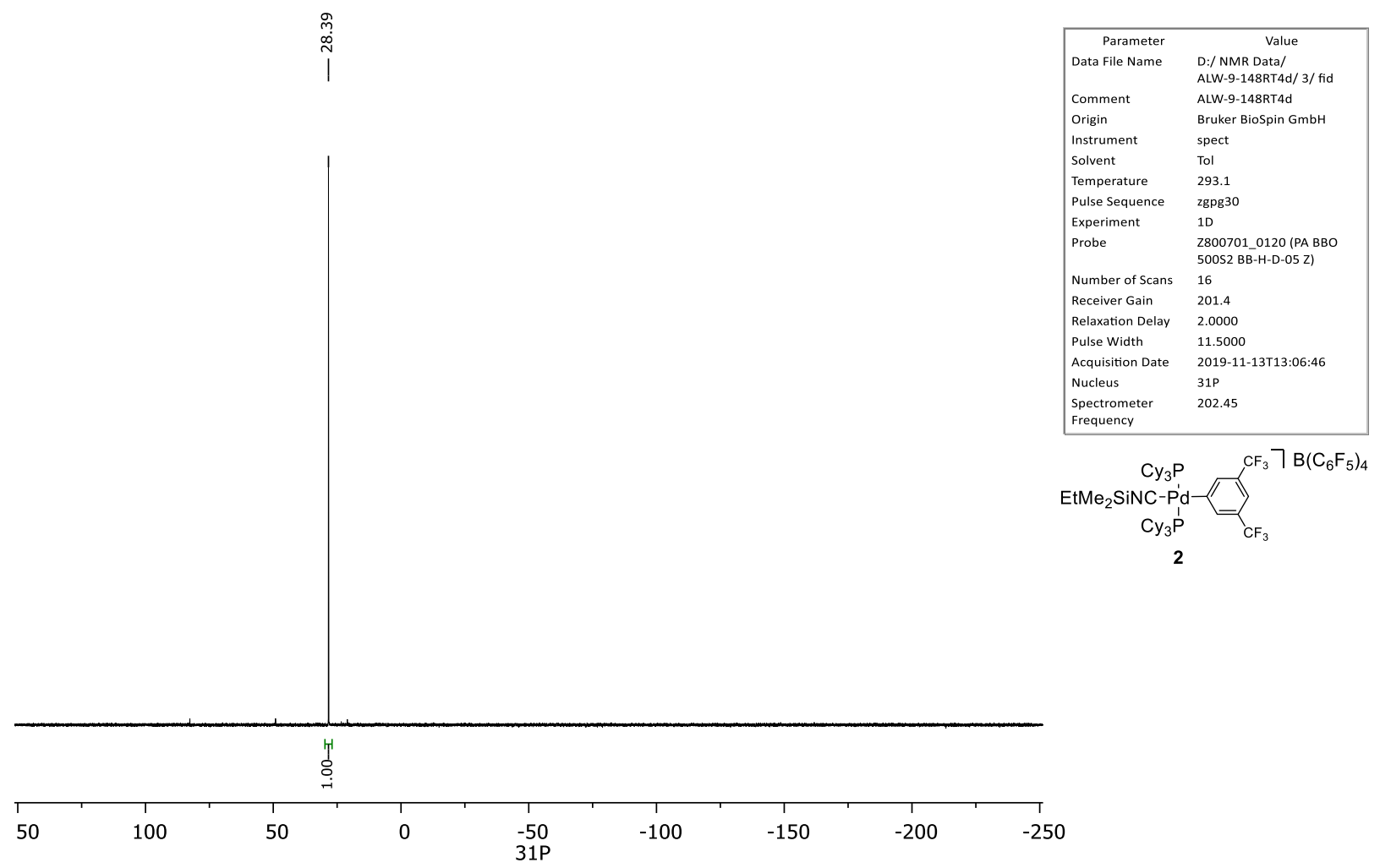

Figure S43: $202 \mathrm{MHz}{ }^{31} \mathrm{P}\left\{{ }^{1} \mathrm{H}\right\}$ NMR spectrum of 2 at room temperature. Solvent 1:5 DFB:Tol- $d_{8}$.

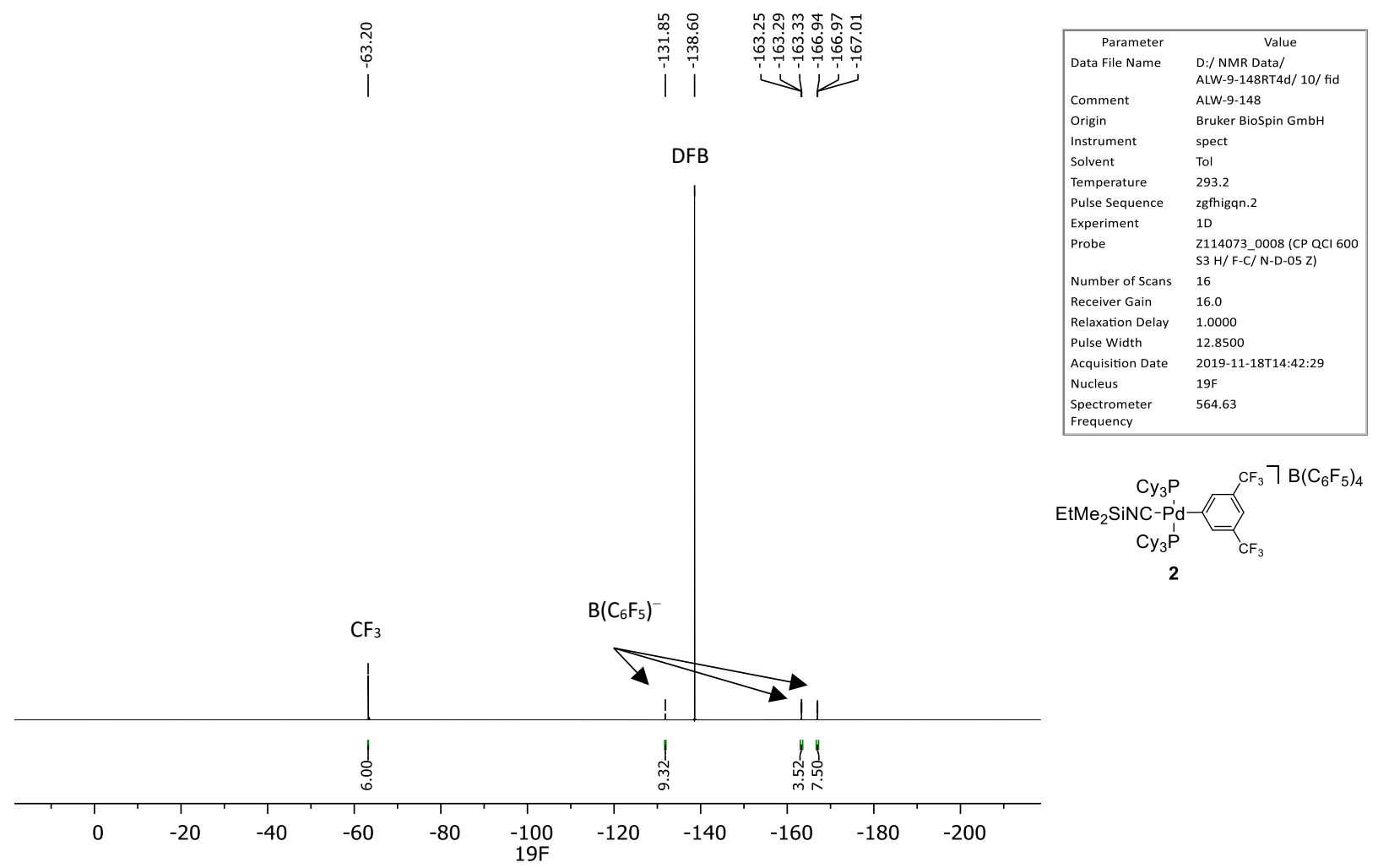

Figure S44: $565 \mathrm{MHz}{ }^{19} \mathrm{~F}\left\{{ }^{1} \mathrm{H}\right\}$ NMR spectrum of 2 at room temperature. Solvent: 1:5 DFB:Tol- $d_{8}$. 


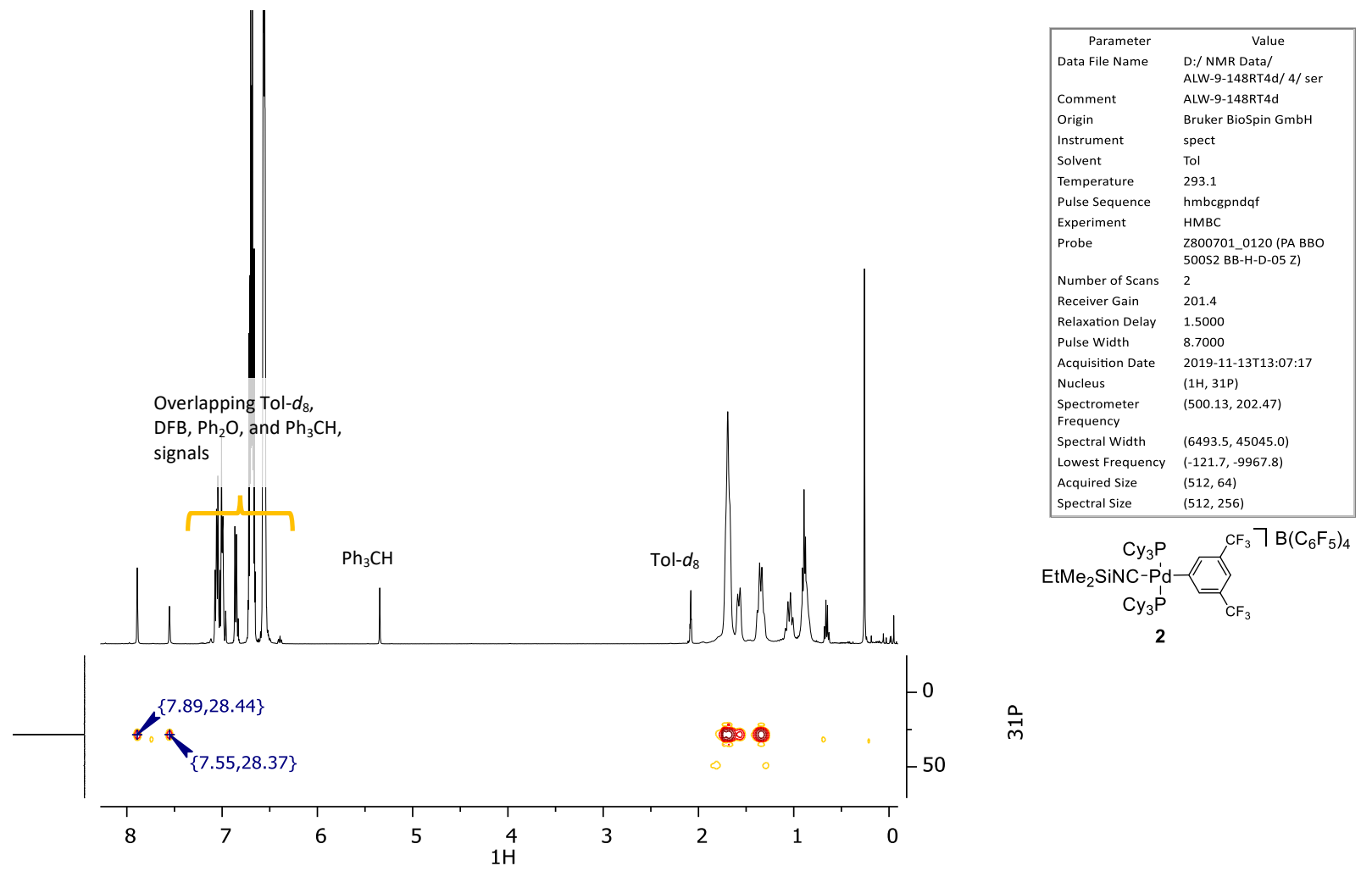

Figure S45: (500 MHz, $202 \mathrm{MHz}){ }^{1} \mathrm{H},{ }^{31} \mathrm{P}-\mathrm{HMBC}$ NMR spectrum of 2 at room temperature. Solvent: 1:5 DFB:Tol- $d_{8}$.

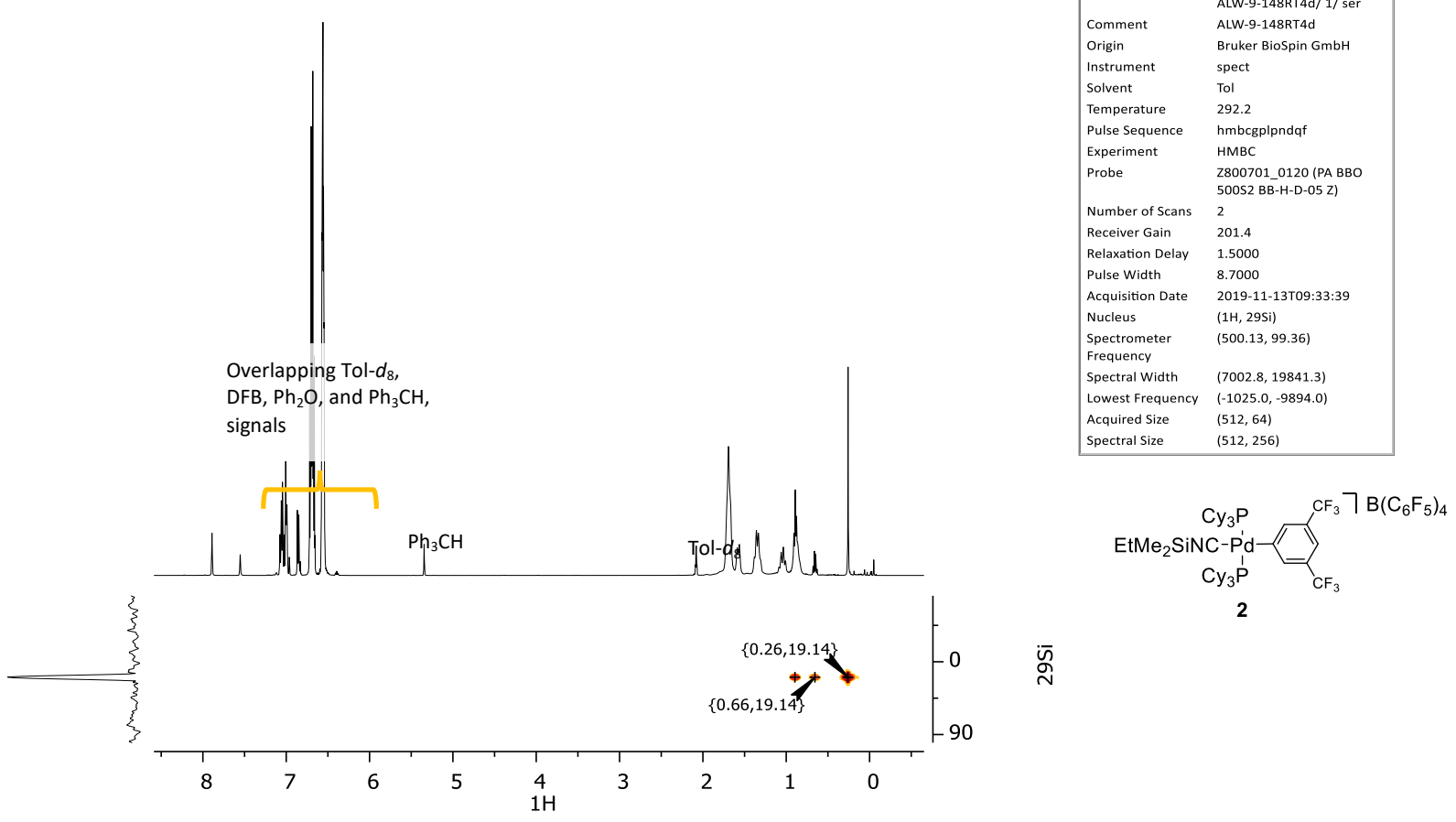

Figure S46: (500 MHz, $99 \mathrm{MHz}){ }^{1} \mathrm{H},{ }^{29} \mathrm{Si}-\mathrm{HMBC}$ NMR spectrum of 2 at room temperature. Solvent: 1:5 DFB:Tol- $d_{8}$. 


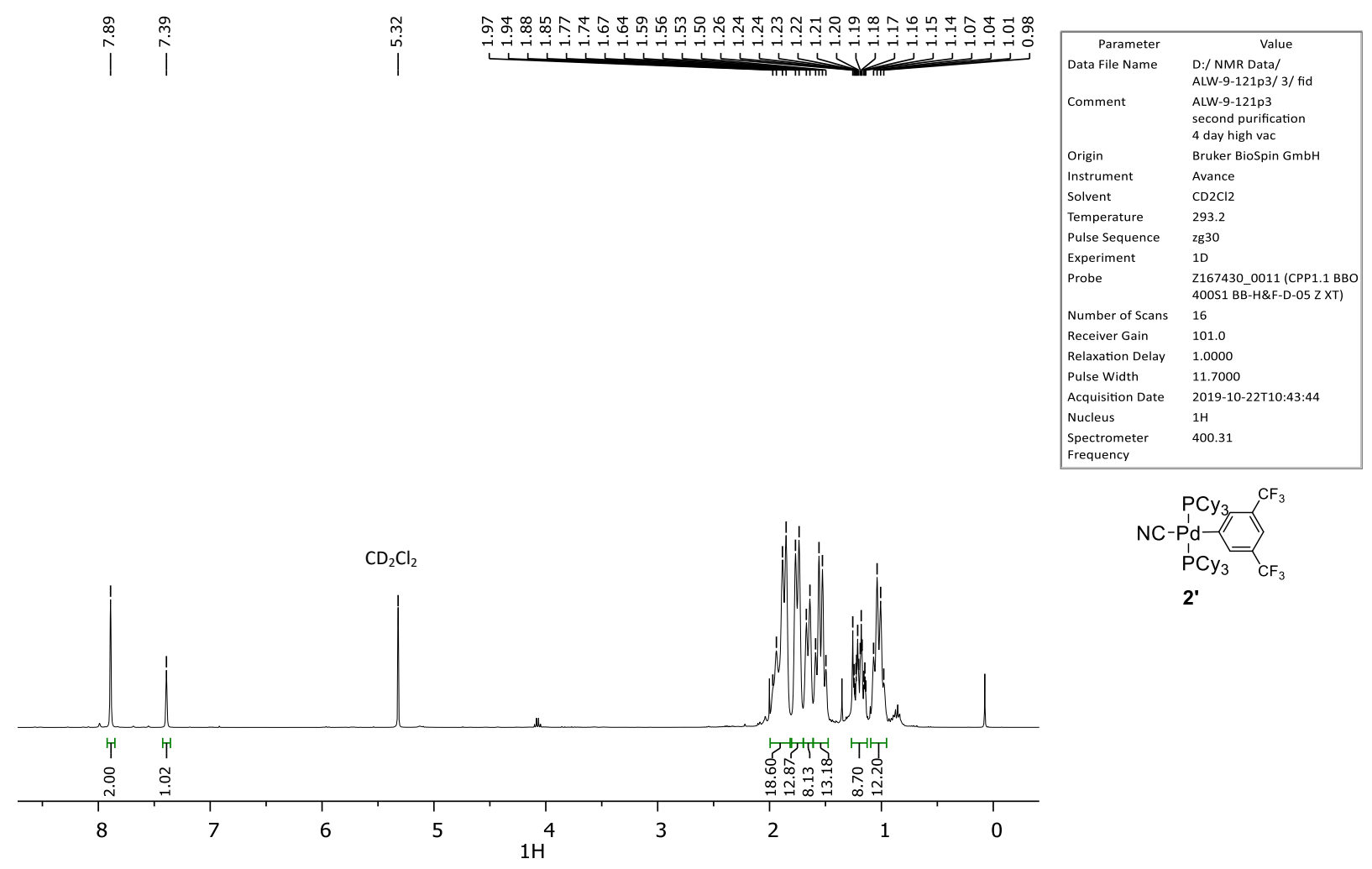

Figure S47: $400 \mathrm{MHz}{ }^{1} \mathrm{H}$ NMR spectrum of 2' at room temperature. Solvent: $\mathrm{CD}_{2} \mathrm{Cl}_{2}$.

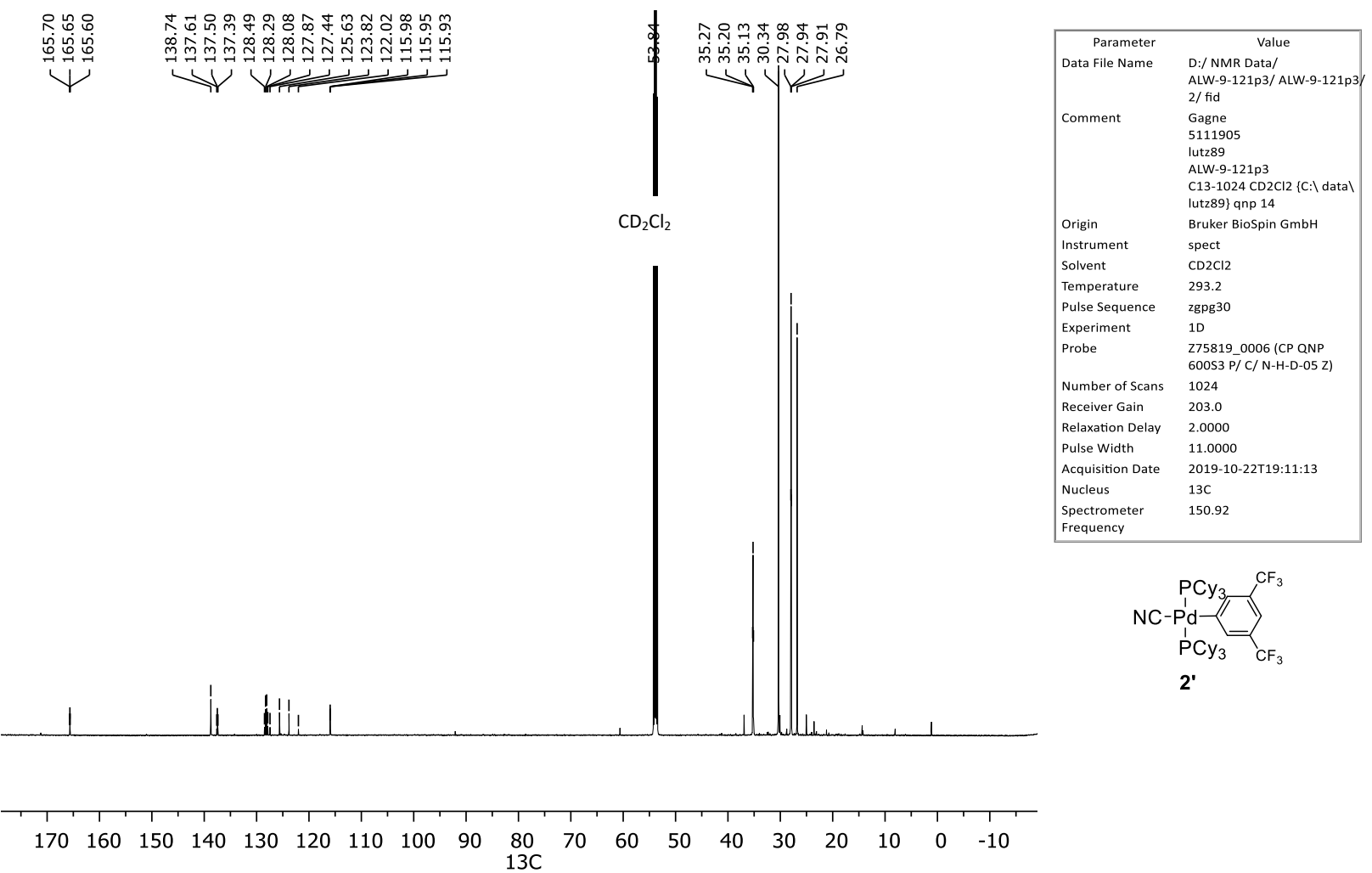

Figure S48: $151 \mathrm{MHz}{ }^{13} \mathrm{C}\left\{{ }^{1} \mathrm{H}\right\}$ NMR spectrum of 2' at room temperature. Solvent: $\mathrm{CD}_{2} \mathrm{Cl}_{2}$. 


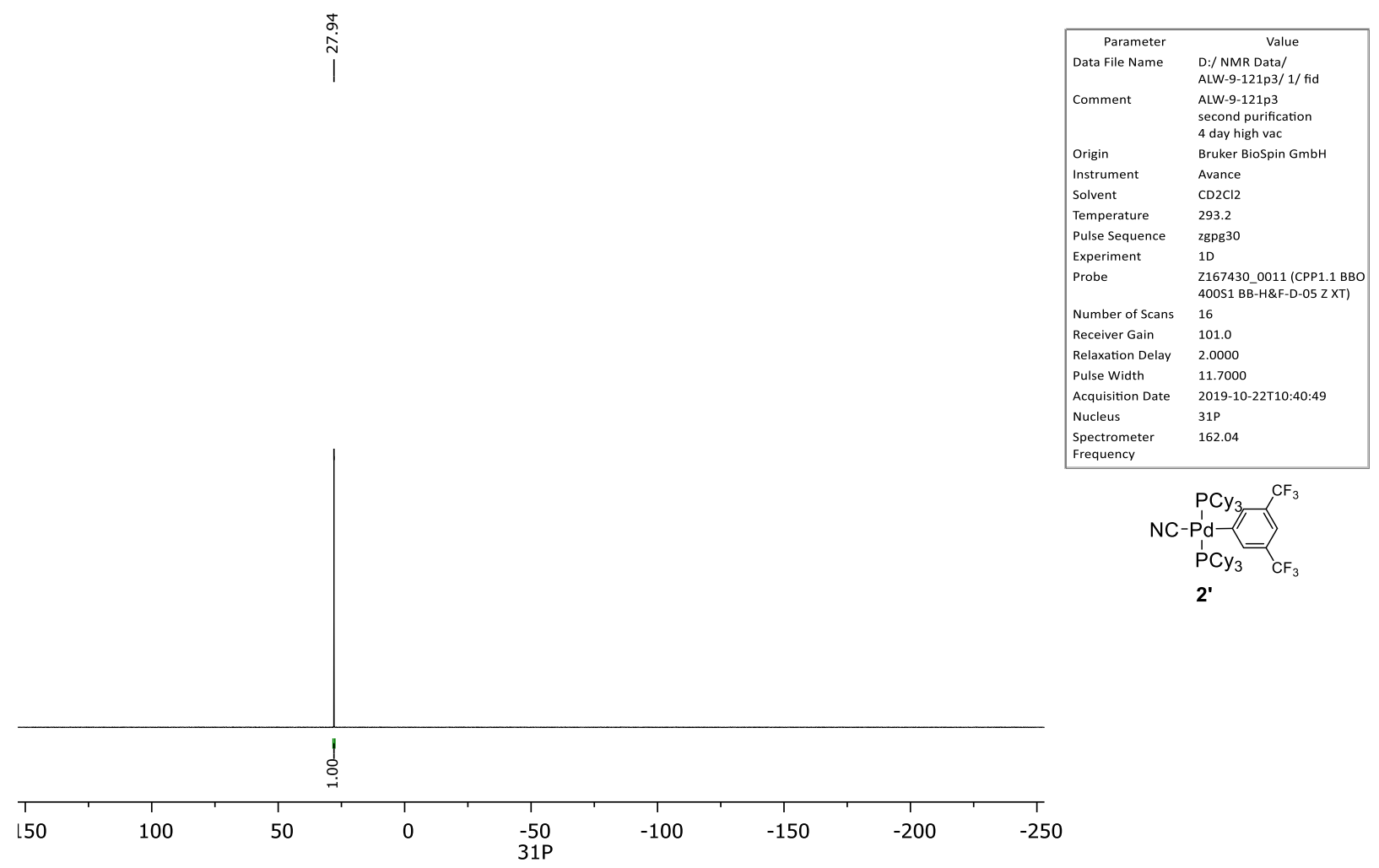

Figure S49: $162 \mathrm{MHz}{ }^{31} \mathrm{P}\left\{{ }^{1} \mathrm{H}\right\}$ NMR spectrum of 2' at room temperature. Solvent: $\mathrm{CD}_{2} \mathrm{Cl}_{2}$.

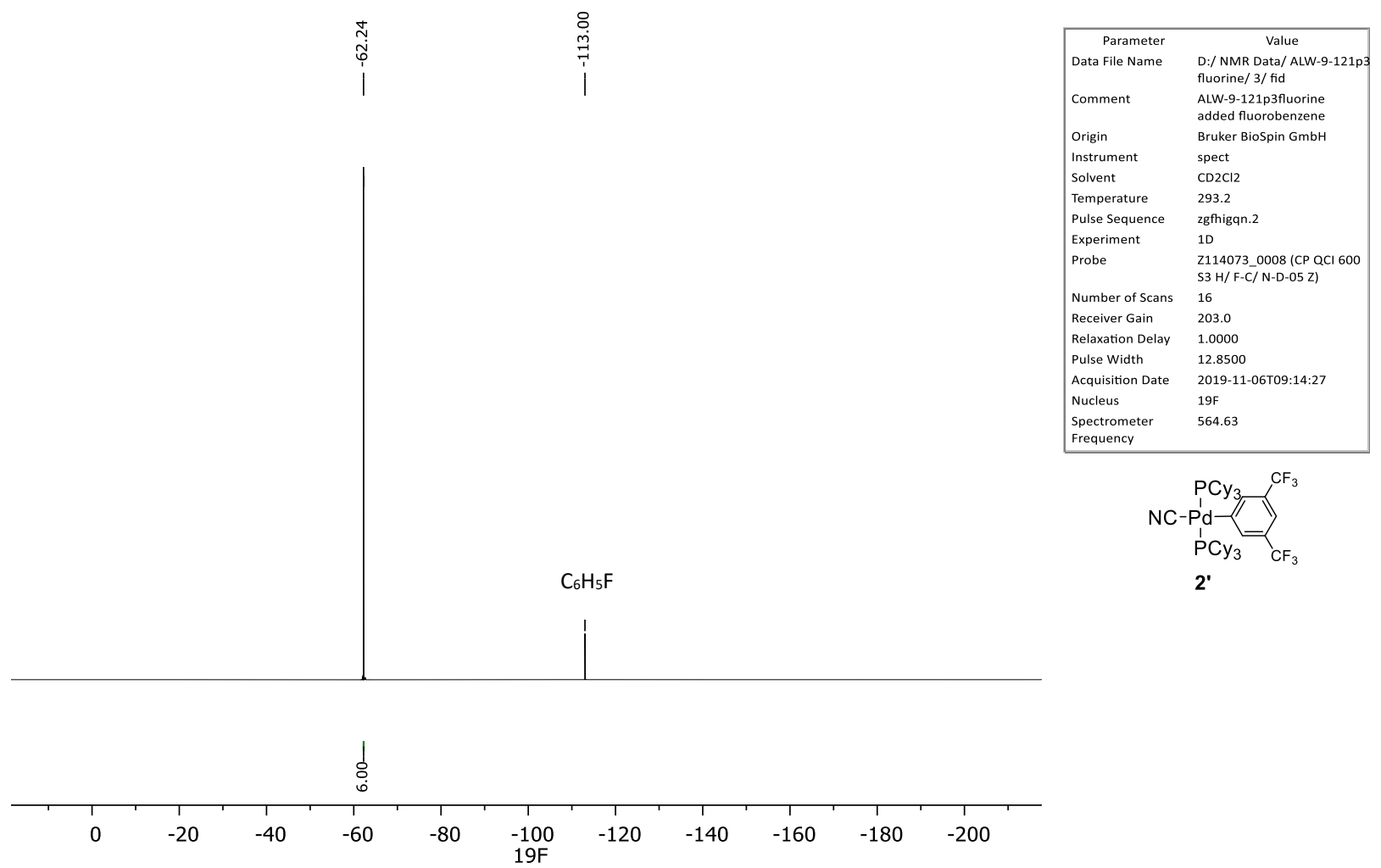

Figure S50: $565 \mathrm{MHz}{ }^{19} \mathrm{~F}\left\{{ }^{1} \mathrm{H}\right\}$ NMR spectrum of 2' at room temperature. Solvent: $\mathrm{CD}_{2} \mathrm{Cl}_{2}$. 


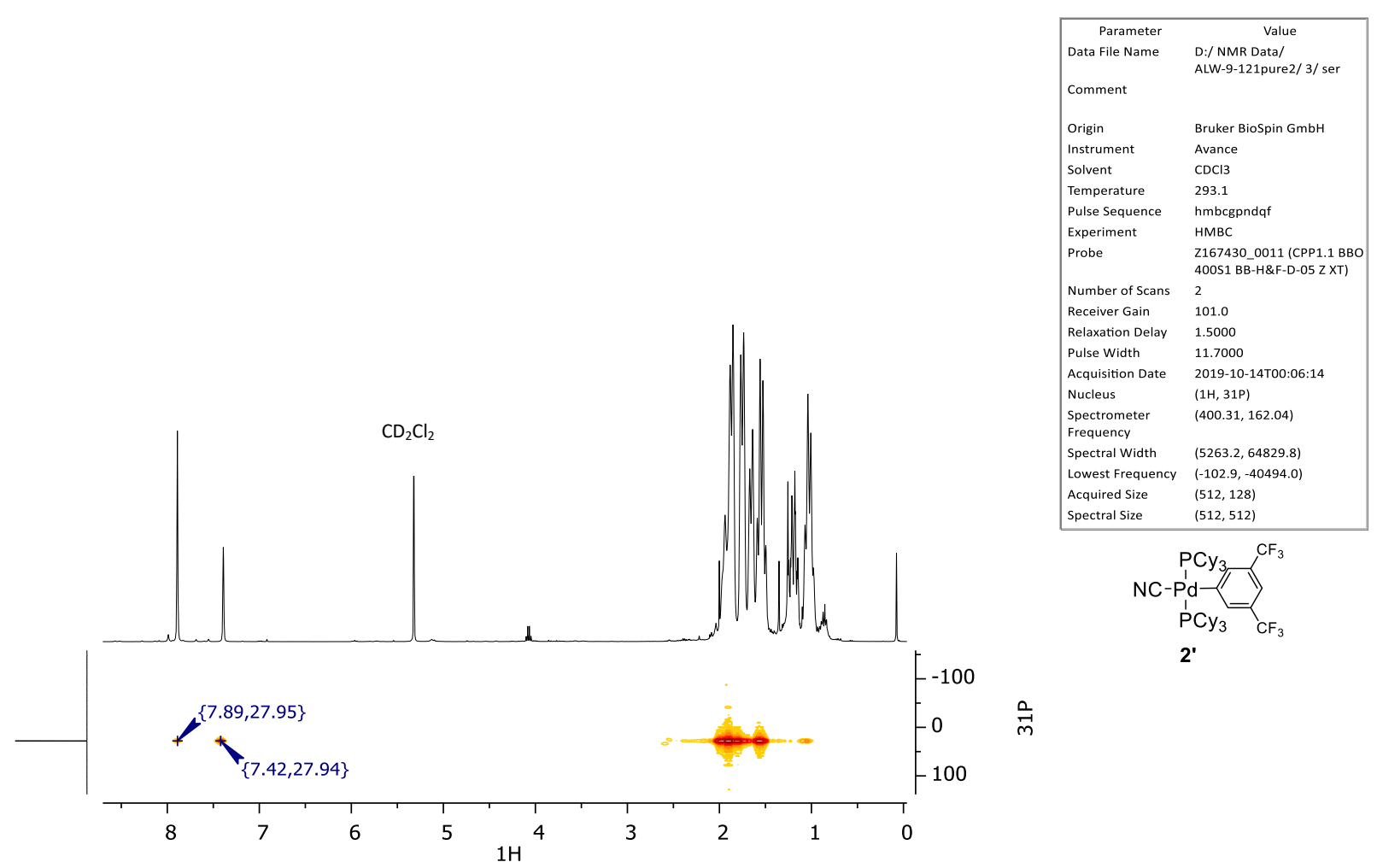

Figure S51: (400 MHz, $162 \mathrm{MHz}$ ) ${ }^{1} \mathrm{H},{ }^{31} \mathrm{P}-\mathrm{HMBC}$ NMR spectrum of 2' at room temperature. Solvent: $\mathrm{CD}_{2} \mathrm{Cl}_{2}$. 


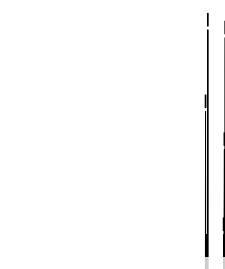

Overlapping Tol- $d_{8}$, $\mathrm{DFB}, \mathrm{Ph}_{2} \mathrm{O}, \mathrm{Ph}_{3} \mathrm{CH}$, and 3 signals
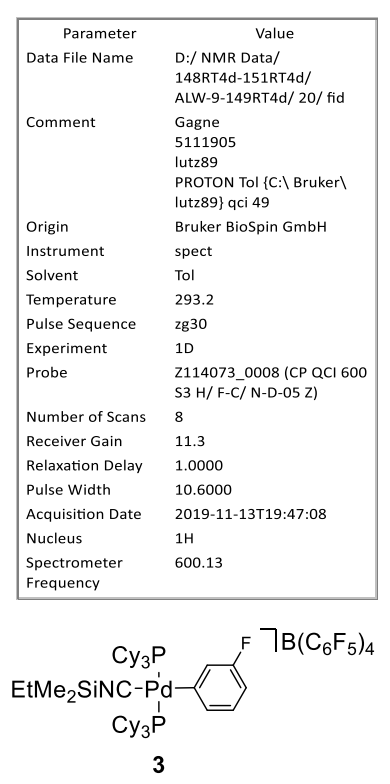

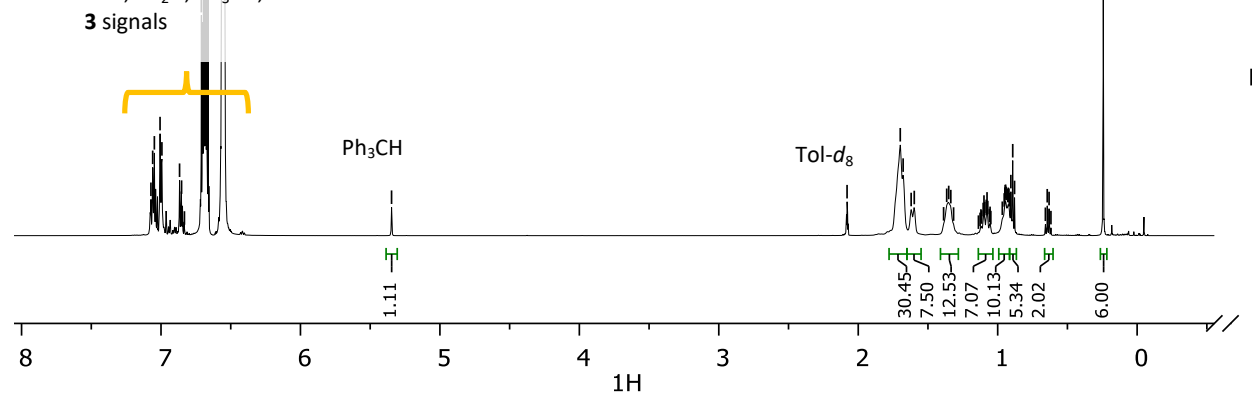

Figure S52: $600 \mathrm{MHz}{ }^{1} \mathrm{H}$ NMR spectrum of 3 at room temperature. Solvent: 1:5 DFB:Tol- $d_{8}$.

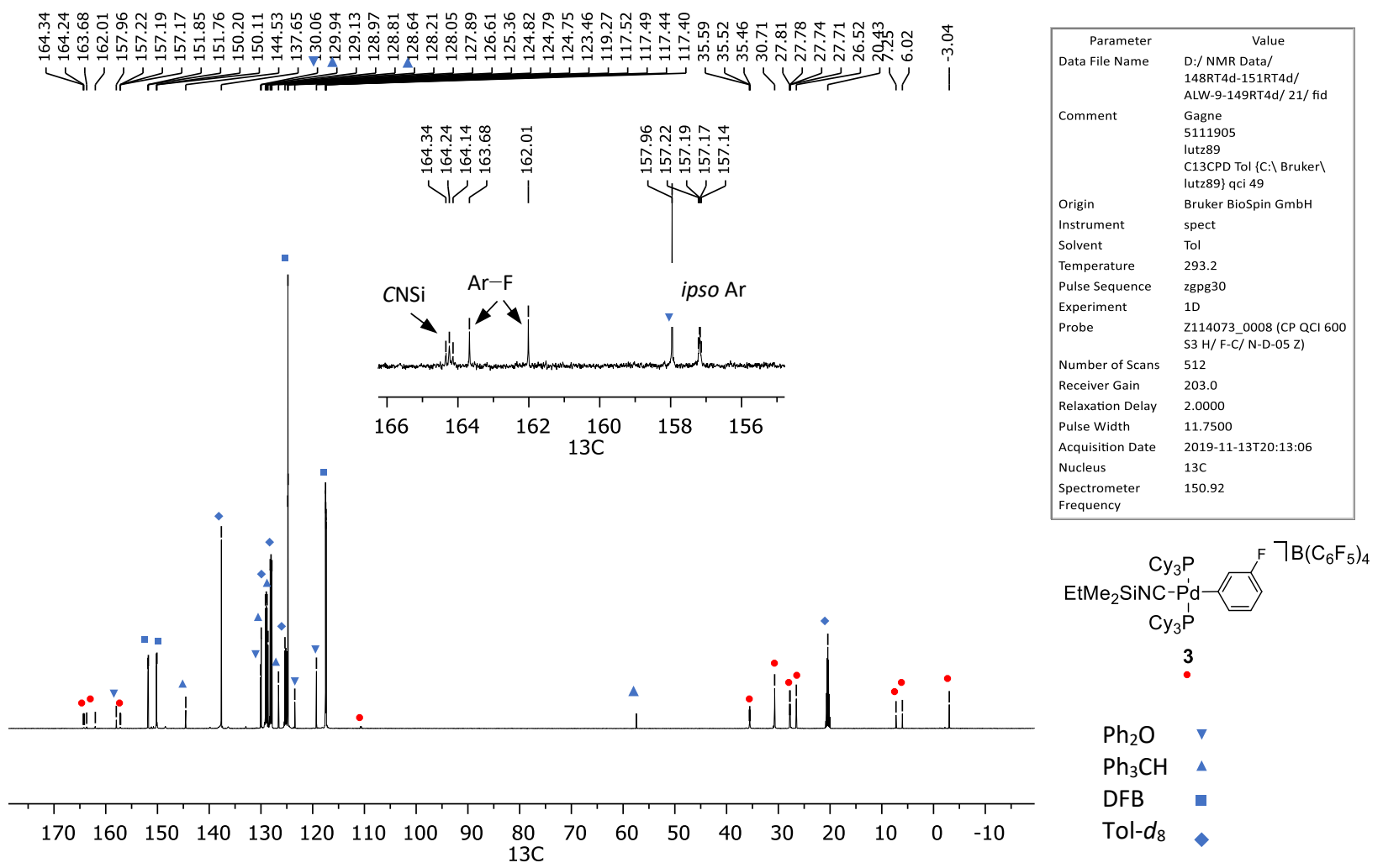

Figure S53: $151 \mathrm{MHz}{ }^{13} \mathrm{C}\left\{{ }^{1} \mathrm{H}\right\}$ NMR spectrum of 3 at room temperature. Solvent: 1:5 DFB:Tol- $d_{8}$. 


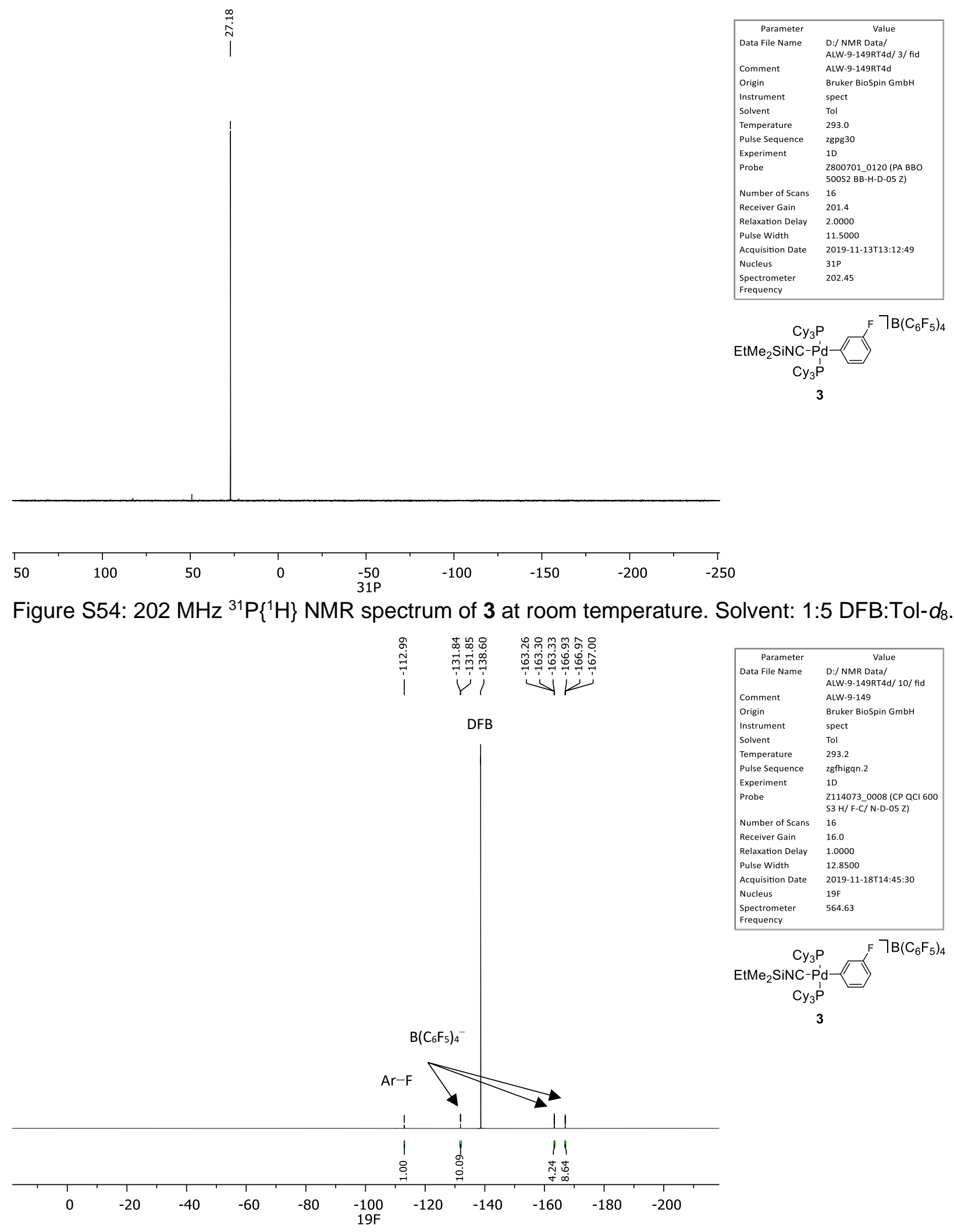

Figure S55: $565 \mathrm{MHz}{ }^{19} \mathrm{~F}\left\{{ }^{1} \mathrm{H}\right\}$ NMR spectrum of 3 at room temperature. Solvent: 1:5 DFB:Tol- $d_{8}$. 


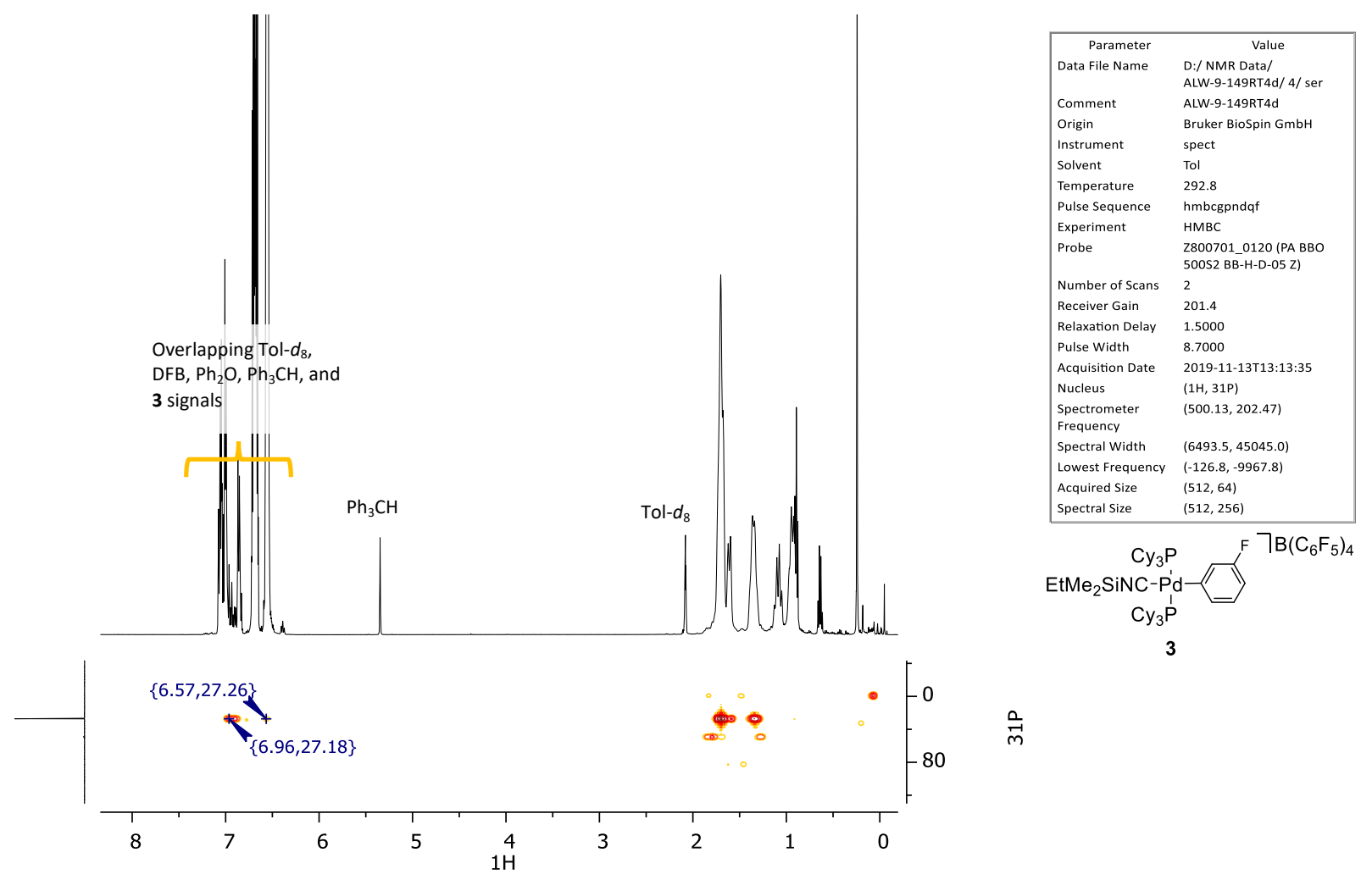

Figure S56: (500 MHz, $202 \mathrm{MHz}){ }^{1} \mathrm{H},{ }^{31} \mathrm{P}-\mathrm{HMBC}$ NMR spectrum of 3 at room temperature. Solvent: 1:5 DFB:Tol- $d_{8}$.

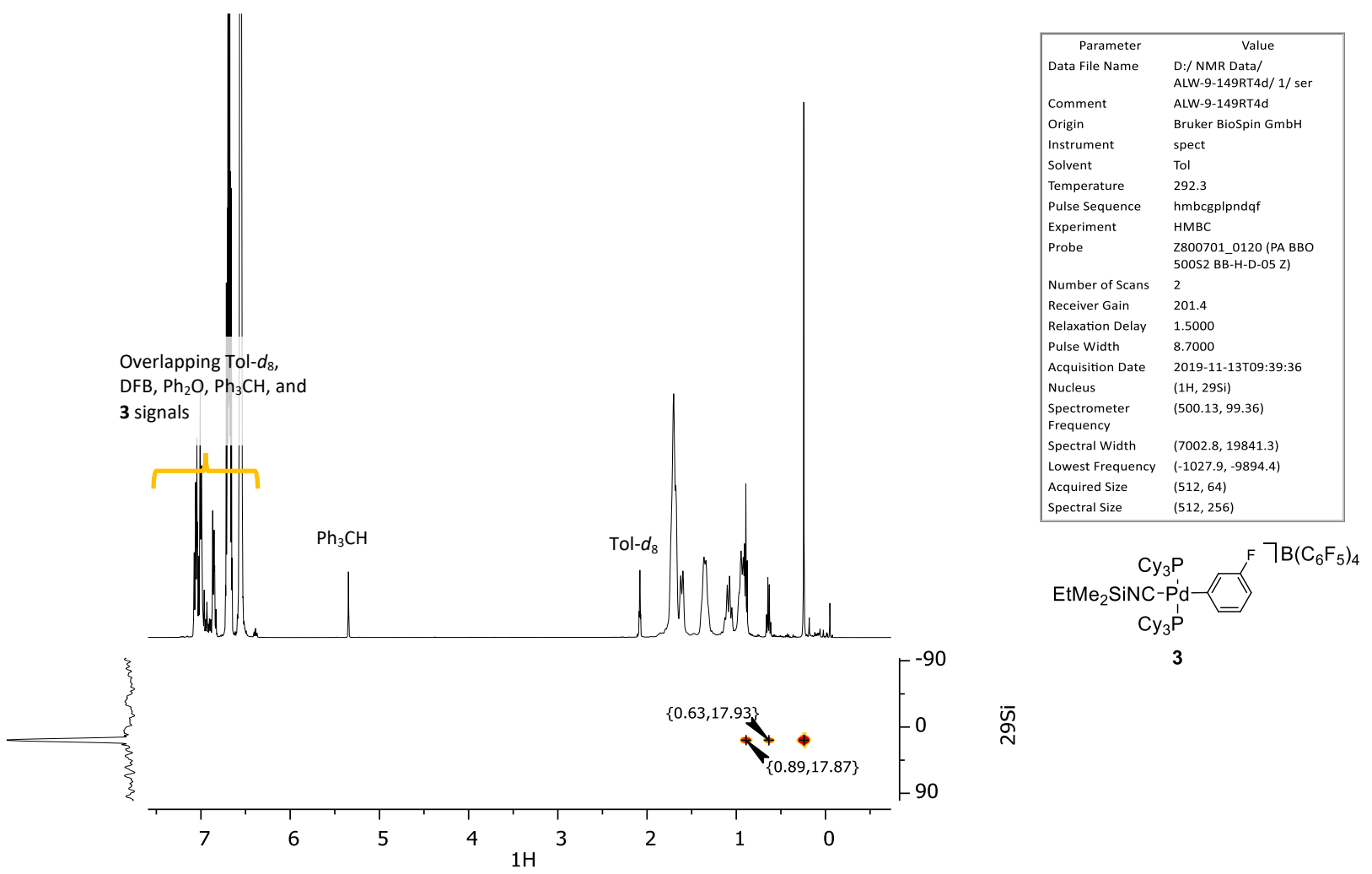

Figure S57: (500 MHz, $99 \mathrm{MHz}){ }^{1} \mathrm{H},{ }^{29} \mathrm{Si}-\mathrm{HMBC}$ NMR spectrum of 3 at room temperature. Solvent: 1:5 DFB:Tol- $d_{8}$. 


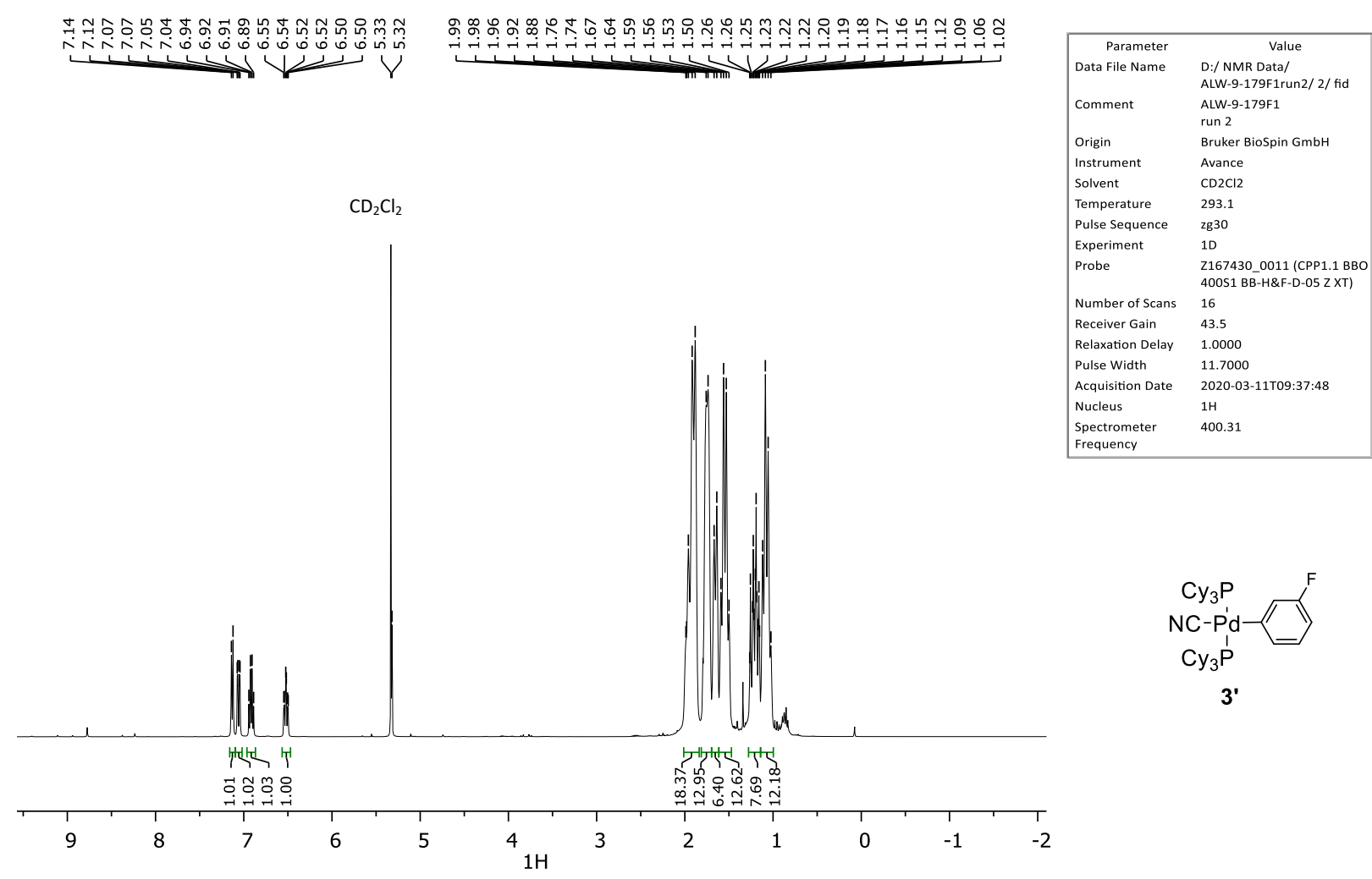

Figure S58: $400 \mathrm{MHz}{ }^{1} \mathrm{H}$ NMR spectrum of 3' at room temperature. Solvent: $\mathrm{CD}_{2} \mathrm{Cl}_{2}$.

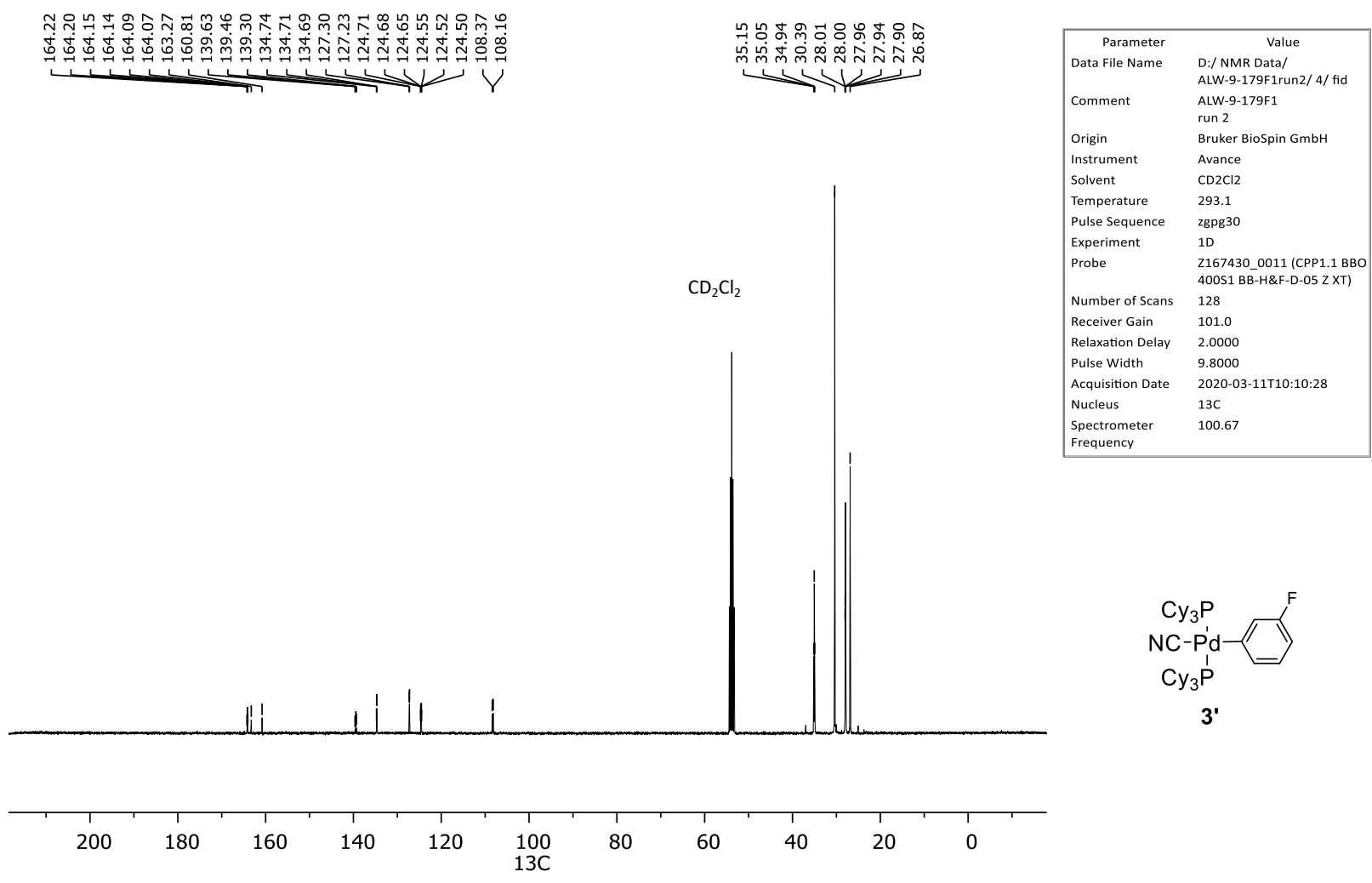

Figure S59: $101 \mathrm{MHz}{ }^{13} \mathrm{C}\left\{{ }^{1} \mathrm{H}\right\}$ NMR spectrum of 3' at room temperature. Solvent: $\mathrm{CD}_{2} \mathrm{Cl}_{2}$. 


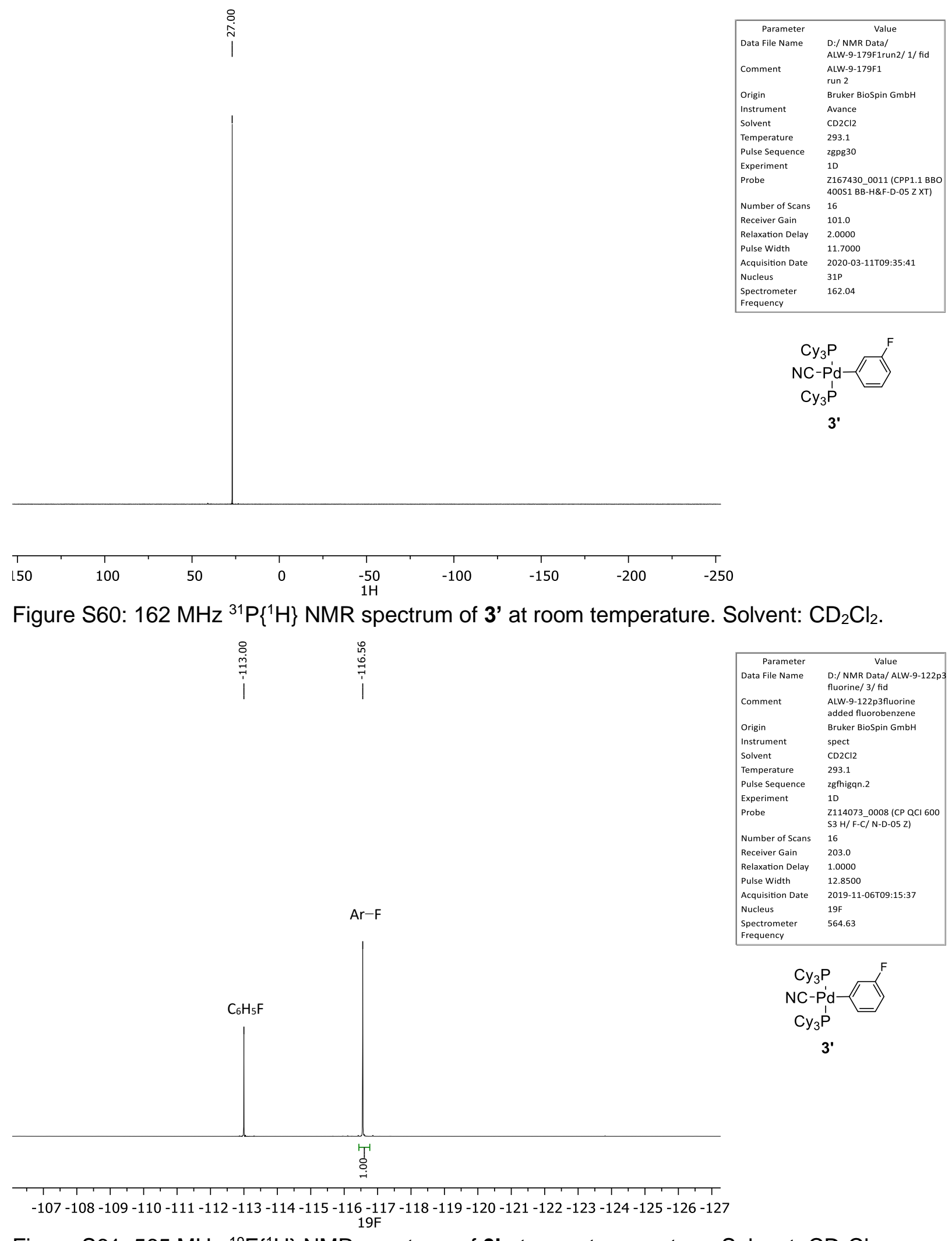

Figure $\mathrm{S} 61: 565 \mathrm{MHz}{ }^{19} \mathrm{~F}\left\{{ }^{1} \mathrm{H}\right\} \mathrm{NMR}$ spectrum of $3^{\prime}$ at room temperature. Solvent: $\mathrm{CD}_{2} \mathrm{Cl}_{2}$. 


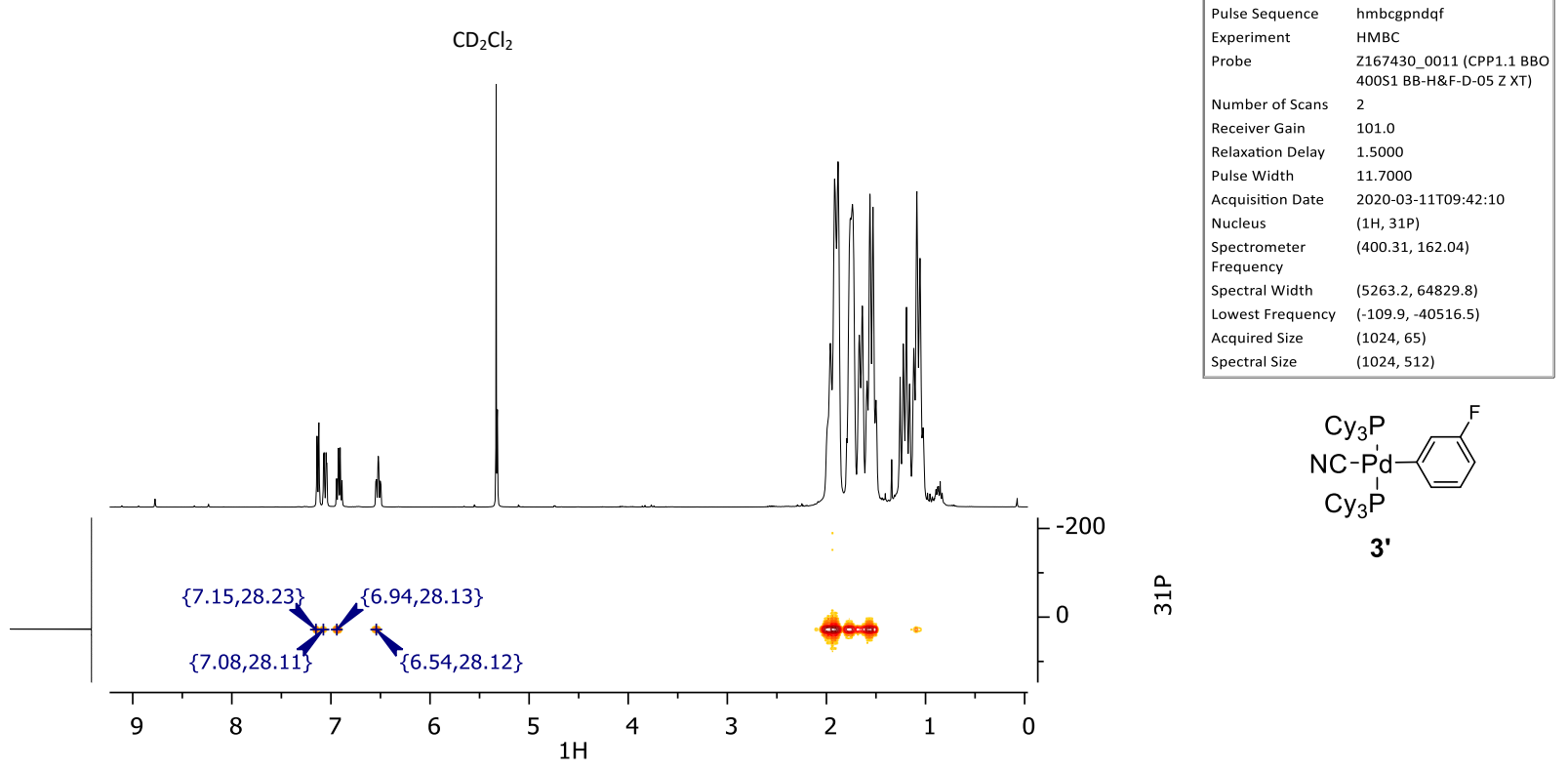

Figure S62: (400 MHz, $162 \mathrm{MHz}){ }^{1} \mathrm{H},{ }^{31} \mathrm{P}-\mathrm{HMBC}$ NMR spectrum of 3' at room temperature. Solvent $\mathrm{CD}_{2} \mathrm{Cl}_{2}$. 


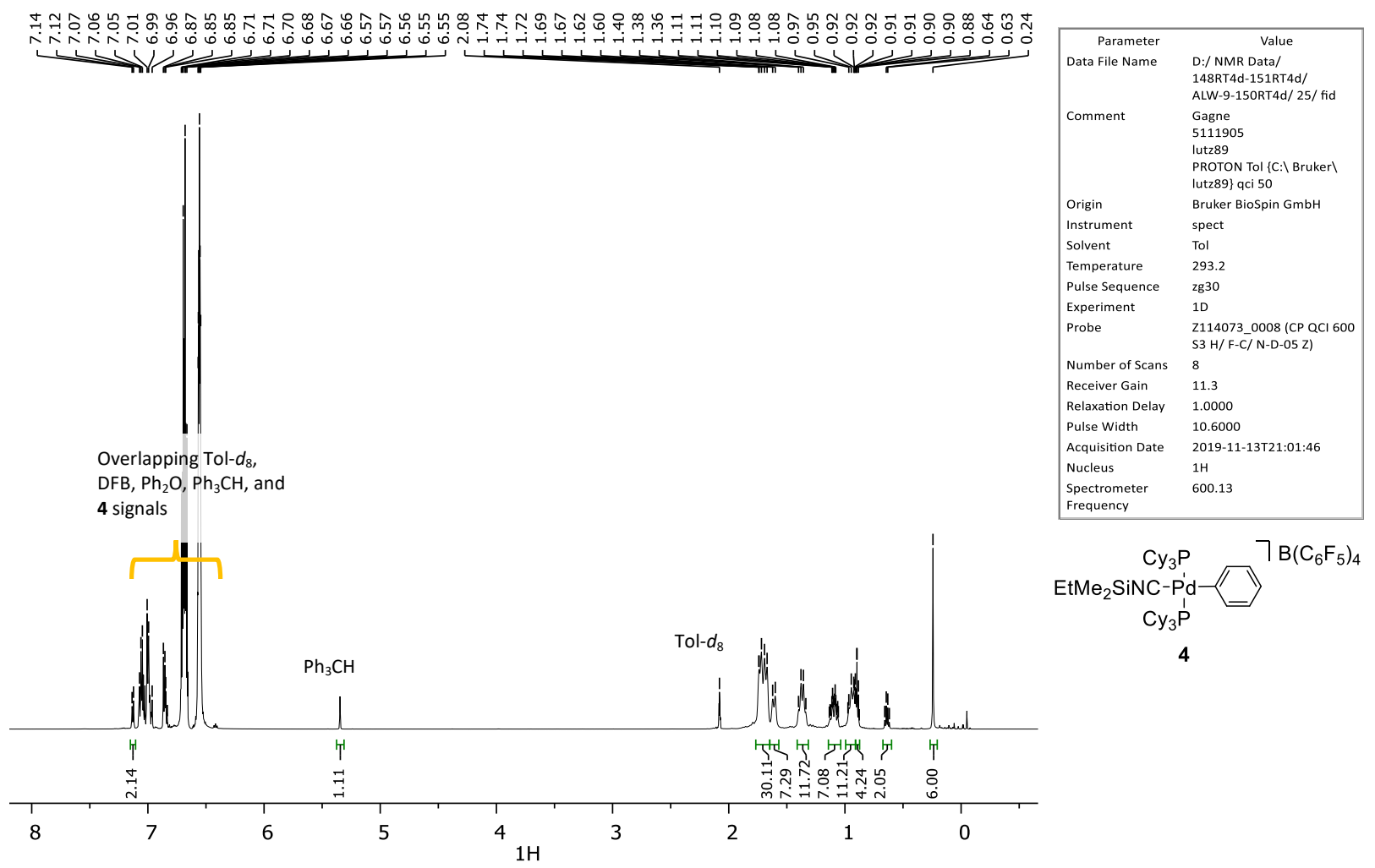

Figure S63: $600 \mathrm{MHz}{ }^{1} \mathrm{H}$ NMR spectrum of 4 at room temperature. Solvent: 1:5 DFB:Tol- $d_{8}$.

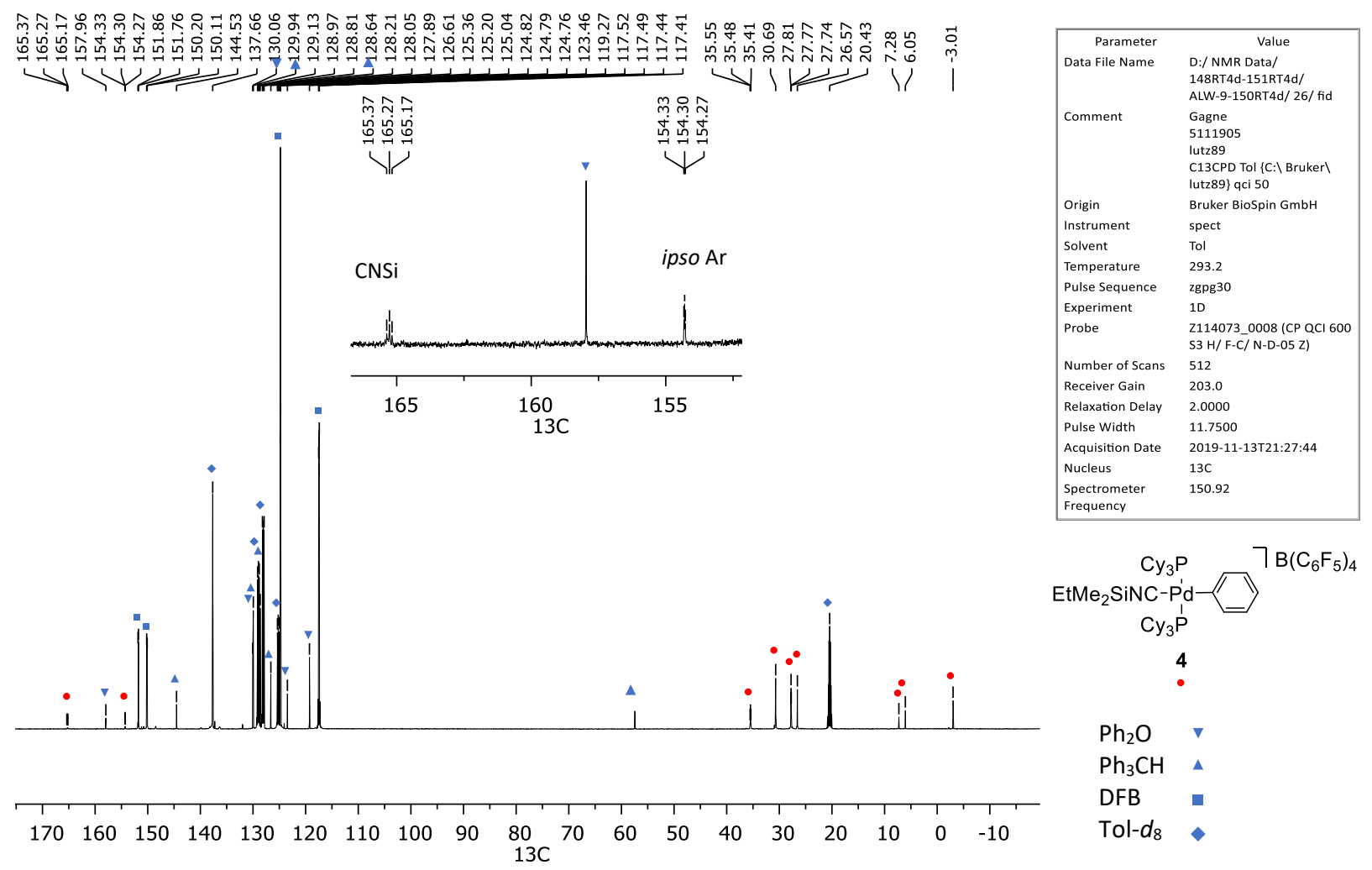

Figure S64: $151 \mathrm{MHz}{ }^{13} \mathrm{C}\left\{{ }^{1} \mathrm{H}\right\}$ NMR spectrum of 4 at room temperature. Solvent: 1:5 DFB:Tol- $d_{8}$. 


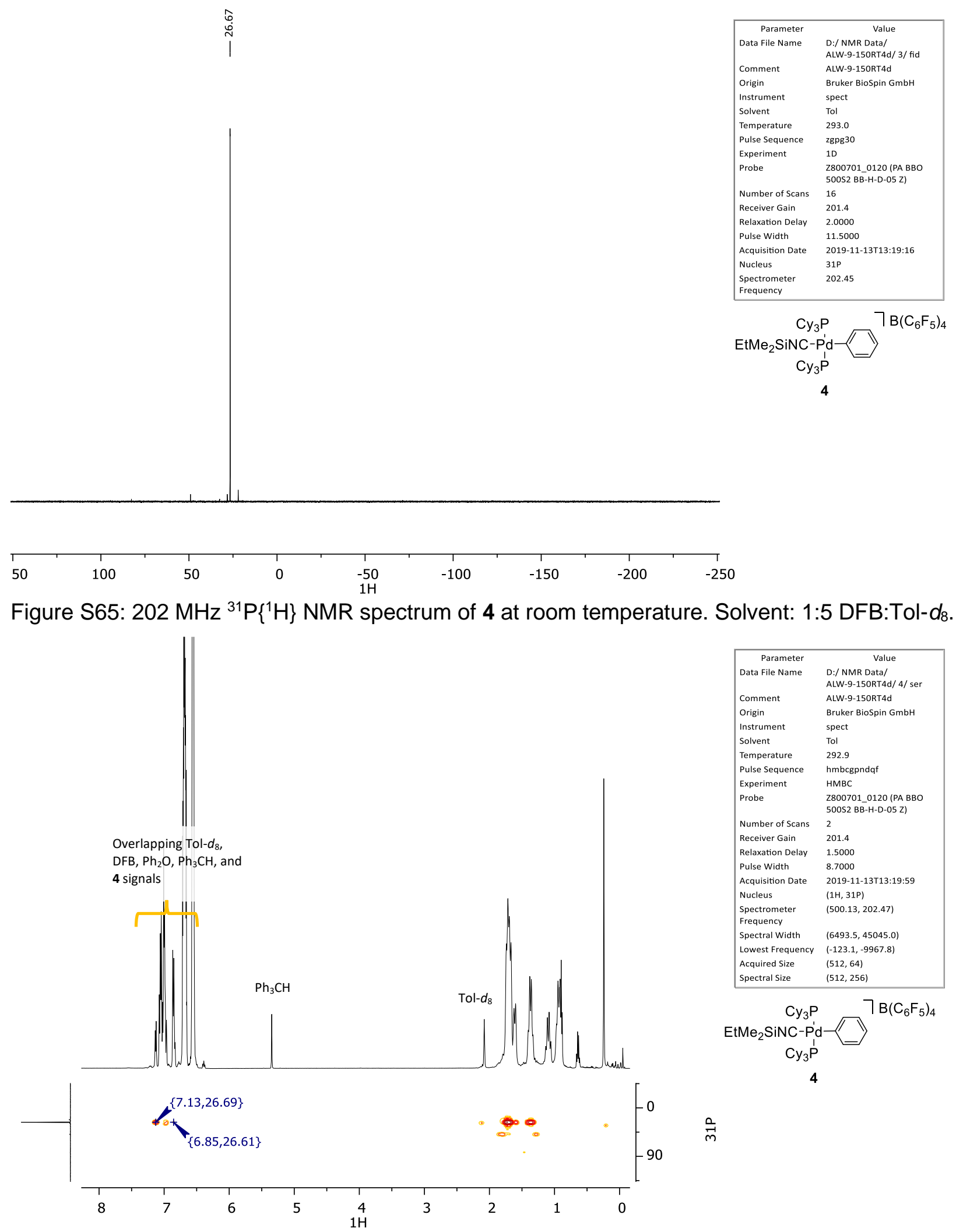

Figure S66: (500 MHz, $202 \mathrm{MHz}){ }^{1} \mathrm{H},{ }^{31} \mathrm{P}-\mathrm{HMBC}$ NMR spectrum for 4 at room temperature. Solvent: 1:5 DFB:Tol- $d_{8}$. 


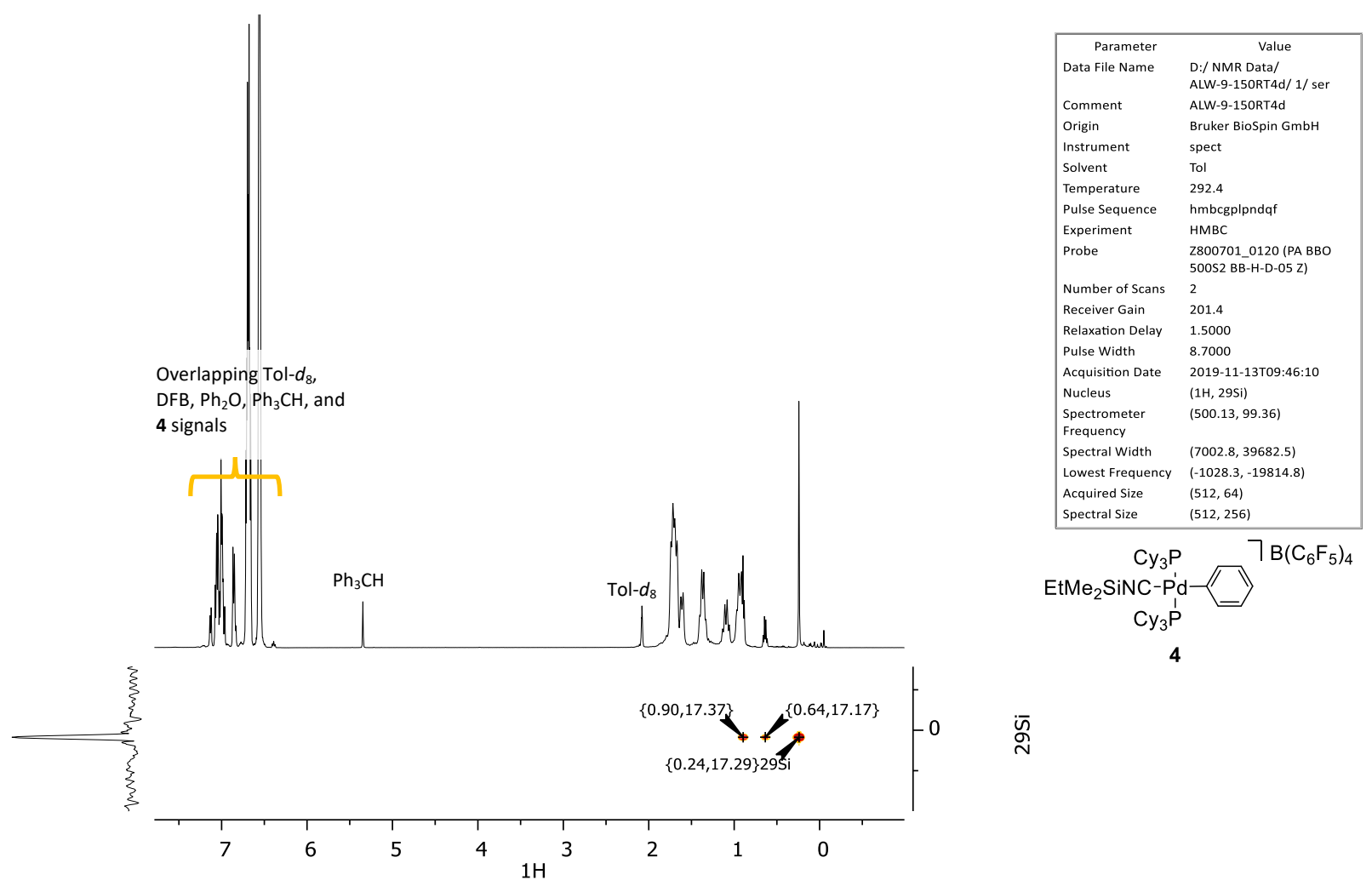

Figure S67: (500 MHz, $99 \mathrm{MHz}){ }^{1} \mathrm{H},{ }^{29} \mathrm{Si}-\mathrm{HMBC}$ NMR spectrum for 4 at room temperature. Solvent: 1:5 DFB:Tol-d8. 


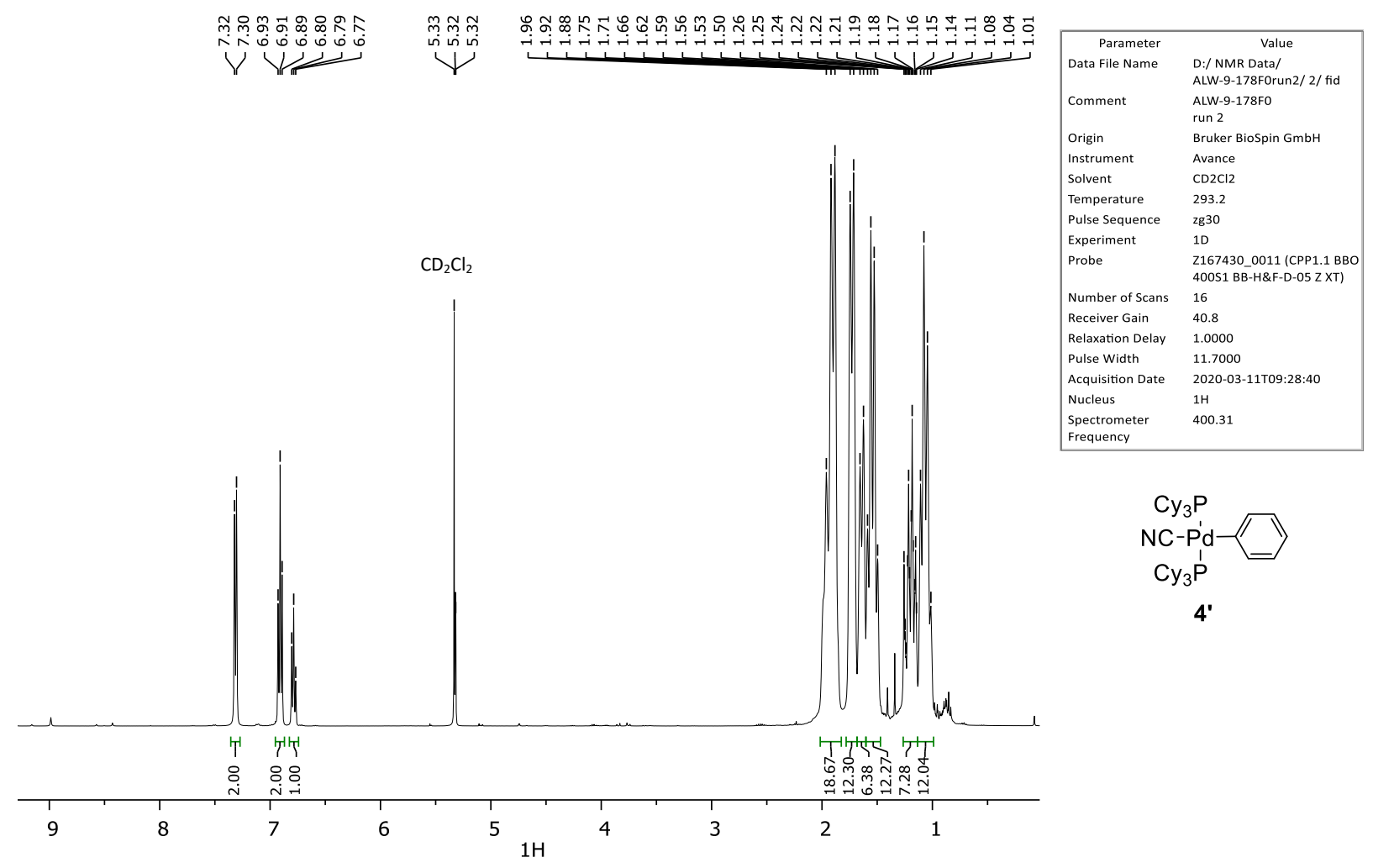

Figure S68: $400 \mathrm{MHz}{ }^{1} \mathrm{H}$ NMR spectrum of 4' at room temperature. Solvent: $\mathrm{CD}_{2} \mathrm{Cl}_{2}$.

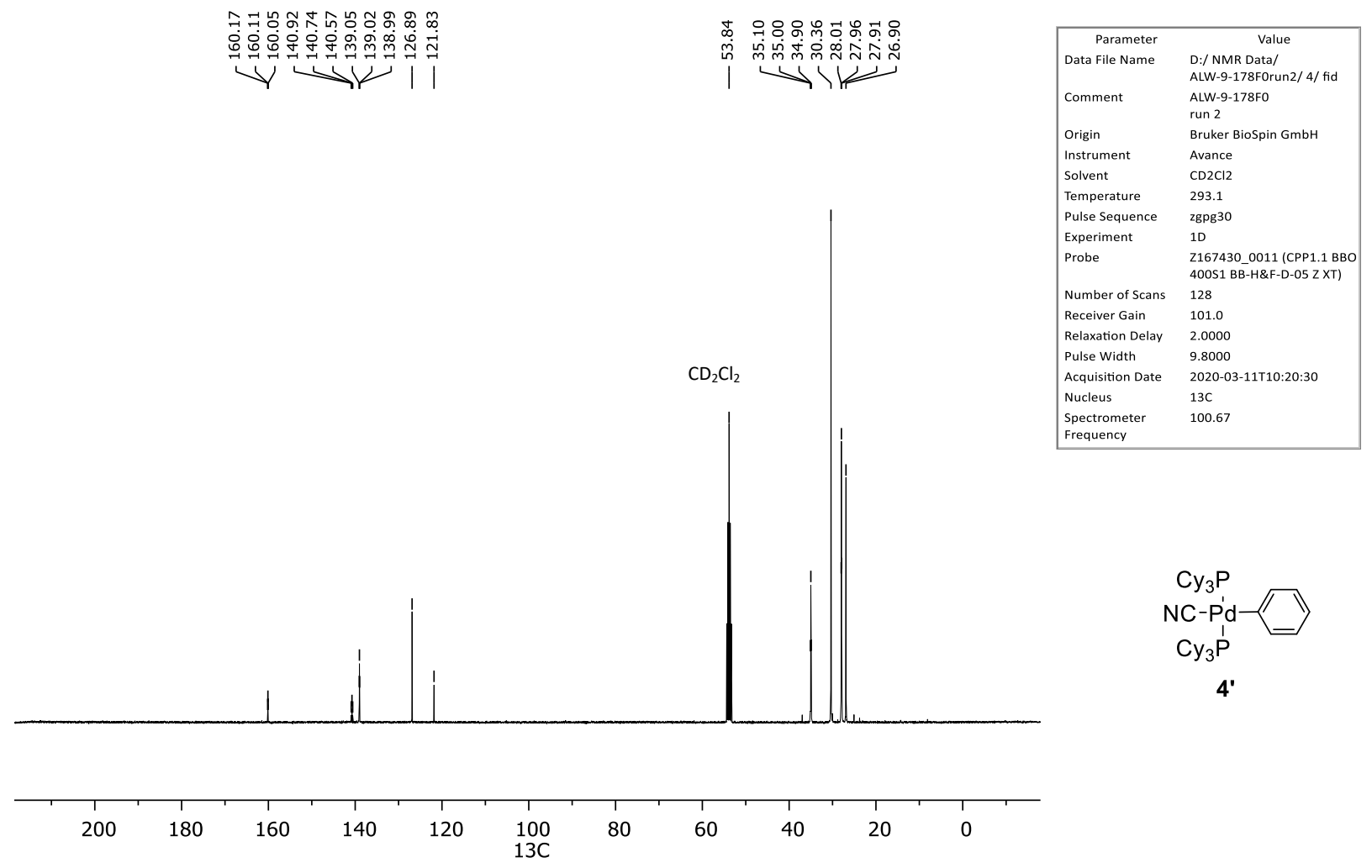

Figure S69: $101 \mathrm{MHz}{ }^{13} \mathrm{C}\left\{{ }^{1} \mathrm{H}\right\}$ NMR spectrum of 4' at room temperature. Solvent: $\mathrm{CD}_{2} \mathrm{Cl}_{2}$. 


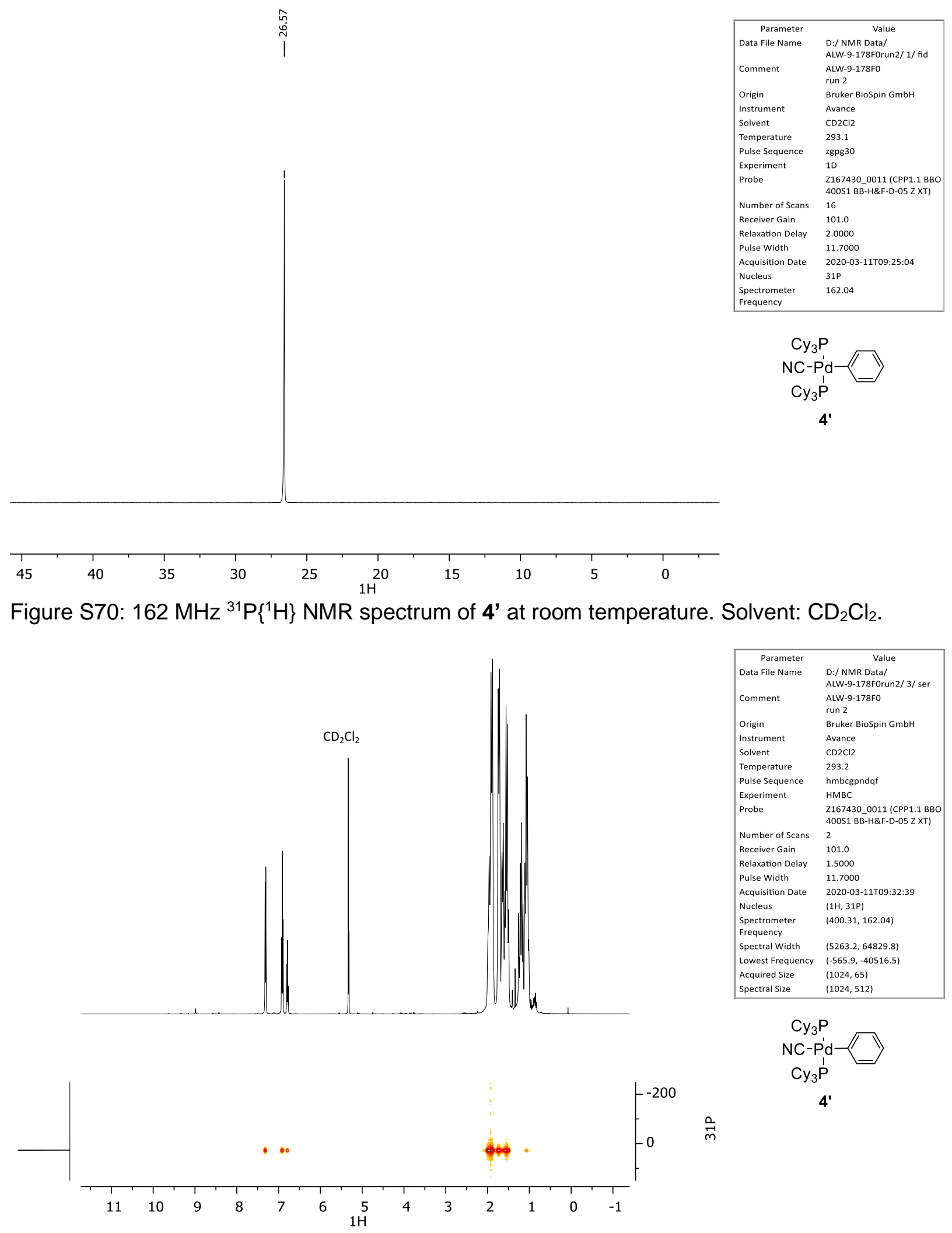

Figure S71: (400 MHz, $162 \mathrm{MHz}$ ) ${ }^{1} \mathrm{H},{ }^{31} \mathrm{P}-\mathrm{HMBC}$ NMR spectrum of 4' at room temperature. Solvent: $\mathrm{CD}_{2} \mathrm{Cl}_{2}$. 


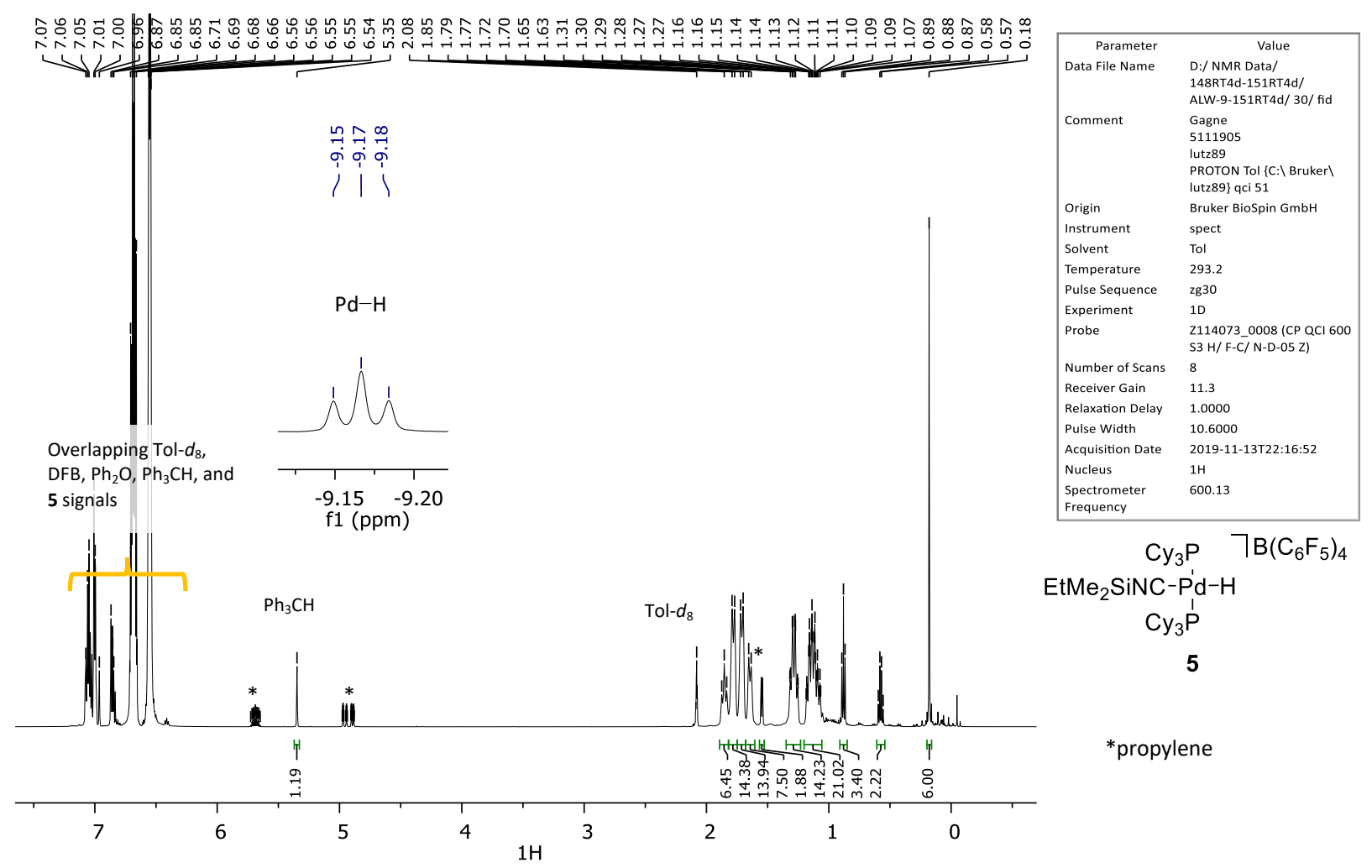

Figure S72: $600 \mathrm{MHz}{ }^{1} \mathrm{H}$ NMR spectrum of 5 at room temperature. Solvent: 1:5 DFB:Tol- $d_{8}$.

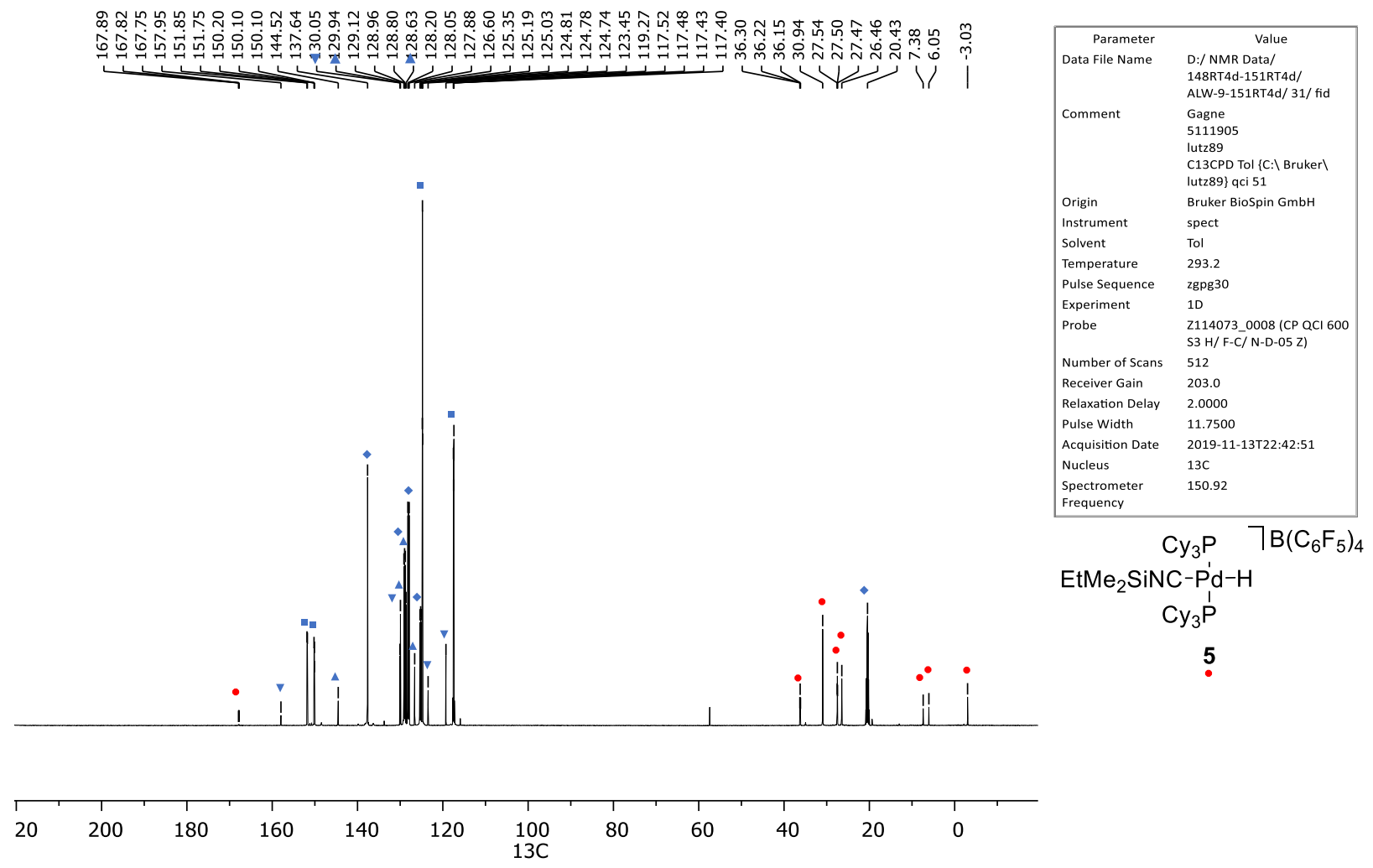

Figure S73: $151 \mathrm{MHz}{ }^{13} \mathrm{C}\left\{{ }^{1} \mathrm{H}\right\}$ NMR spectrum of 5 at room temperature. Solvent: 1:5 DFB:Tol- $d_{8}$. 


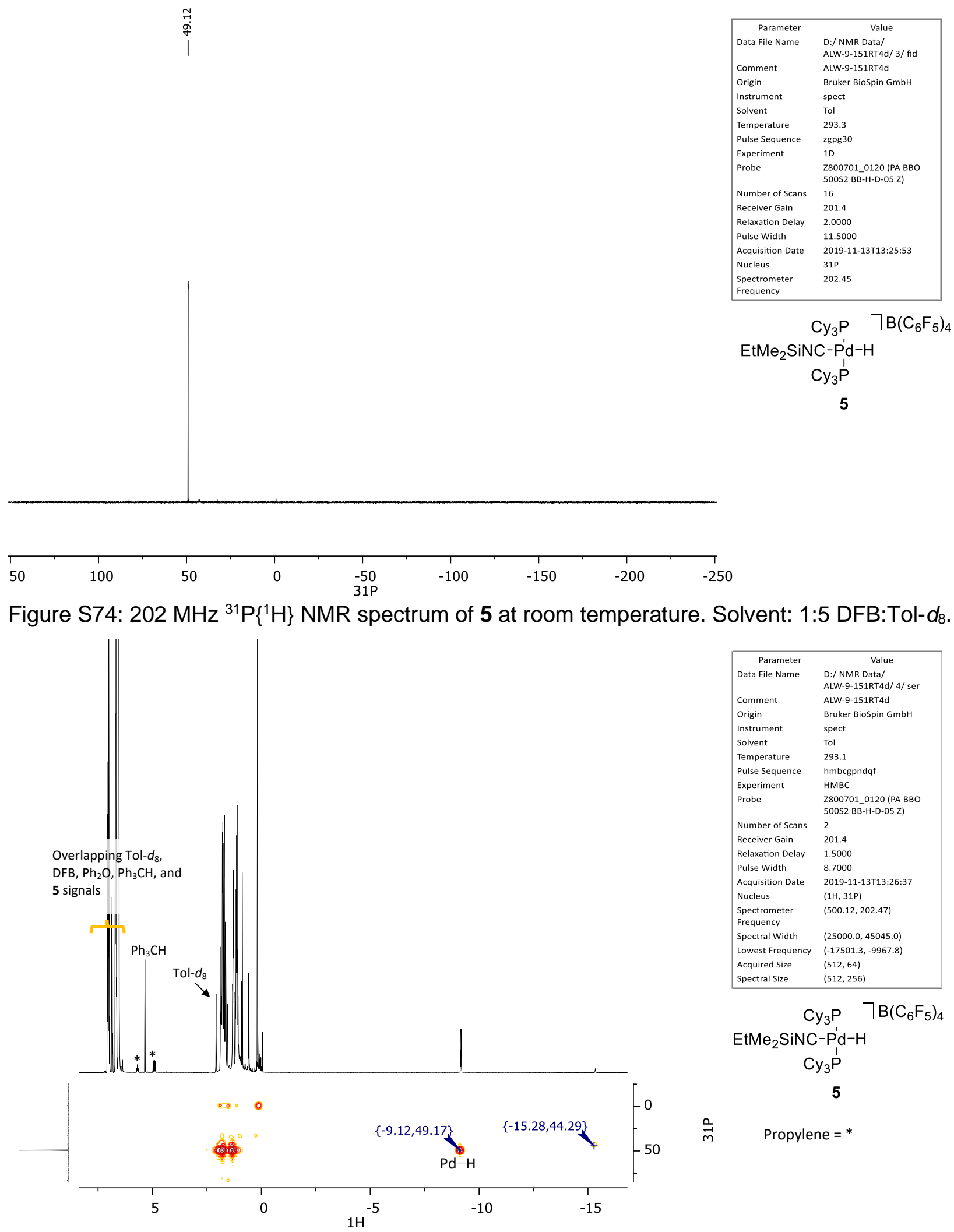

Figure S75: ( $500 \mathrm{MHz}, 202 \mathrm{MHz}){ }^{1} \mathrm{H},{ }^{31} \mathrm{P}-\mathrm{HMBC}$ NMR spectrum of 5 at room temperature. Solvent: 1:5 DFB:Tol- $d_{8}$. 


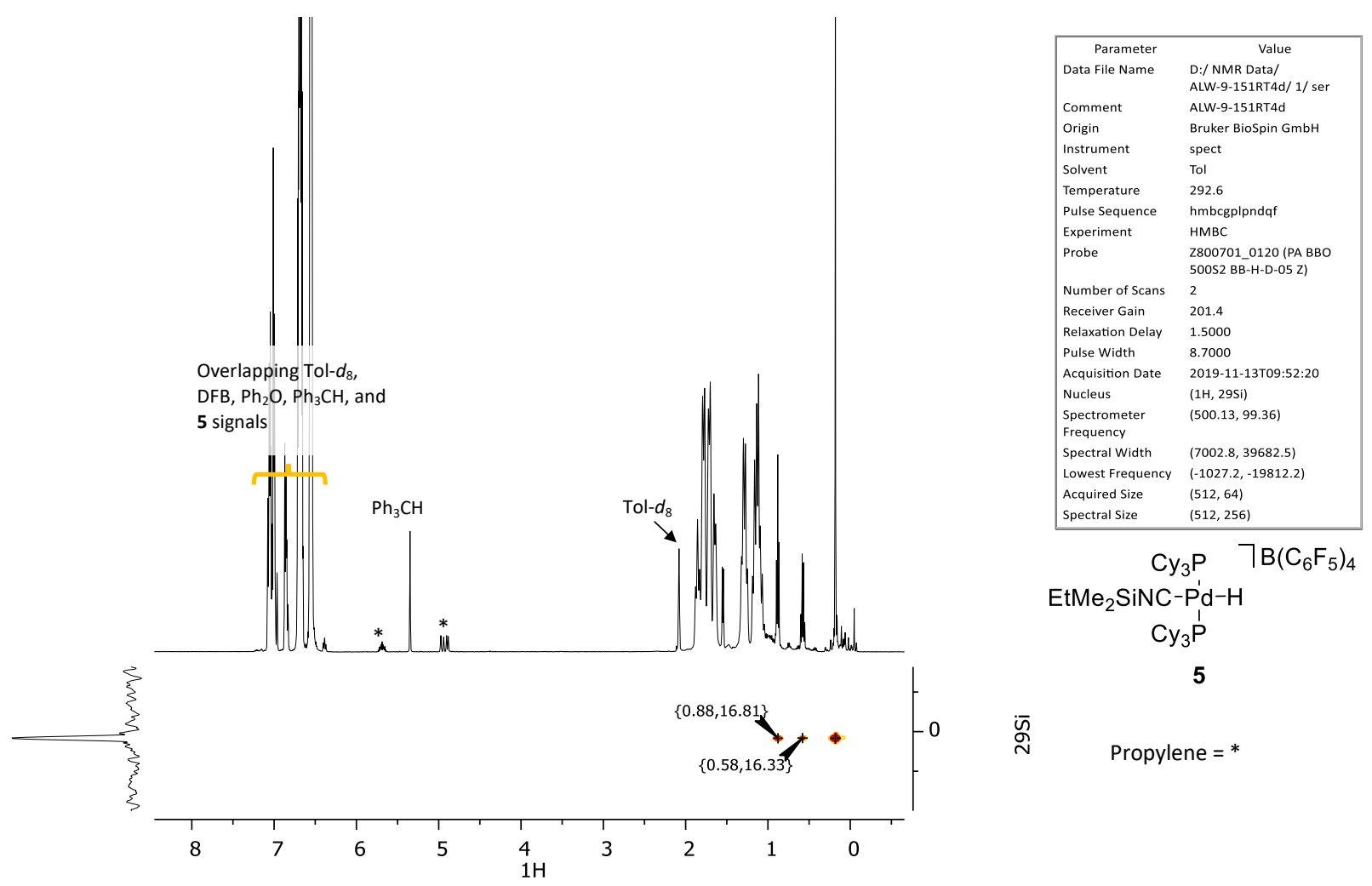

Figure S76: (500 MHz, $99 \mathrm{MHz}){ }^{1} \mathrm{H},{ }^{29} \mathrm{Si}-\mathrm{HMBC}$ NMR spectrum of 5 at room temperature. Solvent: 1:5 DFB:Tol- $d_{8}$. 


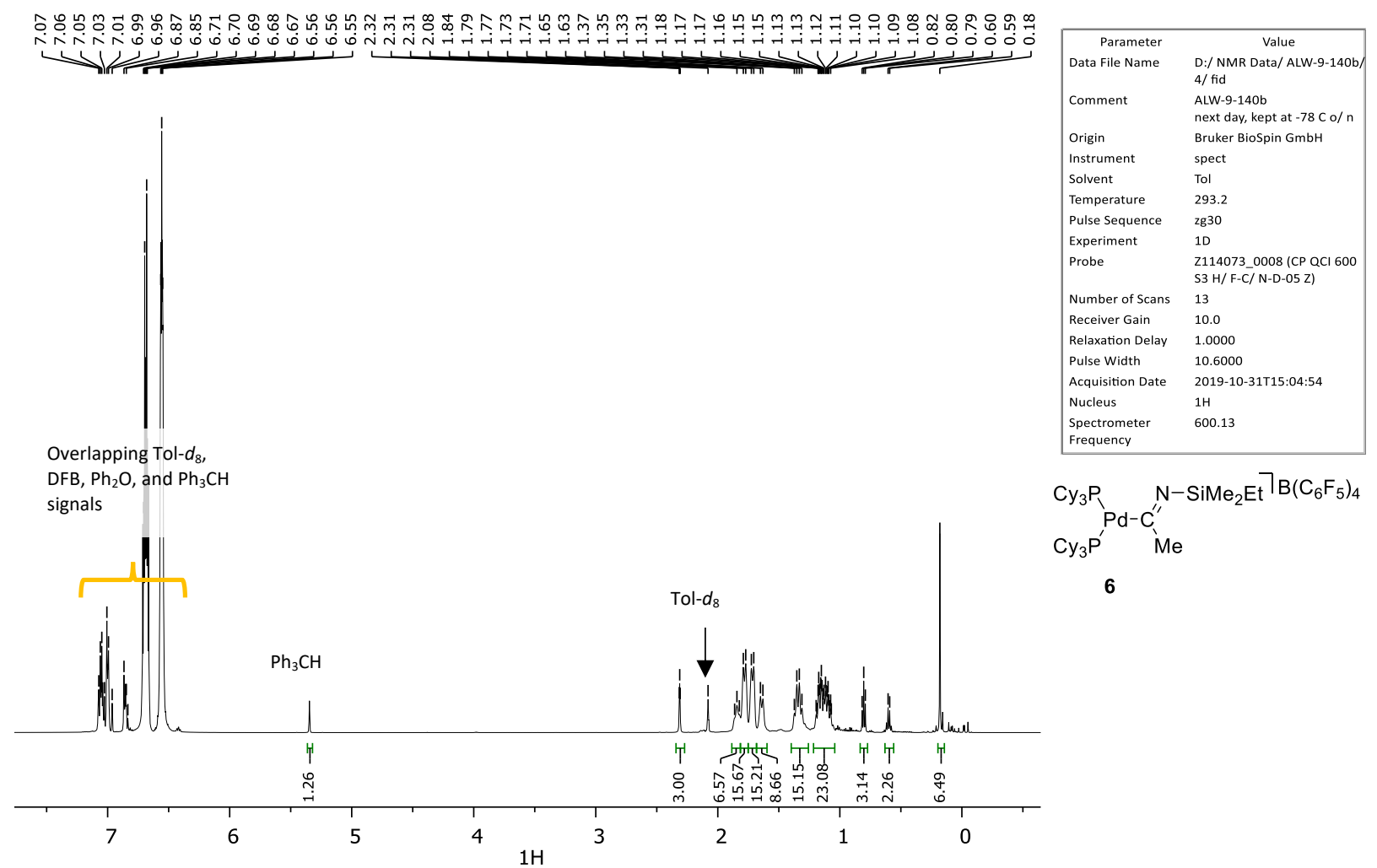

Figure S77: $600 \mathrm{MHz}{ }^{1} \mathrm{H}$ NMR spectrum of 6 at room temperature. Solvent: 1:5 DFB:Tol- $d_{8}$.

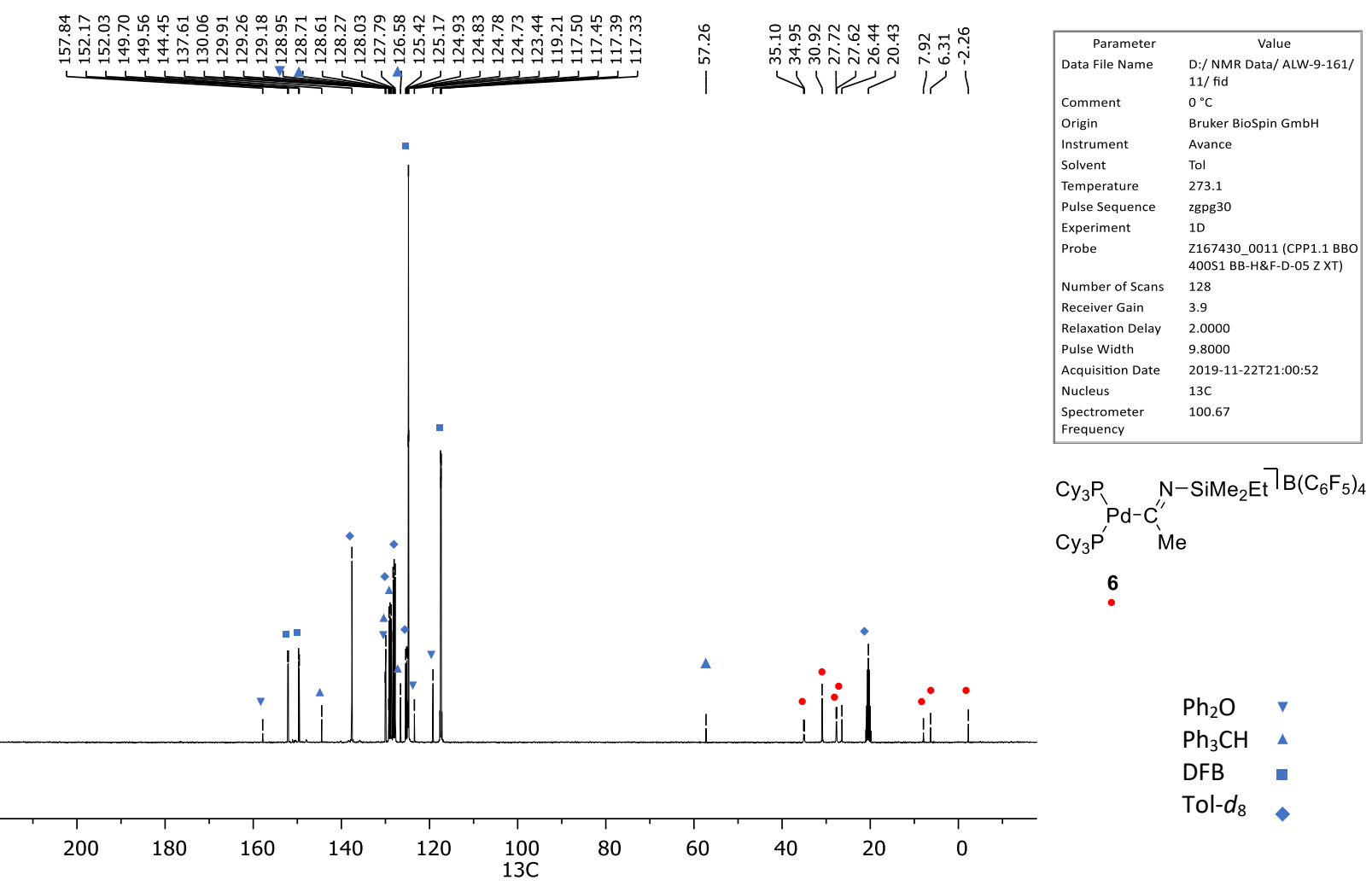

Figure S78: $101 \mathrm{MHz}{ }^{13} \mathrm{C}\left\{{ }^{1} \mathrm{H}\right\}$ NMR spectrum of 6 at room temperature. Solvent: 1:5 DFB:Tol- $d_{8}$. 


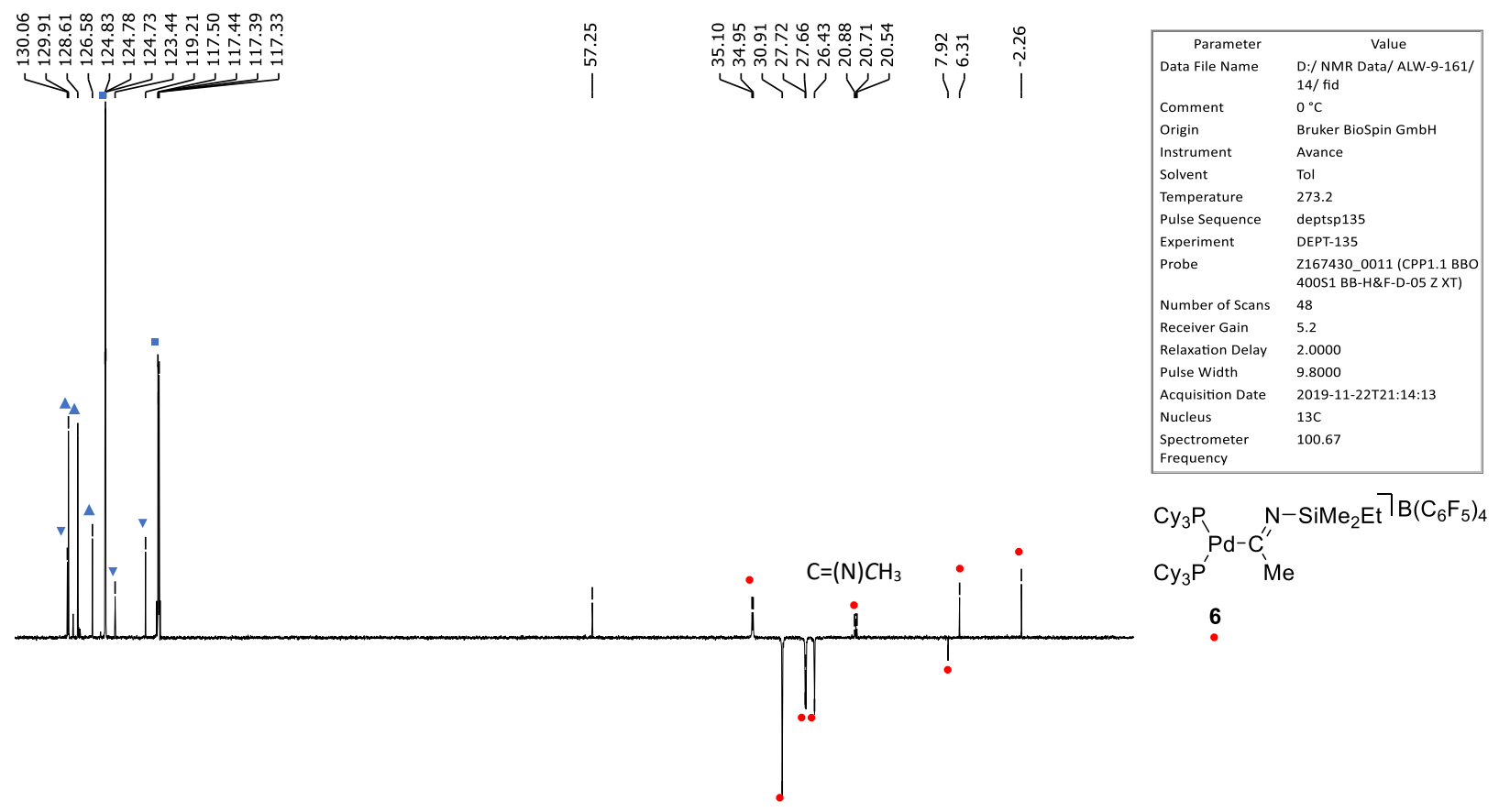

\begin{tabular}{|c|c|c|c|c|c|c|c|c|c|c|c|c|c|c|}
\hline 130 & 120 & 110 & 100 & 90 & 80 & 70 & $\begin{array}{c}60 \\
13 C\end{array}$ & 50 & 40 & 30 & 20 & 10 & 0 & -10 \\
\hline
\end{tabular}

Figure S79: $101 \mathrm{MHz}{ }^{13} \mathrm{C}\left\{{ }^{1} \mathrm{H}\right\}$ NMR spectrum of 6 at $0{ }^{\circ} \mathrm{C}$. Solvent: 1:5 DFB:Tol- $d_{8}$.

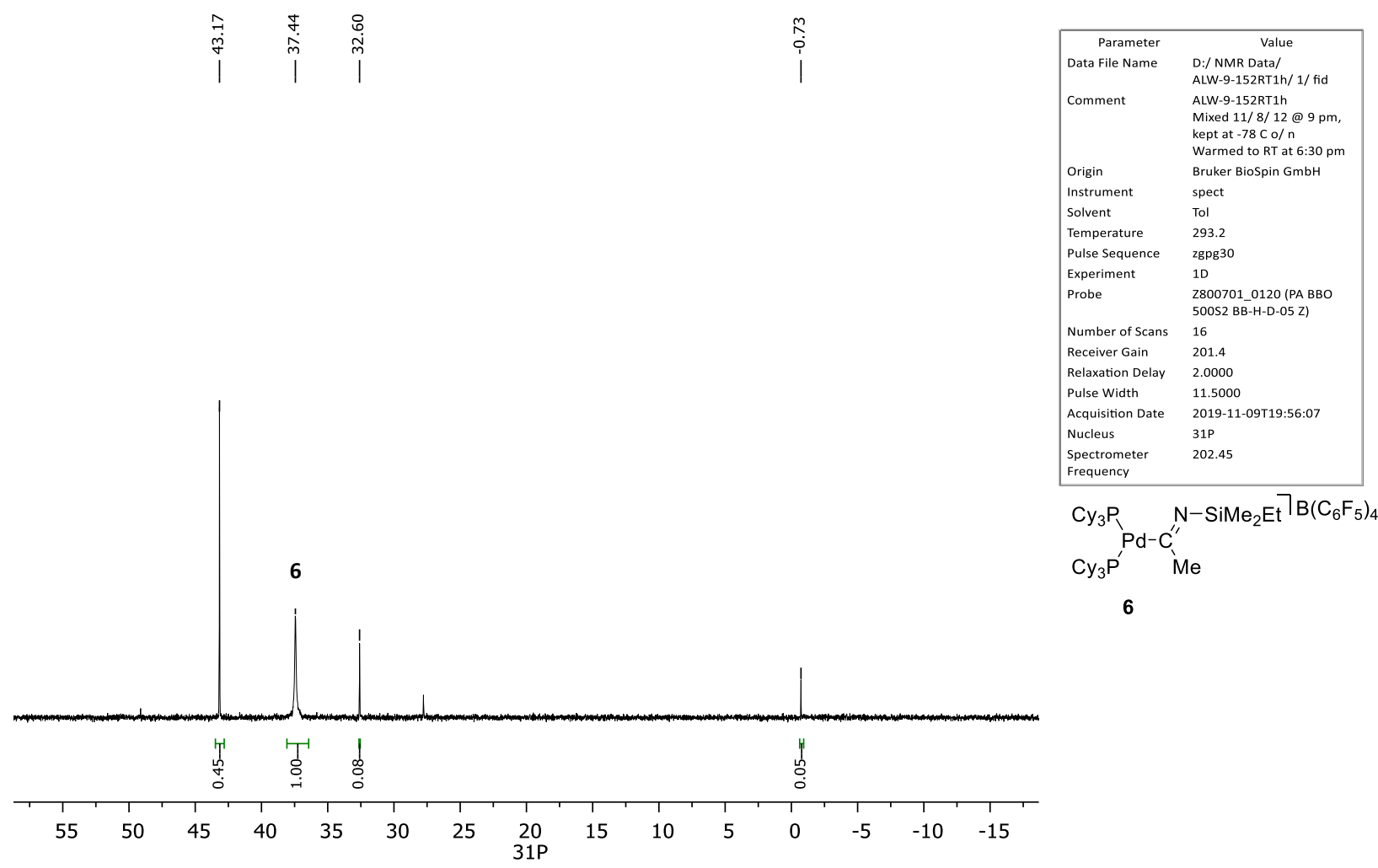

Figure S80: $202 \mathrm{MHz}{ }^{31} \mathrm{P}\left\{{ }^{1} \mathrm{H}\right\}$ NMR spectrum of 6 at room temperature. Solvent: 1:5 DFB:Tol- $d_{8}$. 


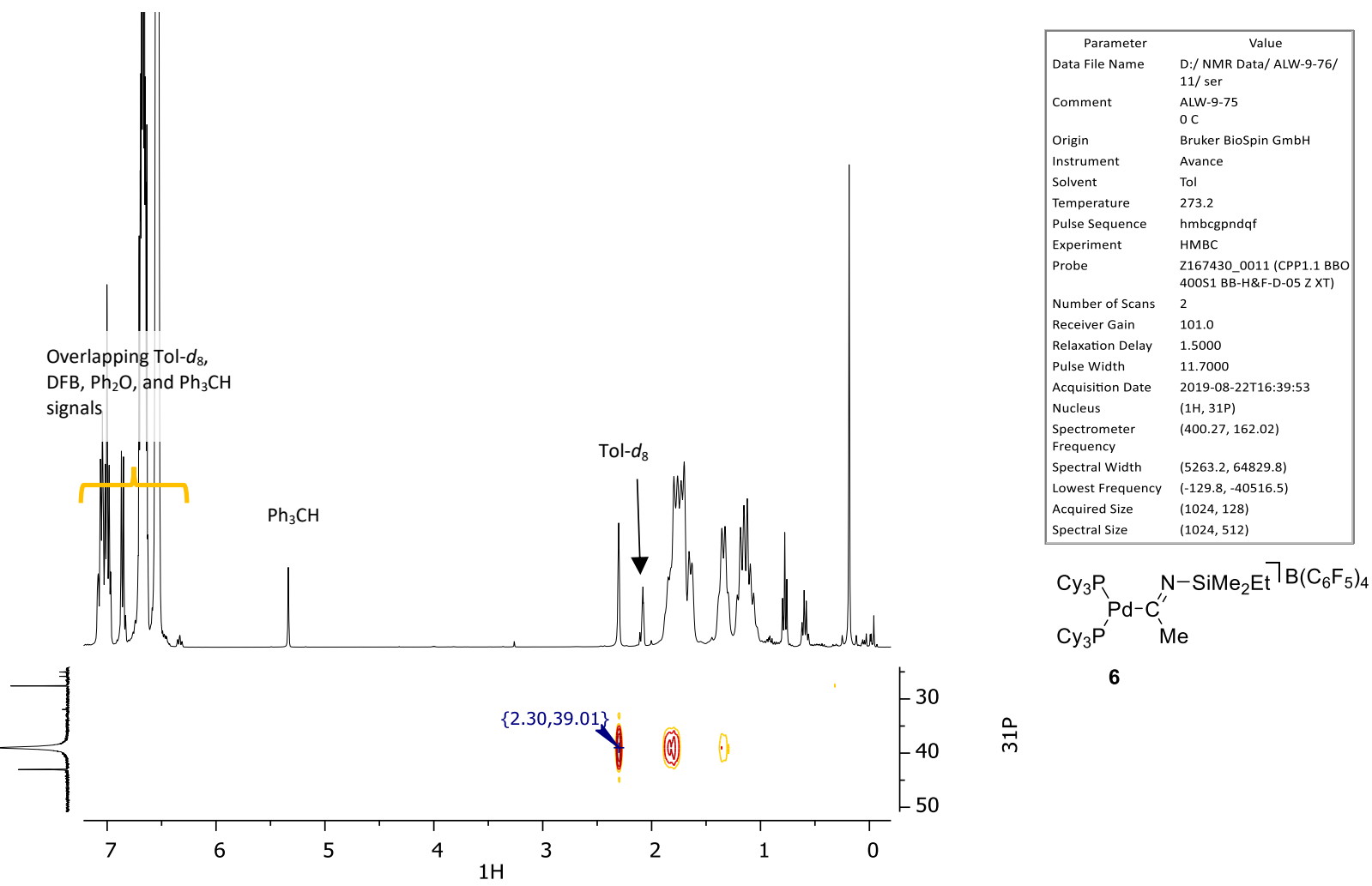

Figure S81: $(400 \mathrm{MHz}, 162 \mathrm{MHz}){ }^{1} \mathrm{H},{ }^{31} \mathrm{P}-\mathrm{HMBC}$ NMR spectrum of 6 at $0{ }^{\circ} \mathrm{C}$. Solvent: 1:5 DFB:Tol$d_{8}$.

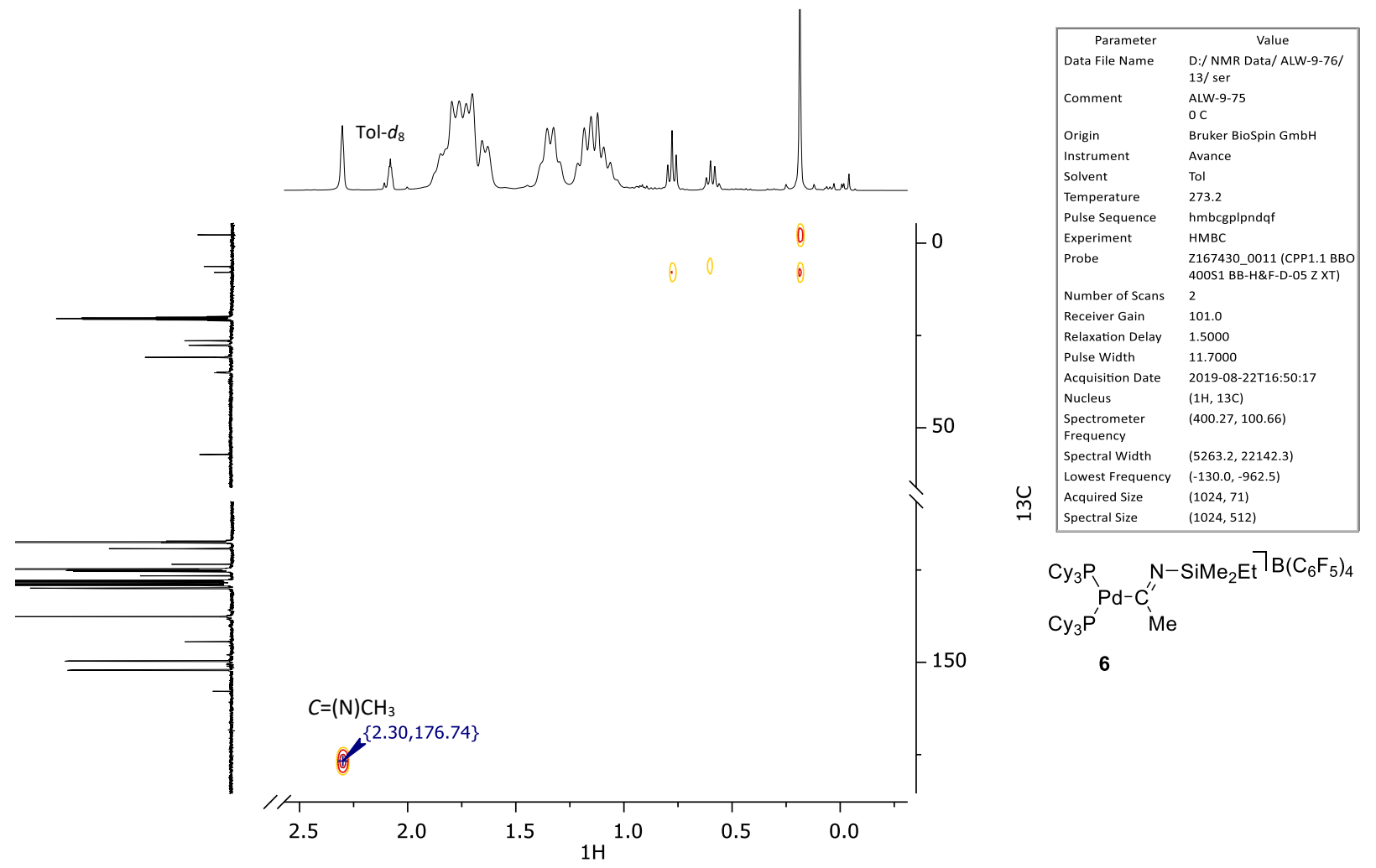

Figure S82: (400 MHz, $101 \mathrm{MHz}){ }^{1} \mathrm{H},{ }^{13} \mathrm{C}-\mathrm{HMBC}$ NMR spectrum of 6 at $0{ }^{\circ} \mathrm{C}$. Solvent: 1:5 DFB:Tol$d_{8}$. 


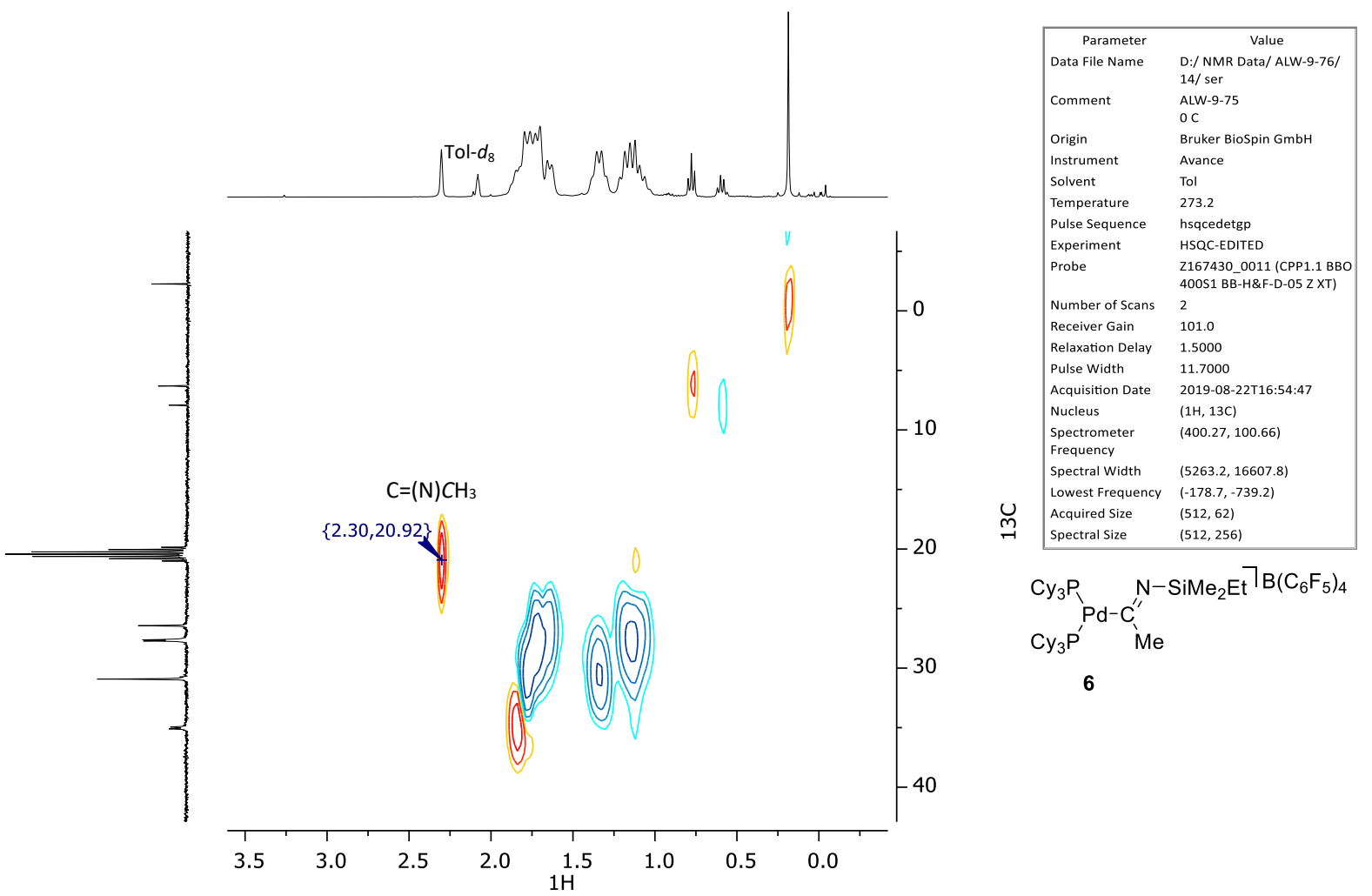

Figure S83: $(400 \mathrm{MHz}, 101 \mathrm{MHz}){ }^{1} \mathrm{H},{ }^{13} \mathrm{C}-\mathrm{HMBC}$ NMR spectrum of 6 at $0{ }^{\circ} \mathrm{C}$. Solvent: 1:5 DFB:Tol$d_{8}$.

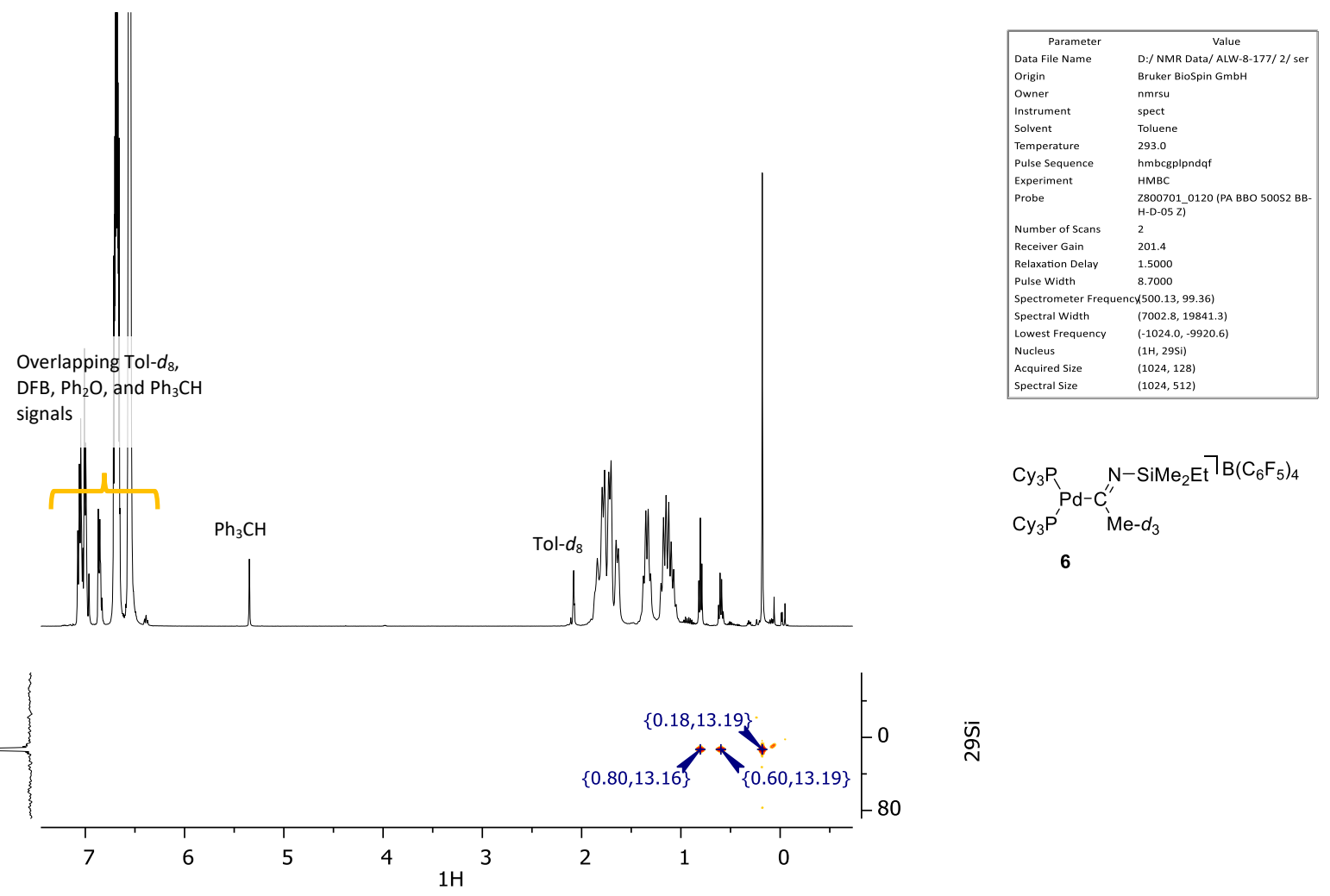

Figure S84: $(500 \mathrm{MHz}, 99 \mathrm{MHz}){ }^{1} \mathrm{H},{ }^{29} \mathrm{Si}-\mathrm{HMBC}$ NMR spectrum of 6 at room temperature. Solvent: 1:5 DFB:Tol-d8. 


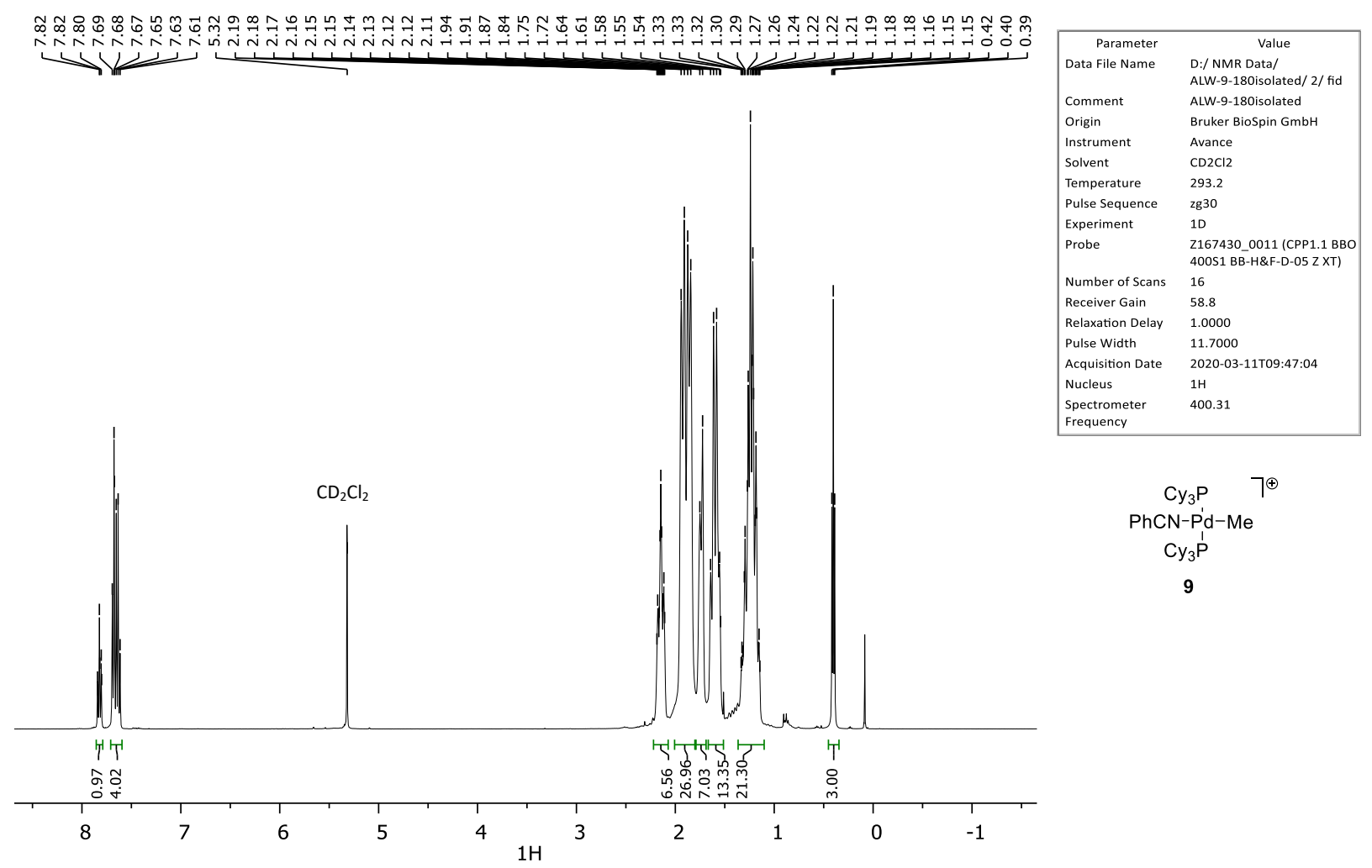

Figure S85: $400 \mathrm{MHz}{ }^{1} \mathrm{H}$ NMR spectrum of 9 at room temperature. Solvent: $\mathrm{CD}_{2} \mathrm{Cl}_{2}$.

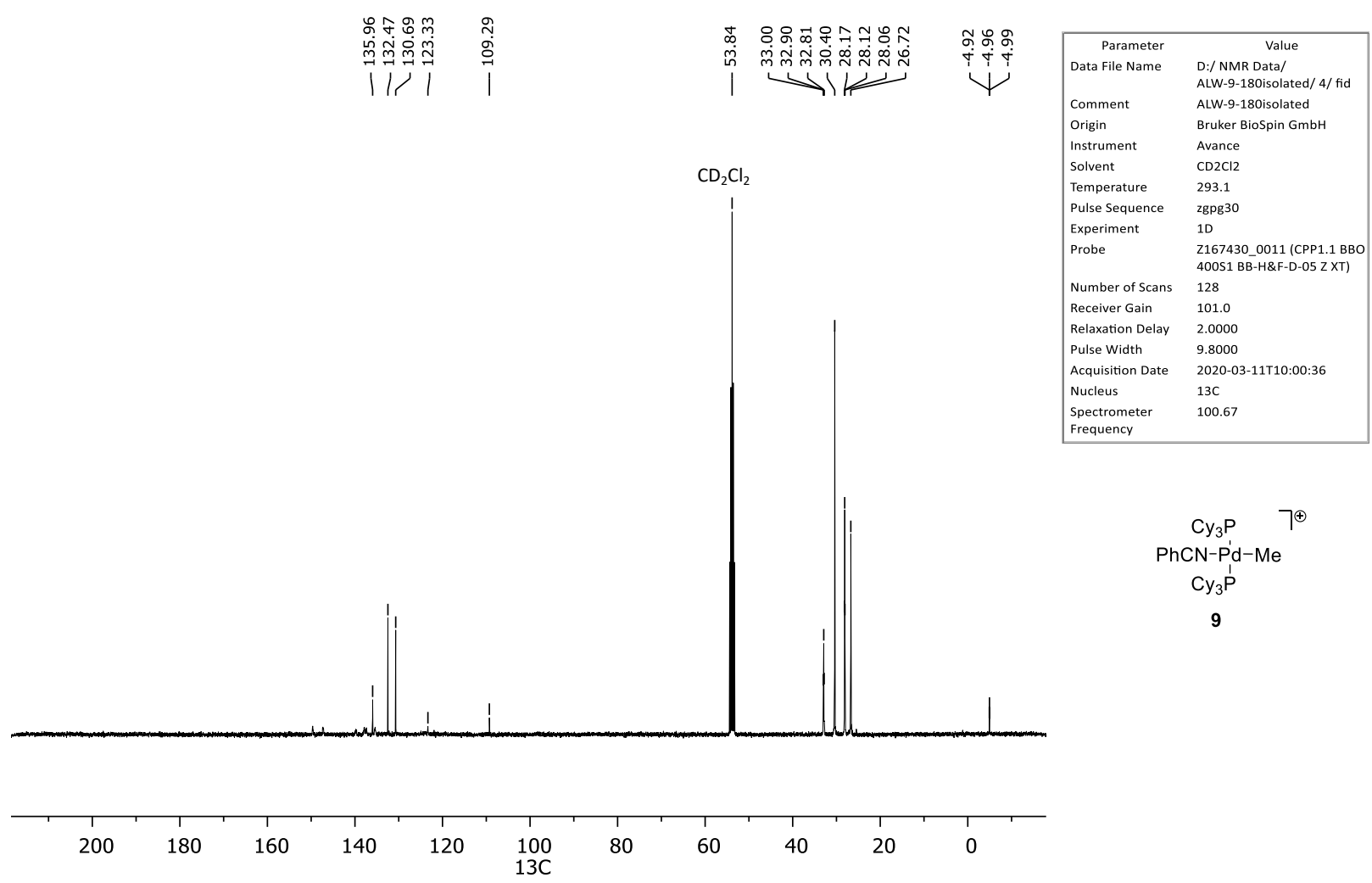

Figure S86: $101 \mathrm{MHz}{ }^{13} \mathrm{C}\left\{{ }^{1} \mathrm{H}\right\}$ NMR spectrum of 9 at room temperature. Solvent: $\mathrm{CD}_{2} \mathrm{Cl}_{2}$. 


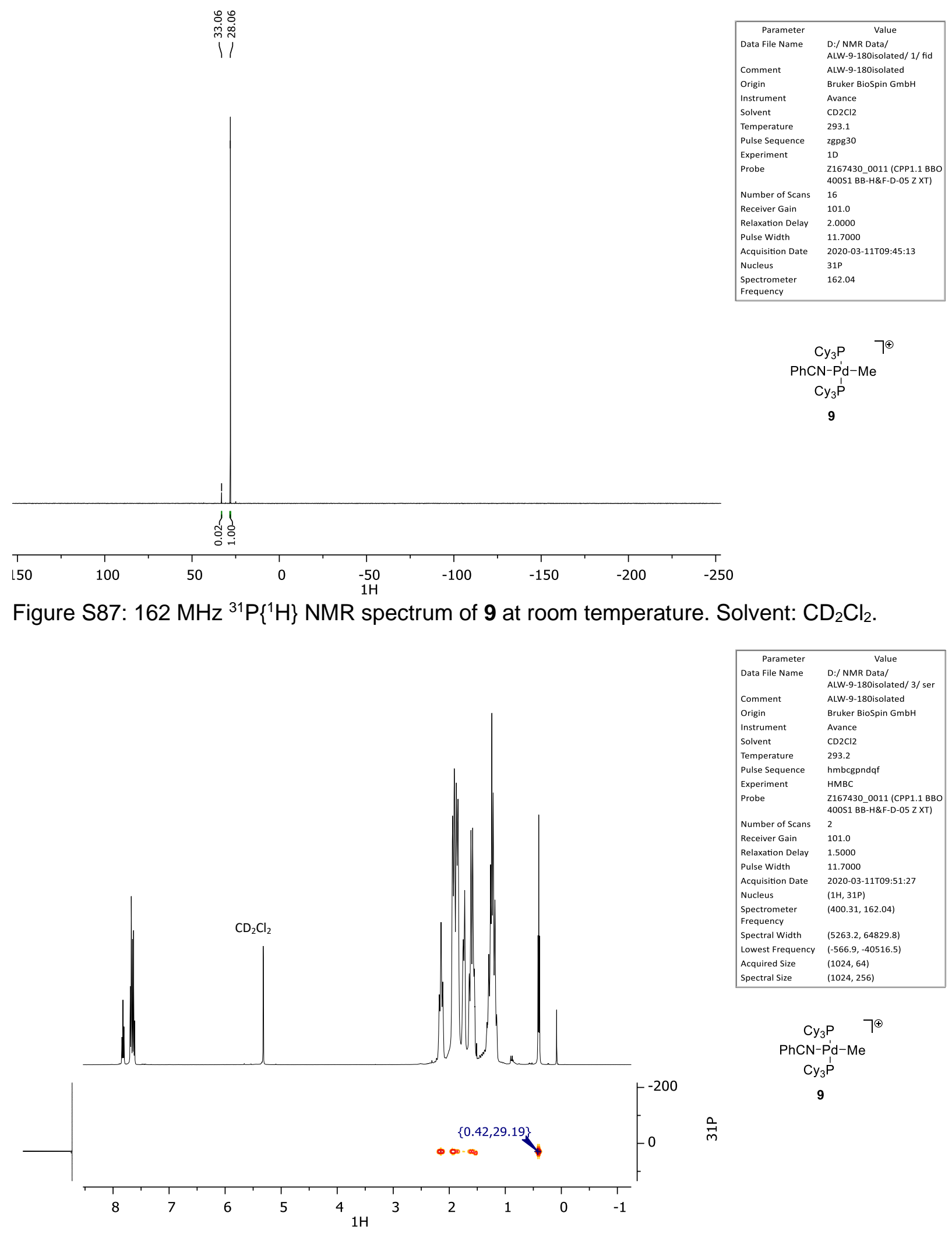

Figure S88: (400 MHz, 162) ${ }^{1} \mathrm{H},{ }^{31} \mathrm{P}-\mathrm{HMBC}$ NMR spectrum of 9 at room temperature. Solvent: $\mathrm{CD}_{2} \mathrm{Cl}_{2}$. 


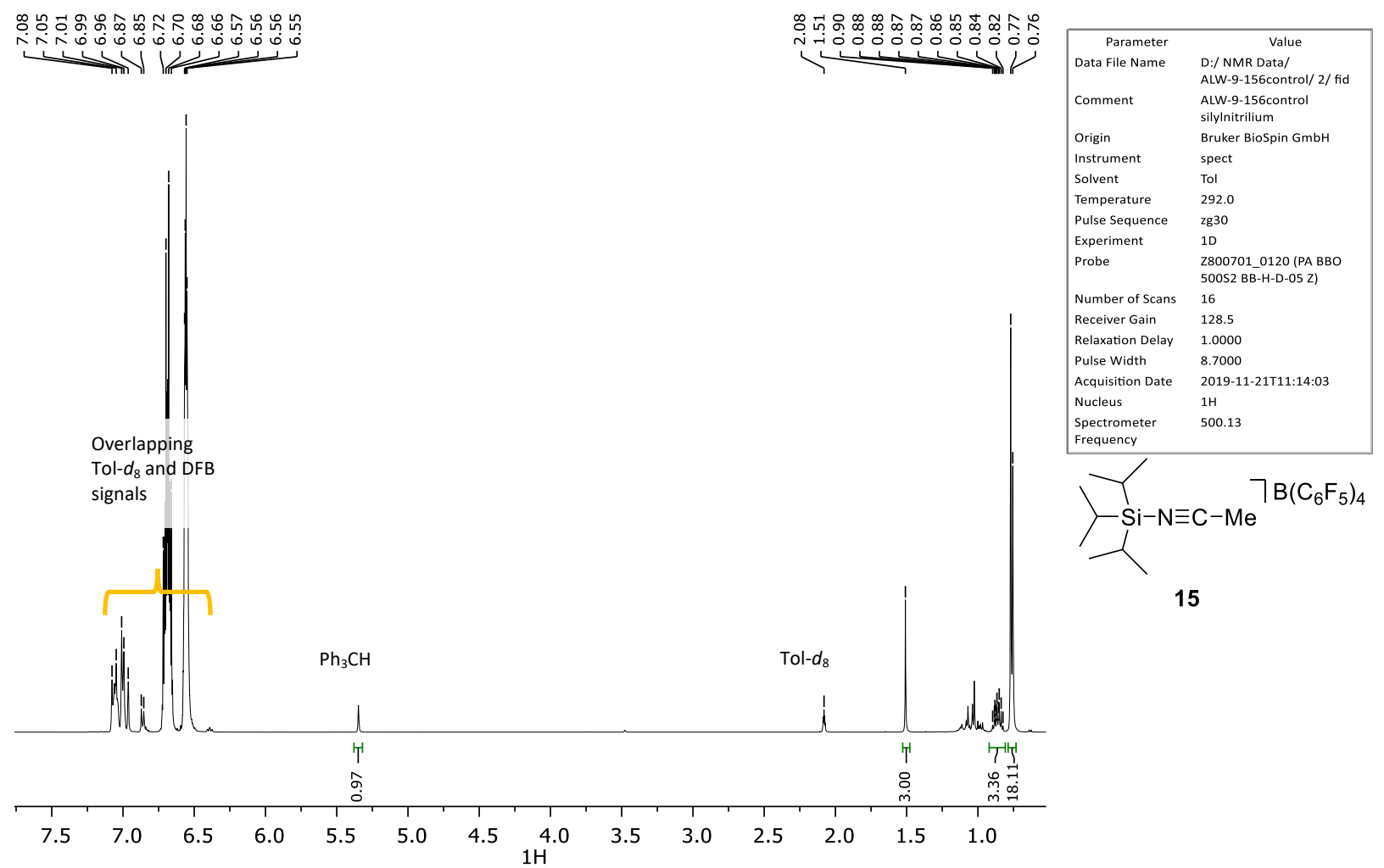

Figure S89: $500 \mathrm{MHz}{ }^{1} \mathrm{H}$ NMR spectrum of 15 at room temperature. Solvent: 1:5 DFB:Tol- $d_{8}$.
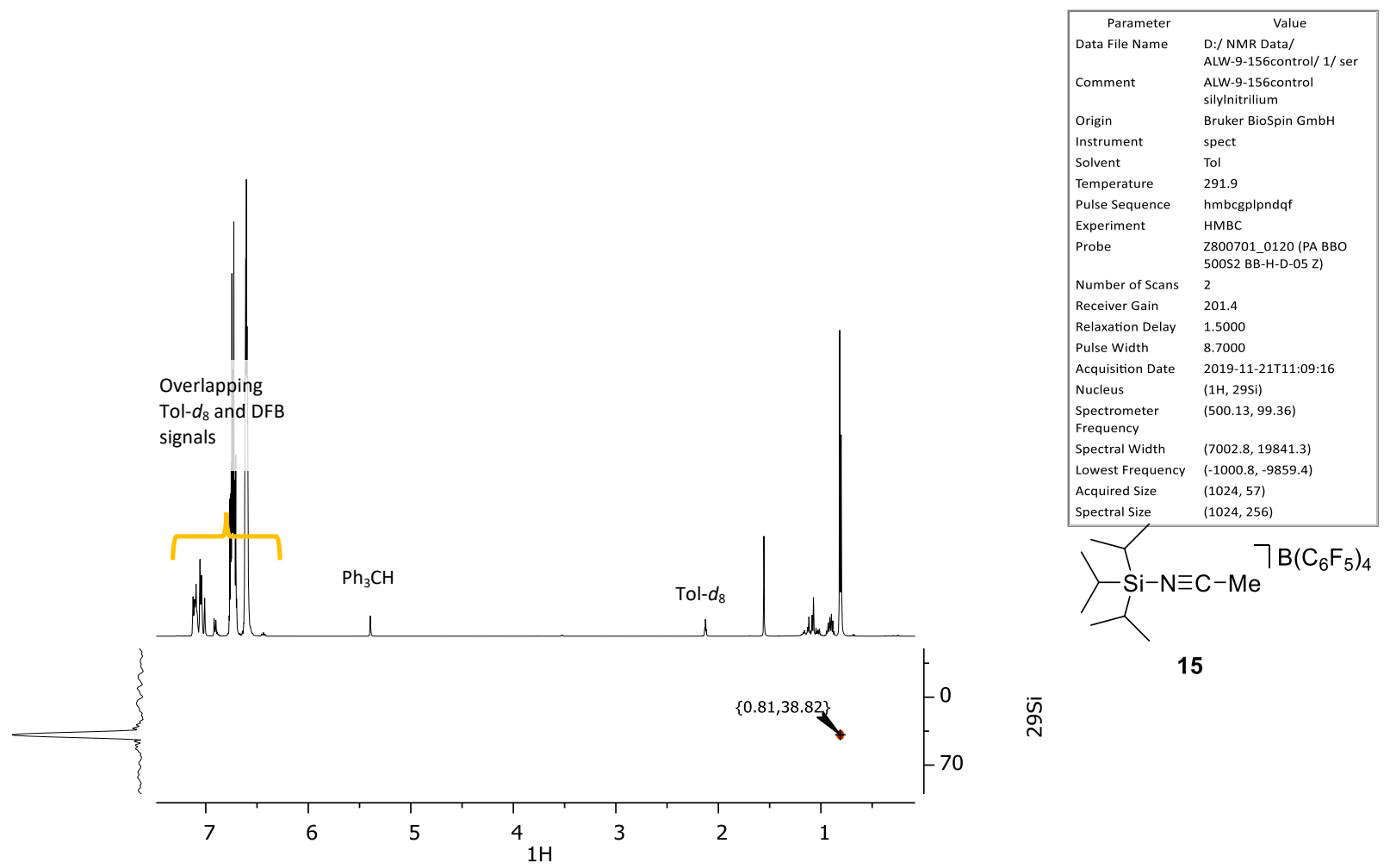

15

Figure S90: $(500 \mathrm{MHz}, 99 \mathrm{MHz}){ }^{1} \mathrm{H},{ }^{29} \mathrm{Si}-\mathrm{HMBC}$ NMR spectrum of 15 at room temperature. Solvent: 1:5 DFB:Tol- $d_{8}$. 


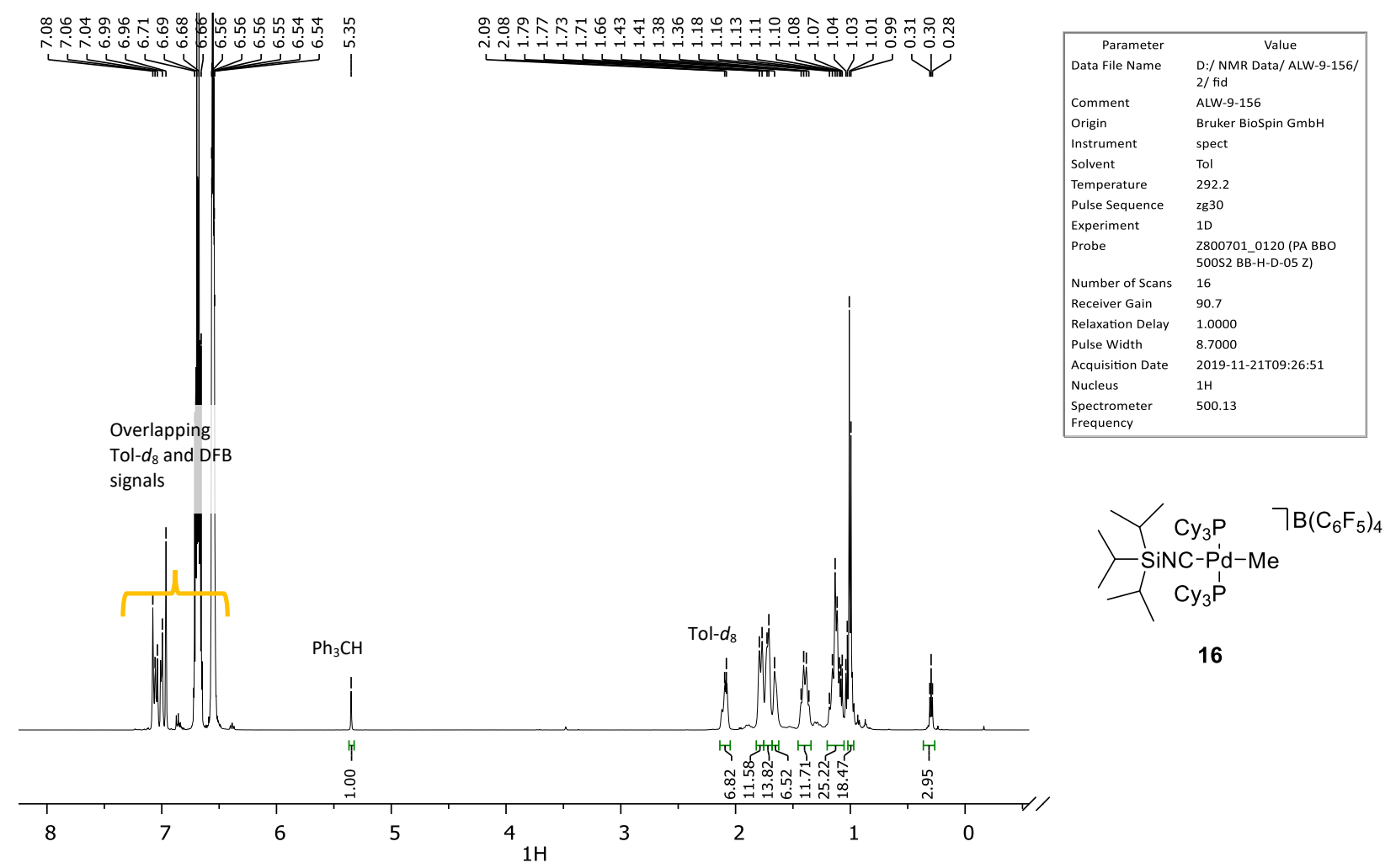

Figure S91: $500 \mathrm{MHz}{ }^{1} \mathrm{H}$ NMR spectrum of 16 at room temperature. Solvent: 1:5 DFB:Tol- $d_{8}$.

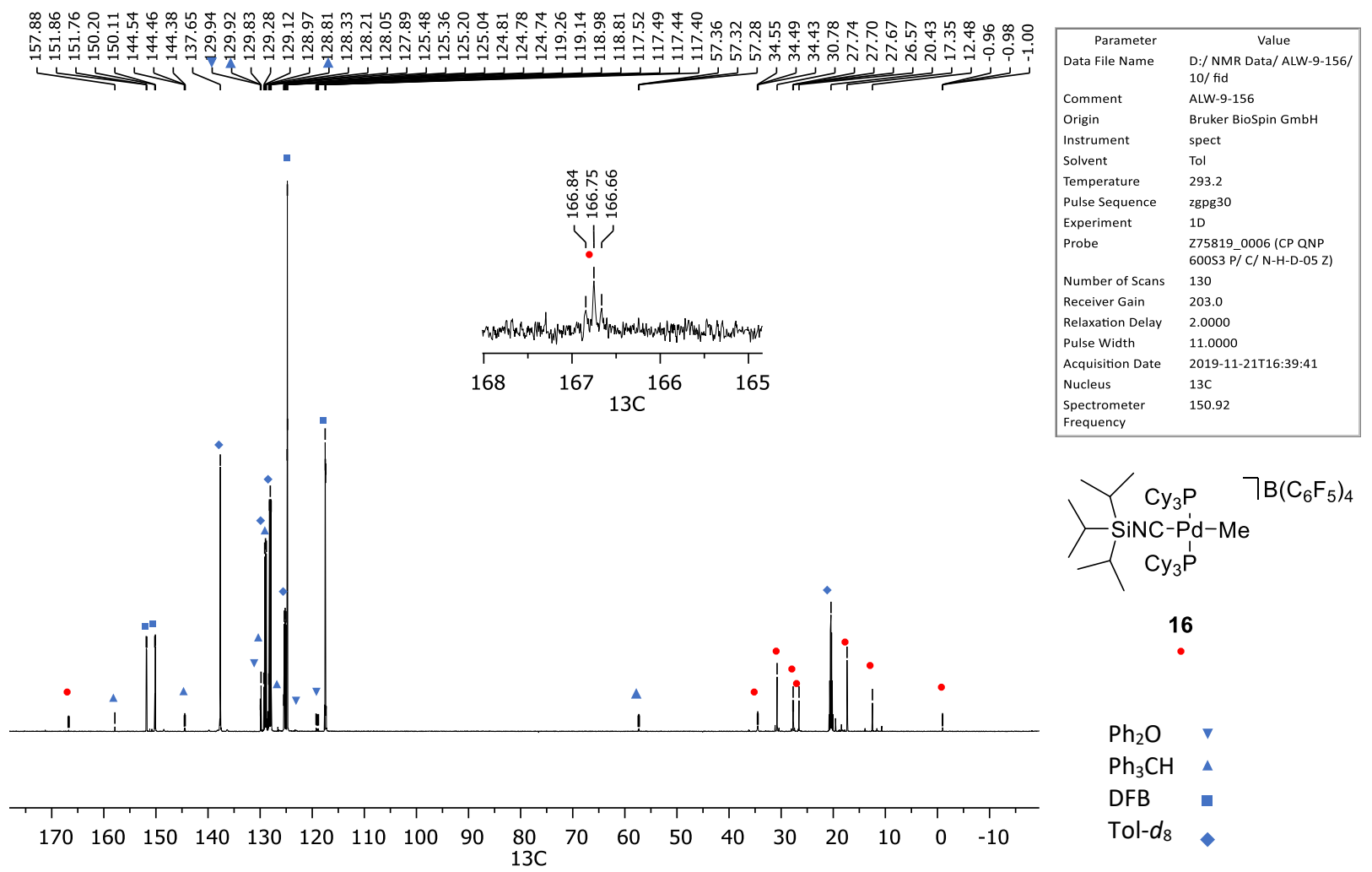

Figure S92: $151 \mathrm{MHz}{ }^{13} \mathrm{C}\left\{{ }^{1} \mathrm{H}\right\}$ NMR spectrum of 16 at room temperature. Solvent: 1:5 DFB:Tol$d_{8}$. 


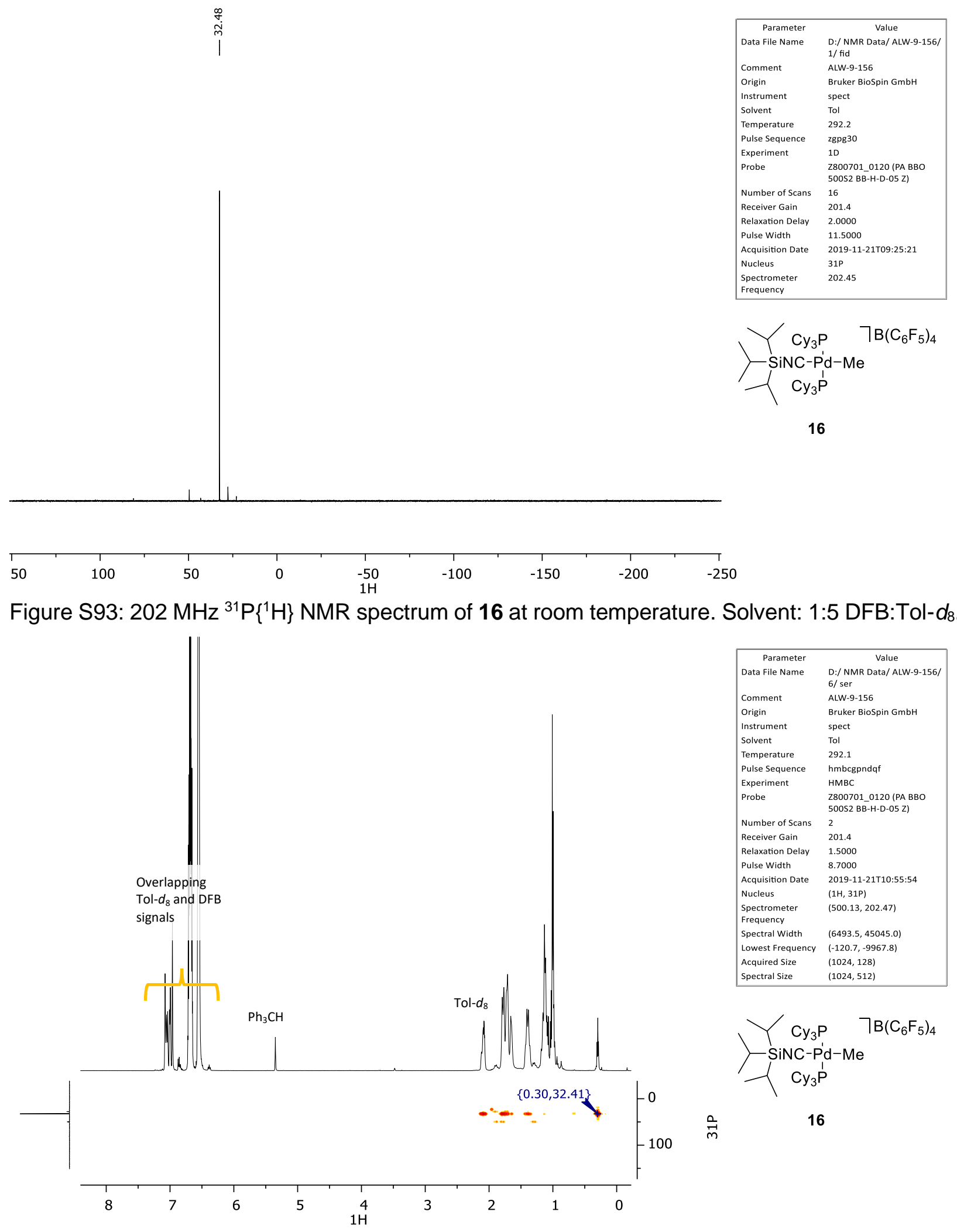

Figure S94: (500 MHz, $202 \mathrm{MHz}){ }^{1} \mathrm{H},{ }^{31} \mathrm{P}-\mathrm{HMBC}$ NMR spectrum of 16 at room temperature. Solvent: 1:5 DFB:Tol- $d_{8}$. 


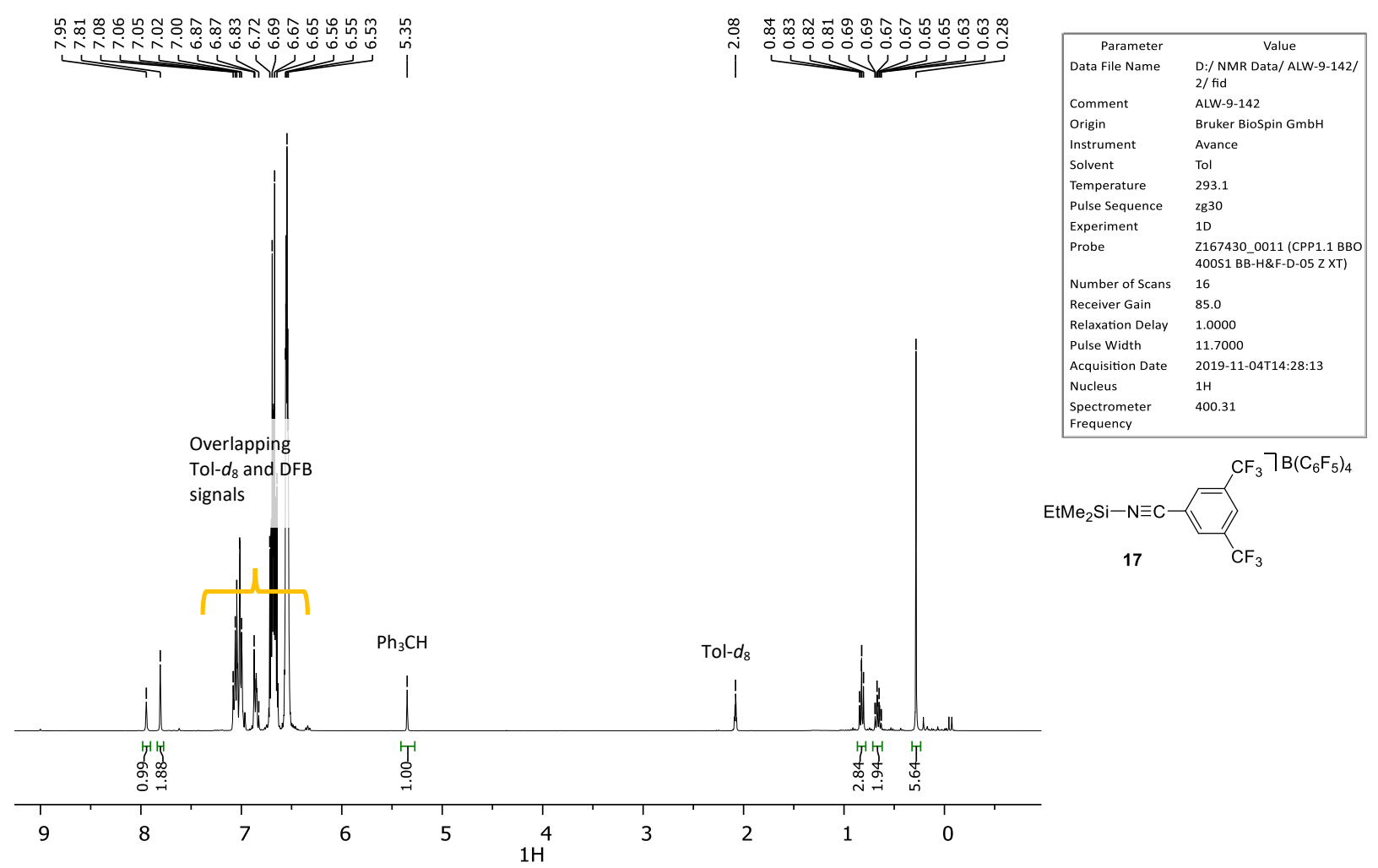

Figure S95: $400 \mathrm{MHz}{ }^{1} \mathrm{H}$ NMR spectrum of 17 at room temperature. Solvent: 1:5 DFB:Tol- $d_{8}$.

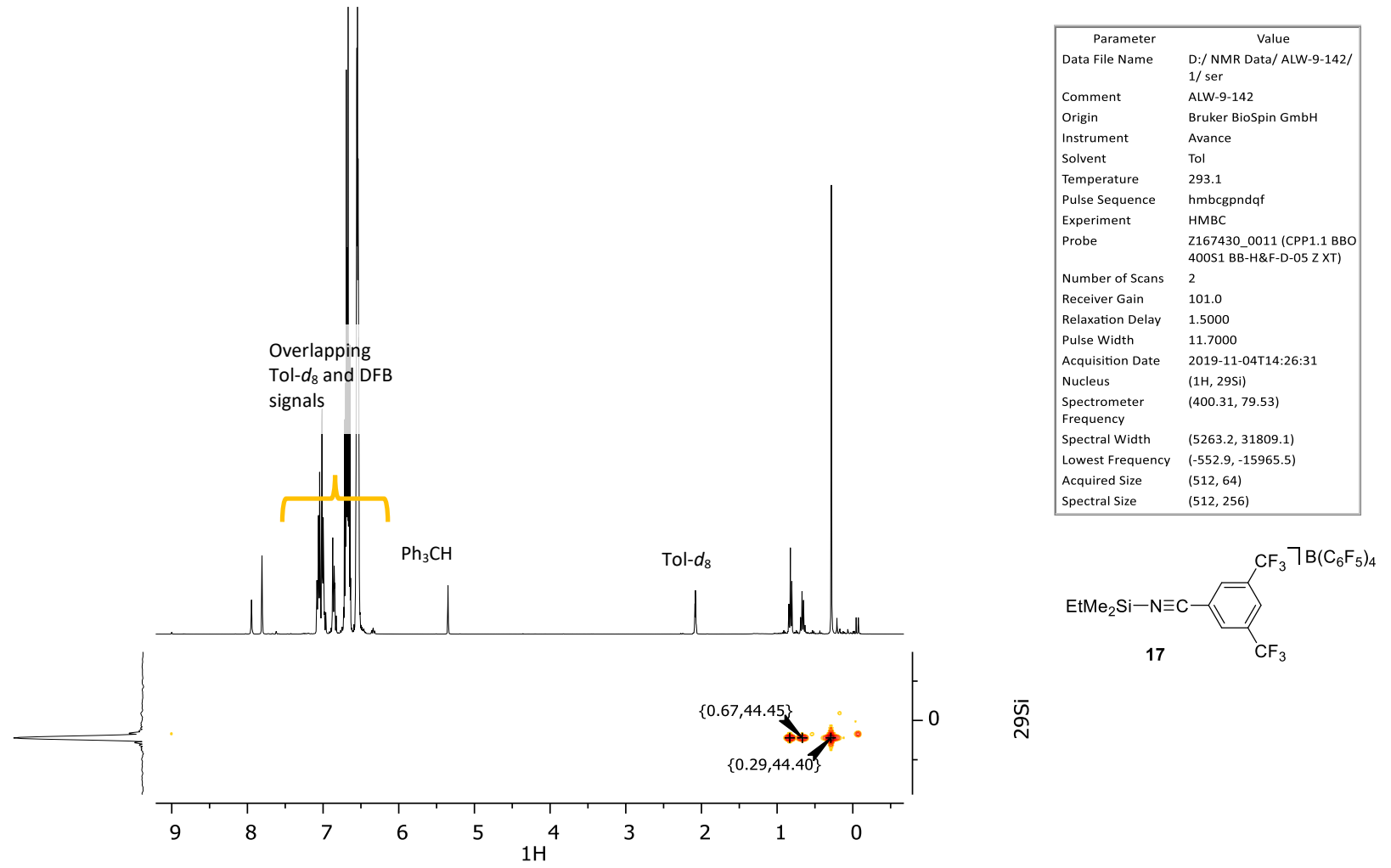

Figure S96: (400 MHz, $80 \mathrm{MHz}){ }^{1} \mathrm{H}^{29}{ }^{29} \mathrm{Si}-\mathrm{HMBC}$ NMR spectrum of 17 at room temperature. Solvent: 1:5 DFB:Tol- $d_{8}$. 


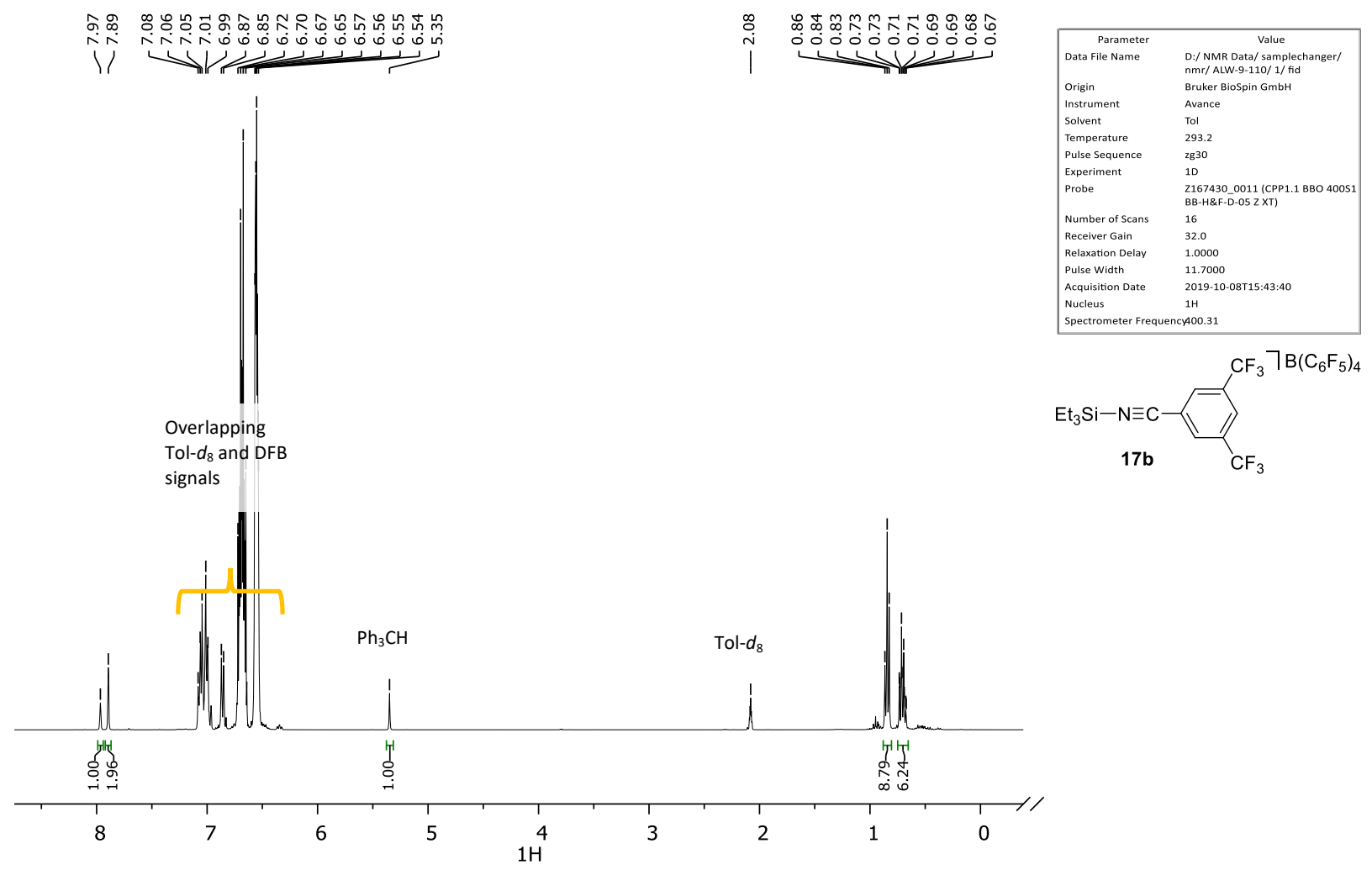

Figure S97: $400 \mathrm{MHz}{ }^{1} \mathrm{H}$ NMR spectrum of $17 \mathrm{~b}$ at room temperature. Solvent: 1:5 DFB:Tol- $d_{8}$.

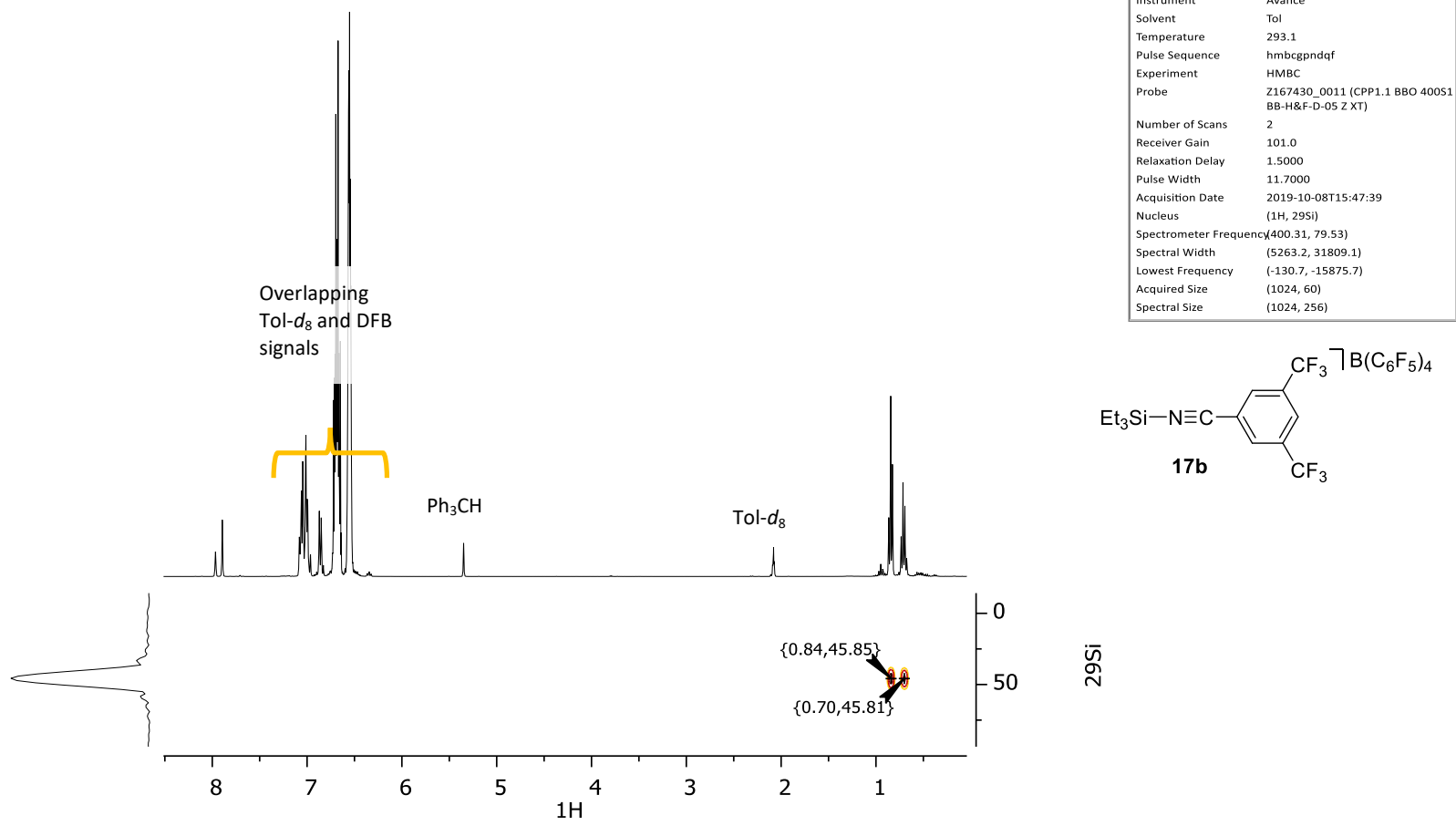

Figure S98: (400 MHz, $80 \mathrm{MHz}){ }^{1} \mathrm{H},{ }^{29} \mathrm{Si}-\mathrm{HMBC}$ NMR spectrum of 17 at room temperature. Solvent: 1:5 DFB:Tol- $d_{8}$. 


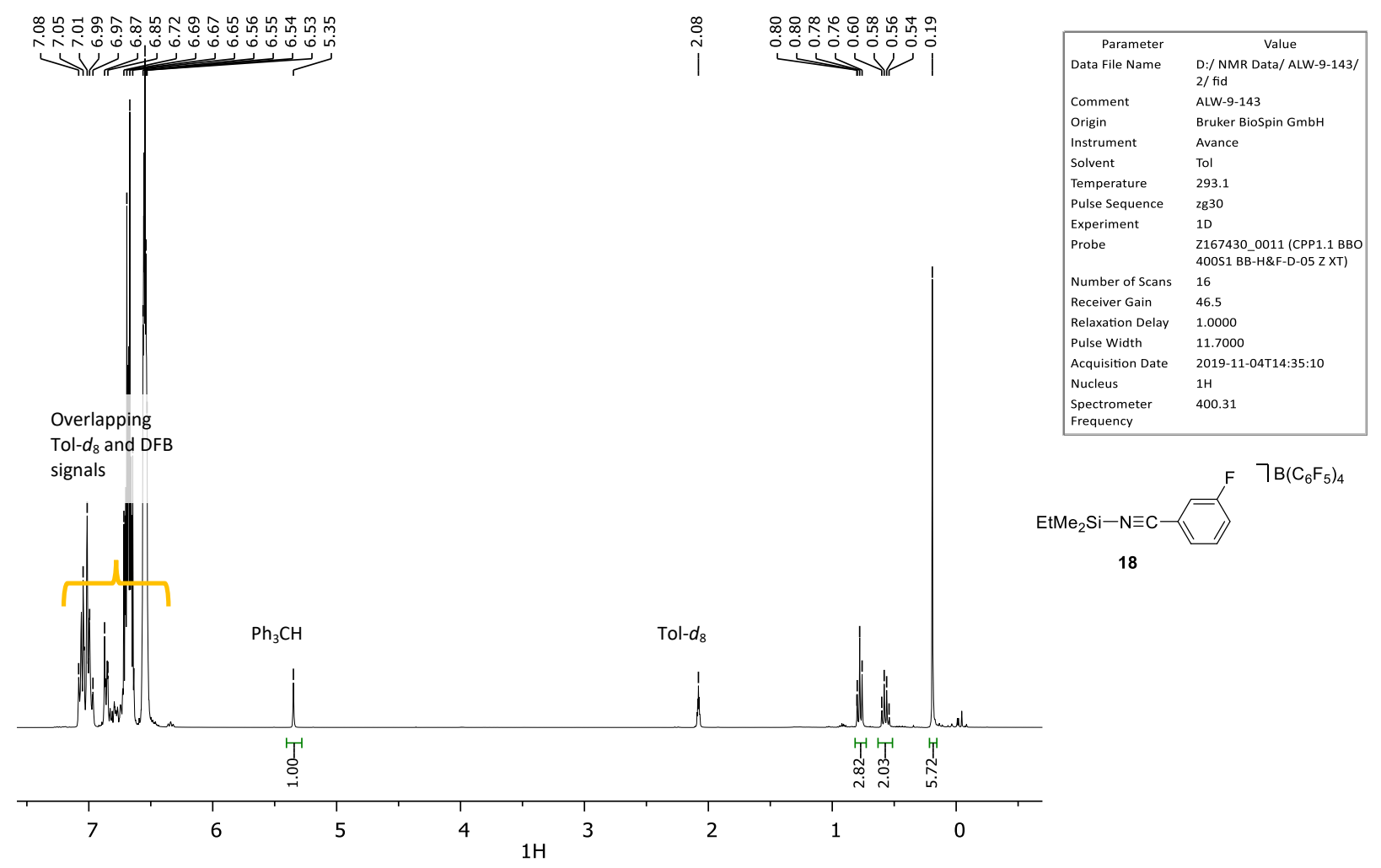

Figure S99: $400 \mathrm{MHz}{ }^{1} \mathrm{H}$ NMR spectrum of 18 at room temperature. Solvent: 1:5 DFB:Tol- $d_{8}$.

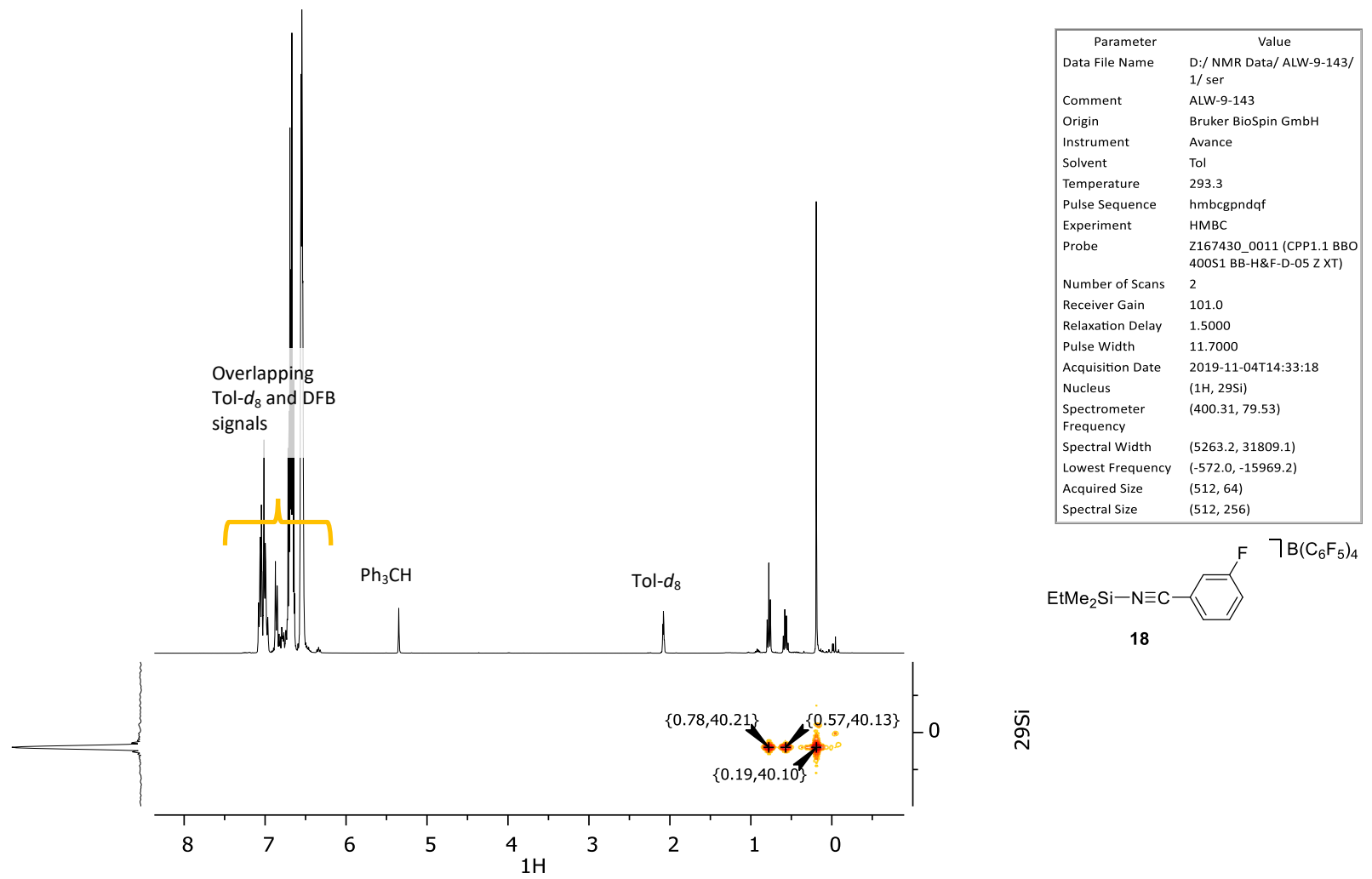

Figure S100: (400 MHz, $80 \mathrm{MHz}){ }^{1} \mathrm{H},{ }^{29} \mathrm{Si}-\mathrm{HMBC}$ NMR spectrum of 18 at room temperature. Solvent: 1:5 DFB:Tol- $d_{8}$. 


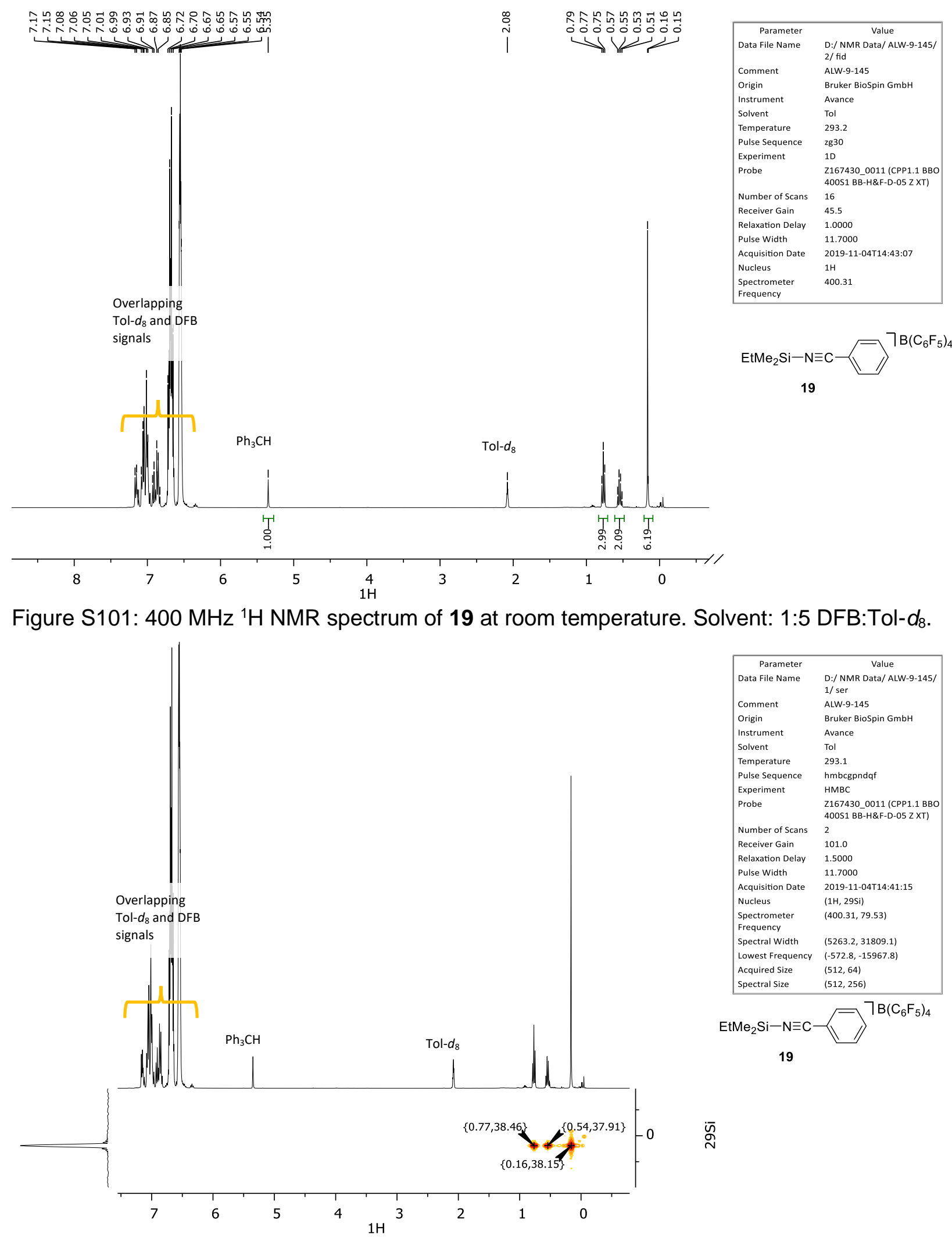

Figure S102: (400 MHz, $80 \mathrm{MHz}$ ) ${ }^{1} \mathrm{H},{ }^{29} \mathrm{Si}-\mathrm{HMBC}$ NMR spectrum of 19 at room temperature. Solvent: 1:5 DFB:Tol- $d_{8}$. 


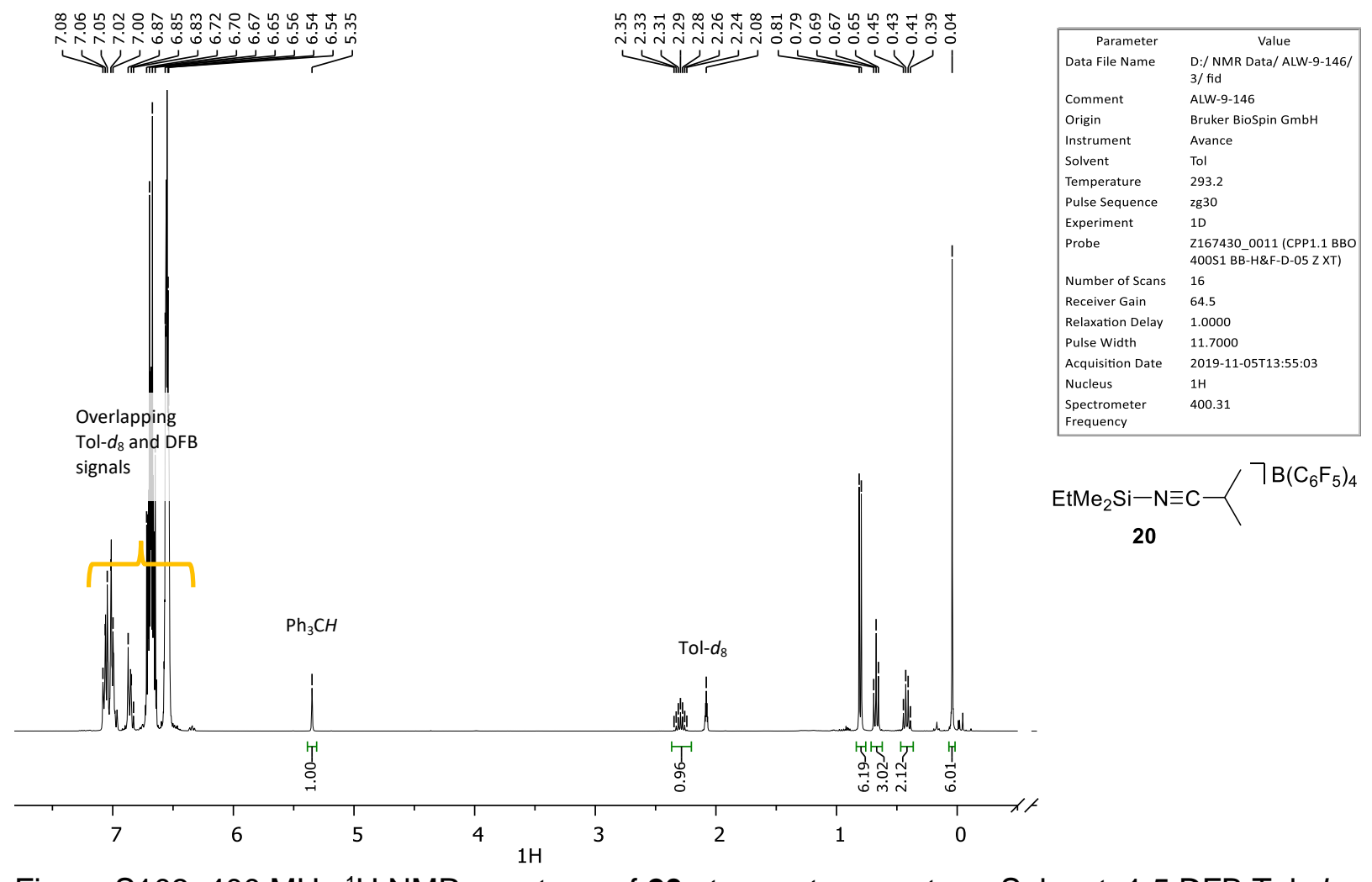

Figure S103: $400 \mathrm{MHz}{ }^{1} \mathrm{H}$ NMR spectrum of 20 at room temperature. Solvent: 1:5 DFB:Tol- $d_{8}$.

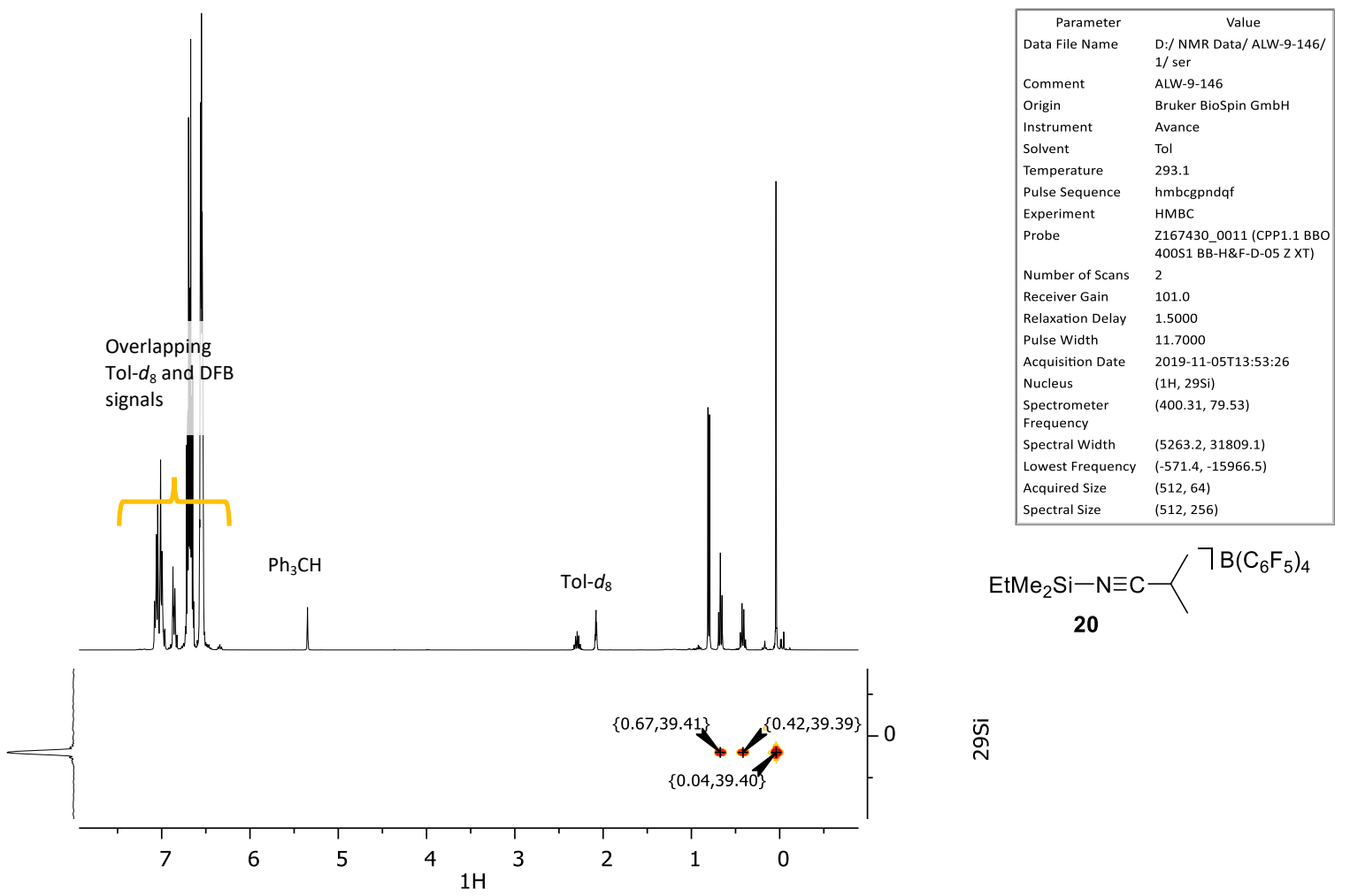

Figure S104: (400 MHz, $80 \mathrm{MHz}){ }^{1} \mathrm{H},{ }^{29} \mathrm{Si}-\mathrm{HMBC}$ NMR spectrum of 20 at room temperature. Solvent: 1:5 DFB:Tol- $d_{8}$. 

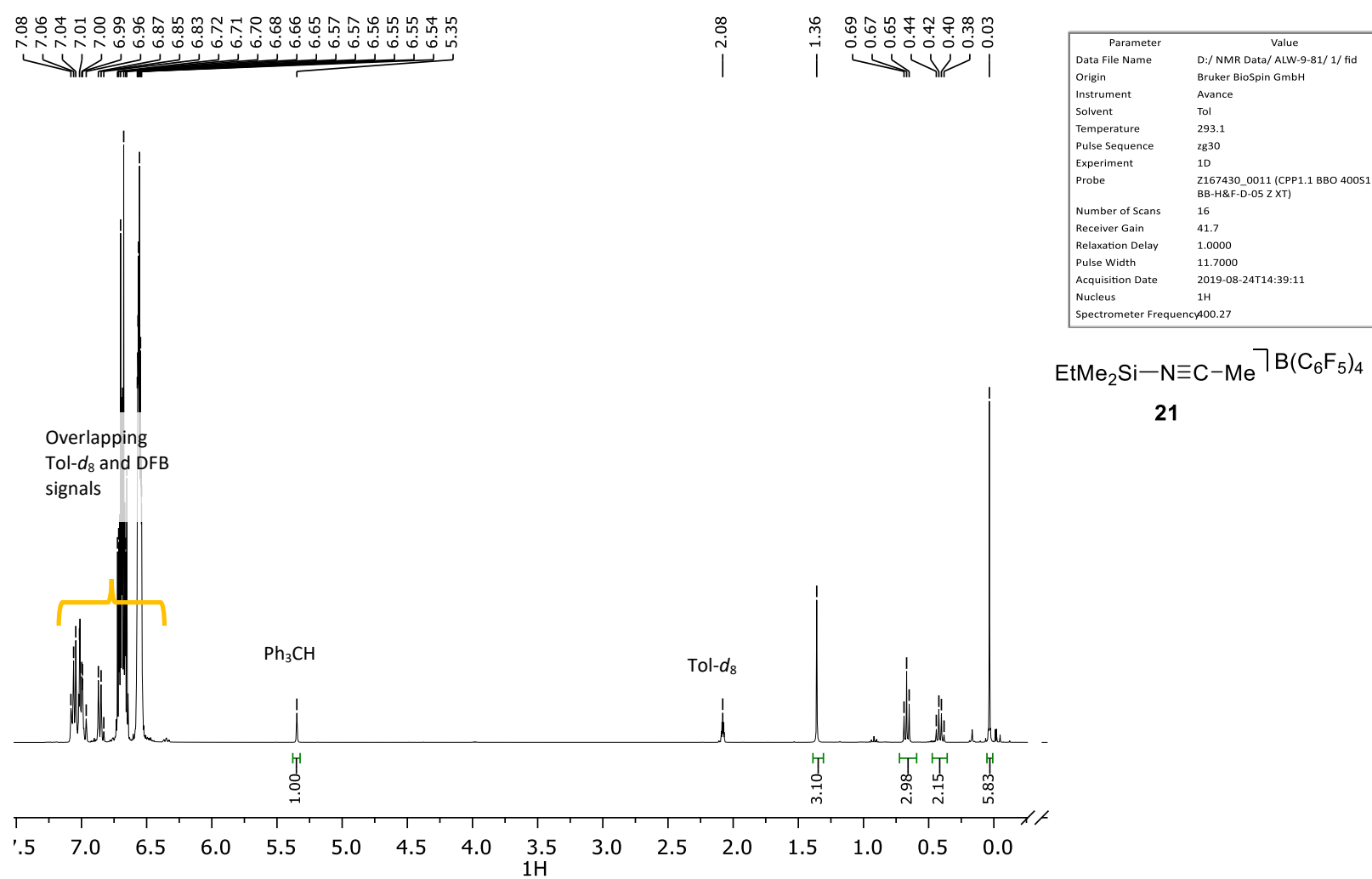

$\mathrm{EtMe}_{2} \mathrm{Si}-\mathrm{N} \equiv \mathrm{C}-\mathrm{Me} \backslash \mathrm{B}\left(\mathrm{C}_{6} \mathrm{~F}_{5}\right)_{4}$

21

Figure S105: $400 \mathrm{MHz}{ }^{1} \mathrm{H}$ NMR spectrum of 21 at room temperature. Solvent: 1:5 DFB:Tol- $d_{8}$.

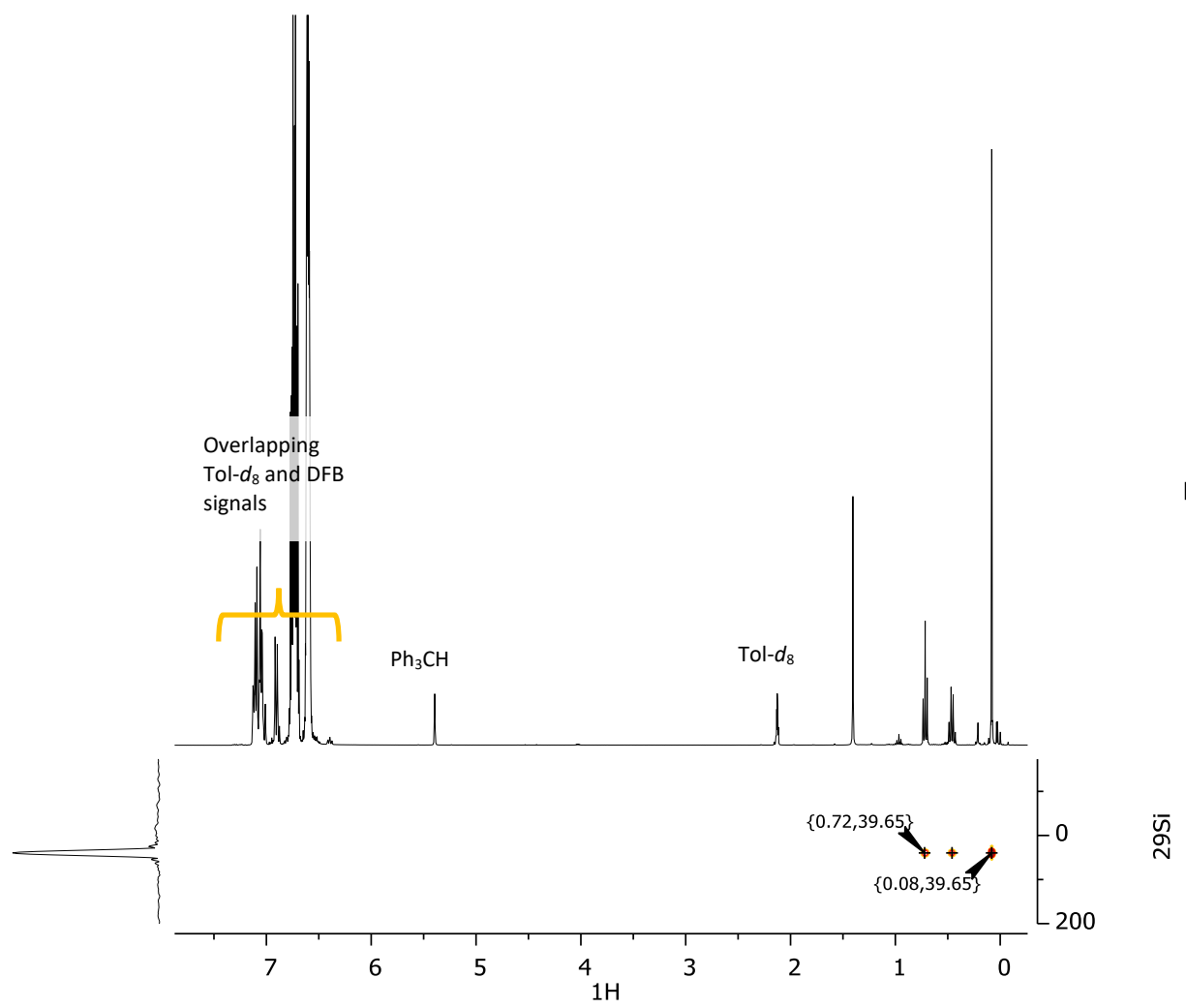

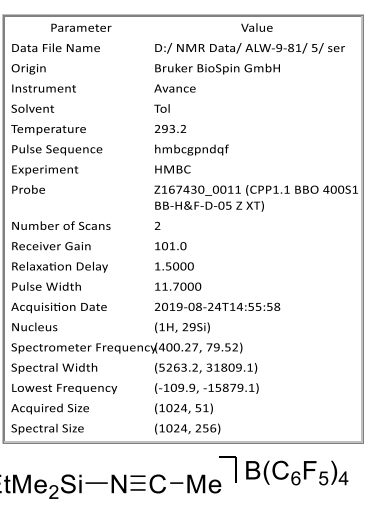

21

Figure S106: (400 MHz, $80 \mathrm{MHz}){ }^{1} \mathrm{H},{ }^{29} \mathrm{Si}-\mathrm{HMBC}$ NMR spectrum of 21 at room temperature. Solvent: 1:5 DFB:Tol- $d_{8}$. 

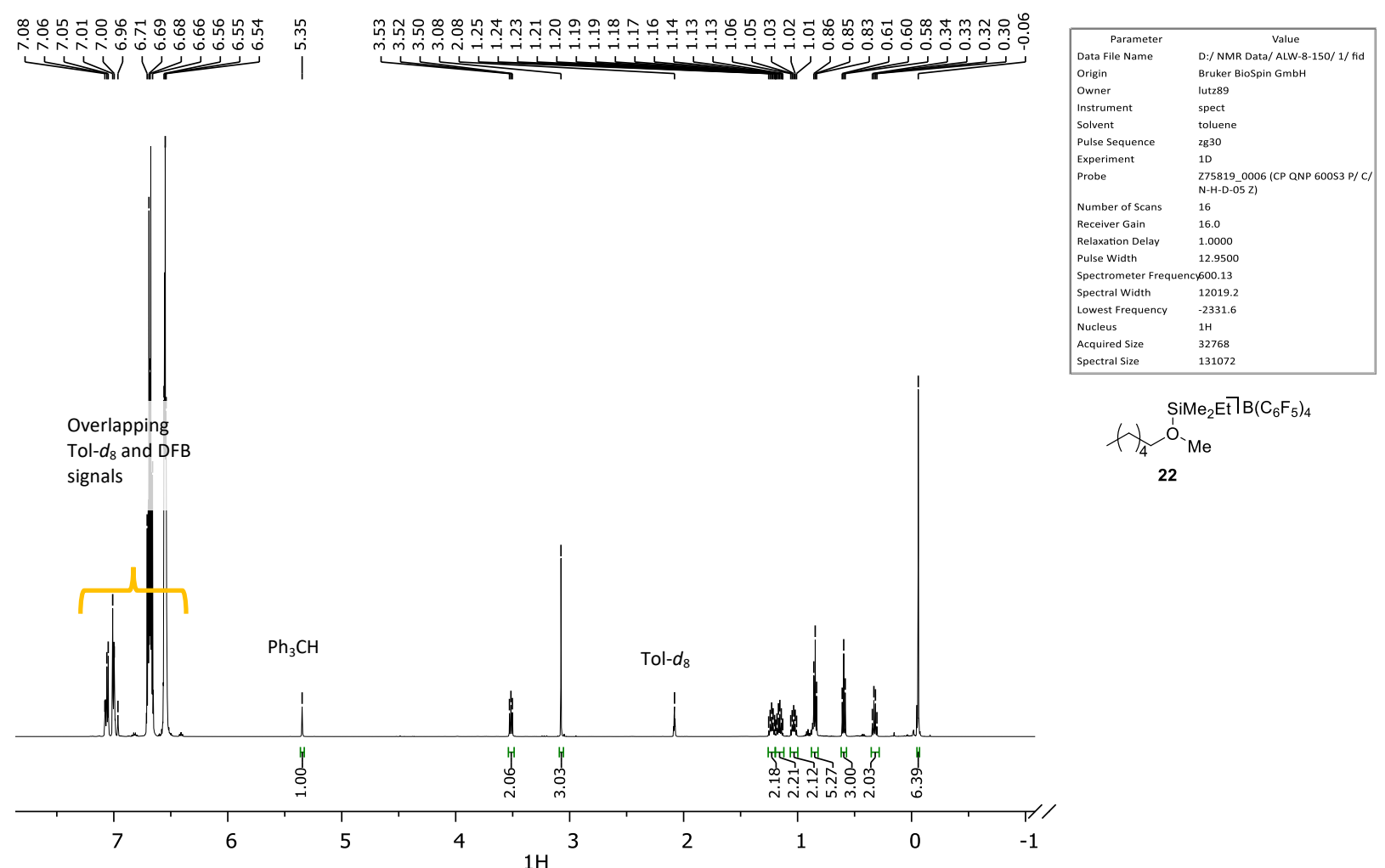

Figure S107: $600 \mathrm{MHz}{ }^{1} \mathrm{H}$ NMR spectrum of 22 at room temperature. Solvent: 1:5 DFB:Tol- $d_{8}$.

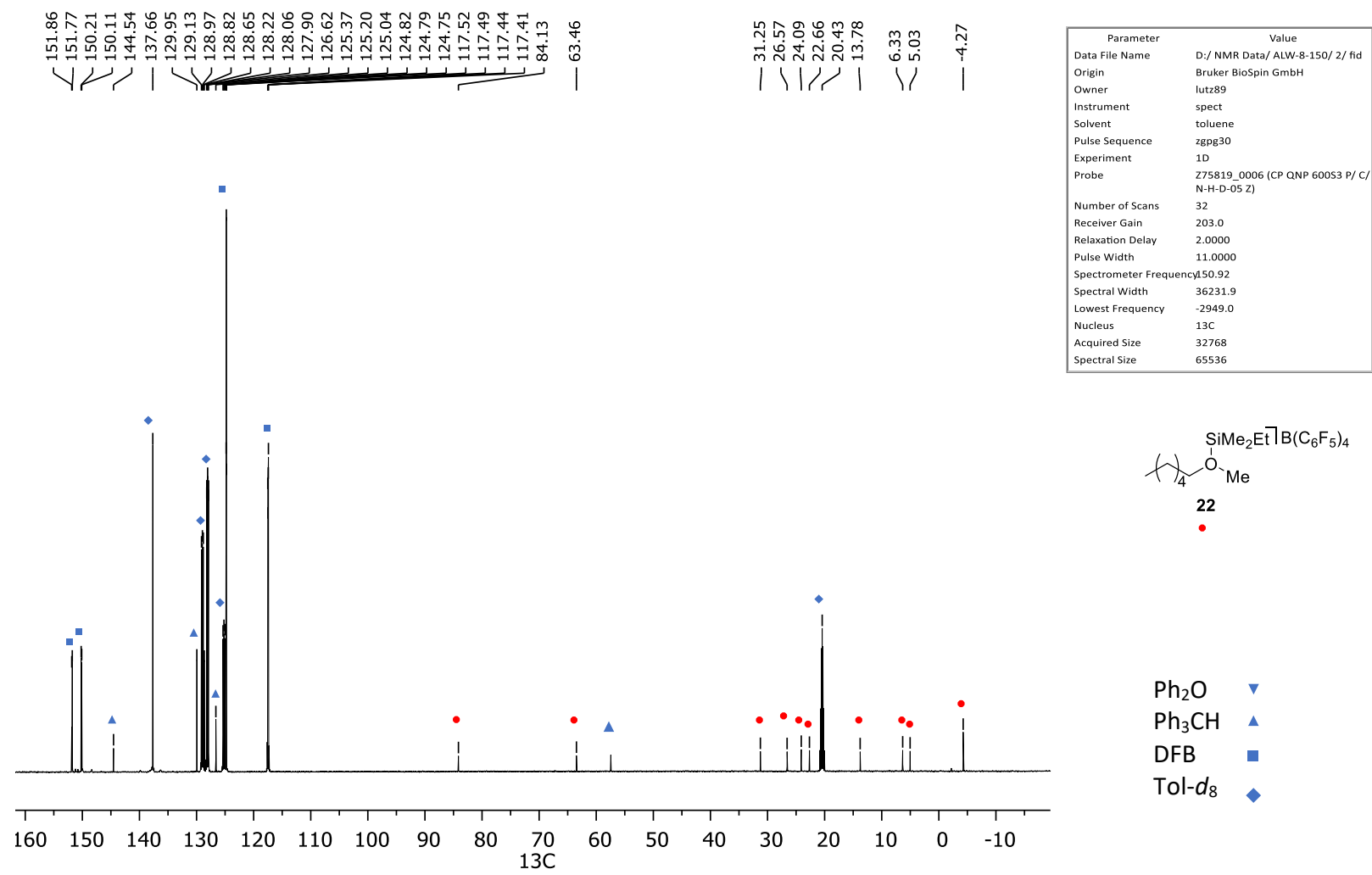

Figure S108: $151 \mathrm{MHz}{ }^{13} \mathrm{C}\left\{{ }^{1} \mathrm{H}\right\}$ NMR spectrum of 22 at room temperature. Solvent: 1:5 DFB:Tol$d_{8}$. 


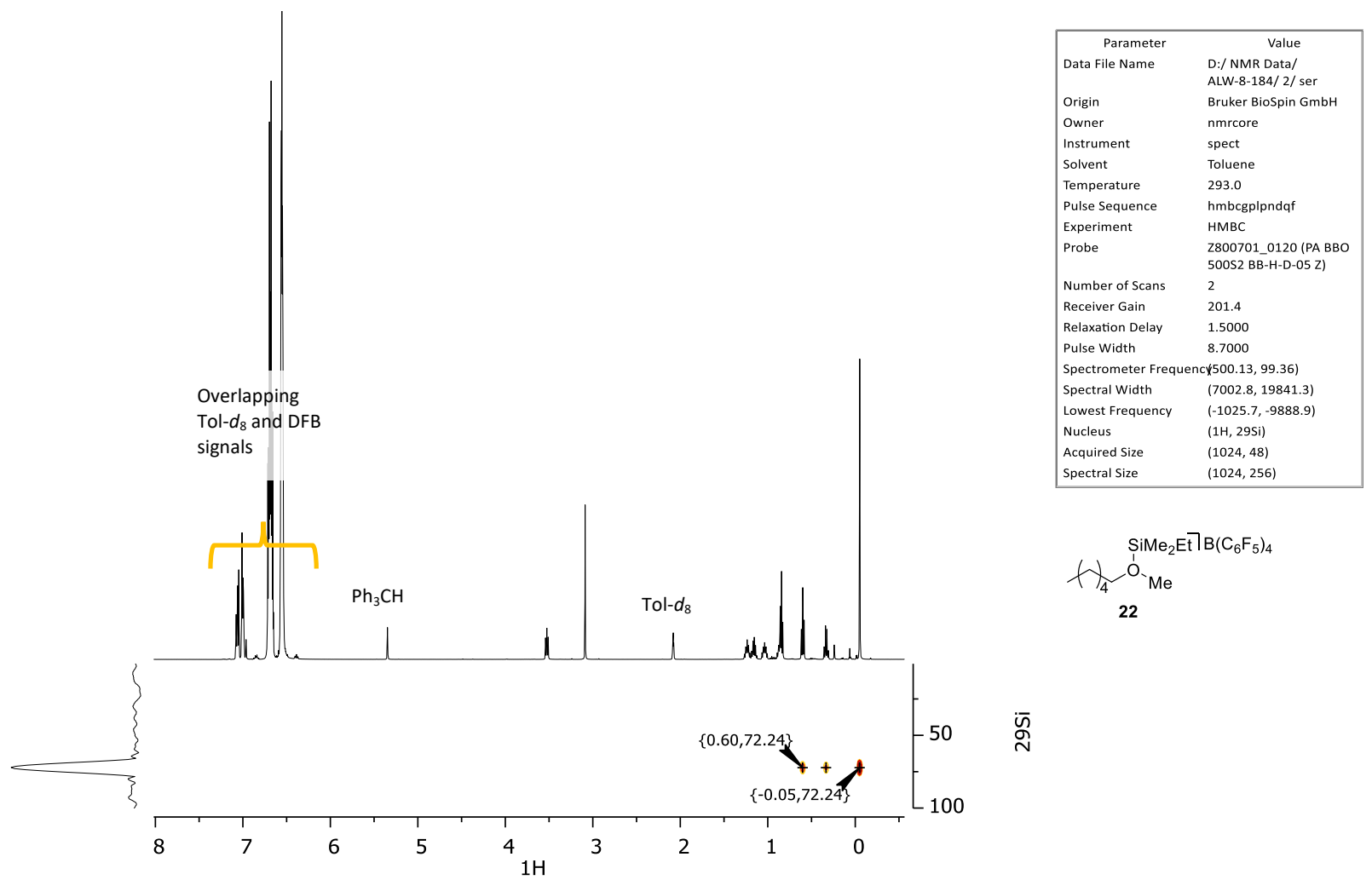

Figure S109: (500 MHz, $99 \mathrm{MHz}){ }^{1} \mathrm{H},{ }^{29} \mathrm{Si}-\mathrm{HMBC}$ NMR spectrum of 22 at room temperature. Solvent: 1:5 DFB:Tol- $d_{8}$. 
NMR Spectra for Intermediate 7.
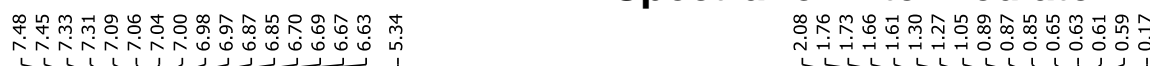

।
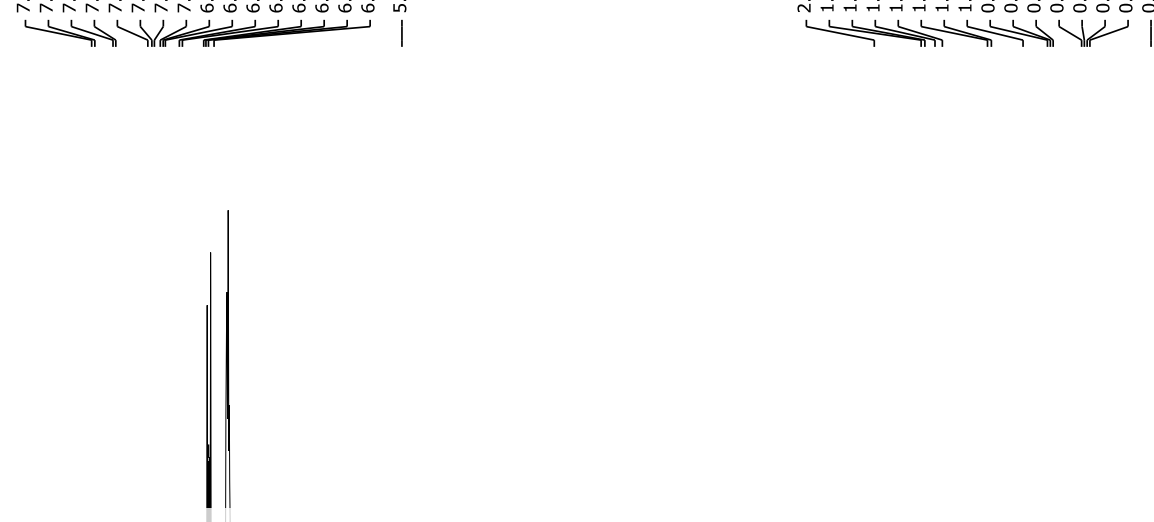

Overlapping Tol- $d_{8}$

$\mathrm{DFB}, \mathrm{Ph}_{2} \mathrm{O}, \mathrm{Ph}_{3} \mathrm{CH}$, and

7 signals
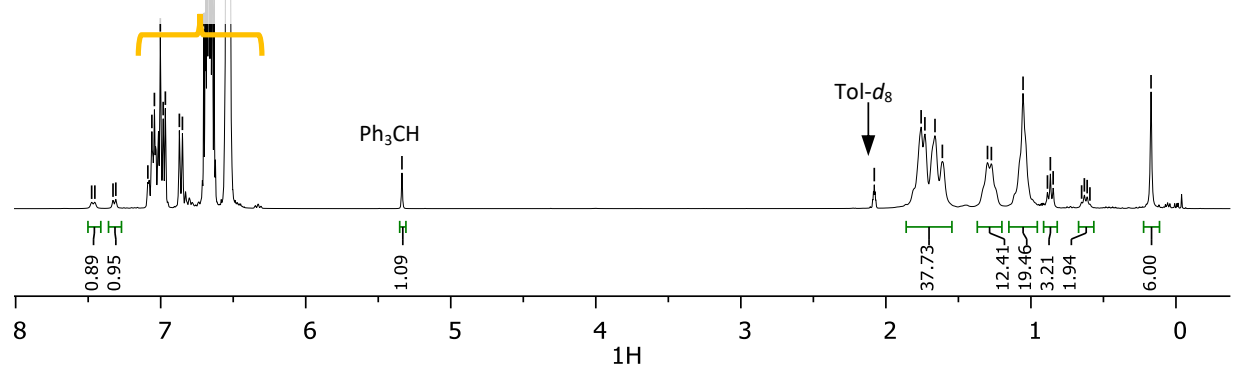

Figure S110: $400 \mathrm{MHz}{ }^{1} \mathrm{H}$ NMR spectrum of 7 at $0{ }^{\circ} \mathrm{C}$. Solvent: 1:5 DFB:Tol- $d_{8}$.

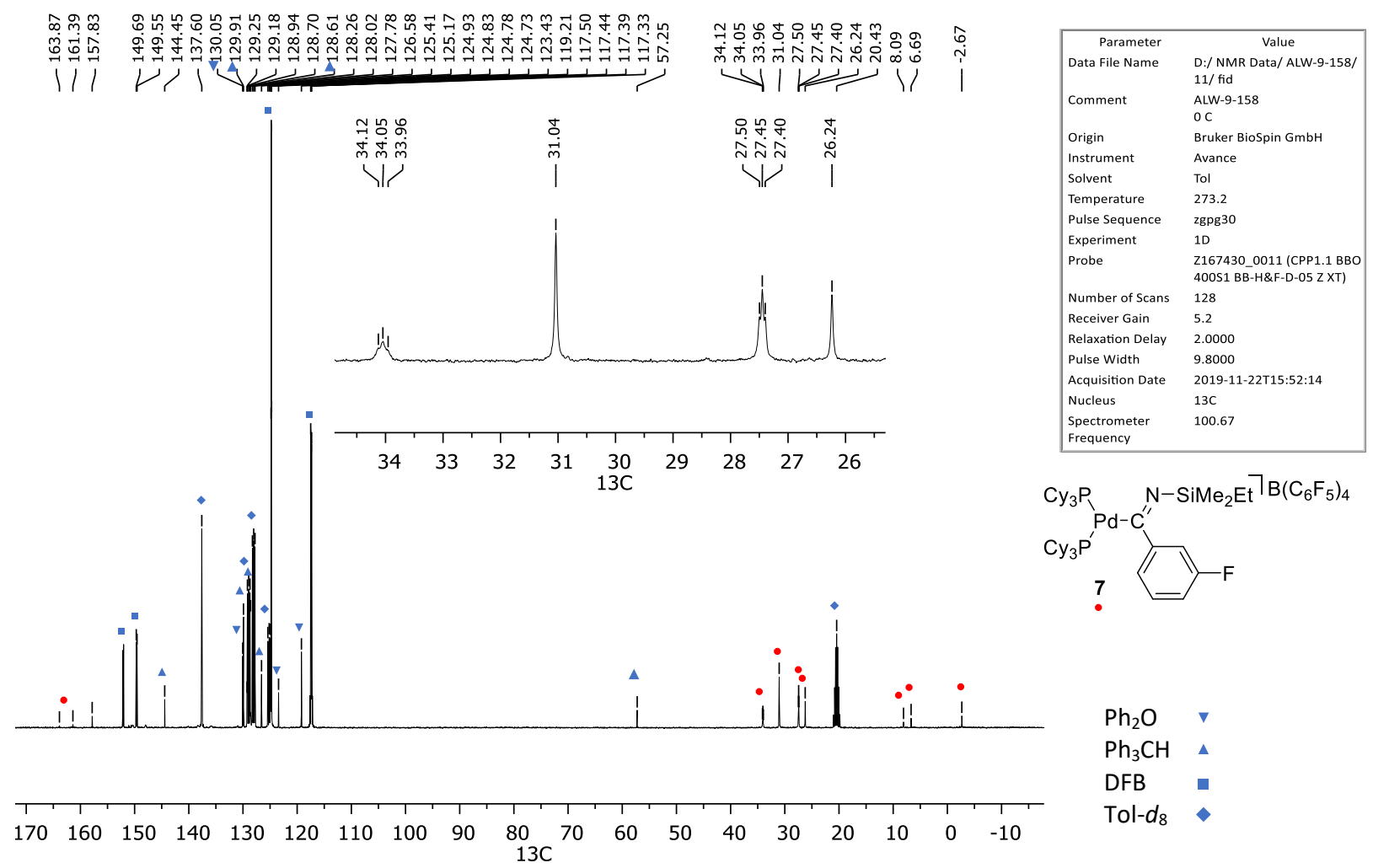

Figure S111: $101 \mathrm{MHz}{ }^{13} \mathrm{C}\left\{{ }^{1} \mathrm{H}\right\}$ NMR spectrum of 7 at $0{ }^{\circ} \mathrm{C}$. Solvent: 1:5 DFB:Tol- $d_{8}$. 


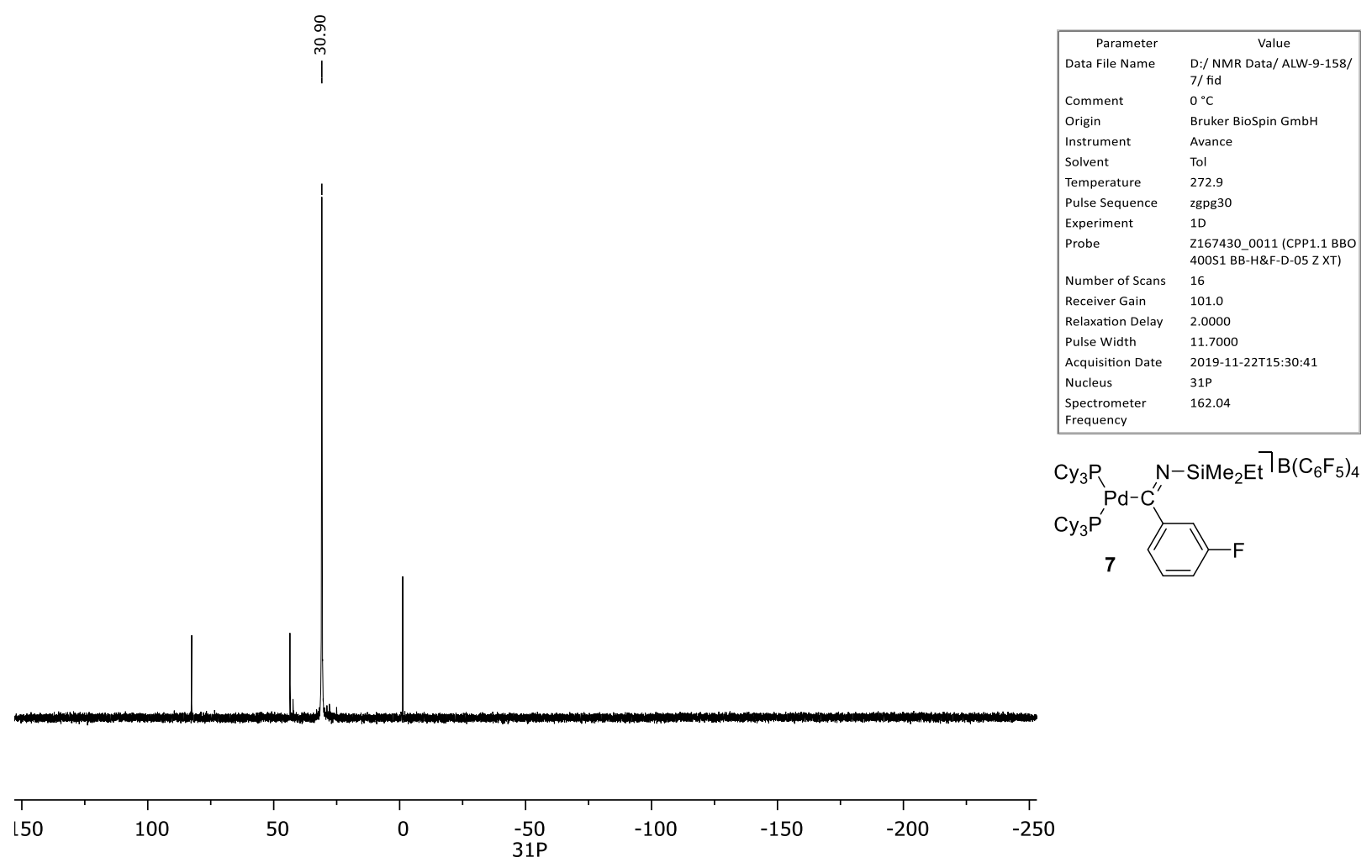

Figure S112: $162 \mathrm{MHz}{ }^{31} \mathrm{P}\left\{{ }^{1} \mathrm{H}\right\}$ NMR spectrum of 7 at $0{ }^{\circ} \mathrm{C}$. Solvent: 1:5 DFB:Tol- $d_{8}$.

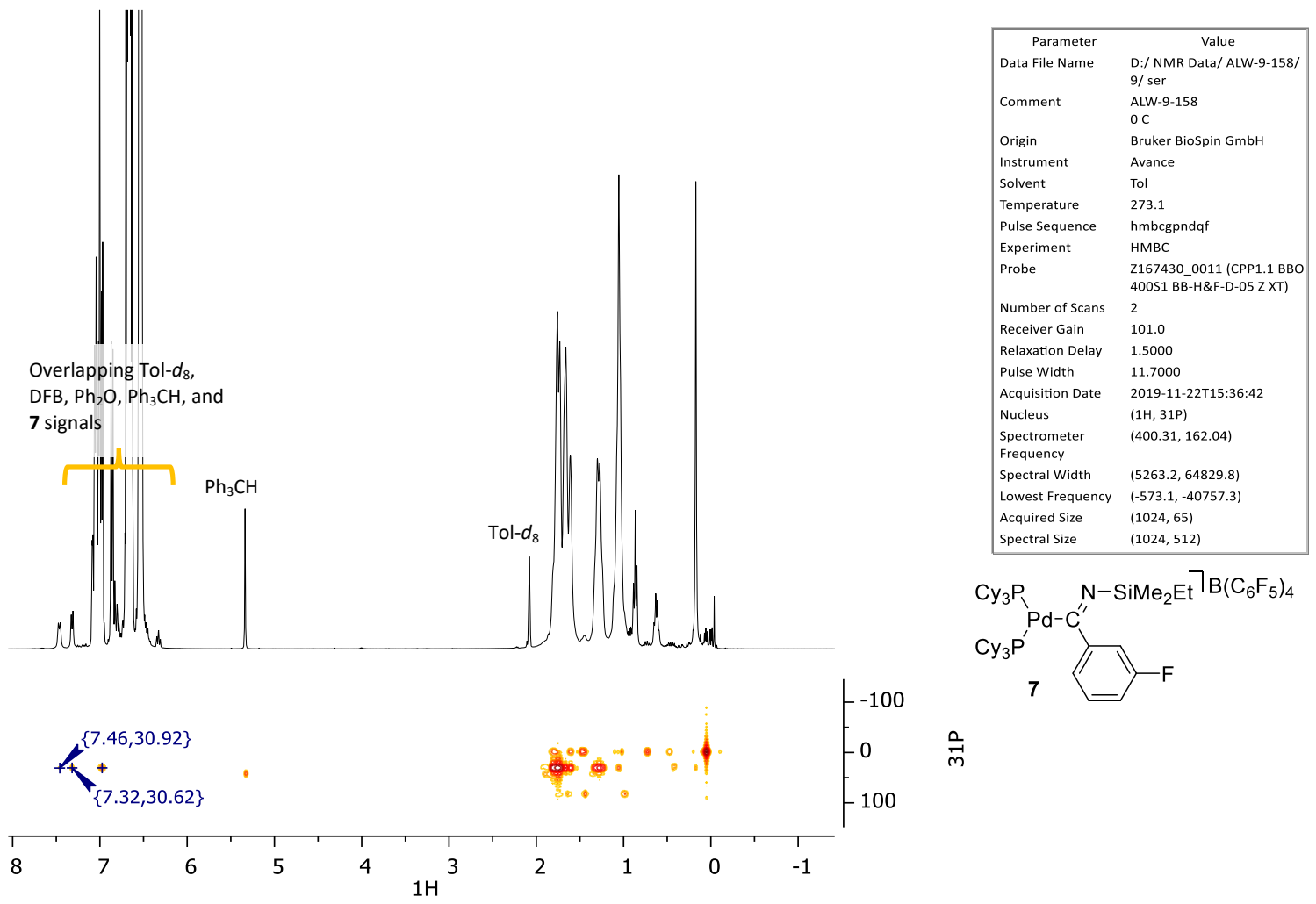

Figure S113: (400 MHz, $162 \mathrm{MHz}$ ) ${ }^{1} \mathrm{H},{ }^{31} \mathrm{P}-\mathrm{HMBC}$ NMR spectrum of 7 at $0{ }^{\circ} \mathrm{C}$. Solvent: $1: 5$ DFB:Tol-d $d_{8}$. 


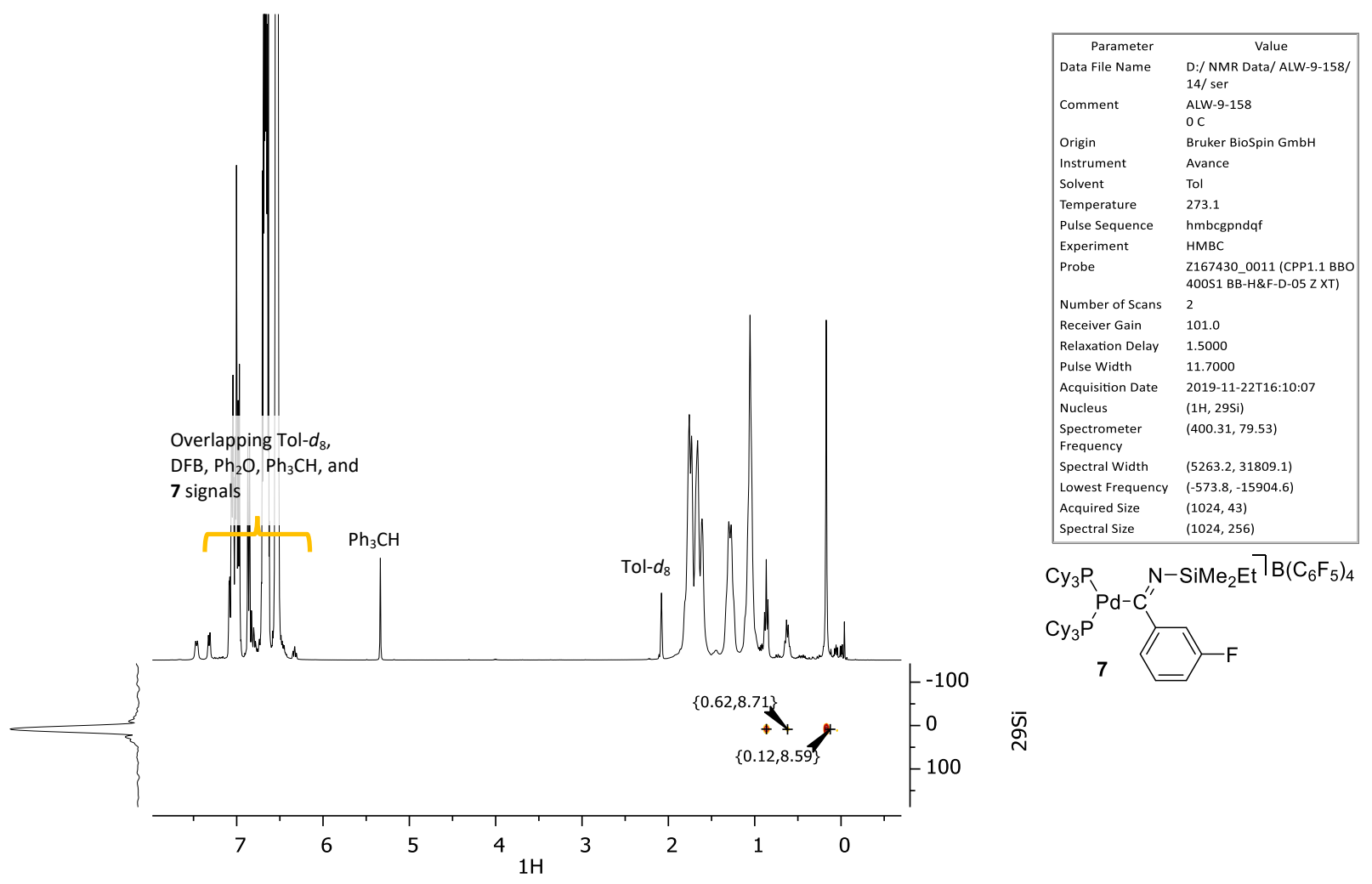

Figure S114: $(400 \mathrm{MHz}, 80 \mathrm{MHz}){ }^{1} \mathrm{H},{ }^{29} \mathrm{Si}-\mathrm{HMBC}$ NMR spectrum of 7 at $0{ }^{\circ} \mathrm{C}$. Solvent: 1:5 DFB:Tol$d_{8}$. 


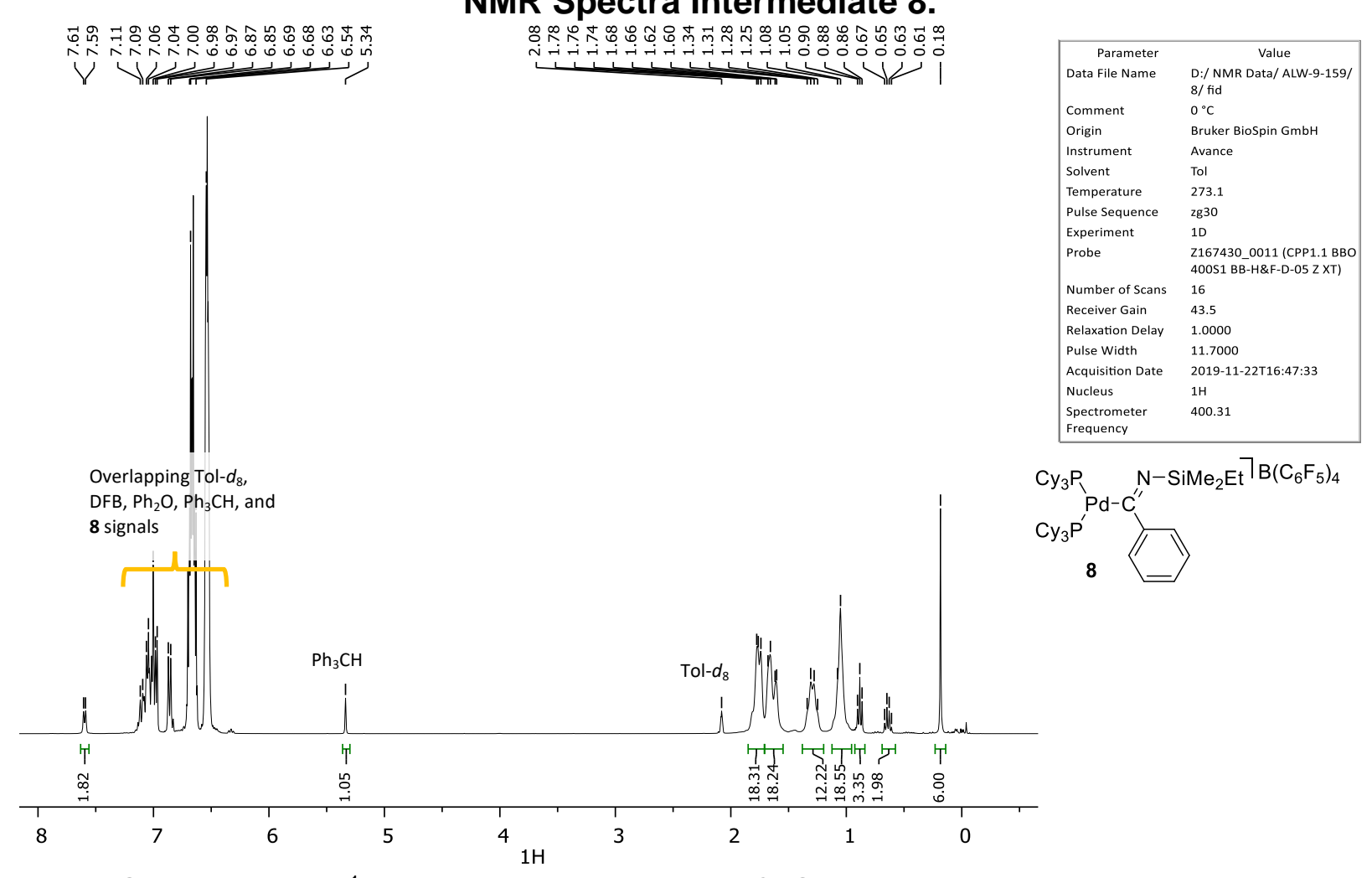

Figure S115: $400 \mathrm{MHz}{ }^{1} \mathrm{H}$ NMR spectrum of 8 at $0{ }^{\circ} \mathrm{C}$. Solvent: 1:5 DFB:Tol- $d_{8}$.

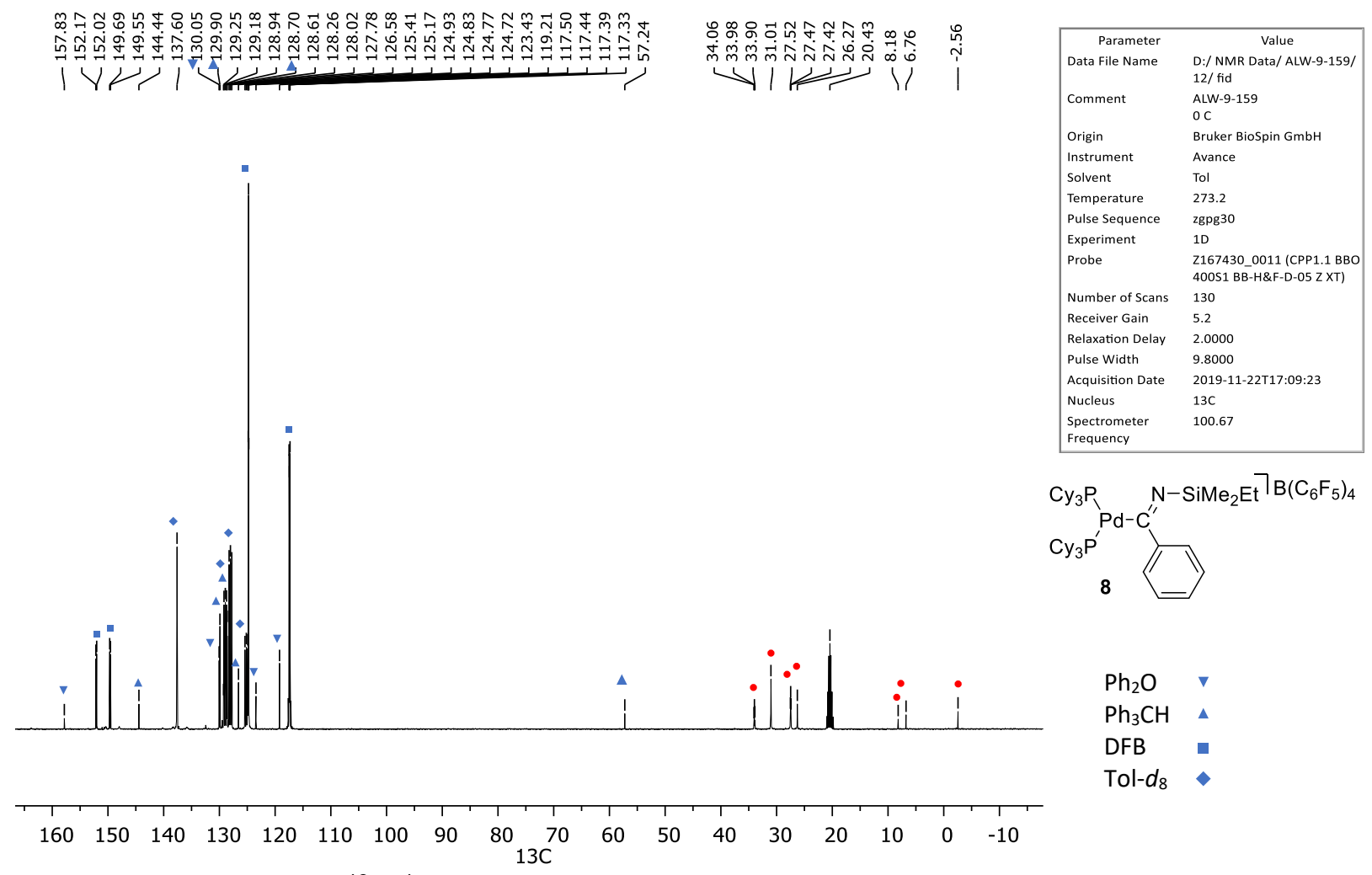

Figure S116: $101 \mathrm{MHz}{ }^{13} \mathrm{C}\left\{{ }^{1} \mathrm{H}\right\}$ NMR spectrum of 8 at $0{ }^{\circ} \mathrm{C}$. Solvent: 1:5 DFB:Tol- $d_{8}$. 


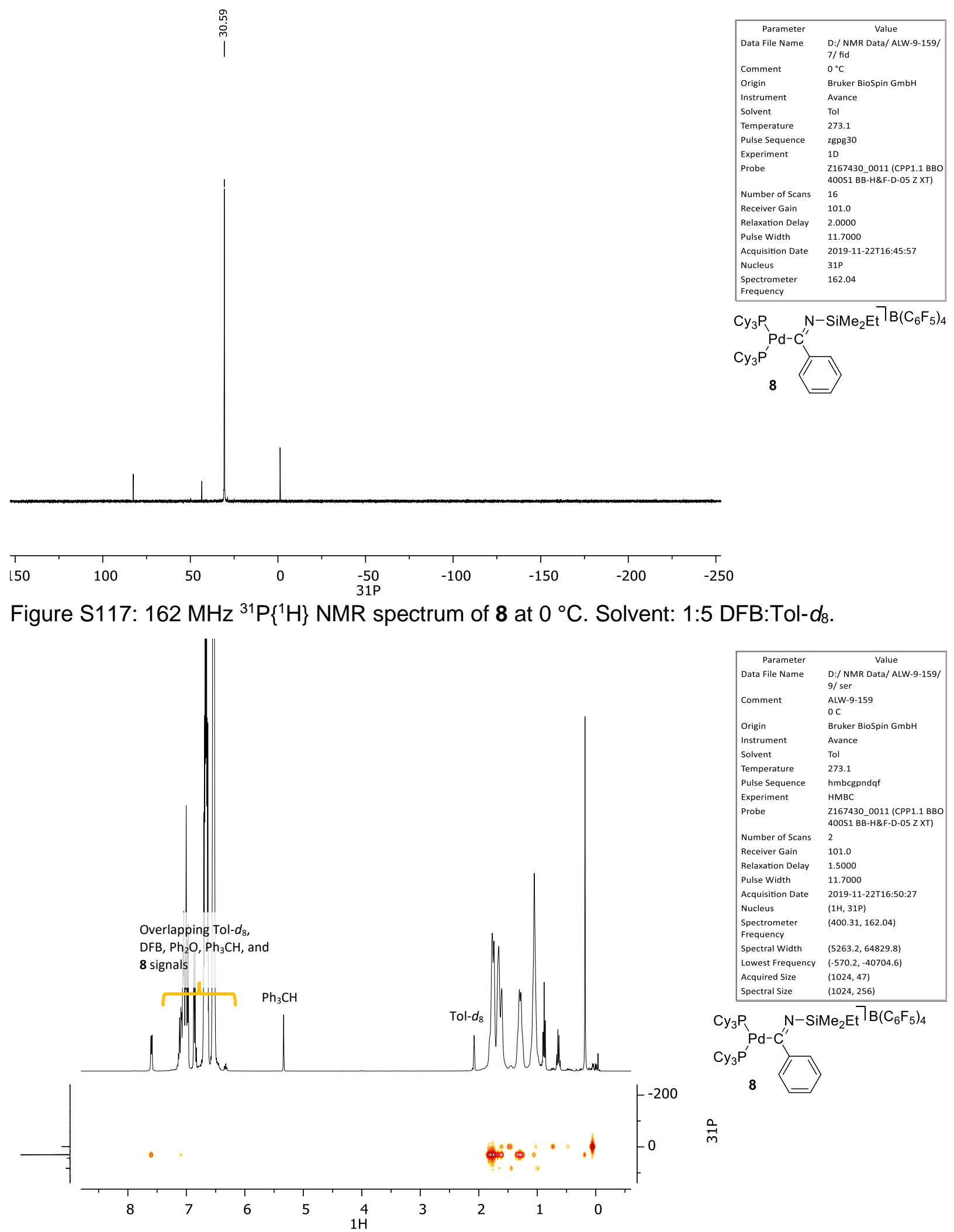

Figure S118: (400 MHz, $162 \mathrm{MHz}$ ) ${ }^{1} \mathrm{H},{ }^{31} \mathrm{P}-\mathrm{HMBC}$ NMR spectrum of 8 at $0{ }^{\circ} \mathrm{C}$. Solvent: $1: 5$ DFB:Tol- $d_{8}$. 


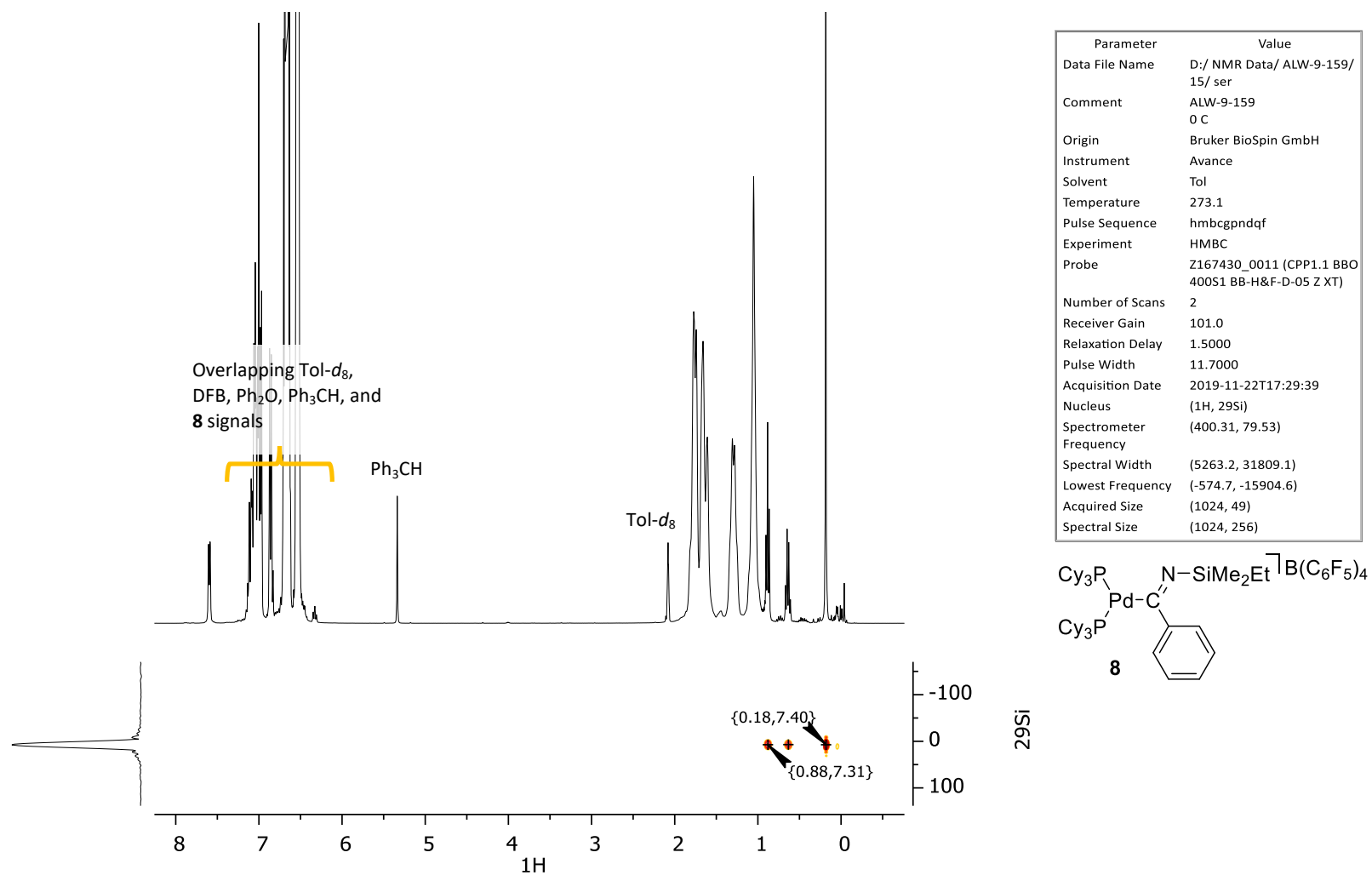

Figure S119: $(400 \mathrm{MHz}, 80 \mathrm{MHz}){ }^{1} \mathrm{H},{ }^{29} \mathrm{Si}-\mathrm{HMBC}$ NMR spectrum of 8 at $0{ }^{\circ} \mathrm{C}$. Solvent: 1:5 DFBTol-d8. 


\section{References}

(1) Tanabe, M.; Ishikawa, N.; Osakada, K. Preparation and Structure of a New Dipalladium Complex with Bridging Diphenylgermyl Ligands. Diverse Reactivities of $\mathrm{Pd}\left(\mathrm{PCy}_{3}\right)_{2}$ and $\mathrm{Pt}\left(\mathrm{PCy}_{3}\right)_{2}$ toward $\mathrm{Ph}_{2} \mathrm{GeH}_{2}$. Organometallics 2006, 25, 796-798.

(2) Wierschen, A. L.; Romano, N.; Lee, S. J.; Gagné, M. R. Silylpalladium Cations Enable the Oxidative Addition of $\mathrm{C}\left(\mathrm{sp}^{3}\right)-\mathrm{O}$ Bonds . J. Am. Chem. Soc. 2019, 141, 16024-16032.

(3) Omann, L.; Oestreich, M. Catalytic Access to Indole-Fused Benzosiloles by 2-Fold Electrophilic C-H Silylation with Dihydrosilanes. Organometallics 2017, 36, 767-776.

(4) Fulmer, G. R.; Miller, A. J. M.; Sherden, N. H.; Gottlieb, H. E.; Nudelman, A.; Stoltz, B. M.; Bercaw, J. E.; Goldberg, K. I. NMR Chemical Shifts of Trace Impurities: Common Laboratory Solvents, Organics, and Gases in Deuterated Solvents Relevant to the Organometallic Chemist. Organometallics 2010, 29, 2176-2179.

(5) Gaussian 09, Revision E.01, M. J. Frisch, G. W. Trucks, H. B. Schlegel, G. E. Scuseria, M. A. Robb, J. R. Cheeseman, G. Scalmani, V. Barone, G. A. Petersson, H. Nakatsuji, X. Li, M Caricato, A. Marenich, J. Bloino, B. G. Janeska, R. Gomperts, B. Mennucci, H. P. Hratchian, J. B. Ortiz, A. F. Izmaylov, J. L. Sonnenberg, D. Williams-Young, F. Ding, F. Lipparini, R. Egidi, J. Goings, B. Peng, A. Petrone, T. Henderson, D. Ranasinghe, B. G. Zakrzewski, J. Gao, N. Rega, G. Zheng, W. Liang, M. Hada, M. Ehara, K. Toyota, R. Fukuda, J. Hasegawa, M. Ishida, T. Nakajima, Y. Honda, O. Kitao, H. Nakai, T. Vreve, K. Throssel, J. A. Montgomery, Jr., J. E. Peralta, F. Ogliaro, M. Bearpark, J. J. Heyd, E. Brothers, K. N. Kudin, V. N. Staroverov, T. Keith, R. Kobayashi, J. Normand, K. Raghavachari, A. Rendell, J. C. Burant, S. S. Iyengar, J. Tomasi, M. Cossi, J. M. Millam, M. Klene, C. Adamo, R. Cammi, J. W.

Ochterski, R. L. Martin, K. Morokuma, O. Farka, J. B. Foresman, and D. J. Fox, Gaussian, Inc., Wallingford CT, 2016.

(6) Binh, D. H.; Milovanović, M.; Puertes-Mico, J.; Hamdaoui, M.; Zarić, S. D.; Djukic, J.$\mathrm{P}$. Is the $\mathrm{R}_{3} \mathrm{Si}$ Moiety in Metal--Silyl Complexes a Z Ligand? An Answer from the Interaction Energy. Chem.-Eur. J. 2017, 23, 17058-17069. 